Keywords: Plutonium Immobilization, Ceramic, Powder Processing

Retention Time: Permanent

\title{
SUMMARY OF GRANULATION MATRIX TESTING FOR THE PLUTONIUM IMMOBILIZATION PROGRAM (U)
}

September 21, 2001

C. C. Herman

Westinghouse Savannah River Company Savannah River Technology Center Aiken, SC 29808

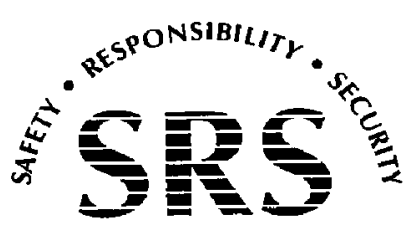

SAVANNAH RIVER SITE

PREPARED FOR THE U.S. DEPARTMENT OF ENERGY UNDER CONTRACT NO. DE-AC09-96SR18500 
This document was prepared in conjunction with work accomplished under Contract No. DE-AC09-96SR18500 with the U.S. Department of Energy.

\section{DISCLAIMER}

This report was prepared as an account of work sponsored by an agency of the United States Government. Neither the United States Government nor any agency thereof, nor any of their employees, makes any warranty, express or implied, or assumes any legal liability or responsibility for the accuracy, completeness, or usefulness of any information, apparatus, product or process disclosed, or represents that its use would not infringe privately owned rights. Reference herein to any specific commercial product, process or service by trade name, trademark, manufacturer, or otherwise does not necessarily constitute or imply its endorsement, recommendation, or favoring by the United States Government or any agency thereof. The views and opinions of authors expressed herein do not necessarily state or reflect those of the United States Government or any agency thereof.

This report has been reproduced directly from the best available copy.

Available for sale to the public, in paper, from: U.S. Department of Commerce, National Technical Information Service, 5285 Port Royal Road, Springfield, VA 22161

phone: (800) 553-6847

fax: (703) 605-6900

email: orders@ntis.fedworld.gov

online ordering: http://www.ntis.gov/support/index.html

Available electronically at http://www.doe.gov/bridge

Available for a processing fee to U.S. Department of Energy and its contractors, in paper, from: U.S. Department of Energy, Office of Scientific and Technical Information, P.O. Box 62, Oak Ridge, TN 37831-0062

phone: (865)576-8401

fax: $(\mathbf{8 6 5}) 576-5728$

email: reportseadonis.osti.gov 


\section{SUMMARY OF GRANUlation MATRIX TESTING FOR THE Plutonium Immobilization Program (U)}

September 21, 2001

\section{Document Approvals}

C. C. Herman, Immobilization Technology Section Westinghouse Savannah River Company Author

D. K. Pugh, Analytical and Nuclear Chemistry Division Lawrence Livermore National Laboratory

Technical Reviewer

D.T. Herman, Immobilization Technology Section Westinghouse Savannah River Company

Technical Reviewer

J.C. Marra, Waste Processing Technology Section

Westinghouse Savannah River Company

PIP Program Manager

E. W. Holtzscheiter, Immobilization Technology Section Section Manager

Authorized Derivative Classifier
Date

Date

Date 
WSRC-TR-2001-00454

Revision 0

\section{INTRODUCTION}

In FY00, a matrix for process development testing was created to identify those items related to the ceramic process that had not been fully developed or tested and to help identify variables that needed to be tested. This matrix, NMTP/IP-99-003, was jointly created between LLNL and SRTC and was issued to all affected individuals. The matrix was also used to gauge the progress of the development activities. As part of this matrix, several series of tests were identified for the granulation process. This summary provides the data and results from the granulation testing. The results of the granulation matrix testing were used to identify the baseline process for testing in the PuCTF with cold surrogates in B241 at LLNL.

\section{BACKGROUND/GRANULATION OBJECTIVES}

The granulation step in the plutonium immobilization process is being performed to agglomerate the fine particles of ceramic oxides and actinide oxides that have been milled and blended in the attritor. Through granulation, the fine particles are agglomerated into larger clusters to improve powder properties. Most important of these are improved flowability, decreased dustiness, and increased powder bulk density. These properties are important for ensuring that die filling is reproducible and for minimizing the amount of dust released into the glovebox since the powders are not contained during pressing. For the granulation matrix testing, these properties were considered in measuring the success of the test variable.

\section{POWDER PREPARATION}

LLNL procedure TIP-PD-02, "Fabricating Surrogate Plutonium Ceramic Pucks - Plant Prototypical Process", was used to fabricate the batches of material. Each batch of material fabricated was documented on a batch data sheet. The batches were fabricated in individual $1.5-\mathrm{kg}$ precursor lots and lots were combined as needed to produce the nominal feed batch. All precursors were wet ball milled, dried, size-reduced, and then calcined. If the granulation test required the addition of a binder to the precursors, it was added using wet ball milling. The powder was then again dried and size-reduced. The precursors were then blended in the attritor with the appropriate amount of cerium oxide or the appropriate amounts of cerium and uranium oxides. If the granulation test required the addition of the binder during mixing/blending, it was added to the attritor with the other feed materials. Acrawax $\mathrm{C}$ was used when a dispersant was necessary in the attritor.

In some cases, the granulation gave very poor results and the powder was re-used in another test to minimize material and labor costs. If water was the binder added during granulation, then the powder was dried and re-attritored to break up the particles. If an organic binder was added during granulation, then the powder was heated in the calcine oven to a minimum of $450^{\circ} \mathrm{C}$ to ensure that the binder had burned off and the powder was re-attritored to break up the particles. When these powders were used and gave spurious results, testing was repeated as necessary with fresh powder to verify results since a slight difference in powder behavior is seen because of the smaller particle sizes and slight binder residue. 
WSRC-TR-2001-00454

Revision 0

\section{GRANULATION TESTING}

Granulation matrix testing was performed using the baseline production process with cerium oxide as a surrogate for the plutonium and uranium oxides. Verification testing was also performed with uranium to confirm and further optimize the processing parameters. The $10.5 \mathrm{wt} \%$ plutonium composition was used in the testing since the studies were initiated before the formulation was officially changed. The matrix given in document NMTP/IP-99-003 was used to provide the guidance for the parameters and variables to be tested.

Most of the granulation testing was performed in the research and development labs in B241 at LLNL using the laboratory-scale 8-quart GEMCO granulator. However, testing that was performed to determine the optimal batch sizing used the larger PuCTF granulator. Copies of the granulation data sheets will be stored in a three ring binder labeled "Granulation Matrix Data Sheets", which will be archived with other data from the program.

\section{Parameters Tested}

Parameters investigated for this study included the following:

- Binder type, amount, and addition point;

- Maximum capacity of the granulator;

- Use of the Agitator;

- Lubricant type and amount; and

- Necessity for drying.

As these parameters were tested, they were verified with the nominal batch size and with powders containing uranium oxide. Uranium containing powders had previously been shown to behave differently during granulation; thus, verification of the parameters was considered essential. Originally, it was planned to use manganese oxide for some of the verification testing, but due to the disposal problems of this material, the use of uranium oxide was considered adequate.

Other parameters that were investigated during the study but that were not completely resolved included the following:

- Type and size of nozzle;

- Nozzle position;

- Rate of binder addition;

- Vessel speed;

- Process time; and

- Powder temperature.

Since these parameters were felt to be equipment size dependent, they could not be finalized until testing with full-scale equipment was performed. The studies documented here give a synopsis of the optimum parameters for the lab-scale equipment, which should serve as the starting point for the development of the parameters for the full-size equipment. 
Appendix A is an excerpt from the overall process development matrix, but contains a status of the granulation matrix parameters only. This matrix provides a concise summary of the studies performed.

Binder Type, Amount, and Addition Point

A total of seven different binders were selected for the granulation matrix testing. The binder type was selected based on the results from Test \#9 and \#10 of the Form \& Process Development Task Plan (PIP-TP-98-001), which was performed at LLNL. Although seven different binders were selected, the number of tests was much larger due to the additional variables of the amount of binder (both binder concentration and actual addition amount), addition point for the binder, and the combination of binders. Forty-five different variables were tested with respect to the binder parameter. Appendix B gives a summary of the combinations tested, along with the precursor/powder batch ID.

In these original binder tests, the same nozzle size and pattern was used throughout; however, spraying water used a different size nozzle than organic binder. The agitator arm was not used and process time was kept constant at 30 minutes.

As mentioned earlier, uranium oxide was used for verification testing. Binder combinations/concentrations that seemed promising were tested with uranium oxide, and Appendix $\mathrm{C}$ identifies those binders and precursor/powder batches that were tested with uranium.

\section{Maximum Capacity}

The larger-scale PuCTF granulator was used to perform a series of test to determine the maximum capacity of the granulator. The PuCTF granulator is nominally rated for $10-\mathrm{kg}$ (or 10 liters) processing but will accommodate much larger quantities. Batch size was increased in 2-kg increments up to 18-kg to determine an optimal and maximum batch size from a processing perspective. The same performance measures investigated for the binder testing were used to ascertain the quality of the product produced.

\section{Use of the Agitator}

The GEMCO tumble granulator selected for lab-scale and PuCTF testing came equipped with an agitator bar to help with mixing of the powder being processed. Preliminary studies showed that use of the agitator during binder addition helped to disperse the binder. However, the use of the agitator presented additional maintenance issues because of the extra seal required and additional support equipment. Therefore, one of the parameters examined was whether the agitator was beneficial to processing. The agitator was only investigated for use during binder addition. The same performance measures investigated for the binder testing were used to ascertain the quality of the product produced. It was determined that the agitator gave no significant advantages to powder processing during granulation and its use was discontinued. 
WSRC-TR-2001-00454

Revision 0

Lubricant Type and Amount

When the process development matrix was created, it was not certain whether a lubricant would be necessary during pressing to avoid creating undue stress on the pucks. Lab-scale studies to date had shown that some of the binders would require the addition of a lubricant, while others would not and nothing was known about the effect in the fullyautomatic press. With each binder system tested, pressing was evaluated to determine if lubricant would be necessary. However, no extensive testing with lubricants was performed because the more optimal binder systems did not require any additional lubricant to be added. Should a lubricant prove to be necessary in the future, the results from Test \#15 of the Form \& Process Development Task Plan (PIP-TP-98-001) showed that a small amount $(<1 \mathrm{wt} \%$ total) of Acrawax $\mathrm{C}$ added to the powder would greatly reduce the stresses created during ejection of the puck from the press.

\section{Necessity for Drying}

An additional option for the GEMCO granulator is a heated shell to dry the product. In this configuration, the GEMCO is called a "Formulator" and product can be heated to drive off any excess moisture. This equipment was tested at the vendor and in early development studies. However, as testing with the normal configuration progressed, the need for drying the granulated product was eliminated because of the acceptable product obtained.

\section{Other Parameters}

Most of the testing performed for this matrix used a nozzle with a fan spray pattern with two different size openings. The size selected gave the lowest hold-up and best granulated product for the nozzles tested. Some preliminary testing was performed with the use of a cone-shaped nozzle, but problems with over-spray were seen with binder solutions. However, the limited testing did indicate that water spray with the cone-shaped nozzle was effective. The nozzle type and size is one of the parameters that will be spray arm location dependent and also very dependent on the binder solution; therefore, the final selection should be performed with equipment that is representative of the plant-size, process, and configuration.

As alluded to in the previous paragraph, the nozzle position will also strongly impact the hold-up and resulting granulated product. For the granulation matrix testing, very little work was performed with changing the nozzle position. For the lab-scale granulator (i.e., nominal $8 \mathrm{qt}$ working volume), the nozzle arm was slightly off of center such that the powder was sprayed before the tumbling process was initiated. The nozzle position is another parameter that should be tested in the plant-prototypical equipment.

The rate of binder addition was kept fairly constant within binder concentrations tested, but was varied with the different binder types and amounts to try to optimize the spray pattern. It was mainly controlled through the setting of the pump, however, in plant production, this should be controlled using a metered rate. Rates of binder addition are 
given for the different tests. However, this is another parameter that will have to be optimized with the plant-prototypical equipment.

The initial vessel speed was kept at a maximum from test to test and during binder addition. The speed of tumbling was varied with different binders because of the flow characteristics of the powder depending on the binder. The vessel speeds for the lab-scale test unit were used to calculate the vessel speeds for the PuCTF unit and should also be used as a starting reference for the plant-prototypical equipment.

Initial process time was 30 minutes for all testing. However, after a significant portion of the testing was performed, it was determined that shorter process times would result in less hold-up in the vessel and less degradation of the soft granules. Using the acrylic lid, it was observed that the powder reached an optimal characteristic and then the material would start clinging to the sides of the vessel with additional tumbling time. Therefore, the process time was reduced such that the process was ended after the powder appeared to be fully granulated and before the material started accumulating on the sides of the vessel. For the lab-scale unit, this was about 10 minutes of total process time, while the PuCTF unit required about 20 minutes of process time. This of course will have to be optimized with the plant-scale unit.

Powder from the attritor has an elevated temperature due to the high-energy exerted on the powder during milling/blending. Some of the tests used powder that was directly transferred from the attritor into the granulator. With some of the binders, this resulted in excessive hold-up in the vessel due to steam generation in the vessel. For the matrix testing, the powder used was at room temperature to eliminate this variable. Limited testing in the PuCTF granulator was performed to try to determine the maximum processing temperature. However, these studies were not completed and additional testing will have to be performed in the plant-prototypical equipment to determine the upper temperature limit for powder processing.

\section{Granulated Powder Testing}

Powders produced were compared based on the flow and dust characteristics, the particle size distribution (including general product appearance), the puck quality, and Powder Test Center data. The methods used for the comparison are described below, along with discussion of the results. Although not directly related to powder properties, the amount of hold-up left in the vessel was also a critical parameter evaluated for each test. Some discussion on this also follows.

Summaries of the powder and puck testing results and general observations about the granulation tests performed with cerium are given as Appendix D and with uranium are given as Appendix E. The "Matrix Test \#" refers to the test number and the amount of binder added during the granulation process. A description of the binder type, amount, and addition point being tested in the individual tests was given in Appendix B and this is what was referred to as the test number. An example of a "Matrix Test \#" would be "GA-4- 
10\%." The "GA-4" refers to a test in which 6\% Airvol 21-205 is added to the powder before mixing and the " $-10 \%$ " refers to 10 weight percent of water that was added during granulation.

Flow

One of the key reasons for performing the granulation step is to produce a powder that more evenly flows into and fills the press die cavity. The general flow characteristics of the granulated powders were evaluated by transferring some of the granulated product into a 1 " opening stainless steel funnel and determining how the material flowed. Each product was rated on its general flow behavior through the funnel. The test was repeated by packing the powder into the funnel and then observing the flow behavior for a second time. Observations are recorded in Appendix D and E. Powders were rated from "poor" to "very good" with respect to flow. Binder combinations that produced powders with less than "ok" flow were eliminated from further consideration.

In general, the binders had an optimal addition amount during granulation with too low or too high additions producing non-flowable powder. A nominal addition range was 10 to 12 weight percent. PEG and HPMC, especially when used together, tended to produce powders with good flow. Certain combinations or addition methods of Airvol and Duramax also produced good flowing powder.

In addition to this visual test, a larger-scale flow-meter was also constructed so the angle of repose for the granulated powders could be measured. This device used approximately 500-1000 $\mathrm{g}$ of powder and then the slide gate was removed and the resulting angle was measured using a protractor. The test was performed a minimum of three times with each powder and then the average was reported. This test was not performed for all of the powders because the apparatus was not ready for testing at the start of the study. The results from this testing are also reported in Appendix D. Unfortunately, no general trends could be gleaned from the data.

For the maximum capacity testing, flow was an important decision factor. Many problems were seen when emptying the granulator if the powder amount was much greater than the nominal capacity. Therefore, $10 \mathrm{~kg}$ was considered to be the nominal capacity from a flow perspective.

Dust

Another strong objective of performing granulation is to reduce the dust that is generated during the pressing process since this is the primary point in the ceramic fabrication process where the powders are not contained. A quantitative measurement of the dust associated with each powder was not performed. The amount of small particles $(<150 \mu \mathrm{m})$ is also reported in the Particle Size Distribution subsection. A general comment on the overall dusting behavior of the powders from a strictly visual observation is reported in Appendix D and E. Binder combinations that resulted in powders that were "dusty" or "very dusty" were not considered for further testing. 
WSRC-TR-2001-00454

Revision 0

General observations about dusting included the following: dust was minimal with binder additions above 10 weight percent during granulation; sprayed binders tended to reduce dusting more than water additions; PEG/HPMC combined solutions required higher concentrations to reduce dusting; and uranium powders tended to have less dust than the equivalently processed cerium powders.

\section{Particle Size Distribution}

Approximately 500 grams of each powder was characterized using sieve analyses to determine the particle size of the product ranging from $75 \mu \mathrm{m}$ to $1 \mathrm{~mm}$, as well as the associated level of fines (i.e., material less than $106 \mu \mathrm{m}$ ). The general size of each powder is reported in Appendix D. In general, this data was used to determine the amount of fines associated with each product and to obtain a general idea of the packing efficiency associated with the particles. Binder combinations that produced fines greater than $20 \%$ were not considered for further testing.

Observations about the particle size included: spraying water produced small particles $(150 \mu \mathrm{m})$ except at high water addition levels on powders containing high levels of binder; spraying PEG solutions on the powders tended to produce a lot of fines except at high concentrations or when the solution was combined with HPMC; spraying Duramax solutions tended to greatly reduce the amount of fines; and spraying PEG or Airvol solutions on powders containing Airvol resulted in large sized particles being formed. In general, particle sizes were in the range of 75 to $300 \mu \mathrm{m}$.

This was also an important criteria for determining the maximum capacity. When the granulator was over-filled, the binder did not seem to get evenly distributed on the powder. Very large granules formed in the higher powder loading tests in the path of the binder spray.

Puck Quality

After granulation, a small amount of powder from each batch was pressed using the laboratory Carver press to obtain a general idea of green puck quality. The remaining powder was archived for pressing using the PuCTF Best press. After pressing, the pucks were sintered at $1350^{\circ} \mathrm{C}$ for 4 hours using a programmed heat-up and cool-down. The temperature used for the binder burn-out hold depended on the particular binder type being used. Pressing information and puck measurements were recorded on puck data sheets per the requirements of TIP-PD-02. A summary of the puck measurement data is given as Appendix F and density data is plotted in Figure 1a and Figure $1 \mathrm{~b}$ of this appendix. The puck data sheets from the pucks pressed in the Carver press will be stored in the three ring binder labeled "Granulation Matrix Data Sheets", while the puck data sheets from the PuCTF Best press will be archived in the "Best Press" puck data sheet binder. Both of these binders are being archived with other data from the program.

General observations about the quality of the pucks produced from the powders and puck densities are given in Appendix D and E. Theoretical densities were assumed to be 5.20 
$\mathrm{g} / \mathrm{cc}$ for hafnium/cerium/cerium pucks and $5.71 \mathrm{~g} / \mathrm{cc}$ for hafnium/cerium/uranium pucks. For the PuCTF pressed pucks, an average of the densities from all pucks pressed is reported. Small cracking that was attributed to gradients introduced by problems with the press dies were considered acceptable whereas significant cracking associated with binder burn-out or pressing in general were not. The powders were also evaluated for the need for lubrication during the pressing operation with those not requiring lubrication considered more favorable. For puck density, the target density was greater than $92 \%$ of theoretical but densities of greater than $90 \%$ were considered acceptable.

Observations about puck quality and pressing behavior included the following:

- Less pressure was required in the Best Press than in the laboratory Carver press. For equivalent pressures in the Best Press, a lot of lamination cracking in the puck was seen. The Best Press pucks also tended to have higher densities, which can be attributed to the dual-action pressing that should have resulted in fewer density gradients in the green puck.

- Die filling problems were seen when the binder amounts were too high (powder too moist).

- Airvol powder/crystals added to the attritor resulted in puck sintering problems.

- Adding PEG to the powders before granulation or spraying PEG solutions to nonbinder containing powders during granulation resulted in pucks with lamination cracks and poorly lubricated powders when pressed.

- Spraying combinations of PEG and HPMC also had inconsistent results with regards to sintered puck quality and need for lubrication, but, when 8\% HPMC solution was combined with $20 \%$ PEG solutions, the pucks were generally of good quality.

- Duramax, in general, produced good pucks but some problems were seen with cracking when pressure was too great and density problems were seen when the pressure was too low.

- Some radial cracking was seen in the pucks containing Airvol, which was attributed to the alignment problem with the dies.

- Airvol and PEG combinations produced good pucks but needed additional lubrication to minimize ejection pressure.

- When a large amount of granules $>1 \mathrm{~mm}$ were present, they tended to remain intact after pressing and sintering but had no apparent affect on density.

- With uranium, combinations of Airvol in the powder with Airvol sprayed during granulation produced the best quality pucks.

No examination of the pucks was performed using microscopy, since all of the binders and the granulation methods had previously been shown to have no affect on product mineralogy.

\section{Powder Test Center Data}

Samples of the powders were subjected to Powder Test Center (PTC) testing to help determine the physical properties of the powders. A summary of the data is given as Appendix G. Important graphs associated with the data are given as figures in the 
appendix. Due to the suspension of the program, testing on all of the powders was not completed. Therefore, only the results of the powders tested are provided.

Both the bulk and tap densities were measured on the powders and are plotted in Figure 1 of Appendix G. The bulk density is also referred to as the apparent density. It ranged from 1 to $1.7 \mathrm{~g} / \mathrm{cc}$ for the powders measured. In general, powders with higher apparent densities are preferred because they are less difficult to press. The tap density is the density of a loose powder subjected to a number of prescribed tappings. The tap density represents the highest packing density possible for a powder without the application of pressure. The tap density provides useful information on the packing of the loose powder during transportation and when it is in a container on a press before die filling. It varied from 1.1 to $1.9 \mathrm{~g} / \mathrm{cc}$. The tap density to bulk density ratio was also calculated for each sample. The ratio of the tap density to the bulk density gives a signature characteristic of the interparticle friction, and is called the Hausner ratio, useful in categorizing relative interparticle friction. For a spherical powder, the tap to bulk ratio will be slightly above unity. More irregular particle shapes undergo a larger density increase with vibration, resulting in a higher Hausner ratio. In general, more irregular particle shapes give lower packing densities. The ratios are plotted in Figure 2 of Appendix G. In general, the granulated powders produced using Duramax 1023 in the blended powder and adding water during granulation had the highest densities, along with spraying 5 or $10 \%$ Airvol solutions during granulation. Hausner ratios varied from 1.04 to 1.19. No general trends for the particular binders or in percent additions were seen.

Information on flow as visually quantified and as measured with the fabricated flow apparatus was reported above. The angle of repose was also measured as part of the standard suite of tests with the Powder Test Center. The angle of repose is accepted in the powder processing industry as the standard measure of powder flowability. It represents the ability of the powder to uniformly fill a die cavity, with lower angles representing more flowable powder. Typically, an angle of $30^{\circ}$ is a good measure of powder flowability. The results are plotted in Figure 3 of Appendix G. Duramax 1023 in the powder or spraying Airvol during granulation produced the most flowable powders. This was consistent with visual observations discussed earlier.

The slide coefficient is a measure of the frictional interactions between powder particles and die walls during compaction. For many materials, frictional effects at the die walls dominate during compaction. In some cases, the friction is the leading problem in dry pressing, resulting in poor or non-uniform green density, die sticking, and puck lamination and end capping. For a given powder and a given die material, the slide coefficient varies slightly with green density. The magnitude extends from 0 (infinite friction) to 1 (no friction) and relates directly the friction forces to the pressing forces. A value around 0.7 is considered moderate, with higher values indicating good or very good compaction properties and lower values suggesting a difficult compaction process. Difficult compaction results in relatively large frictional forces, which can lead to large density variations and, ultimately, large shrinkage variations. A plot of the slide coefficients for the 
various powders compacted at 2 and $5 \mathrm{ksi}$ is given as Figure 4 in Appendix G. None of the powders were above the target value of 0.7. Generally, the results indicated that spraying Airvol during granulation or water on Duramax 1023 containing powders had the highest slide coefficients. Higher green densities represented by higher compaction pressures increased the coefficient as expected but still were below the target.

The compactability coefficient is a material constant characterizing the ability of a powder to densify and represents interactions between powder particles during compaction. Powders with high coefficients are compacted to high densities with relatively little pressure. Compactability is also referred to as compressability. It can be defined for fullsize pucks by the compression ratio, which is the volume of the loose powder compared to the volume of the compacted powder or the green puck density compared to the apparent density. A plot of the compactability coefficients for compacts pressed at 2 and $5 \mathrm{ksi}$ using the PTC is given as Figure 5 in Appendix G. Trends were consistent with the slide coefficient data. In general, there was very little scatter in the data and the results were relatively the same.

The ejection pressure was measured on the small compacts made with the PTC. The ejection pressure reported is the compact ejection pressure averaged over the first $2.54 \mathrm{~mm}$ of compact travel in the PTC die after the starting pressure overshoot needed to initiate the movement of the compact. The data is plotted in Figure 6 of Appendix $G$ for compaction pressures of 2 and $5 \mathrm{ksi}$. In general, powders containing Duramax or PEG had higher ejection pressures. The PEG results were consistent with observations made during actual pressing, where it was obvious that additional lubrication was needed. A significant increase in ejection pressure was seen with higher compaction pressures.

The PTC was used to determine the axial strength of the compacts. The axial strength of the green PTC compacts is measured by crushing the compacts along the pressing face. For these tests, it gives a rough measure of the green strength of the puck during handling but gives a better measure of the strength during ejection from the press. High values for axial strength are desired since it should correlate to good green strength of the puck and gives a relative measure of the likelihood of cracking due to stress induced during the ejection of the puck. Figure 7 of Appendix G contains the available data. The data for the powders was inconsistent when comparing within a binder test. Therefore, no general trends could be discerned. For the most part, strength increased with increasing pressure as expected.

The cohesiveness of the green compact represents a ratio between the axial green strength of the compact and the maximum friction pressure between the compact and die walls. This parameter is directly related to cracking and lamination resistance during compact ejection. When less than one, the compacts will likely develop cracks during ejection unless special care is implemented. If above 1 , cracking during removal from the die should not occur under normal conditions. The cohesiveness was measured using the PTC, and the data is plotted in Figure 8 of Appendix G. About half of the binder systems 
WSRC-TR-2001-00454

Revision 0

produced powders with acceptable cohesiveness. Some scatter was seen in the data so generalizations could not be made.

Hold-Up

A significant concern with the granulation process for plant production is the amount of hold-up in the vessel since this clean-up operation will have to be performed remotely. The amount of hold-up for each test was measured and is reported in Appendix D and E. Target hold-up was considered to be less than $5 \%$. General observations about hold-up included the following: spraying PEG solutions increased the amount of hold-up; spraying PEG combined with HMPC had inconsistent results with test using $8 \%$ HPMC $/ 20 \%$ PEG solution producing very little hold-up; spraying Duramax solutions resulted in minimal hold-up; and spraying Airvol or PEG solutions on Airvol containing powders resulted in low hold-up amounts. Limited testing was performed to determine whether the hold-up continued to build-up at a constant rate or whether it would reach a saturation limit as seen with the attritor. The preliminary results showed that hold-up continued to accumulate; however enough repetitions were not done to determine whether a saturation limit could be or had been reached due to the suspension of the program.

Hold-up also played a considerable role in the determination of the maximum capacity. As mentioned earlier, significant problems were seen with flow of the powder with higher powder amounts. Transfer of the powder out of the vessel was hampered by the large amount of powder; thus, hold-up was very high. The data reported in Appendix D does not properly reflect the amount of hold-up in the vessel with respect to free-released powder. After initial attempts to freely transfer the powder, the sides of the vessel were agitated to help with removal of the powder. The amount of powder remaining after that step is what is reflected in Appendix D.

The use of the agitator impacted the hold-up seen in the vessel. The amount was slightly higher and the hold-up was harder to remove. This was likely due to the amount of binder and powder that were thrown at the sides of the vessel at a higher velocity, thus creating more hold-up.

\section{CONCLUSIONS}

Based on the results of the granulation matrix testing, the following observations and conclusions were made:

- $8 \mathrm{wt} \%$ Airvol 21-205 added to the precursors followed by spraying a 10\% Airvol solution on the powder during granulation was the optimal binder system based on all of the data. The higher Airvol 21-205 addition to the precursors gave better results than the $6 \mathrm{wt} \%$ addition, and the higher concentration solution also seemed to produce better results than the $5 \%$ Airvol solution.

- Good powders and pucks were produced when Duramax 1023 was added to the precursors and water was added during granulation, but this product is no longer available. Duramax 1020 did not have the same qualities. Solutions of Duramax 1020, 
when added during granulation, did result in good powders and pucks, but several problems were seen with pumping this solution because of the solids content.

- The maximum capacity was determined to be consistent with the vendor recommendations.

- The agitator was determined to be unnecessary to produce adequate powders and furthermore resulted in additional hold-up, so it was removed on March 14, 2000.

- Additional lubricants were not needed with the recommended binder system (i.e., Airvol), but would be required with some of the other binder systems (e.g., PEG).

- Drying was determined not to be necessary because the flowability and pressing characteristics of the powder were considered acceptable without ancillary drying.

- As with previous uranium testing, uranium powders had better flow properties and lower dust levels but possibly had higher hold-up. The optimal binder system (i.e., Airvol in the powder and Airvol sprayed on the powder) had equivalent hold-up when tested with uranium.

\section{FUTURE CONSIDERATIONS}

The following items should be considered in any future plant-scale testing:

- Nozzle pattern was not well tested and should be re-visited to minimize maintenance and over-spray problems during plant operation. A fan shaped nozzle was used throughout the testing; however, it has to be operated in a specific direction for effective granulation to occur. This could create a problem for maintenance in the plant and increases the potential for upsets. Preliminary testing with a cone-shaped nozzle while spraying water gave good results and should provide better binder distribution and less potential for upset.

- The spray arm location and nozzle position should be further evaluated in the largerscale equipment since this greatly affects granule formation and the amount of hold-up. It needs to be optimized for the vessel size.

- The binder rate of addition needs to be optimized to the pump system used in the plant. Nominal addition rate for the lab-scale testing was $80 \mathrm{~g} / \mathrm{min}$ to $100 \mathrm{~g} / \mathrm{min}$ but will depend on the binder and spray nozzle design.

- The optimal vessel speed, along with process limits, need to be defined for the plantscale system. Data from the lab-scale and the PuCTF testing should be used as a starting point.

- The optimal processing time, along with the process limits, need to be defined for the plant-scale system. Data from the lab-scale and the PuCTF testing should be used as a starting point.

- The maximum powder processing temperature needs to be defined after mixing in the plant-scale attritor. This was not thoroughly examined in the matrix testing but was shown to have an effect on steam generation and product hold-up in the granulator.

- The rate of accumulation/hold-up in the granulator needs to be defined for plant prototypical processing conditions. This will allow for a general idea of the required maintenance and will help define the extent of engineering that needs to be performed to define the cleaning equipment/process for the granulator. 
WSRC-TR-2001-00454

Revision 0

- The granulation equipment has not been tested with plutonium. However, uranium testing has shown promising results with the equipment, and similar tumble granulation methods have been used in the past to make full-scale plutonium pucks in the Pu Facility at LLNL.

- Once parameters are optimized for the baseline formulation, a suite of impurity tests needs to be done to further evaluate the effects of impurities on granulation (e.g., effects of hygroscopic impurities).

\section{ACKNOWLEDGEMENTS}

The work described in this report was a concerted effort by many people involved in the Plutonium Immobilization Program. The testing could not have been completed without the assistance and hard work of the following people:

- Frank L. Beckett, LLNL

- William E. Biehl, Retired LLNL employee

- Al Fontes, LLNL

- David T. Herman, WSRC

- Manny Herrera, LLNL

- David E. Hiland, WSRC

- Joe Phillips, LLNL

- Darrell K. Pugh, LLNL

- Steve Williams, Former LLNL employee

\section{REFERENCES}

NMTP/IP-99-003, Rev. 0, "Process Development Testing for FYOO”, C.C. Herman, December 15, 1999.

NMTP/IP-99-003, Rev. 1, "Process Development Testing for FYOO”, C.C. Herman, February 14, 2000.

LLNL Procedure TIP-PD-02, Revision 0, "Fabricating Surrogate Plutonium Ceramic Pucks - Plant Prototypical Process", C.C. Herman, April 3, 2000.

PIP-TP-98-001, "Research and Development Task Plan: Plutonium Immobilization Form and Process Development", C.A. Cicero-Herman, March 23, 1998.

R.M. German, "Powder Metallurgy Science", $2^{\text {nd }}$ edition, The Pennsylvania State University, Metal Powders Industry Foundation, NJ, 1994.

\section{APPENDICES}

A Synopsis from Process Development Matrix for FY00 - Status as of April 2001

B Granulation Test Series A - Binder Type, Amount, and Addition Point

C Granulation Test Series B - Binder Type, Amount, and Addition Point with $\mathrm{Hf} / \mathrm{Ce} / \mathrm{U}$ Formula

D Summary of Cerium Granulation Test Parameters and Product Data

E Summary of Uranium Granulation Test Parameters and Product Data

F Puck Data for Granulation Matrix Testing

G Powder Test Center Data for Granulation Matrix Testing 
Appendix A - Synopsis from Process Development Studies for FY00 - Status as of April 2001

\begin{tabular}{|c|c|c|c|c|c|c|c|}
\hline $\begin{array}{c}\text { Test } \\
\text { Series }\end{array}$ & Parameter Studied & Variable/Test & $\begin{array}{l}\text { Responsible } \\
\text { Site }\end{array}$ & $\begin{array}{c}\text { Target } \\
\text { Completion } \\
\text { Date }\end{array}$ & Material & Acceptance Criteria & Status \\
\hline \multirow{12}{*}{ GA } & \multirow{11}{*}{$\begin{array}{l}\text { Binder Type, Amount, } \\
\text { and Addition Point (3 kg } \\
\text { Batch) }\end{array}$} & PEG 8K & LLNL & \multirow{12}{*}{ Complete } & $\mathrm{Hf} / \mathrm{Ce} / \mathrm{Ce}$ & \multirow{12}{*}{$\begin{array}{l}\text { Formation of granules, sieve } \\
\text { classification, powder test center } \\
\text { analyses, and flow and dust } \\
\text { testing. Pucks as applicable for } \\
\text { green strength, handling, } \\
\text { cracking, and density. }\end{array}$} & \multirow{12}{*}{$\begin{array}{l}\text { Testing has shown that the following binders } \\
\text { (with addition point listed) produce acceptable } \\
\text { granulated material without impacting the } \\
\text { process: 3\% PEG - Prec.; 20\% PEG Solution - } \\
\text { Gran.; } 4.5 \text { to 6\% Airvol - Prec.; } 5 \text { or } 10 \% \text { Airvol } \\
\text { Solution - Gran.; } 8 \text { - 10\% HPMC solution - } \\
\text { Gran.; } 10 \text { - 20\% PEG solution - Gran.; } 3 \text { \& 5\% } \\
\text { Duramax - Prec.; and } 5 \text { \& } 10 \% \text { Duramax } \\
\text { solution - Gran. Airvol in precursors with } \\
\text { additional airvol added during granulation was } \\
\text { deemed the baseline process from this testing. } \\
\text { Results will be documented during closeout. }\end{array}$} \\
\hline & & Airvol 21-205 & LLNL & & $\mathrm{Hf} / \mathrm{Ce} / \mathrm{Ce}$ & & \\
\hline & & Airvol 205 & LLNL & & $\mathrm{Hf} / \mathrm{Ce} / \mathrm{Ce}$ & & \\
\hline & & PEG 8K/HPMC E-5 & LLNL & & $\mathrm{Hf} / \mathrm{Ce} / \mathrm{Ce}$ & & \\
\hline & & PEG 8K/Airvol 21-205 & LLNL & & $\mathrm{Hf} / \mathrm{Ce} / \mathrm{Ce}$ & & \\
\hline & & PEG 8K/Airvol 205 & LLNL & & $\mathrm{Hf} / \mathrm{Ce} / \mathrm{Ce}$ & & \\
\hline & & Maltrin M-150 & LLNL & & $\mathrm{Hf} / \mathrm{Ce} / \mathrm{Ce}$ & & \\
\hline & & Duramax & SRTC/ LLNL & & $\mathrm{Hf} / \mathrm{Ce} / \mathrm{Ce}$ & & \\
\hline & & Added to Precursors & LLNL & & $\mathrm{Hf} / \mathrm{Ce} / \mathrm{Ce}$ & & \\
\hline & & $\begin{array}{l}\text { Sprayed during } \\
\text { Granulation }\end{array}$ & LLNL & & $\mathrm{Hf} / \mathrm{Ce} / \mathrm{Ce}$ & & \\
\hline & & Added to Attritor & LLNL & & $\mathrm{Hf} / \mathrm{Ce} / \mathrm{Ce}$ & & \\
\hline & Full Batch & Verify Best of Binders & LLNL & & $\mathrm{Hf} / \mathrm{Ce} / \mathrm{Ce}$ & & \\
\hline GB & Hf/Ce/U Verification & Verify Best of Binders & LLNL & Complete & $\mathrm{Hf} / \mathrm{Ce} / \mathrm{U}$ & Acceptable granules \& pucks. & Consistent results with GA testing. \\
\hline GC & Hf/Ce/Mn Verification & Verify Best of Binders & LLNL & Not performed & $\mathrm{Hf} / \mathrm{Ce} / \mathrm{Mn}$ & Acceptable granules. & Not performed. \\
\hline GD & Maximum Capacity & 8,12 , vs $16 \mathrm{qt}$ & LLNL & Complete & $\mathrm{Hf} / \mathrm{Ce} / \mathrm{Ce}$ & \begin{tabular}{|} 
Formation of granules, sieve \\
classification, powder test center \\
analyses, and flow and dust \\
testing. Pucks as applicable. \\
Vessel hold-up. \\
\end{tabular} & $\begin{array}{l}\text { Current size ratio seems optimal. } \\
\text { However, larger size batches were shown } \\
\text { to also produce acceptable product. } \\
\text { Biggest problem was discharge. }\end{array}$ \\
\hline \multirow[b]{2}{*}{ GE } & \multirow[b]{2}{*}{ Agitator } & No Agitator & LLNL & Complete & \multirow[b]{2}{*}{$\mathrm{Hf} / \mathrm{Ce} / \mathrm{Ce}$} & \multirow{2}{*}{$\begin{array}{l}\text { Formation of granules, sieve } \\
\text { classification, powder test center } \\
\text { analyses, and flow and dust } \\
\text { testing. Pucks as applicable. } \\
\text { Vessel hold-up. } \\
\end{array}$} & $\begin{array}{c}\begin{array}{c}\text { Baseline process established without use of } \\
\text { agitator. }\end{array} \\
\end{array}$ \\
\hline & & During binder addition & LLNL & Complete & & & $\begin{array}{c}\text { With the agitator, larger and harder } \\
\text { granules were produced and additional } \\
\text { hold-up was seen. }\end{array}$ \\
\hline \multirow{4}{*}{ GF } & \multirow{4}{*}{ Binder/Water Addition } & Type/Size of Nozzle & LLNL/SRTC & On-going & \multirow{3}{*}{$\mathrm{Hf} / \mathrm{Ce} / \mathrm{Ce}$} & \multirow{3}{*}{$\begin{array}{c}\text { Formation of granules, sieve } \\
\text { classification, powder test center } \\
\text { analyses, and flow and dust } \\
\text { testing. Pucks as applicable. } \\
\text { Vessel hold-up. } \\
\end{array}$} & \multirow{4}{*}{$\begin{array}{c}\text { Preliminary nozzles, position, and rate of } \\
\text { addition have been defined. However, the } \\
\text { final decision will be based on full-plant } \\
\text { size equipment. Additional optimization } \\
\text { will be necessary. }\end{array}$} \\
\hline & & Nozzle Position & LLNL/SRTC & On-going & & & \\
\hline & & Rate of Addition & LLNL/SRTC & On-going & & & \\
\hline & & Verify with Uranium & LLNL & On-going & $\mathrm{Hf} / \mathrm{Ce} / \mathrm{U}$ & Acceptable Granules \& Pucks & \\
\hline \multirow{4}{*}{ GG } & \multirow{4}{*}{$\begin{array}{c}\text { Lubricant type and } \\
\text { amount }\end{array}$} & No Additional & LLNL/SRTC & Complete & \multirow{3}{*}{$\mathrm{Hf} / \mathrm{Ce} / \mathrm{Ce}$} & \multirow{4}{*}{$\begin{array}{c}\text { Flow and dust testing, Powder } \\
\text { test center analyses, puck ejection } \\
\text { force, and acceptable pucks. }\end{array}$} & \multirow{3}{*}{$\begin{array}{c}\text { No additional lube was deemed necessary } \\
\text { after pressing in the PuCTF press with } \\
\text { baseline powders. }\end{array}$} \\
\hline & & EBS, Ca Stearate, others & LLNL/SRTC & Not performed & & & \\
\hline & & dry vs liquid & LLNL/SRTC & Not performed & & & \\
\hline & & Verify with Uranium & LLNL & Complete & $\mathrm{Hf} / \mathrm{Ce} / \mathrm{U}$ & & U results were similar for Carver press. \\
\hline \multirow{2}{*}{$\mathrm{GH}$} & \multirow{2}{*}{ Drying Necessity } & Formulator & LLNL & $4 / 30 / 00$ & $\mathrm{Hf} / \mathrm{Ce} / \mathrm{Ce}$ & \multirow{2}{*}{$\begin{array}{c}\text { Formation and flow of granules, } \\
\text { acceptable pucks. }\end{array}$} & \multirow{2}{*}{$\begin{array}{l}\text { The baseline process produced acceptable } \\
\text { results that made drying unnecessary. }\end{array}$} \\
\hline & & Vacuum (air) & LLNL & Not performed & $\mathrm{Hf} / \mathrm{Ce} / \mathrm{U}$ & & \\
\hline
\end{tabular}


Appendix A - Synopsis from Process Development Studies for FY00 - Status as of April 2001

\begin{tabular}{|c|c|c|c|c|c|c|c|}
\hline $\begin{array}{c}\text { Test } \\
\text { Series }\end{array}$ & Parameter Studied & Variable/Test & $\begin{array}{l}\text { Responsible } \\
\text { Site }\end{array}$ & $\begin{array}{c}\text { Target } \\
\text { Completion } \\
\text { Date } \\
\end{array}$ & Material & Acceptance Criteria & Status \\
\hline \multirow{4}{*}{ GI } & \multirow{4}{*}{ Vessel Speed (min, max) } & During binder addition & LLNL & $5 / 31 / 00$ & \multirow{3}{*}{$\mathrm{Hf} / \mathrm{Ce} / \mathrm{Ce}$} & \multirow{3}{*}{$\begin{array}{c}\text { Formation of granules, sieve } \\
\text { classification, powder test center } \\
\text { analyses, and flow and dust } \\
\text { testing. Pucks as applicable. } \\
\text { Vessel hold-up. }\end{array}$} & \multirow{3}{*}{$\begin{array}{l}\text { Vessel speed has been defined for the } \\
\text { current scale equipment, will have to be } \\
\text { further optimized for the plant-scale } \\
\text { equipment when testing is performed. }\end{array}$} \\
\hline & & During tumbling & LLNL & Complete & & & \\
\hline & & $\begin{array}{c}\text { Determine Operating } \\
\text { Limits }\end{array}$ & $\begin{array}{l}\text { LLNL/ } \\
\text { SRTC }\end{array}$ & On-going & & & \\
\hline & & Verify with Uranium & LLNL & Complete & $\mathrm{Hf} / \mathrm{Ce} / \mathrm{U}$ & Acceptable Granules \& Pucks & Results similar to Ce. \\
\hline \multirow{4}{*}{ GJ } & \multirow{4}{*}{$\begin{array}{l}\text { Time of Tumble (min, } \\
\text { max) }\end{array}$} & During binder addition & LLNL & Complete & \multirow{3}{*}{$\mathrm{Hf} / \mathrm{Ce} / \mathrm{Ce}$} & \multirow{3}{*}{$\begin{array}{c}\text { Formation of granules, sieve } \\
\text { classification, powder test center } \\
\text { analyses, and flow and dust } \\
\text { testing. Pucks as applicable. }\end{array}$} & \multirow{3}{*}{$\begin{array}{l}\text { Process time has been defined for the } \\
\text { current scale equipment, will have to be } \\
\text { further optimized for the plant-scale } \\
\text { equipment when testing is performed. }\end{array}$} \\
\hline & & During tumbling & LLNL & Complete & & & \\
\hline & & $\begin{array}{l}\text { Determine Operating } \\
\text { Limits }\end{array}$ & $\begin{array}{l}\text { LLNL/ } \\
\text { SRTC }\end{array}$ & On-going & & & \\
\hline & & Verify with Uranium & LLNL & Complete & $\mathrm{Hf} / \mathrm{Ce} / \mathrm{U}$ & Acceptable Granules \& Pucks & Results similar to Ce. \\
\hline GK & Powder Temperature & Impact on Process & $\begin{array}{l}\text { LLNL/ } \\
\text { SRTC }\end{array}$ & On-going & $\mathrm{Hf} / \mathrm{Ce} / \mathrm{Ce}$ & Formation of granules. & $\begin{array}{l}\text { Need to evaluate maximum temperature } \\
\text { allowed to make acceptable product and } \\
\text { minimize hold-up. }\end{array}$ \\
\hline
\end{tabular}


Appendix B - Granulation Test Series A - Binder Type, Amount, and Addition Point

\begin{tabular}{|c|c|c|c|c|}
\hline Test \# & Binder Type & \begin{tabular}{|c|}
$\begin{array}{c}\text { Binder Amount (Based on Total } \\
\text { Feed) }\end{array}$ \\
\end{tabular} & Addition Point & Batch ID \\
\hline GA-1 & \multirow{2}{*}{ Airvol 21-205 } & $4.5 \mathrm{wt} \%$ liquid ( $21 \%$ solids) & \multirow{2}{*}{ Precursors } & 112499 \\
\hline GA-2 & & $6 \mathrm{wt} \%$ liquid ( $21 \%$ solids) & & 112299,030800 \\
\hline GA-3 & \multirow{3}{*}{ Airvol 21-205 } & $5 \%$ Solution & \multirow{3}{*}{ Granulation } & 112399 \\
\hline GA-4 & & $10 \%$ Solution & & $\begin{array}{c}111599-0 \text { to }-2,092899-0 \&-1,113099-0 \&-4 \\
011100-0 \text { \& }-2,031500-2,020400-0 \&-1,032000 \\
0 \&-1 \\
\end{array}$ \\
\hline GA-5 & & 15\% Solution & & $\begin{array}{c}113099-2 \& \text {-3, 021100-0\&-1, 022800-0, 111799 } \\
032100-4,032000-0 \text { \& }-3,031500,022500-5 \\
022900-3\end{array}$ \\
\hline GA-6 & \multirow{5}{*}{ Airvol 205} & $1 \mathrm{wt} \%$ dry & \multirow{5}{*}{ Attritor } & NA \\
\hline GA-7 & & $2 w t \%$ dry & & 111899-0 \& -1, 121499-0 \\
\hline GA-8 & & $3 \mathrm{wt} \%$ dry & & 120799-0 \& -1, 121499-3 \\
\hline GA-9 & & $4 \mathrm{wt} \%$ dry & & $120799-3$ \\
\hline GA-10 & & $5 \mathrm{wt} \%$ dry & & NA \\
\hline GA-11 & \multirow{3}{*}{ PEG 8K } & $4 w^{2} \%$ & \multirow{3}{*}{ Precursors } & $121399-0$ to -2 \\
\hline GA-12 & & $5 \mathrm{wt} \%$ & & NA \\
\hline GA-13 & & $3 \mathrm{wt} \%$ & & $122299-0$ \& $-1,122799-0$ \& -1 \\
\hline GA-14 & \multirow{5}{*}{ PEG 8K } & $10 \%$ Solution & \multirow{5}{*}{ Granulation } & $120899-0 \&-1$ \\
\hline GA-15 & & $20 \%$ Solution & & $120899-2$ to $-4,010400-0$ \\
\hline GA-16 & & $30 \%$ Solution & & $010400-1 \&-3$ \\
\hline GA-17 & & $25 \%$ Solution & & $010500-0$ \\
\hline GA-18 & & $15 \%$ Solution & & $122799-1,011100-2 \&-3$ \\
\hline GA-19 & \multirow{3}{*}{$\begin{array}{l}\text { Duramax } 1020 \\
\quad \text { or } 1023\end{array}$} & $3 w t \%$ liquid & \multirow{3}{*}{ Precursors } & $011000-0$ to -3 \\
\hline GA-20 & & $5 \mathrm{wt} \%$ liquid & & $011800-0$ \& $-2,022500-0$ \& -1 \\
\hline GA-21 & & $7 \mathrm{wt} \%$ liquid & & $022500-3 \&-4$ \\
\hline GA-22 & \multirow{3}{*}{ Duramax 1020} & $5 \%$ Solution & \multirow{3}{*}{ Granulation } & $121799-1$ to -3 \\
\hline GA-23 & & $10 \%$ Solution & & \begin{tabular}{|c|}
$111799-1$ \& -2, 010400-0, 021700-5, 011100-2, \\
$020100-0$ to $-3,010500-1$ \& $-3,120899,011800-0$ \\
$012500-0,020700-0,021100-1,030300-3,031500$ \\
$0,040300-0 \&-1$ \\
\end{tabular} \\
\hline GA-24 & & $15 \%$ Solution & & $\begin{array}{c}1207990,-2 \&-3,111899-1,032100-0,-1, \&-4, \\
032000-3 \&-4\end{array}$ \\
\hline GA-25 & \multirow{4}{*}{$\begin{array}{l}\text { PEG 8K/ } \\
\text { HPMC E-5 }\end{array}$} & $5 \%$ HPMC Sol'n/10\% PEG Sol'n & \multirow{4}{*}{ Granulation } & $010500-1 \&-3$ \\
\hline GA-26 & & $10 \%$ HPMC Sol'n $n / 10 \%$ PEG Sol'n & & $010400-2,120899-1,011100-0$ \\
\hline GA-27 & & 5\% HPMC Sol'n/20\% PEG Sol'n & & NA \\
\hline GA-28 & & 8\% HPMC Sol'n/20\% PEG Sol'n & & $\begin{array}{c}120799-1,111899-0,121499-1 \& \text {-3, 010500-0 \& } \\
2,032100-2 \&-3,010400-1 \&-3,113099-2 \\
121799,100799-3\end{array}$ \\
\hline GA-29 & \multirow{4}{*}{ Airvol 21-205 } & $4.5 \%$ Airvol liquid $/ 5 \%$ Sol'n & \multirow{4}{*}{$\begin{array}{l}\text { Precursors/ } \\
\text { Granulation }\end{array}$} & $011800-5,112499-1$ \\
\hline GA-30 & & $4.5 \%$ Airvol liquid $/ 10 \%$ Sol'n & & $011900-0$ to $-2,020400-2 \&-3$ \\
\hline GA-31 & & $6 \%$ Airvol liquid $/ 5 \%$ Sol'n & & $012500-0,-1,-2, \&-4$ \\
\hline GA-32 & & $6 \%$ Airvol liquid $/ 10 \%$ Sol'n & & $013100-0$ to $-3,020700-2 \&-3,030800-1$ to -4 \\
\hline GA-33 & $\begin{array}{c}\text { PEG 8K/ } \\
\text { Airvol 21-205 }\end{array}$ & $2 \mathrm{wt} \%$ PEG $/ 4 \mathrm{wt} \%$ Airvol liquid & Precursors & 020100-0 to -3 \\
\hline GA-34 & \multirow{2}{*}{$\begin{array}{c}\text { PEG 8K/ } \\
\text { Airvol 21-205 }\end{array}$} & $\begin{array}{c}4.5 \mathrm{wt} \% \text { liquid Airvol/ } \\
20 \% \text { PEG Solution }\end{array}$ & \multirow{2}{*}{$\begin{array}{l}\text { Airvol to Prec./ } \\
\text { PEG in Gran. }\end{array}$} & 020400-0 \& -1 \\
\hline GA-35 & & $\begin{array}{c}6 \mathrm{wt} \% \text { liquid Airvol/ } \\
20 \% \text { PEG Solution }\end{array}$ & & 020700-0 \& -1 \\
\hline
\end{tabular}


Appendix B - Granulation Test Series A - Binder Type, Amount, and Addition Point

\begin{tabular}{|c|c|c|c|c|}
\hline Test \# & Binder Type & \begin{tabular}{|c|} 
Binder Amount (Based on Total \\
Feed)
\end{tabular} & Addition Point & Batch ID \\
\hline GA-36 & \multirow{2}{*}{$\begin{array}{c}\text { PEG 8K/ } \\
\text { Airvol 21-205 } \\
\end{array}$} & $2 \mathrm{wt} \%$ PEG/10\% Airvol sol'n & \multirow{2}{*}{$\begin{array}{l}\text { PEG to Prec./ } \\
\text { Airvol in Gran. }\end{array}$} & $021400-1 \&-2$ \\
\hline GA-37 & & $3 \mathrm{wt} \%$ PEG $/ 10 \%$ Airvol sol'n & & $122999-1,021700-0$ \& $-1,122799-1$ \\
\hline GA-38 & Maltrin M-150 & $3 \mathrm{wt} \%$ & Precursors & $021100-0 \&-1$ \\
\hline GA-39 & \multirow{4}{*}{$\begin{array}{l}\text { PEG 8K/ } \\
\text { HPMC E-5 }\end{array}$} & $2 \mathrm{wt} \%$ PEG dry $/ 5 \%$ HPMC Sol'n & \multirow{4}{*}{$\begin{array}{c}\text { PEG to } \\
\text { Precursors / } \\
\text { HPMC in } \\
\text { Granulation } \\
\end{array}$} & $021800-1$ to -3 \\
\hline GA-40 & & $2 \mathrm{wt} \%$ PEG dry $/ 8 \%$ HPMC Sol'n & & $021800-4,122299-0$ \\
\hline GA-41 & & 3 wt $\%$ PEG dry $/ 10 \%$ HPMC Sol'n & & $030200-0,-1,-3 \&-4$ \\
\hline GA-42 & & $3 \mathrm{wt} \%$ PEG dry $/ 8 \%$ HPMC Sol'n & & $022900-3,122999-0,022800-4$ \\
\hline GA-43 & Duramax 1020 & $\begin{array}{c}3 \mathrm{wt} \% \text { Duramax/ } \\
10 \% \text { Duramax Solution }\end{array}$ & $\begin{array}{l}\text { Precusors/ } \\
\text { Granulation }\end{array}$ & 022800-0 \\
\hline GA-44 & \begin{tabular}{|c|} 
Duramax $1020 /$ \\
HPMC E-5 \\
\end{tabular} & $\begin{array}{l}\mathrm{wt} \% \text { Duramax/ } \\
8 \% \text { HPMC Solution }\end{array}$ & $\begin{array}{l}\text { Precusors/ } \\
\text { Granulation } \\
\end{array}$ & 22800 \\
\hline GA-45 & Airvol 21-205 & 4.5\% Airvol liquid/ 15\% Solution & $\begin{array}{l}\text { Precusors/ } \\
\text { Granulation } \\
\end{array}$ & 020400-0 \& -1, 011900-3, 011100-2 \\
\hline
\end{tabular}


Appendix C - Granulation Test Series B - Binder Type, Amount, and Addition Point with Hf/Ce/U Formula

\begin{tabular}{|c|c|c|c|c|}
\hline Test \# & Binder Type & Binder Amount (Based on Total Feed) & Addition Point & Batch ID \\
\hline GB-1 & HPMC E-5/PEG 8K & $8 \%$ HPMC Solution/20\% PEG Solution & Granulation & $\begin{array}{c}021100-3, \\
030300-2\end{array}$ \\
\hline GB-2 & PEG 8K & 20\% PEG Solution & Granulation & $021100-5$ \\
\hline GB-3 & Airvol 21-205 & $6 \%$ Airvol & Precursors & $021400-3$ \\
\hline GB-4 & Airvol 21-205 & $6 \%$ Airvol/10\% Airvol Solution & Precursors/Granulation & $021400-5$, \\
$031400-0 ~ \&-2$ \\
\hline GB-5 & Duramax 1020 & $10 \%$ Duramax solution & Granulation & $030300-0$ \\
\hline GB-6 & PEG 8K/Airvol & $4 \%$ PEG/10\% Airvol Solution & Precursors/Granulation & $030300-3$ \\
\hline GB-7 & PEG 8K/HPMC E-5 & $4 \%$ PEG/8\% HPMC Solution & Precursors/Granulation & $030300-5$ \\
\hline GB-8 & Duramax 1020 & $6.8 \%$ Duramax & Precursors & $030900-0 ~ \&-1$ \\
\hline GB-9 & Duramax 1020/HPMC E-5 & $6.8 \%$ Duramax/10\% HPMC Solution & Precursors/Granulation & $030900-3$ \\
\hline
\end{tabular}




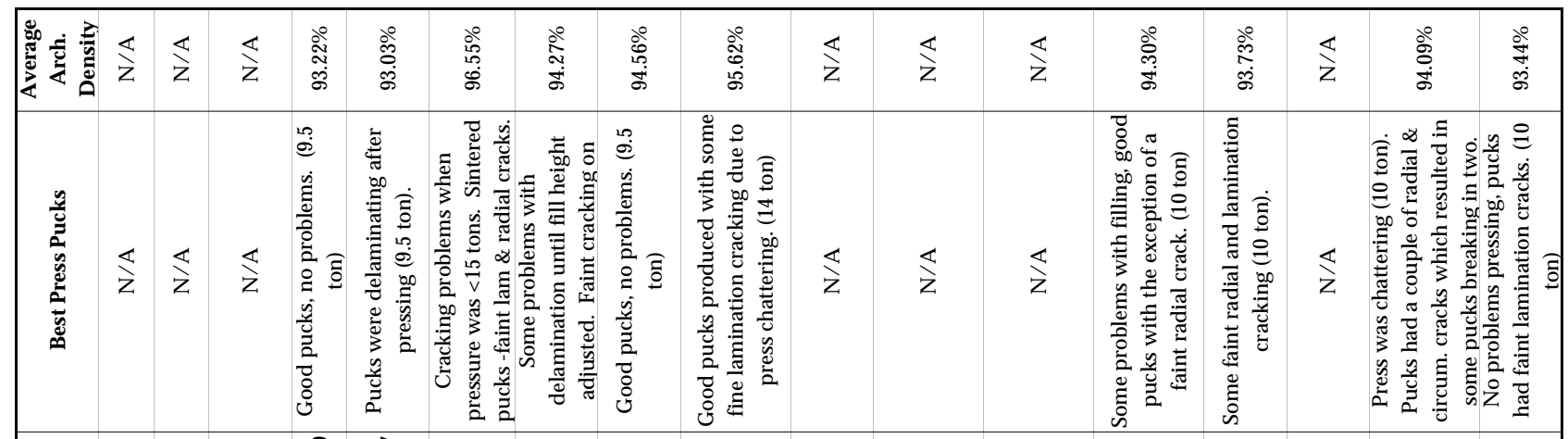

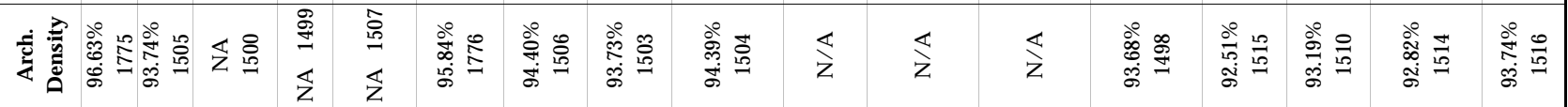

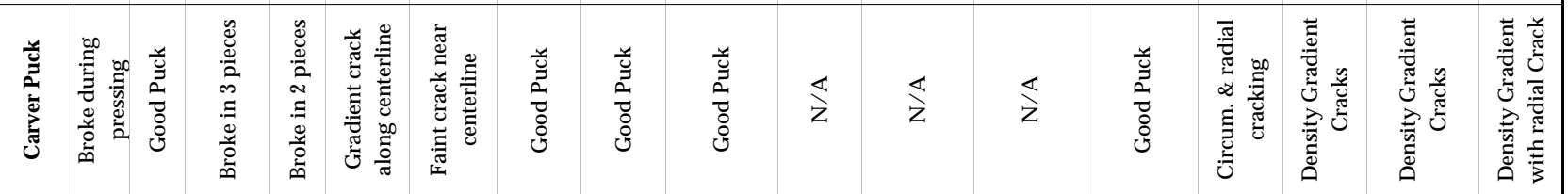

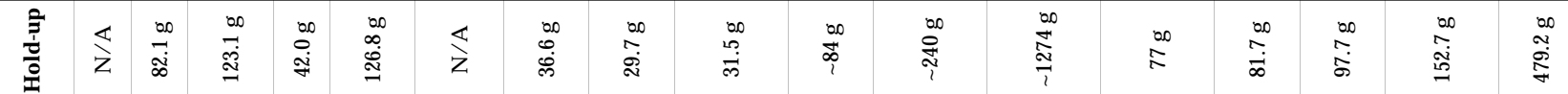

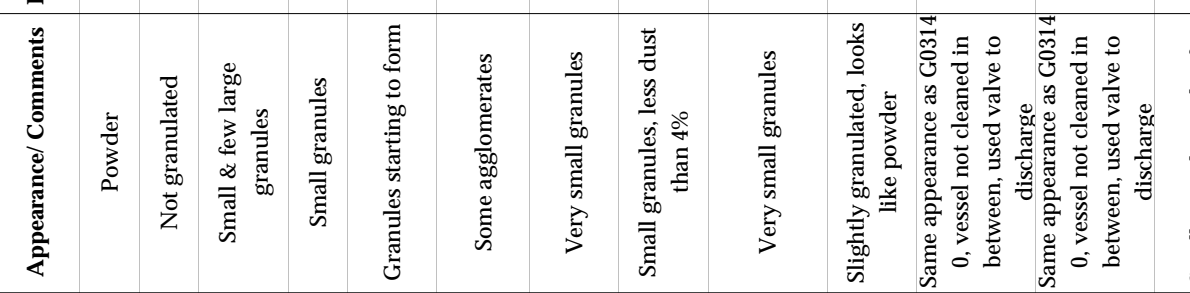

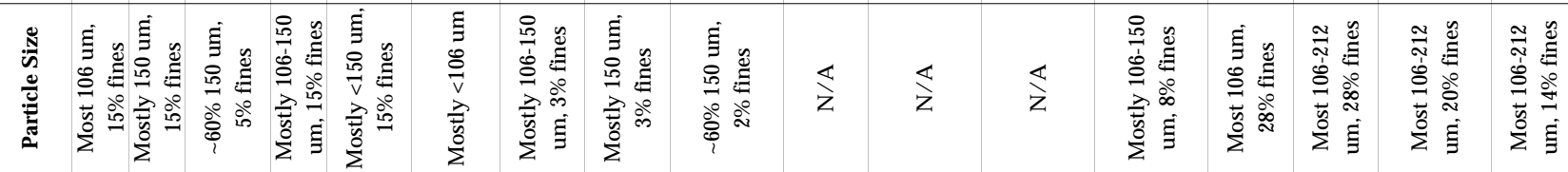

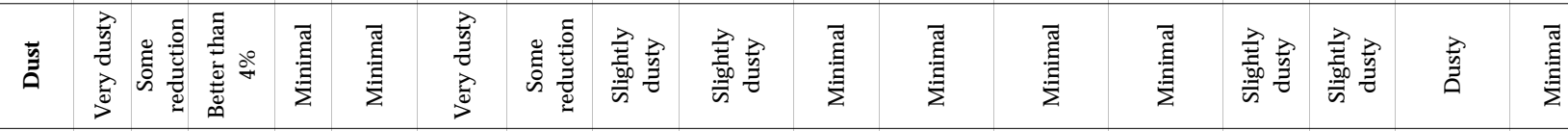

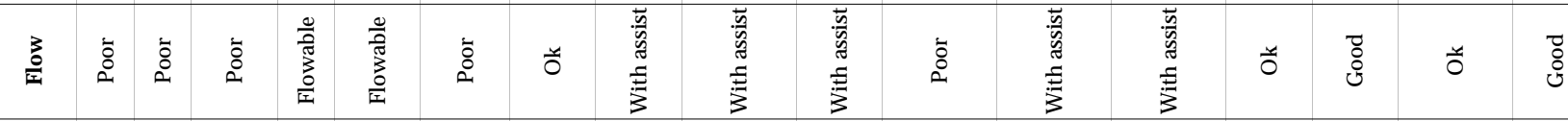

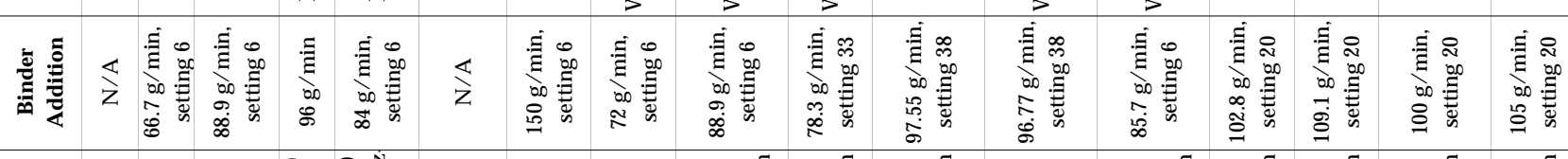

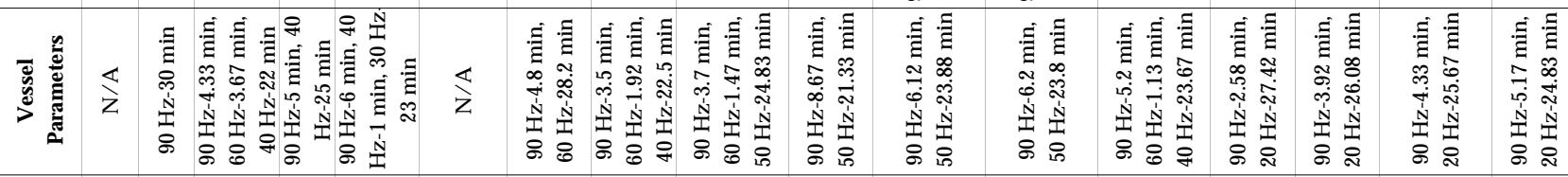

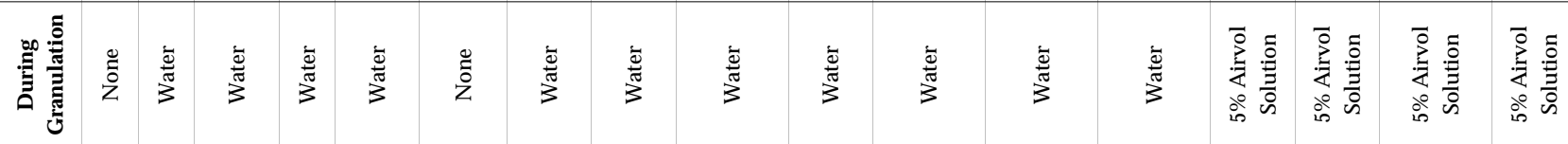

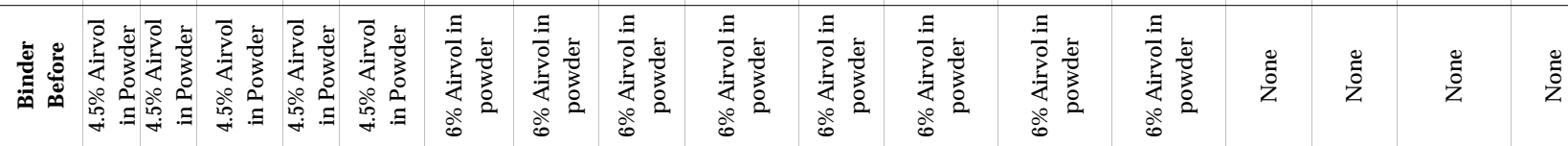

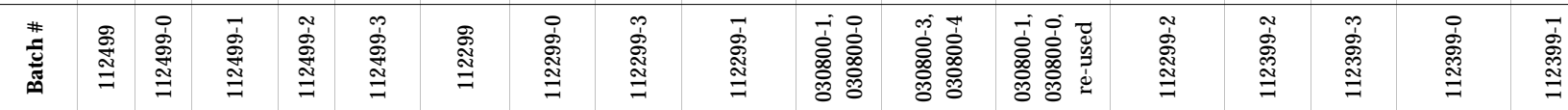

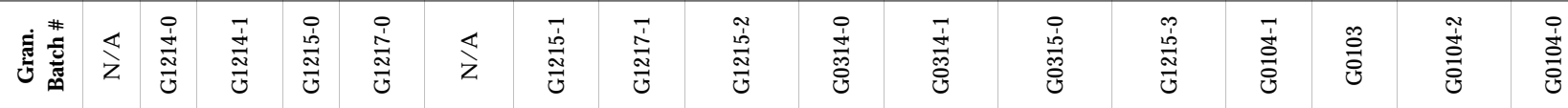

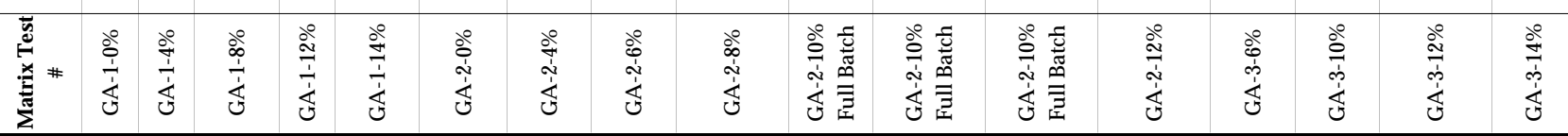




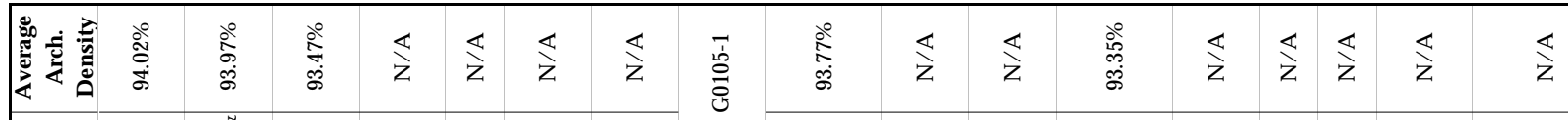

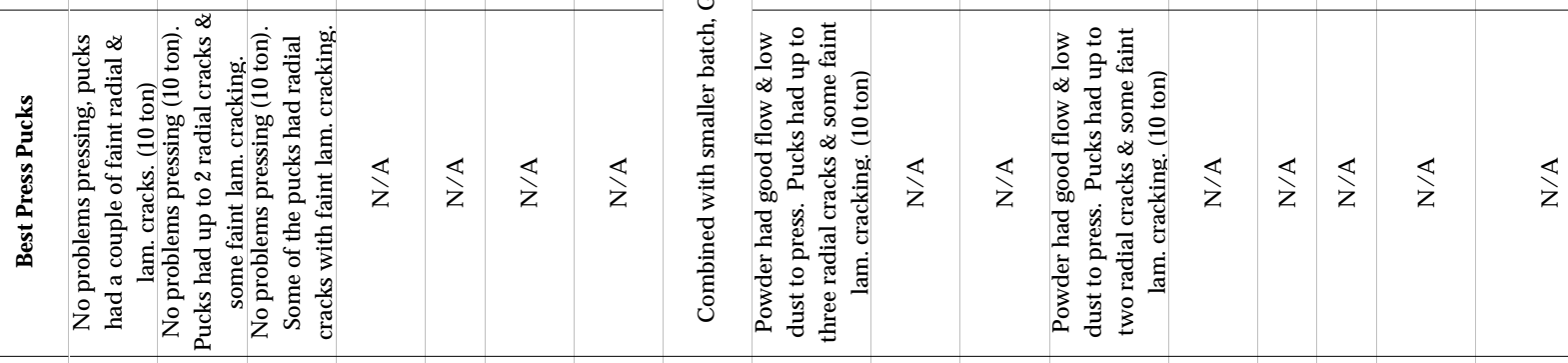

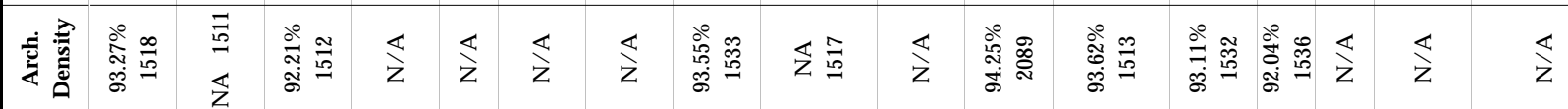

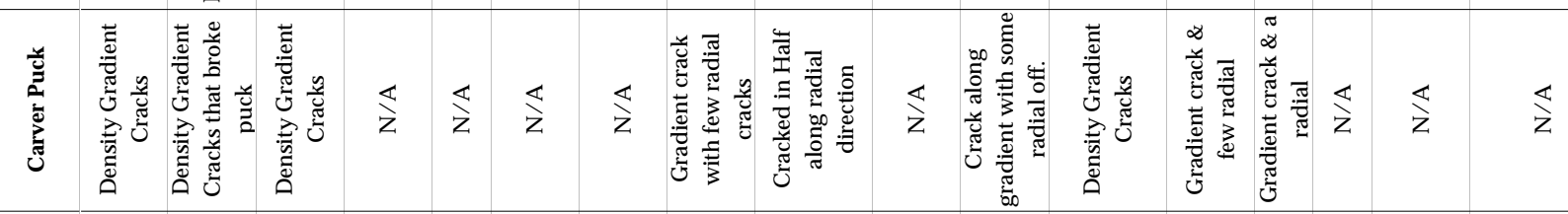

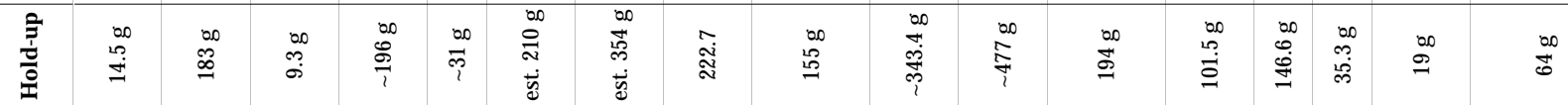

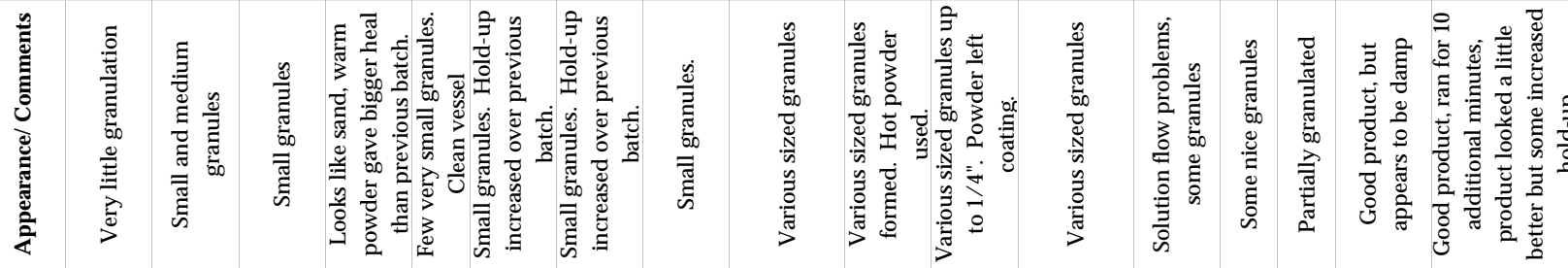

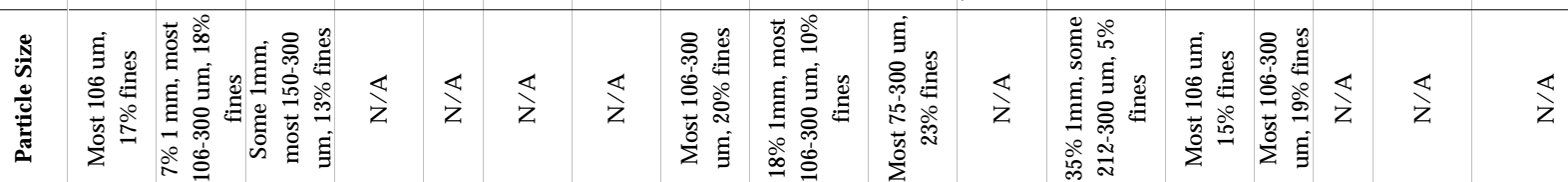

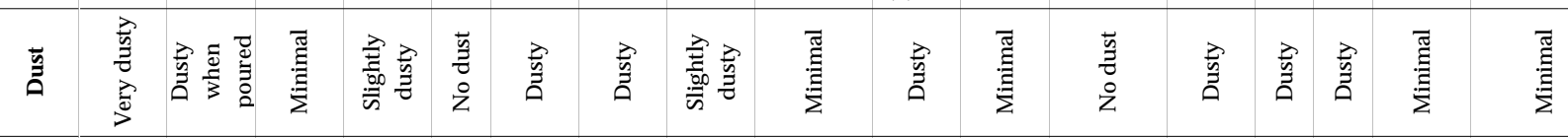

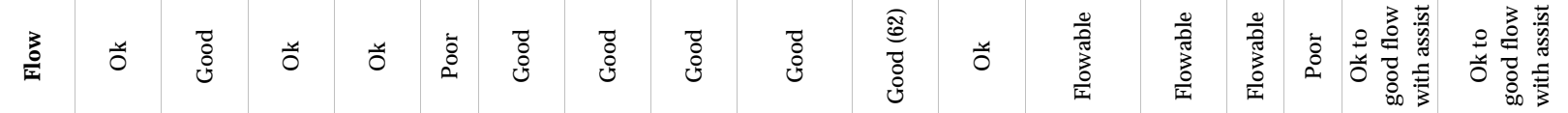

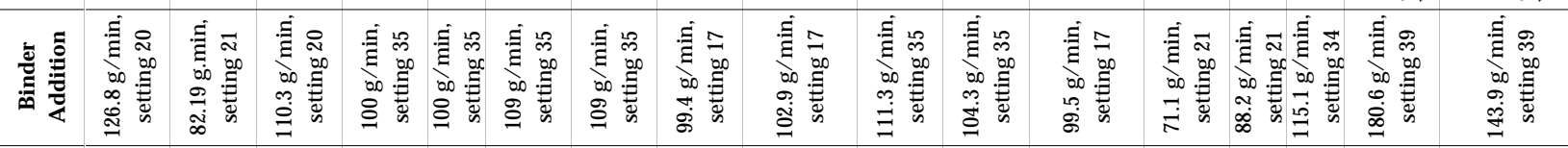

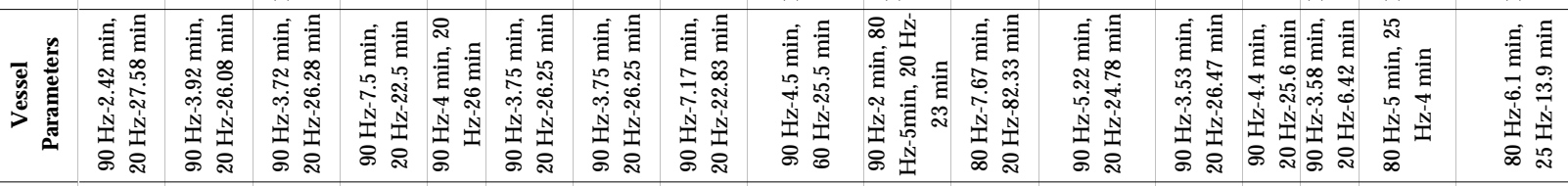

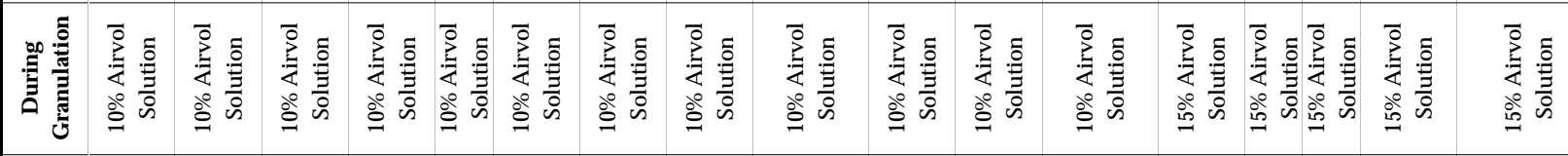

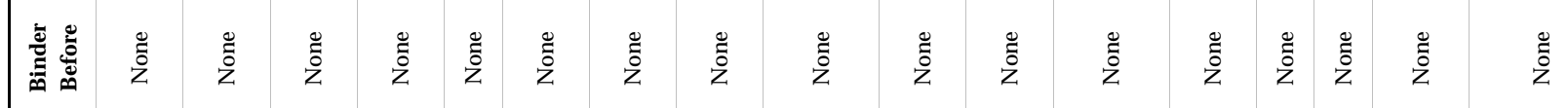

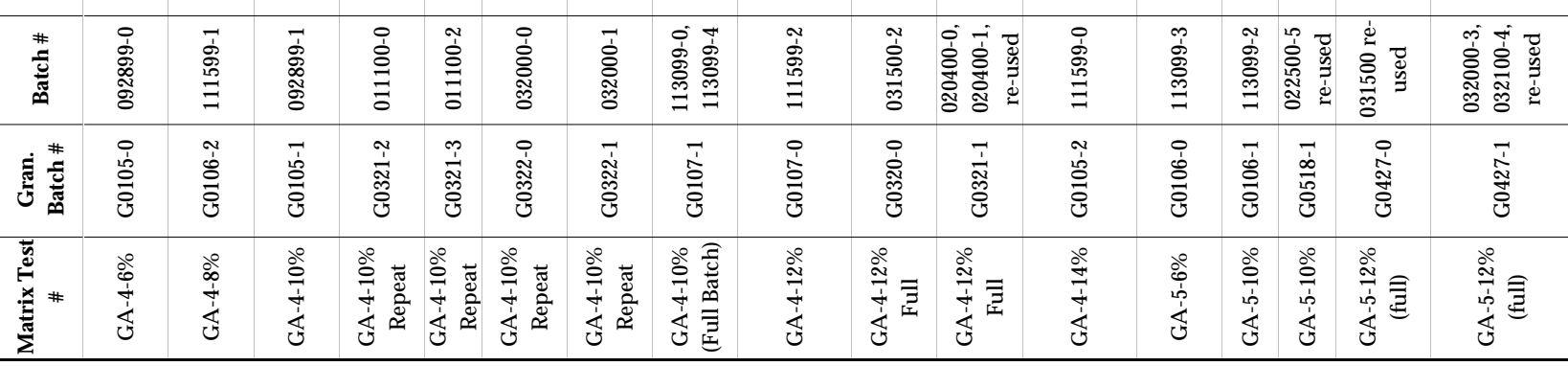




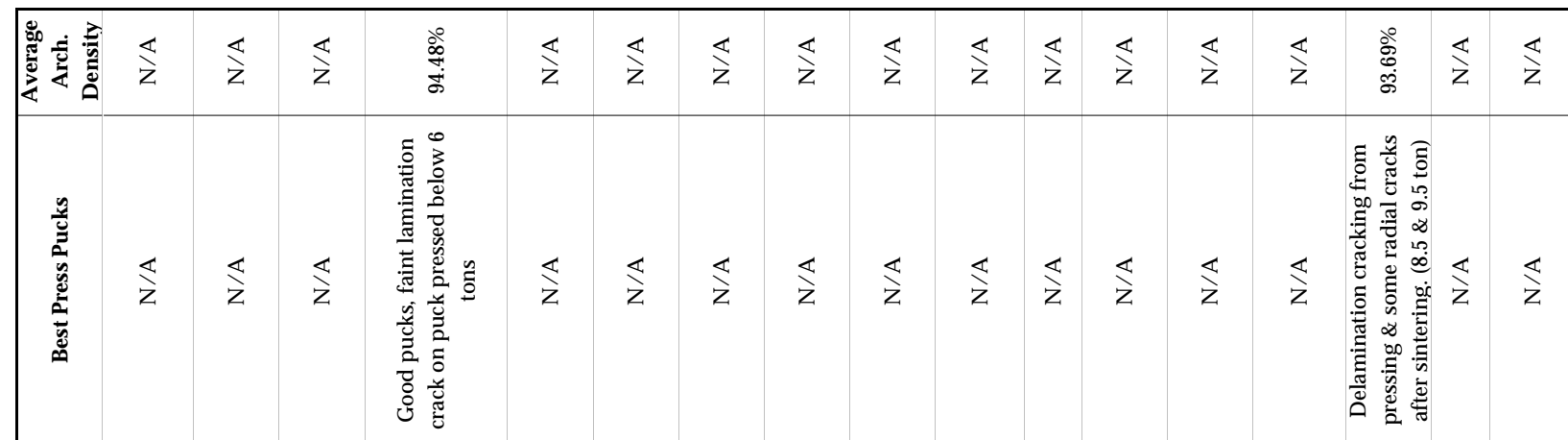

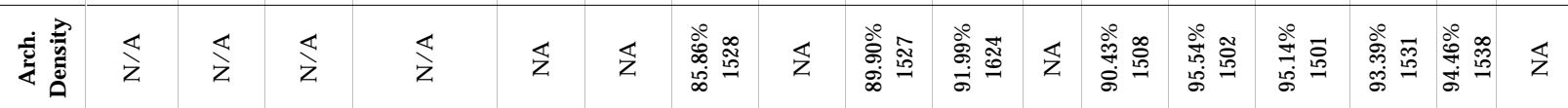

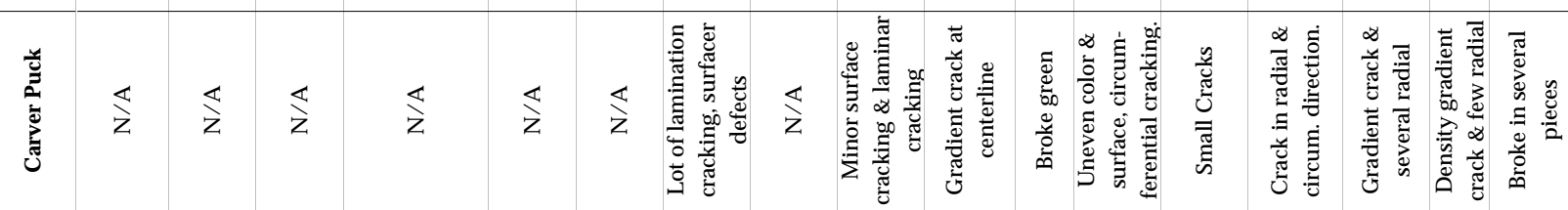

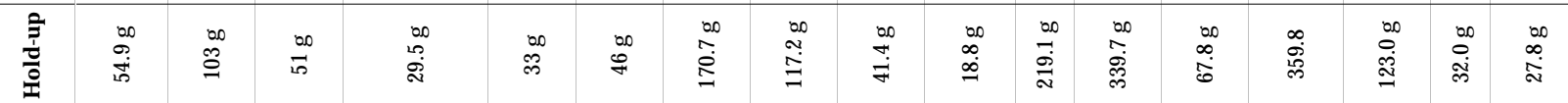

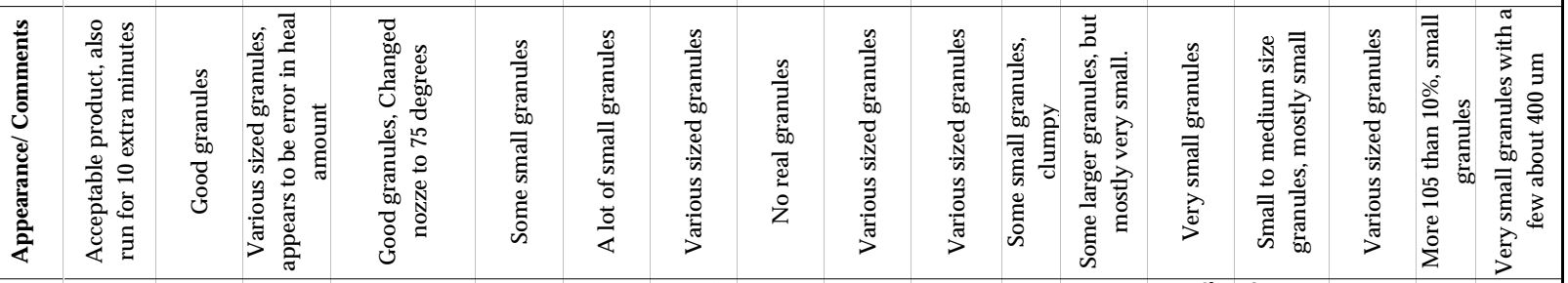

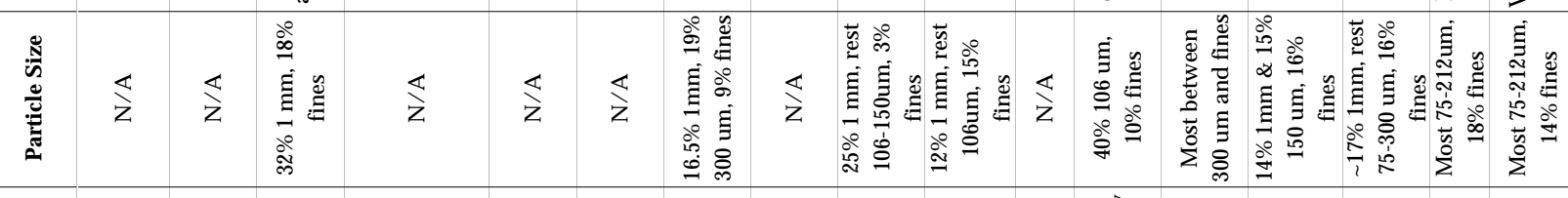

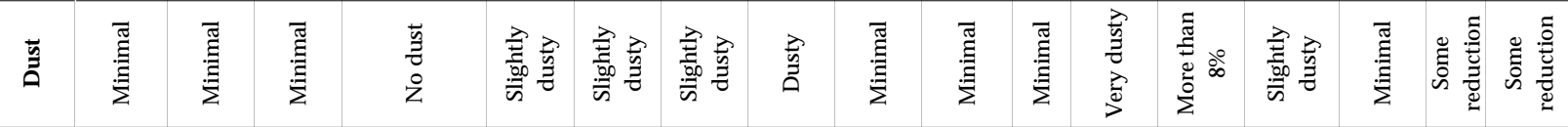

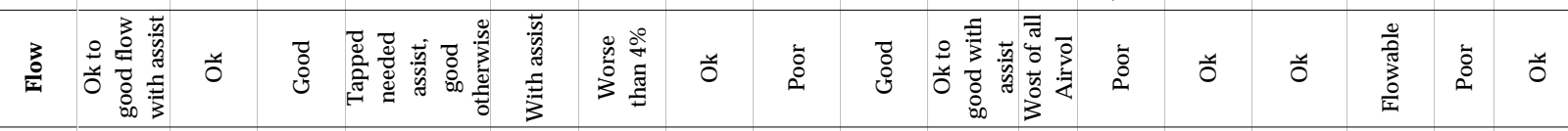

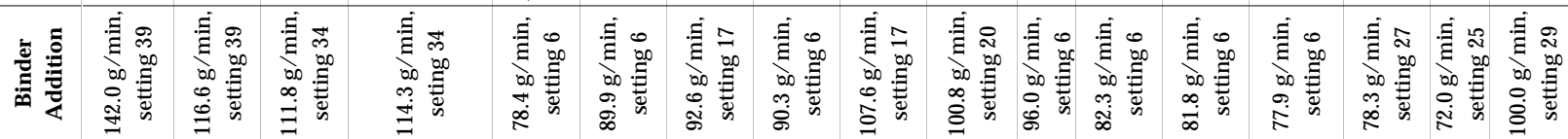

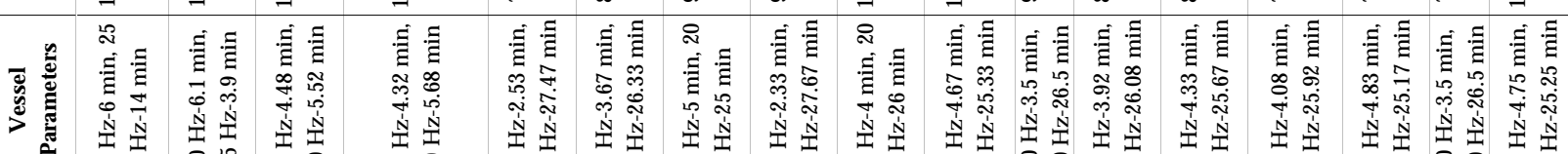

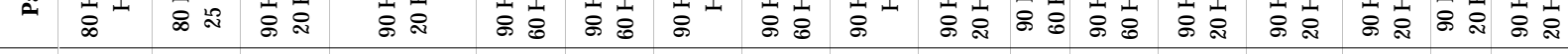

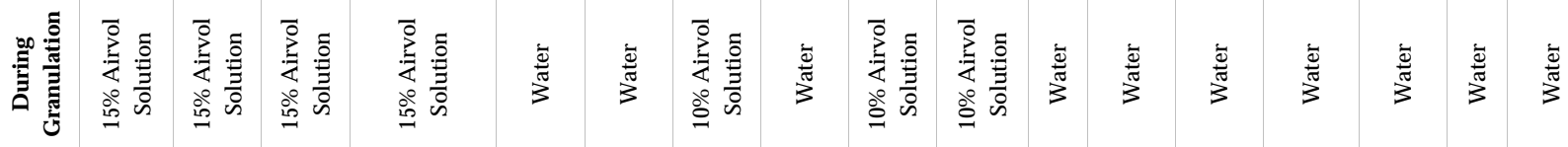

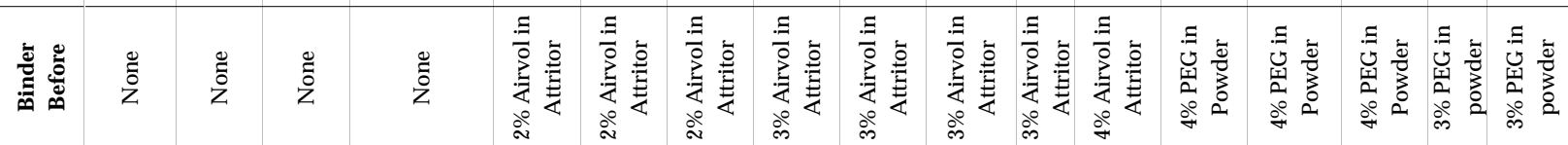

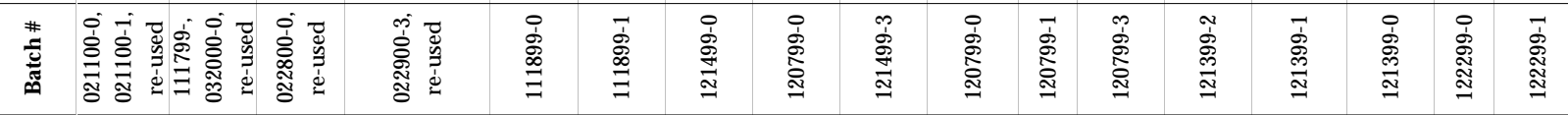

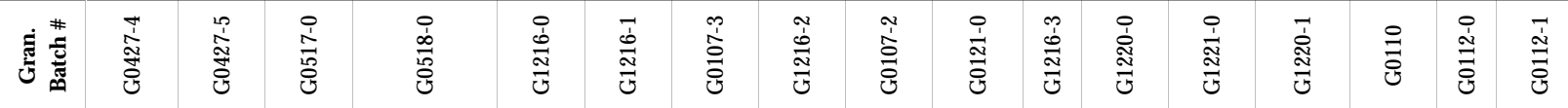

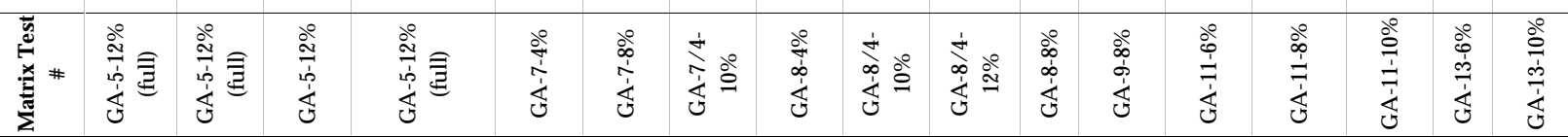




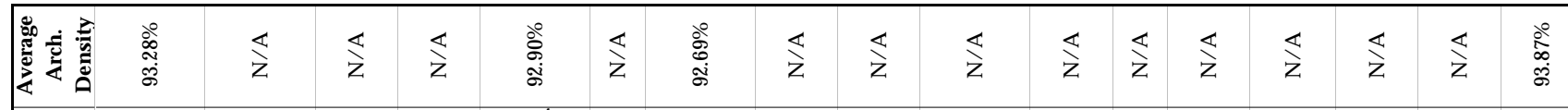

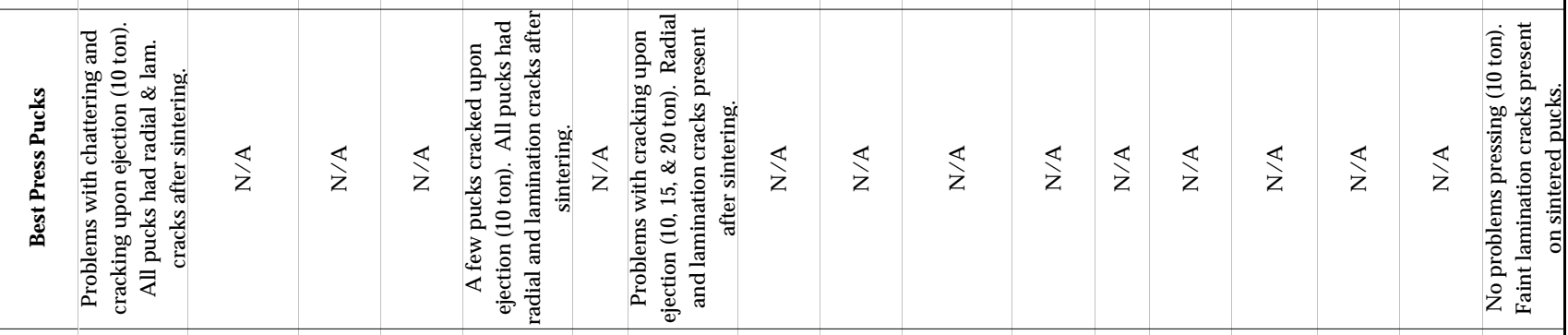

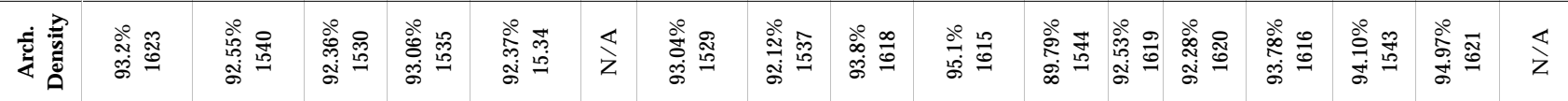

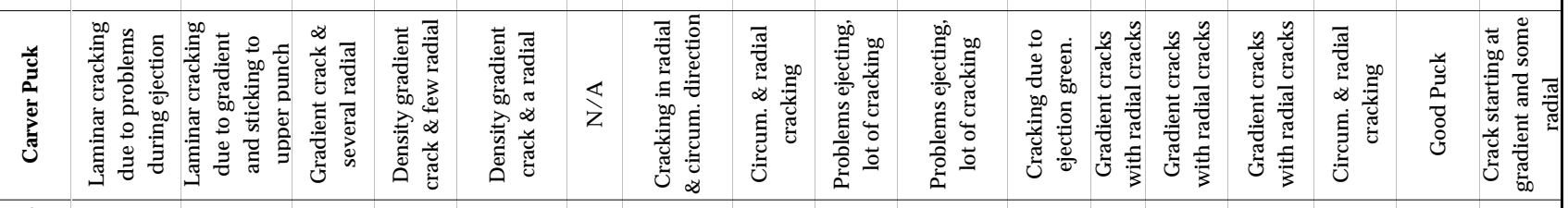

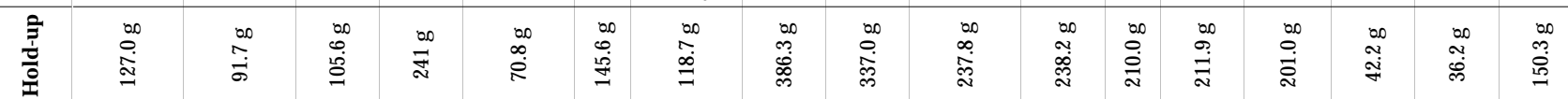

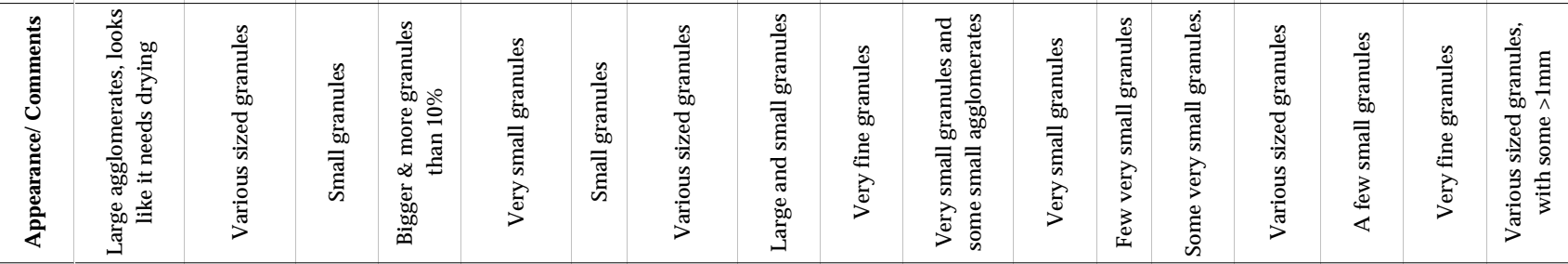

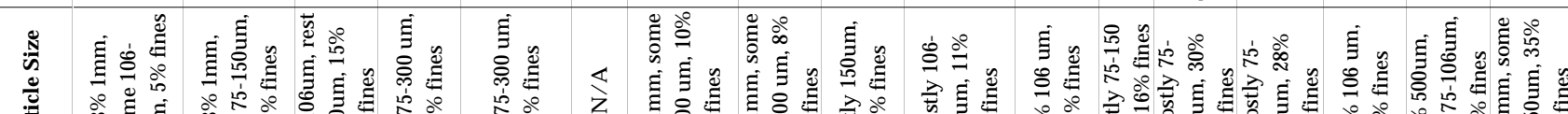

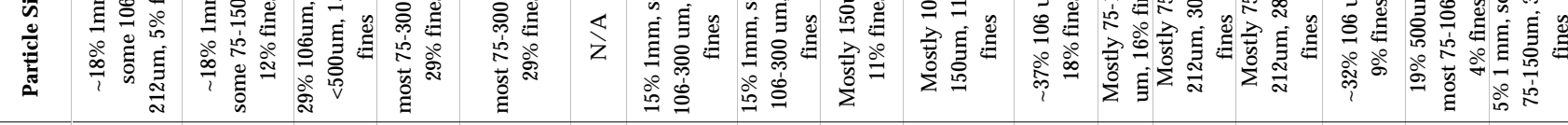

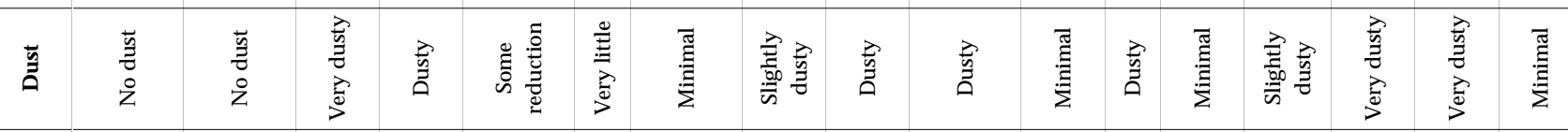

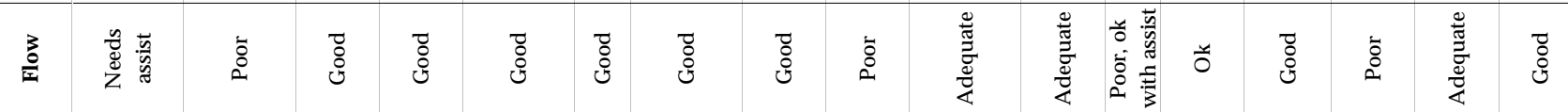

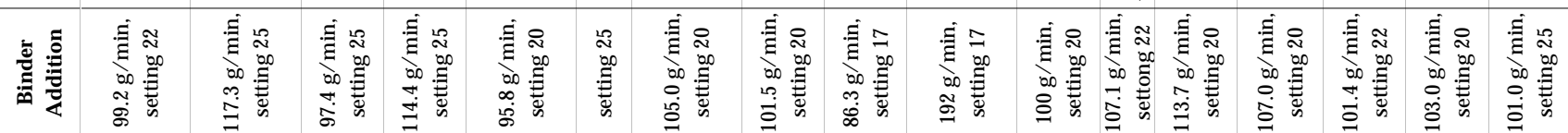

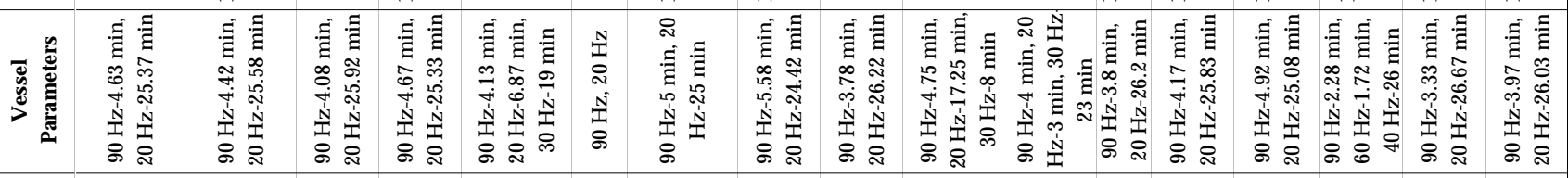

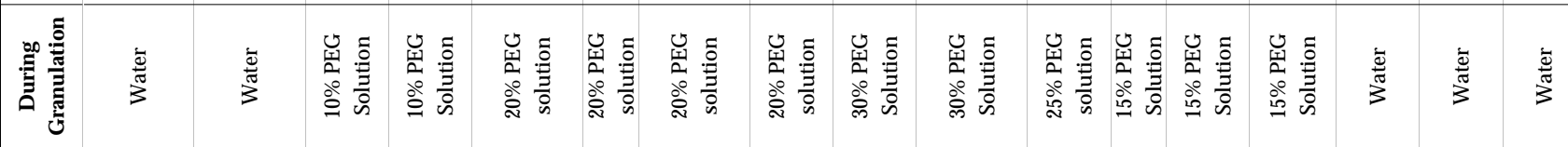

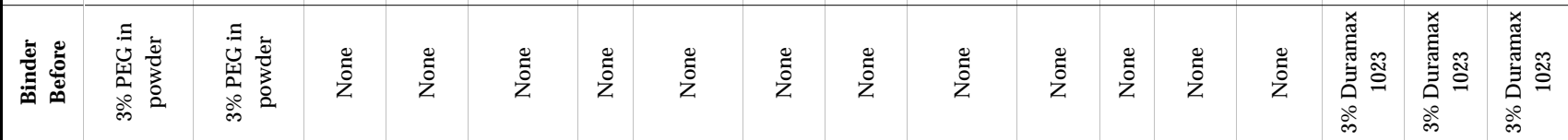

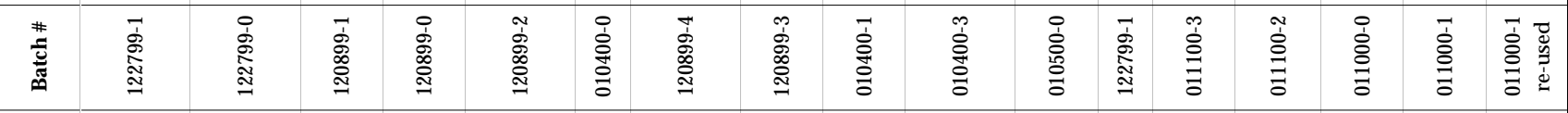

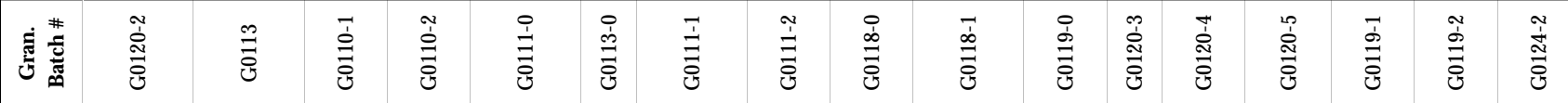

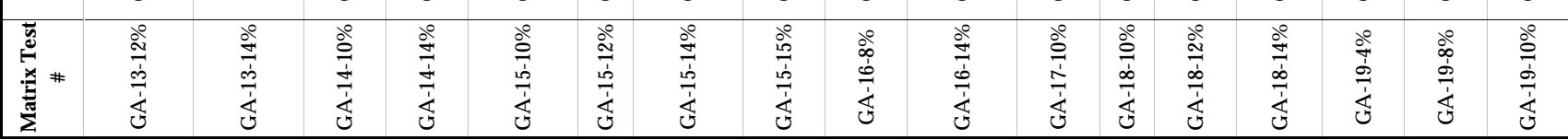




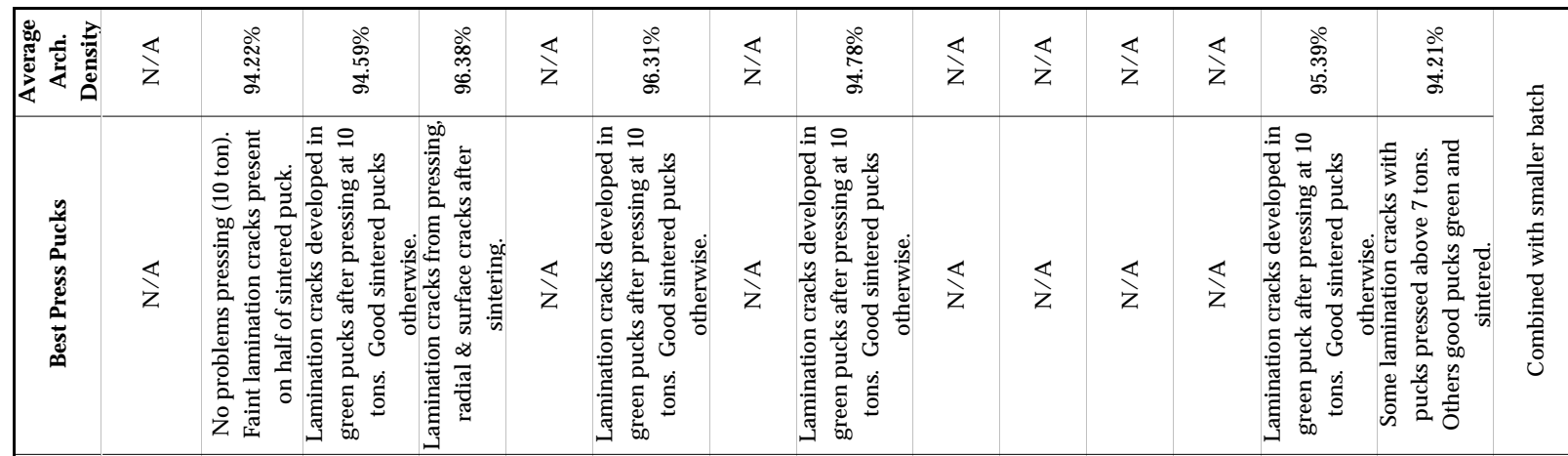

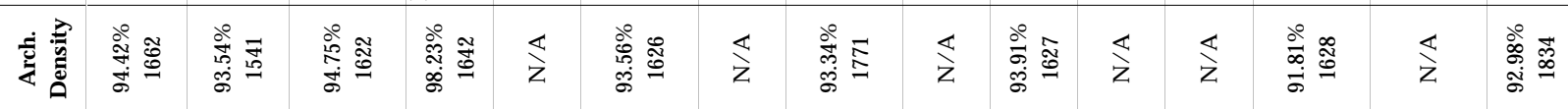

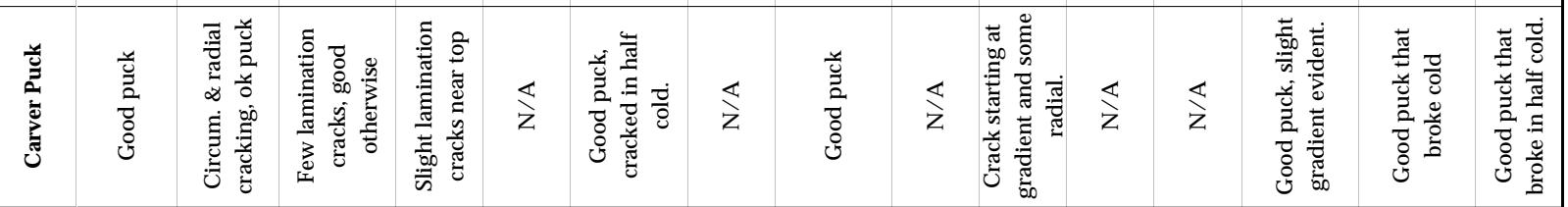

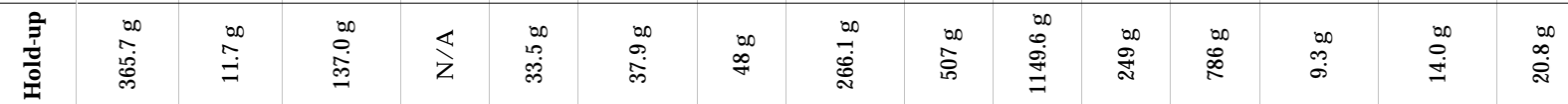
III

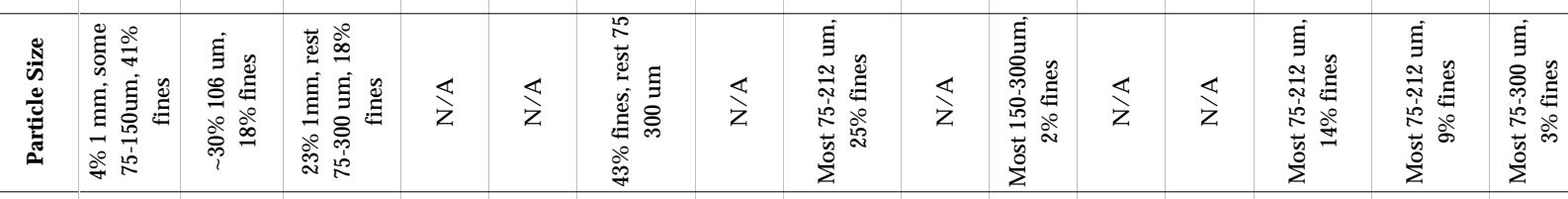

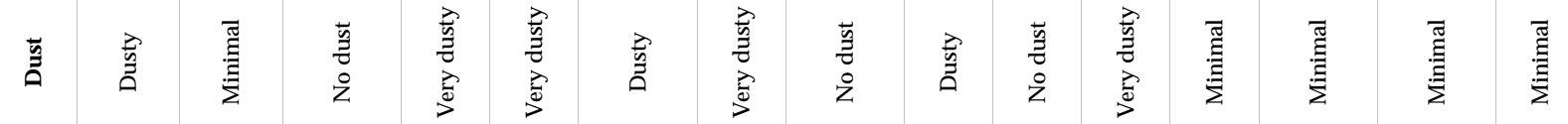

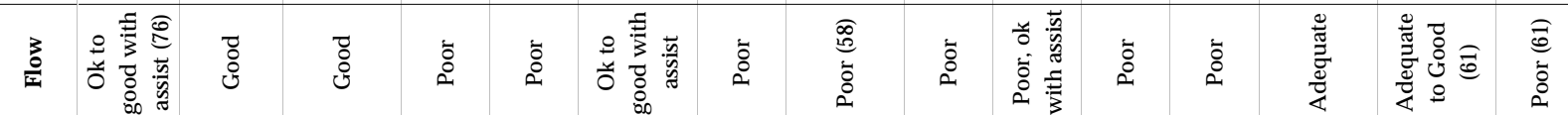
II

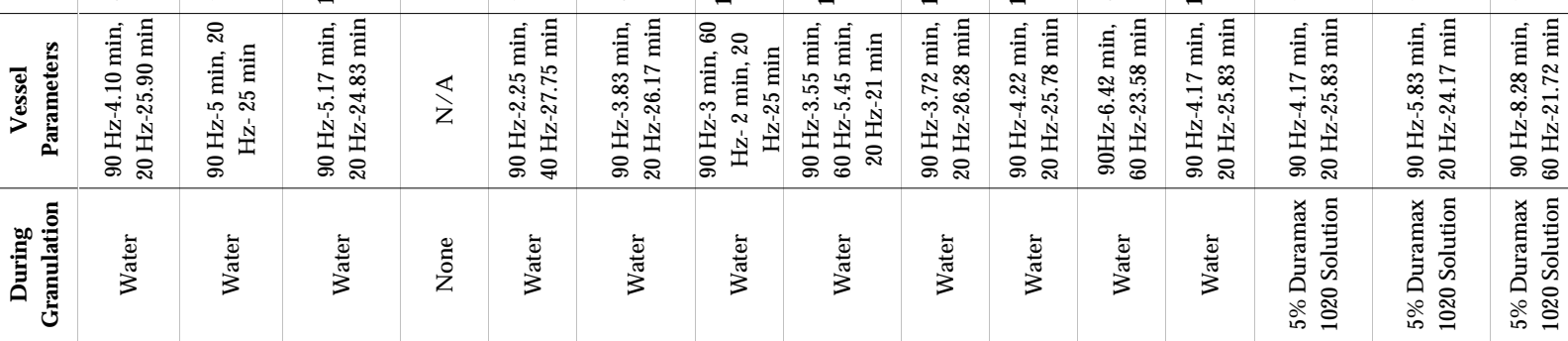

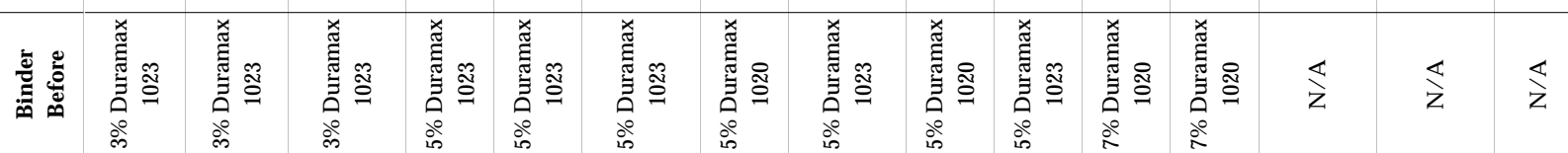

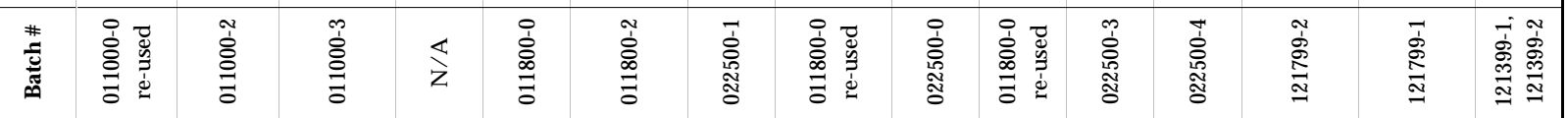

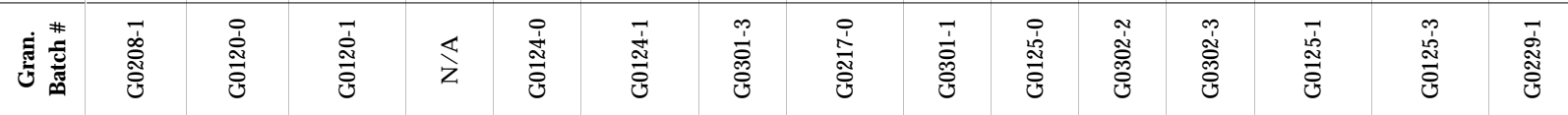

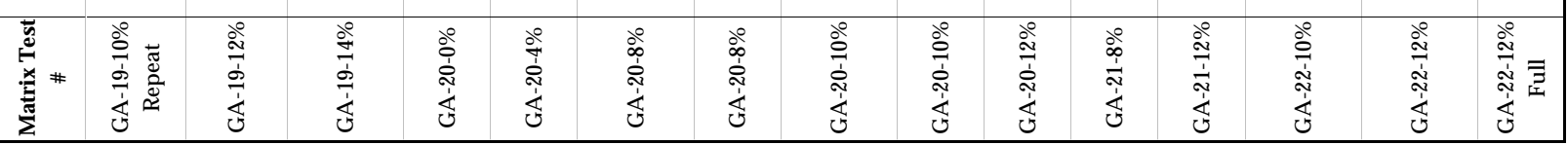




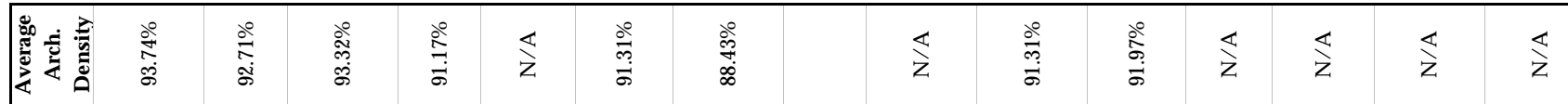

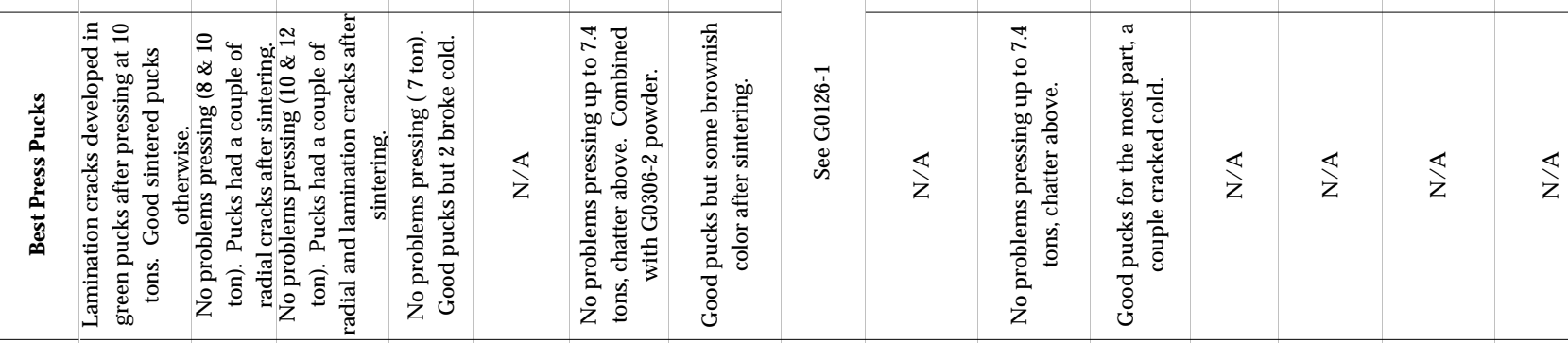

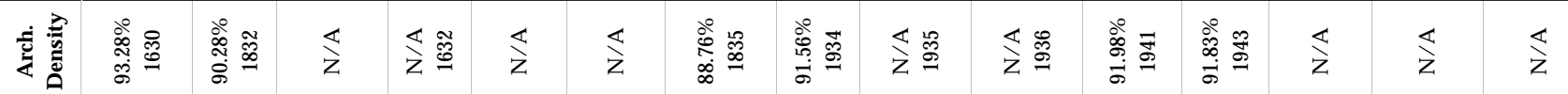

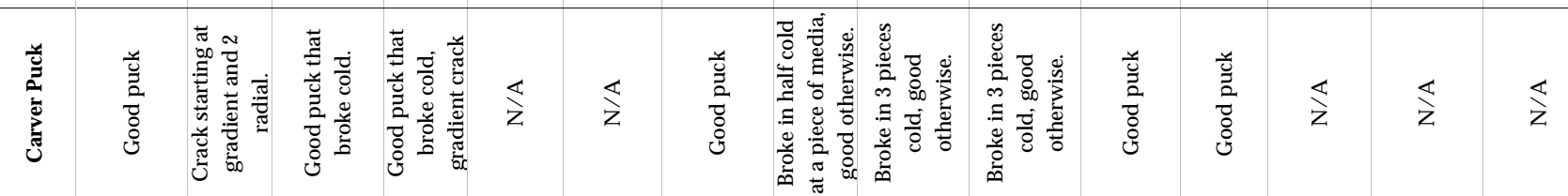

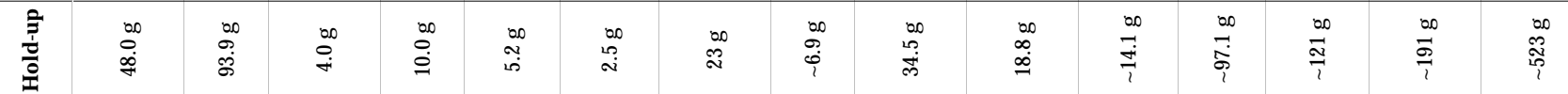

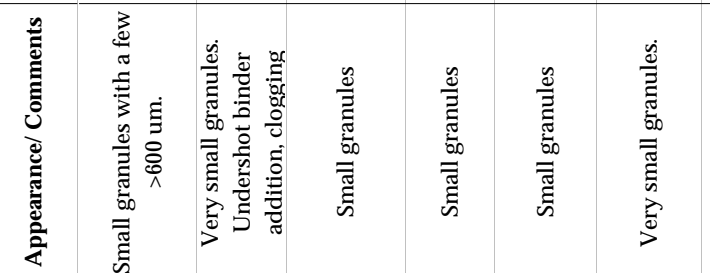

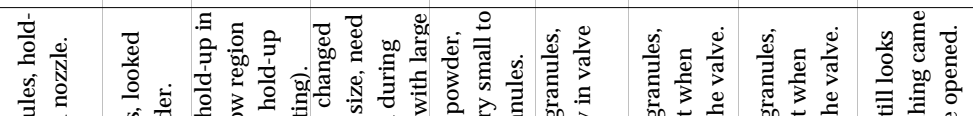

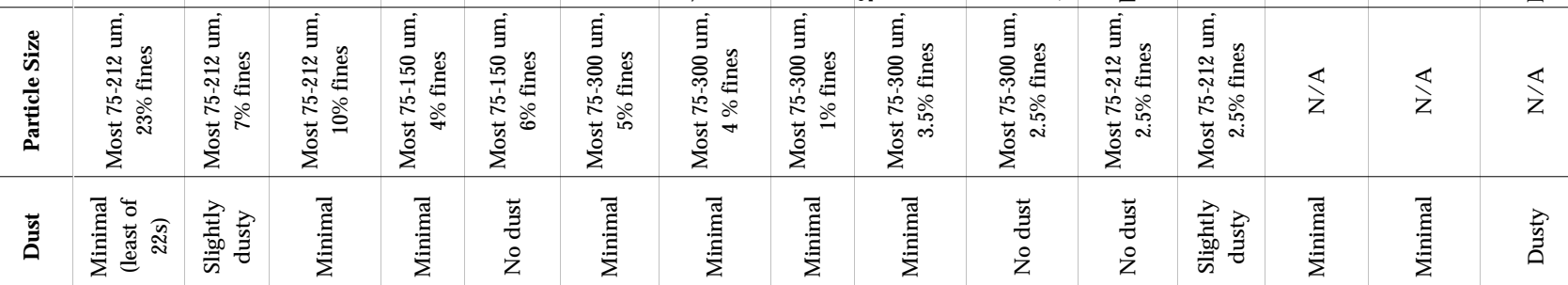

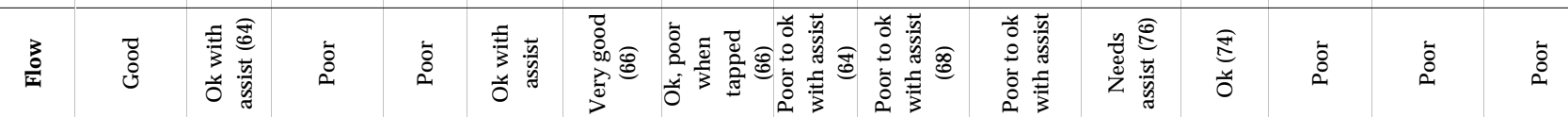

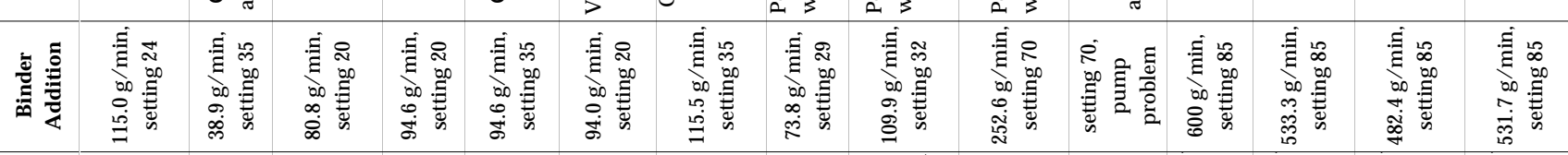

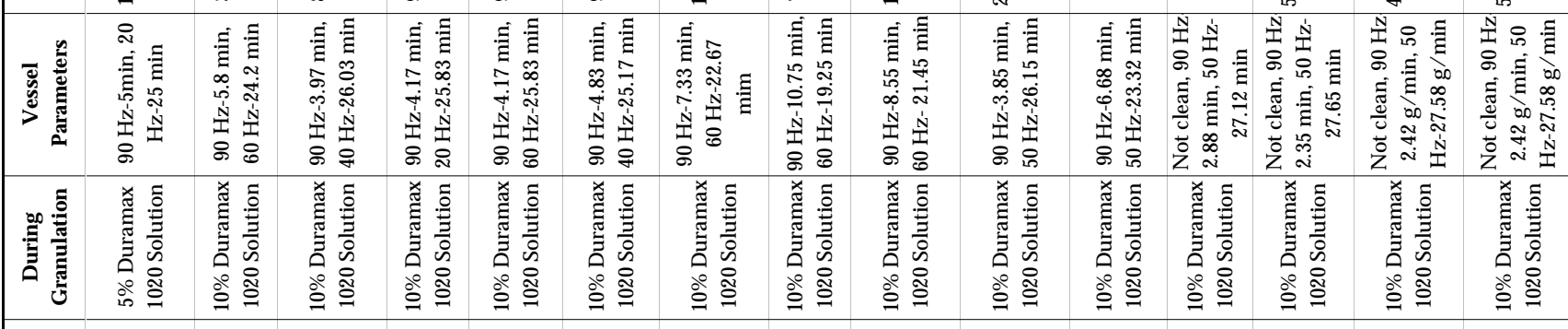

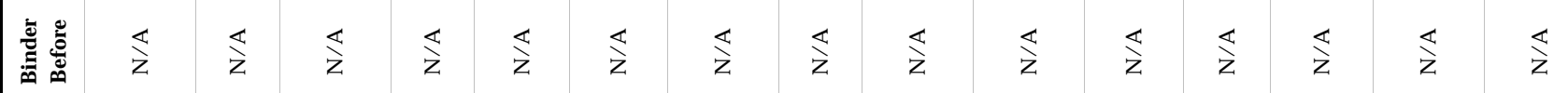

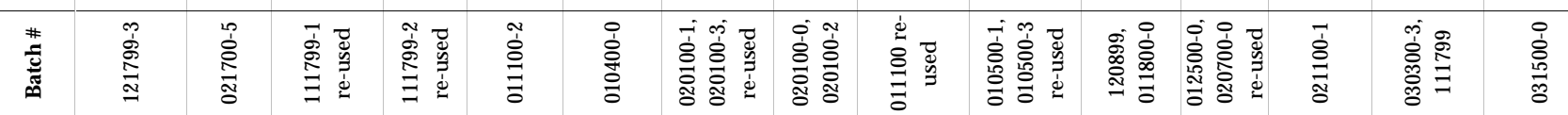

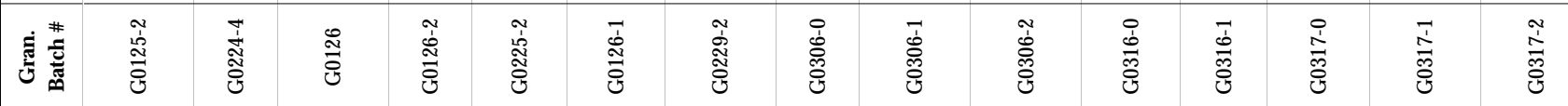

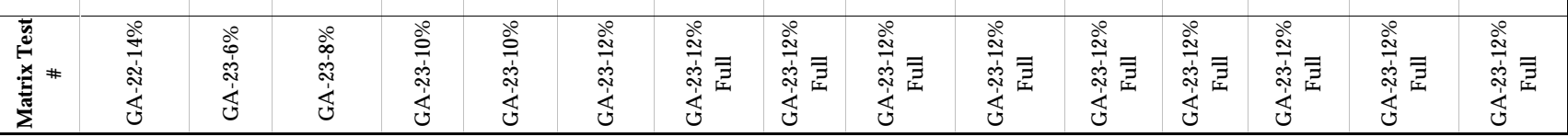




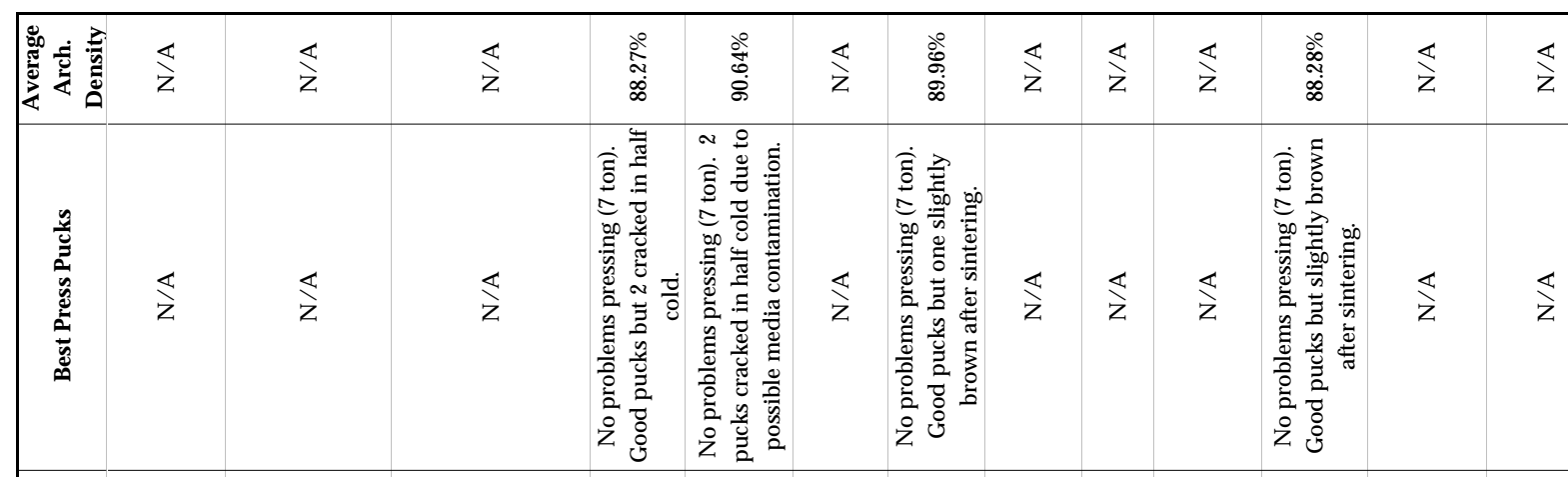

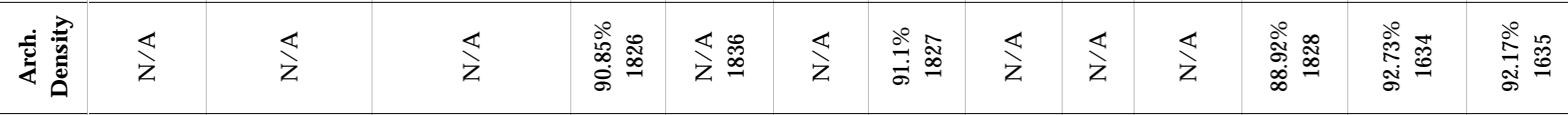

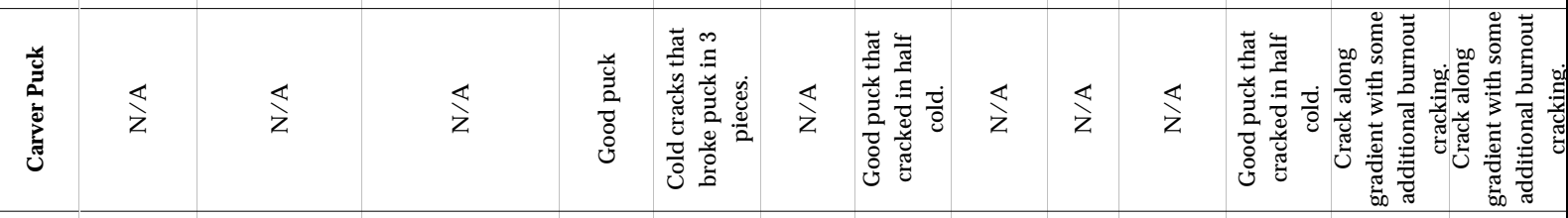

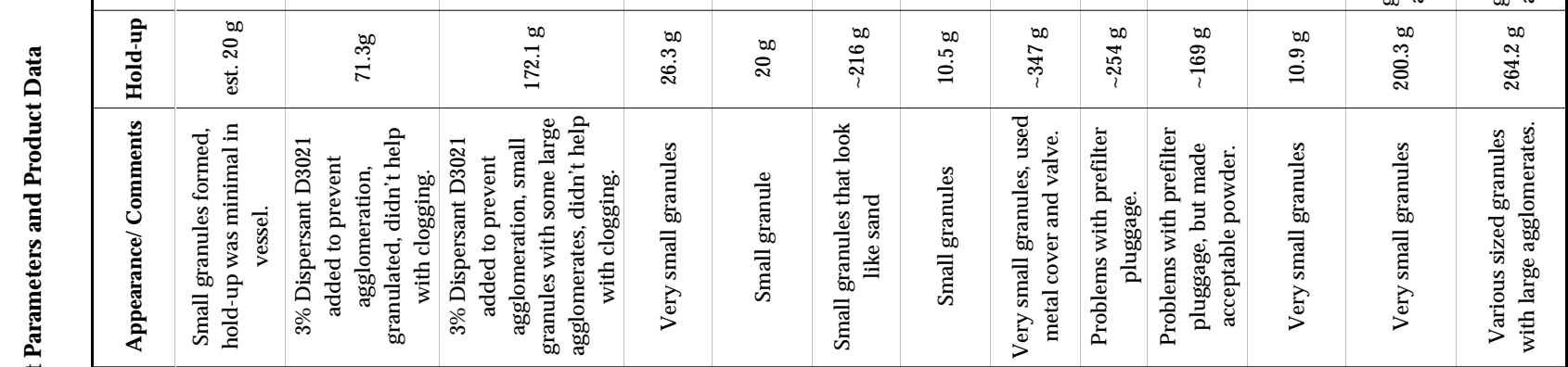

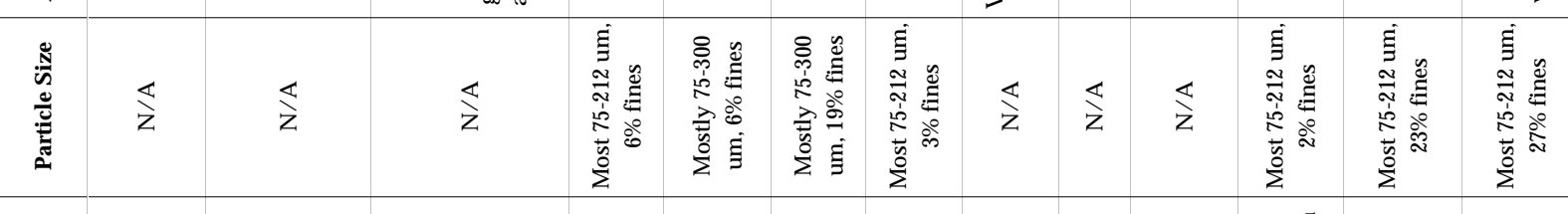

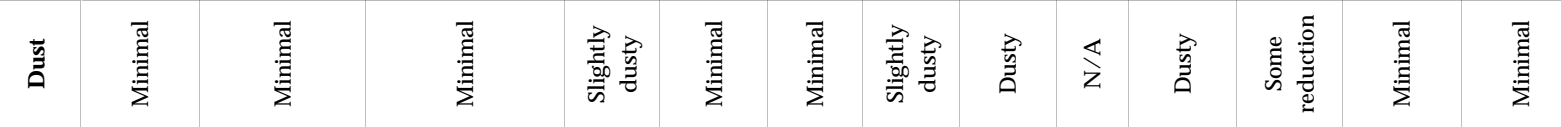

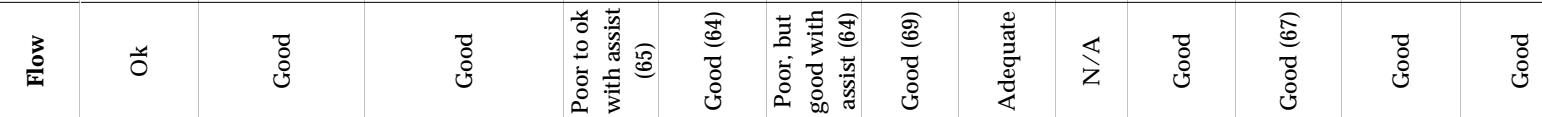
nI

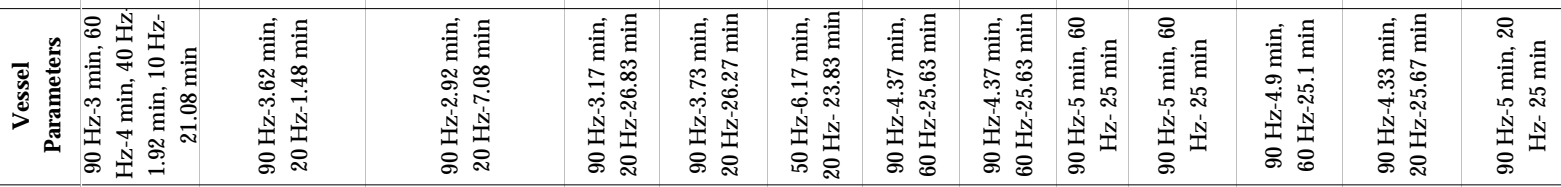

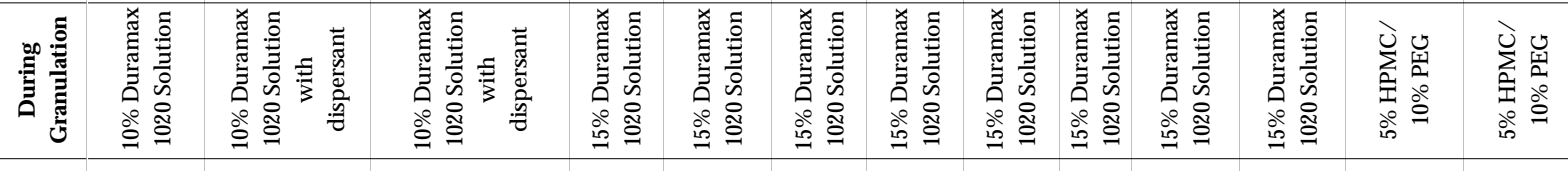

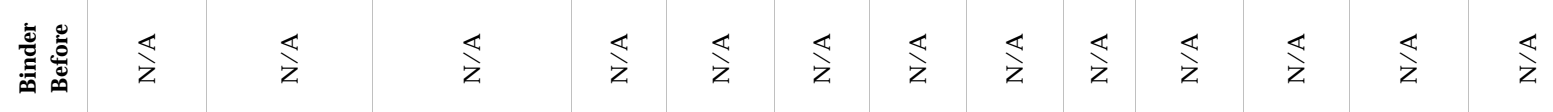

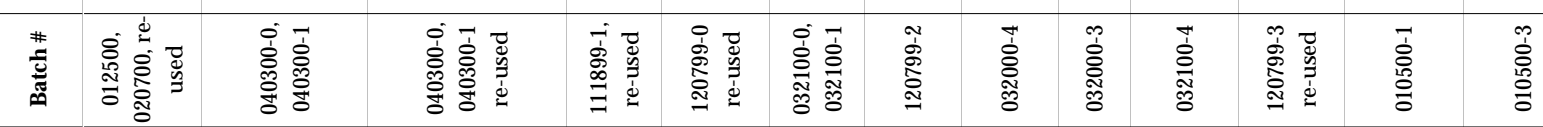

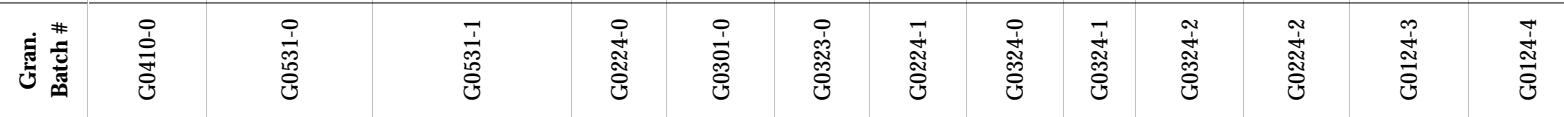

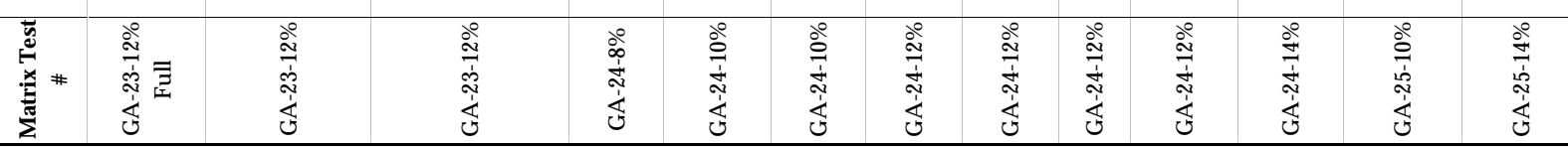




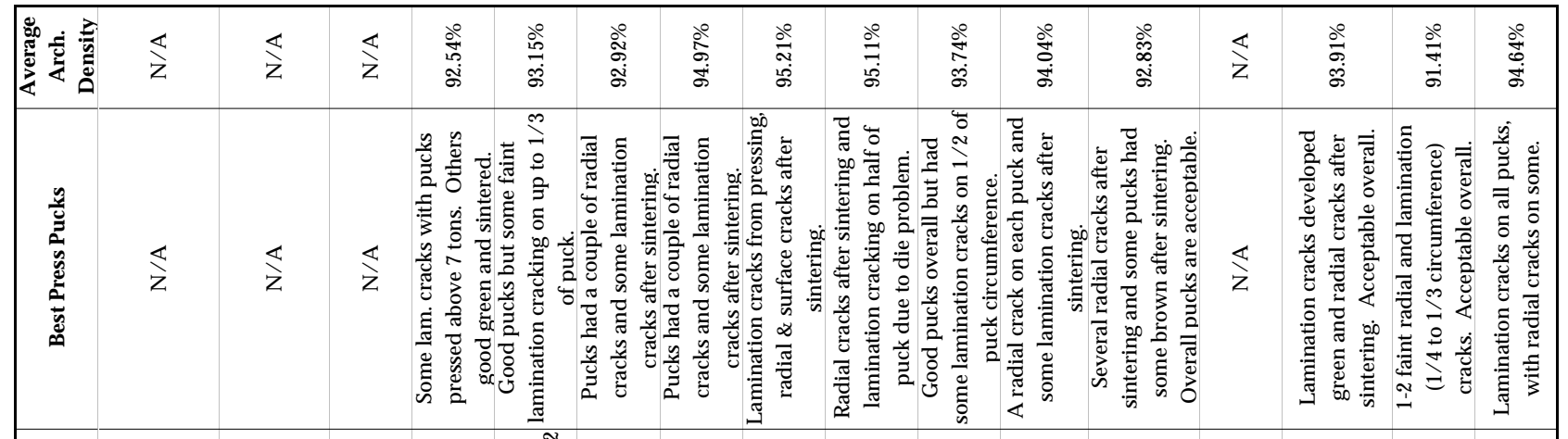

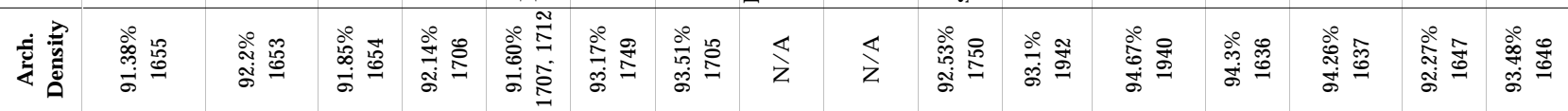

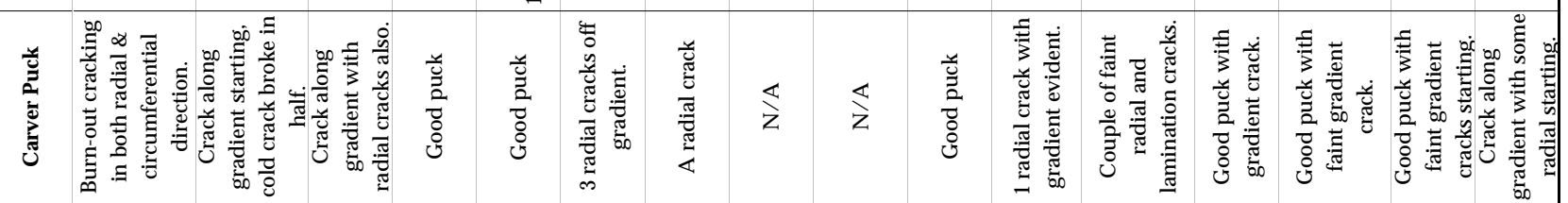

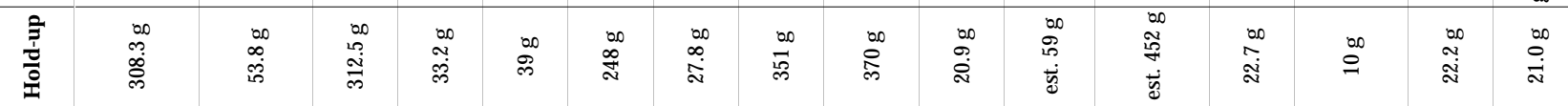

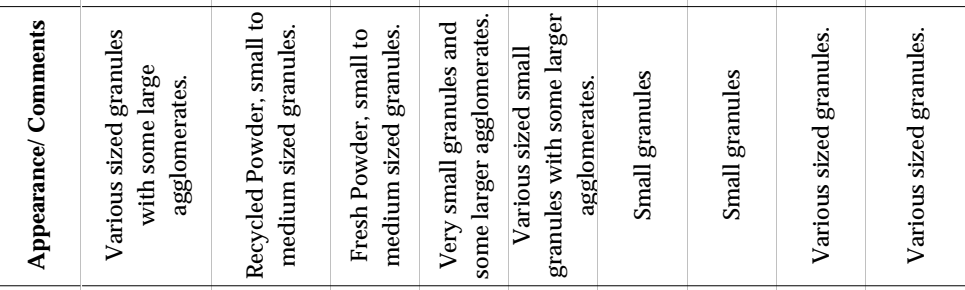

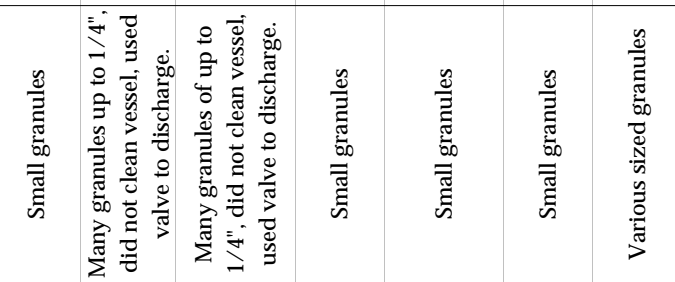

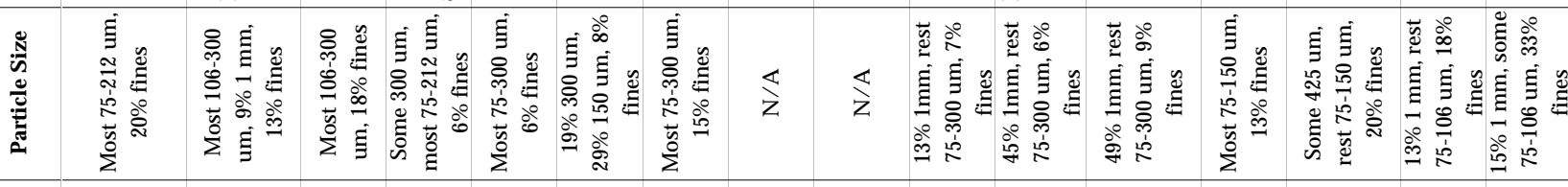

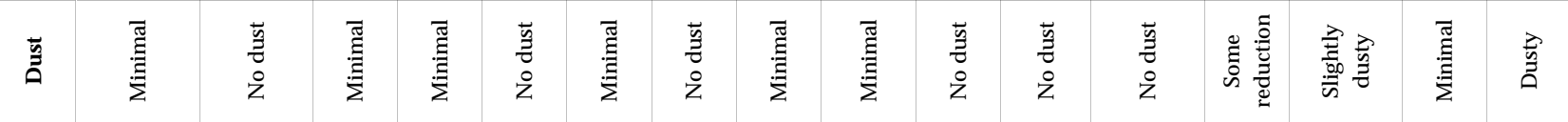

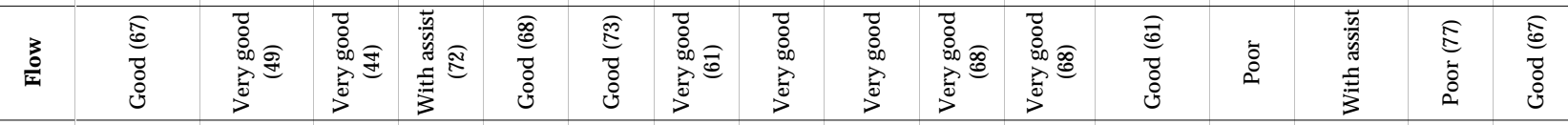
n

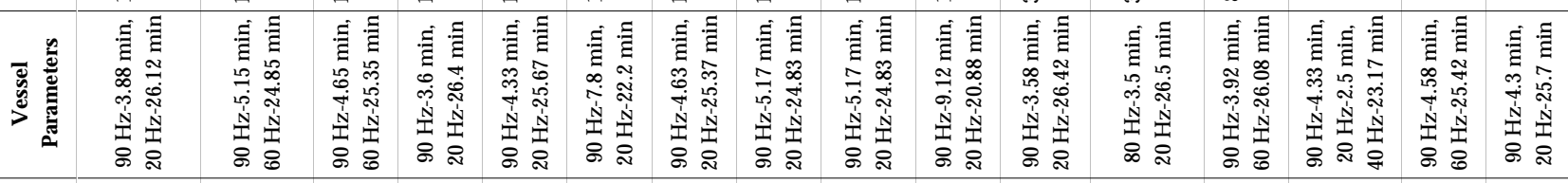

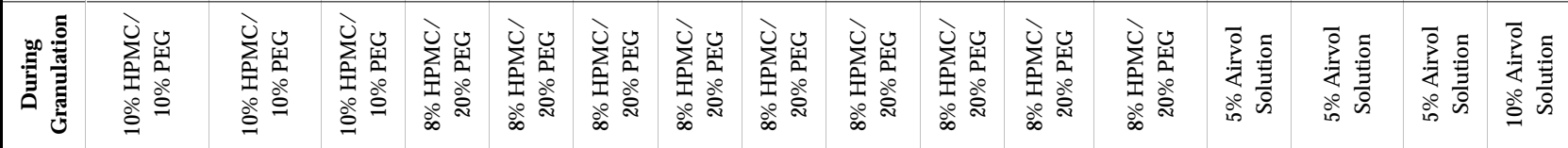

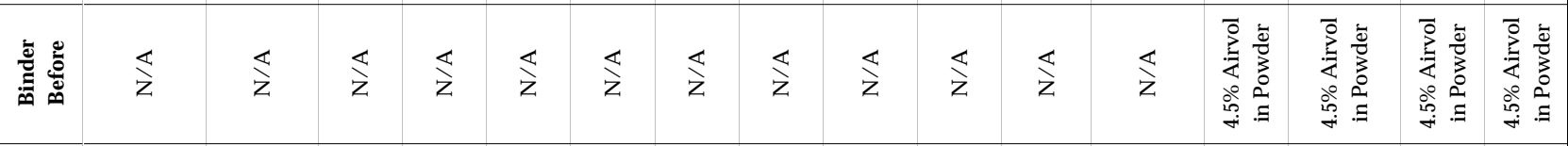

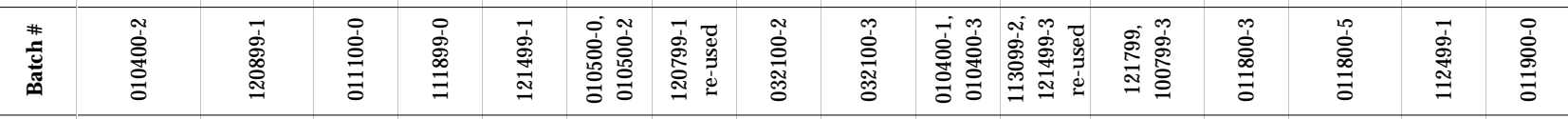

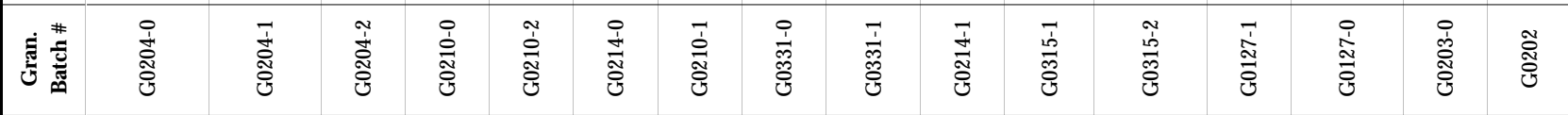

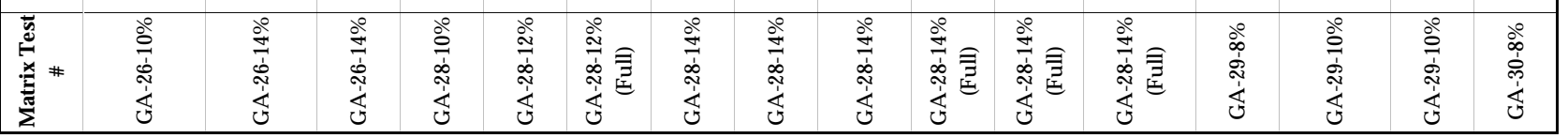




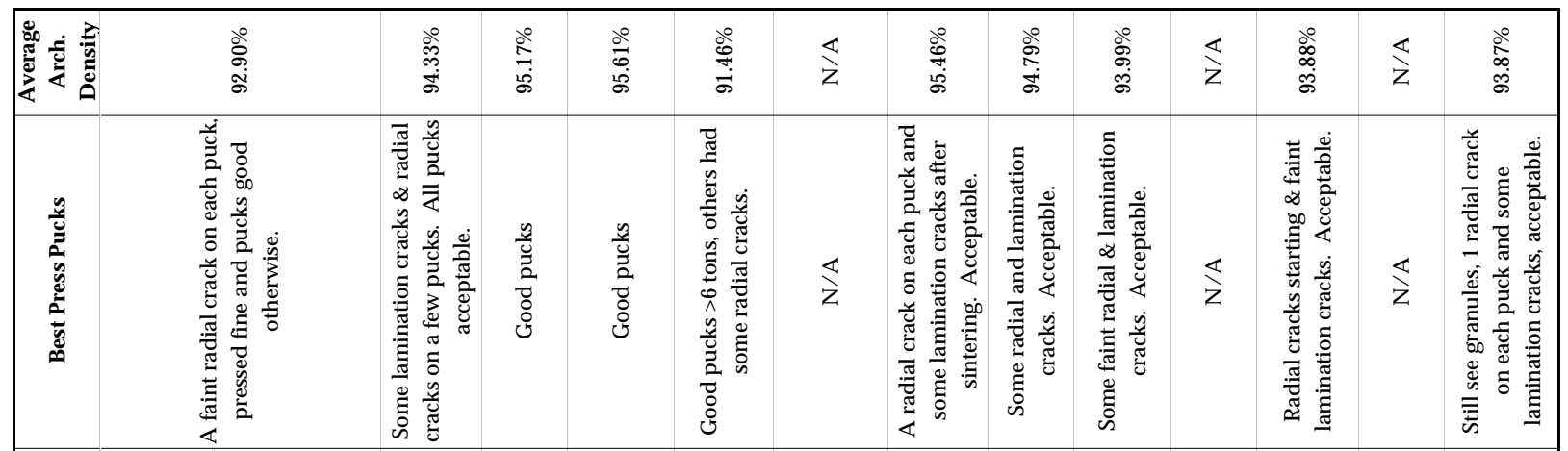

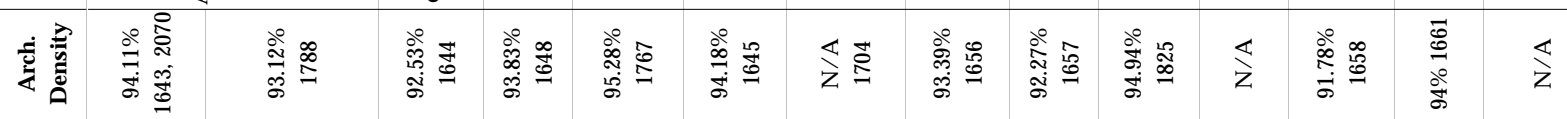

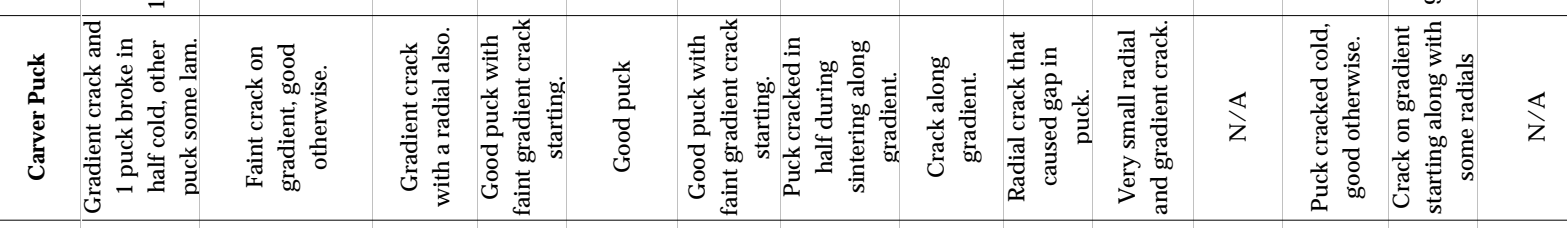

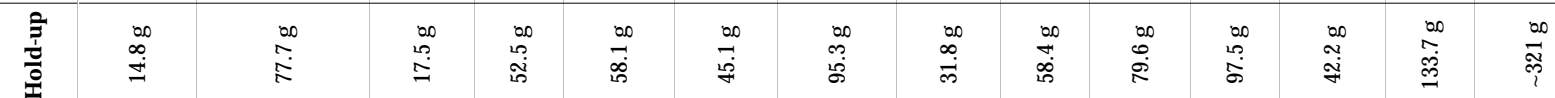

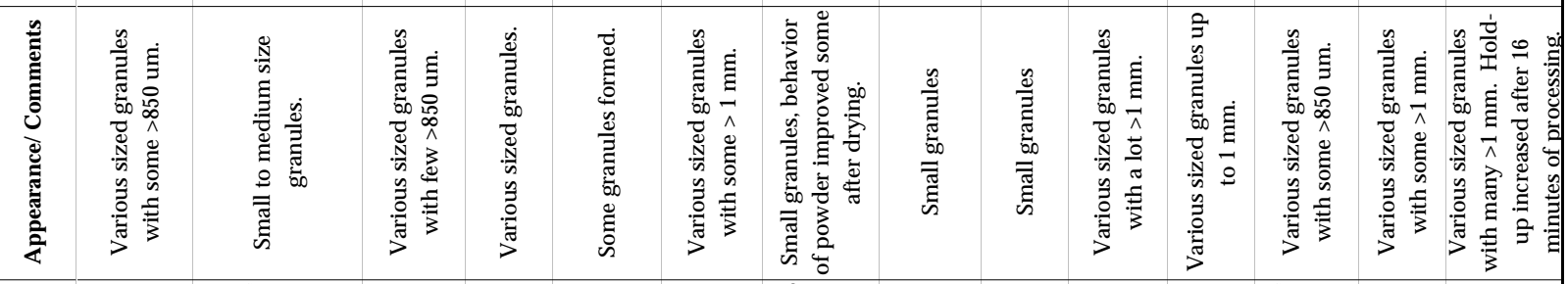

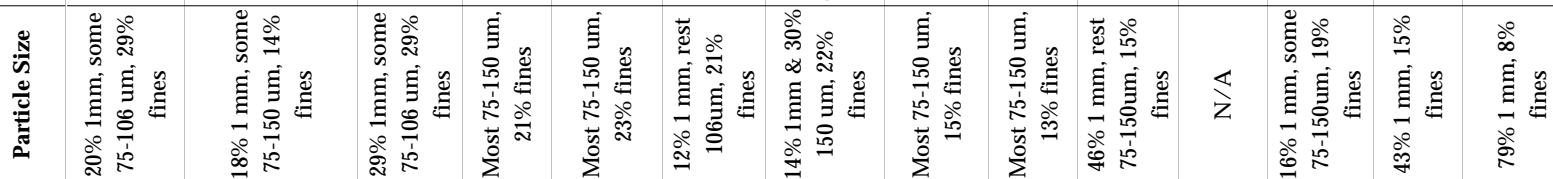

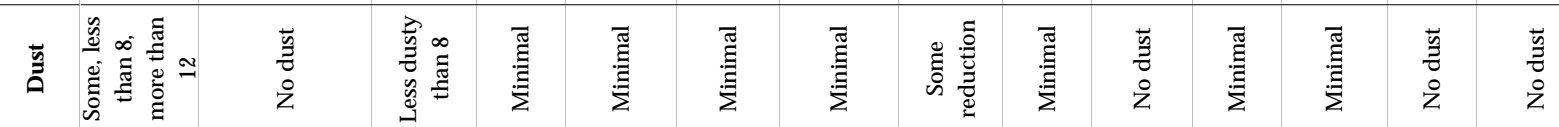

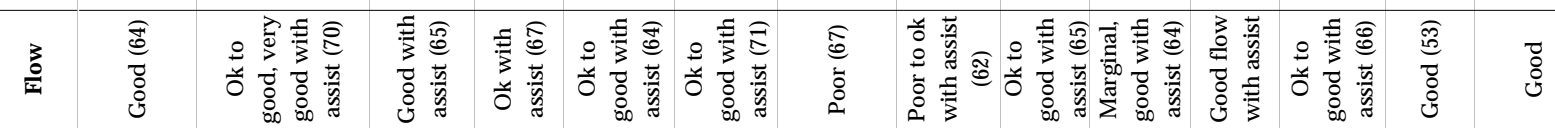

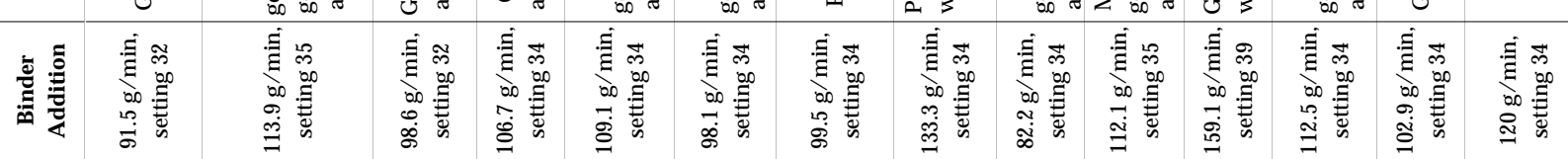

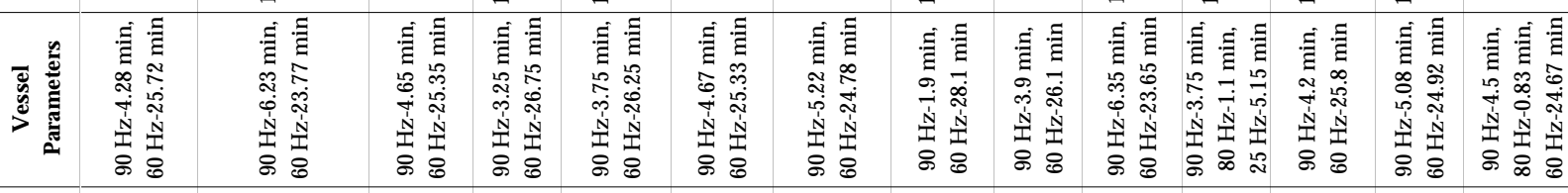

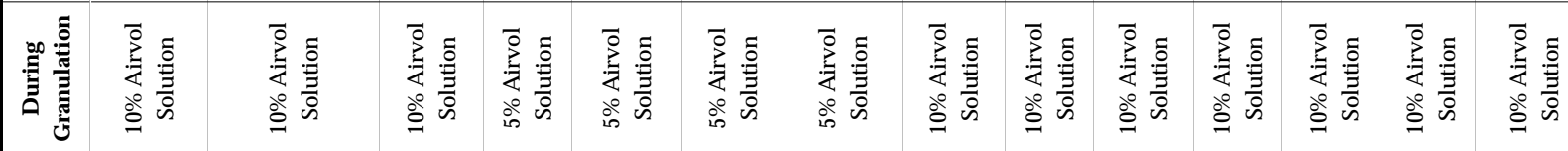

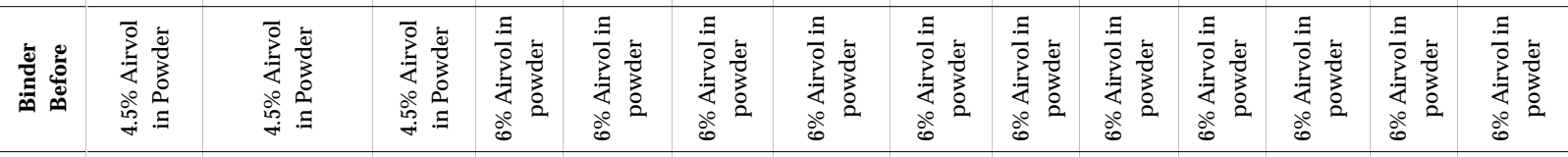

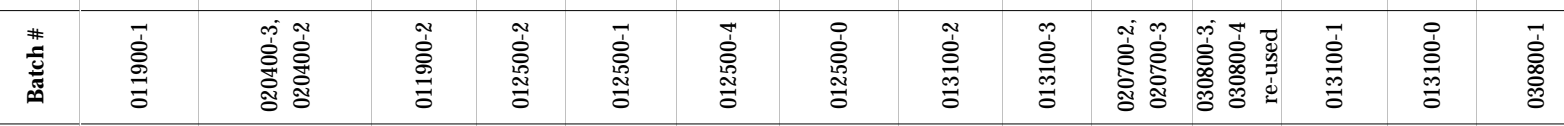

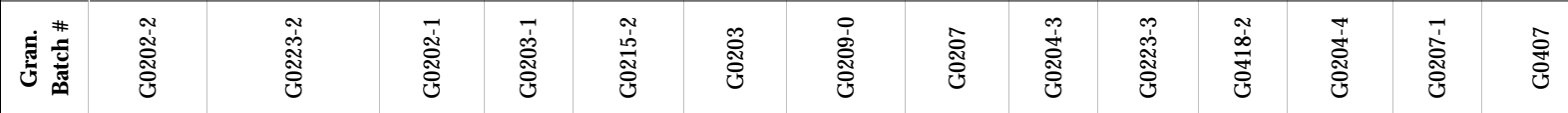

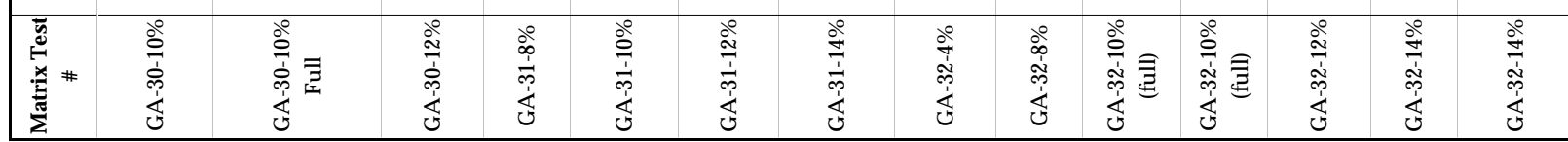




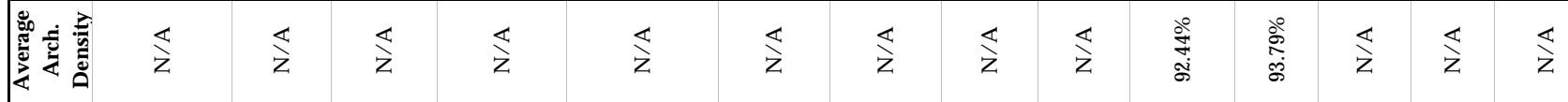

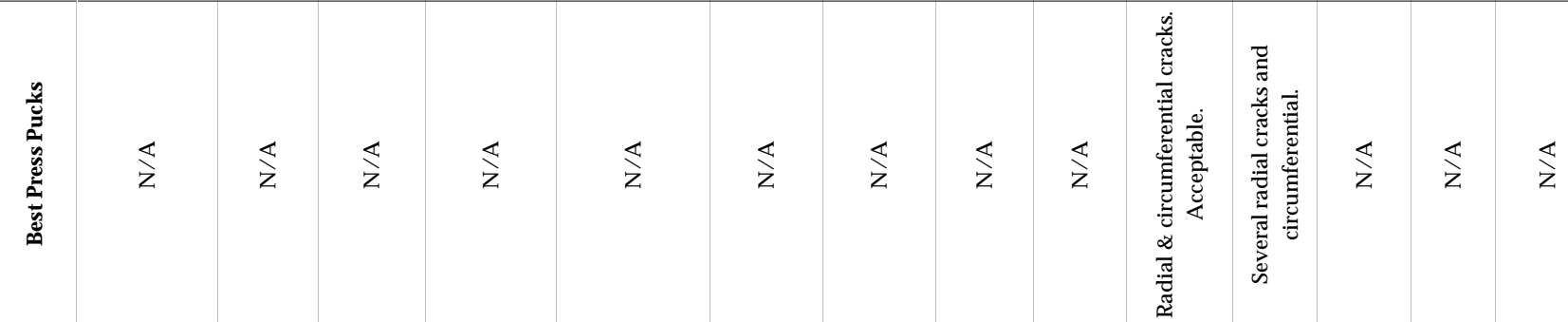

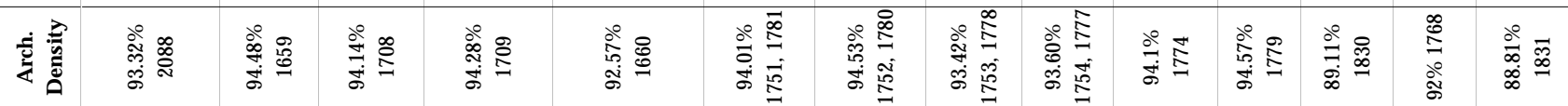

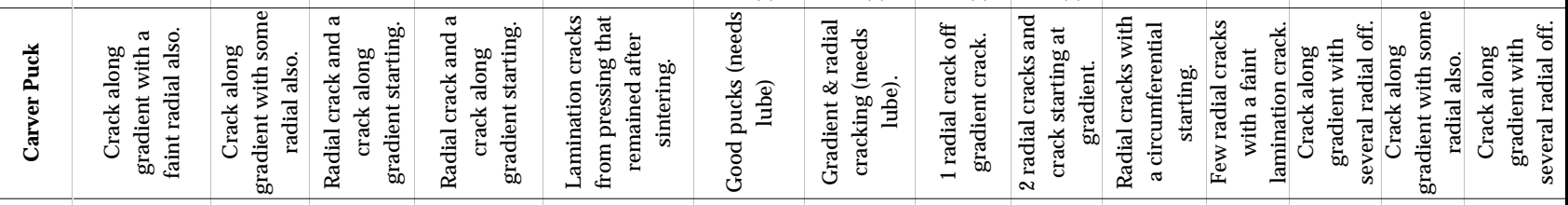

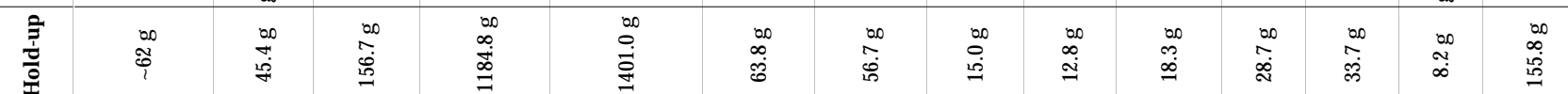

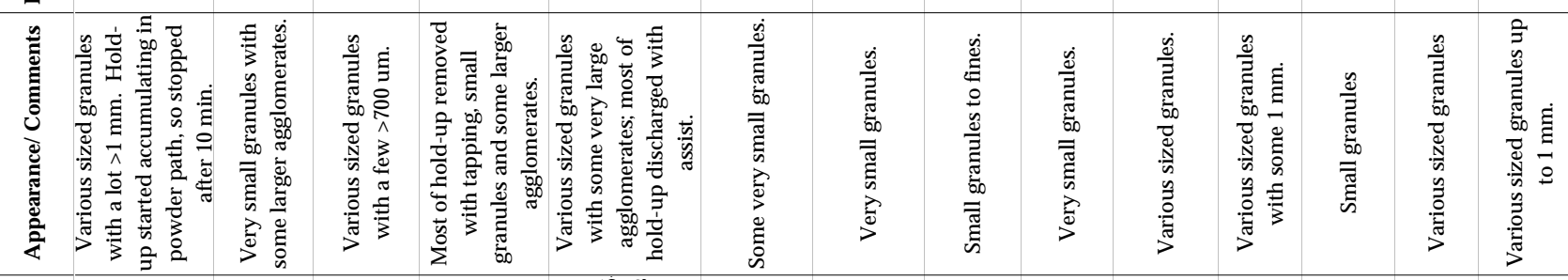

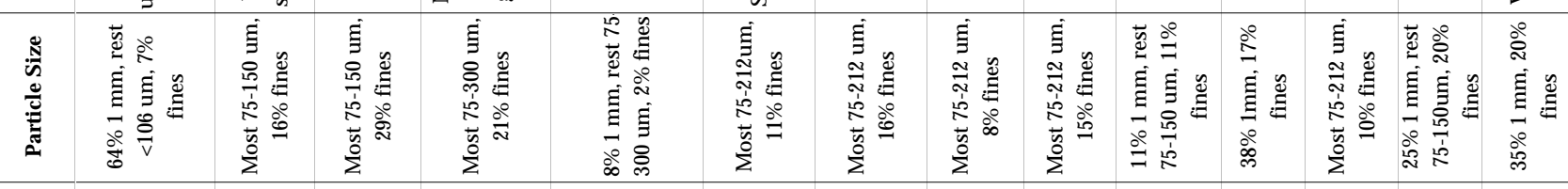

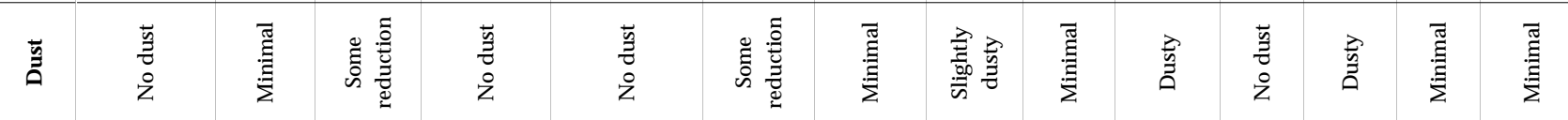

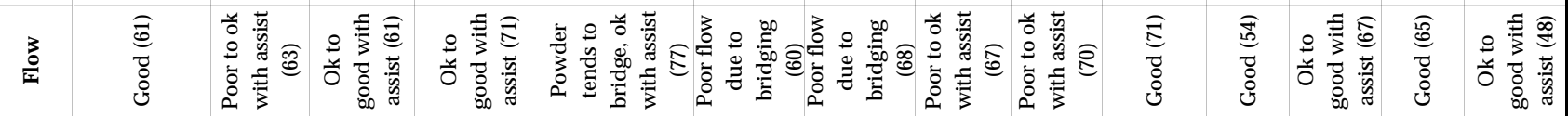

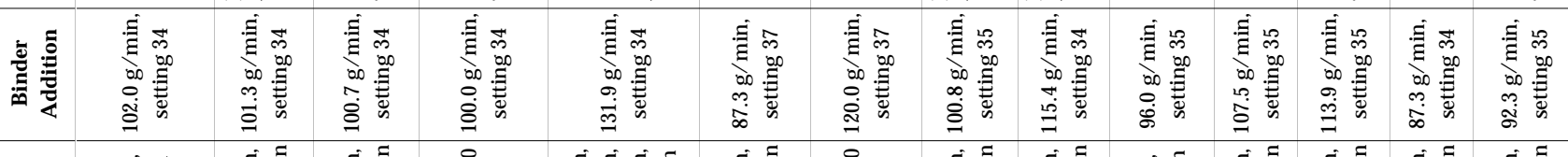

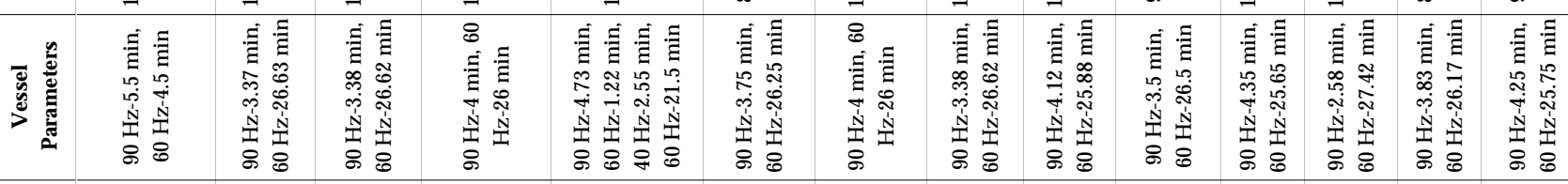

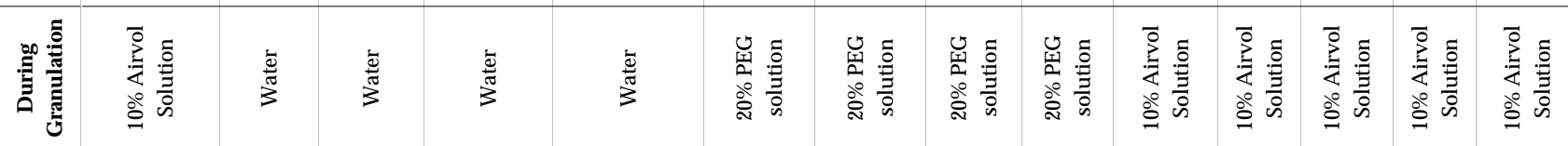

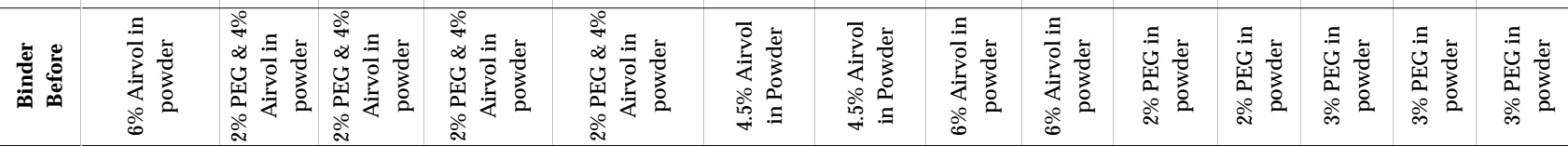

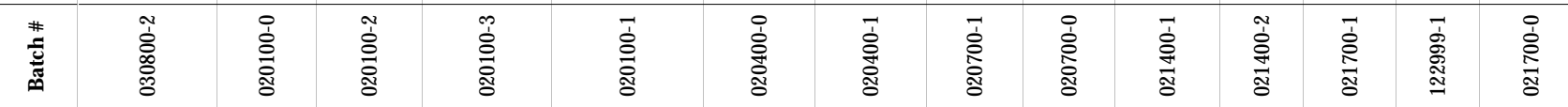

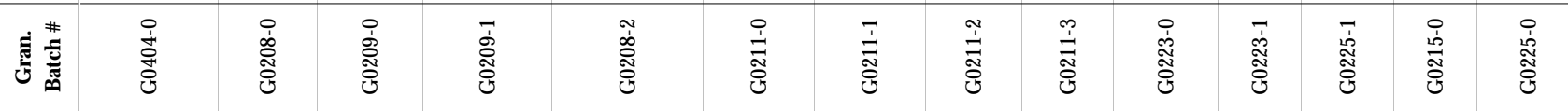

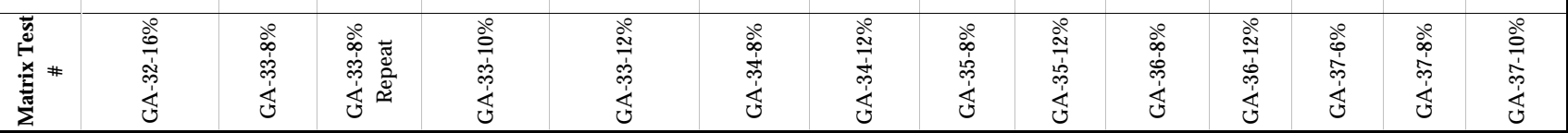




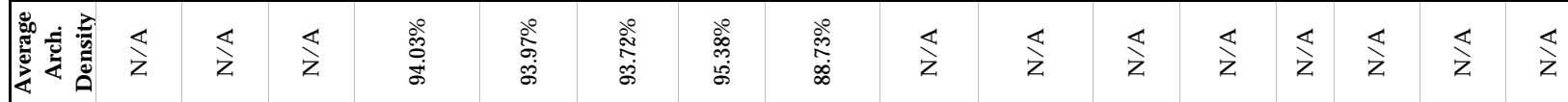

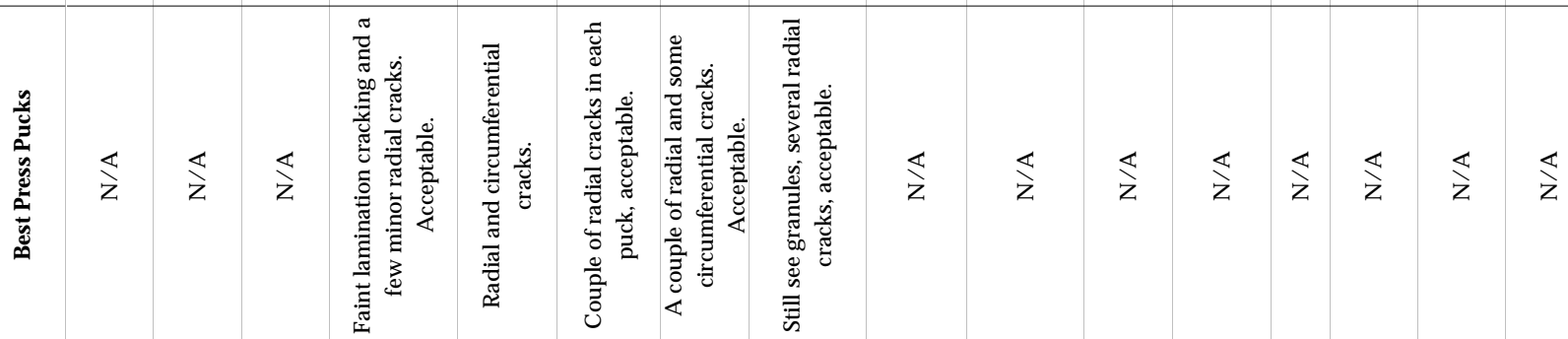

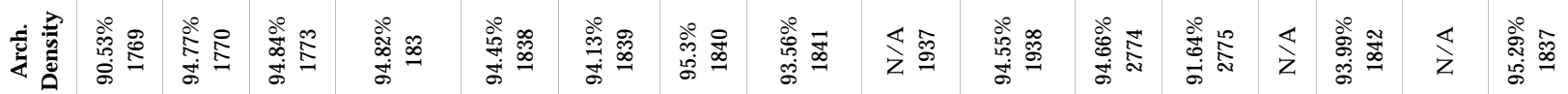

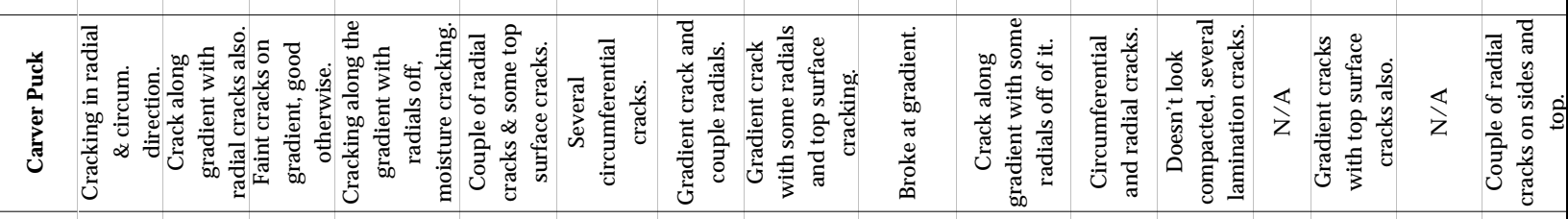

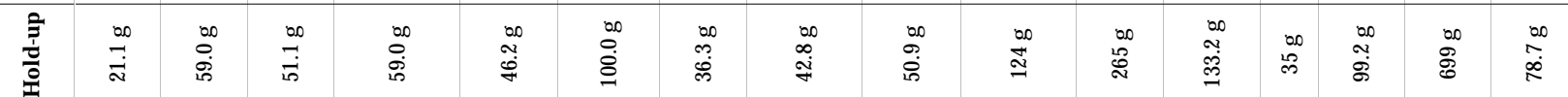

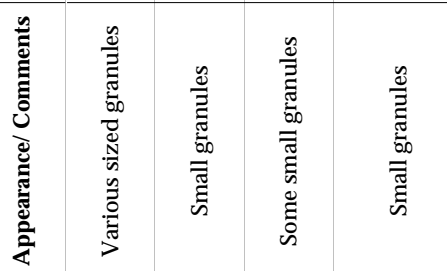

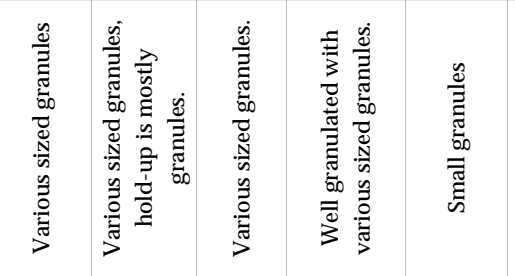

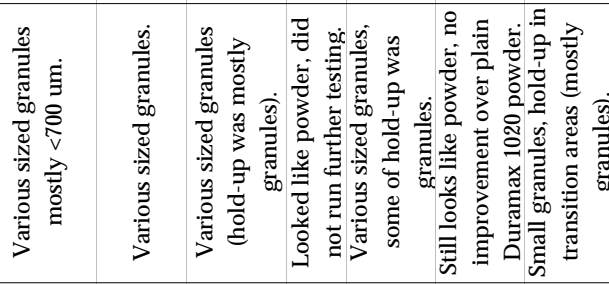

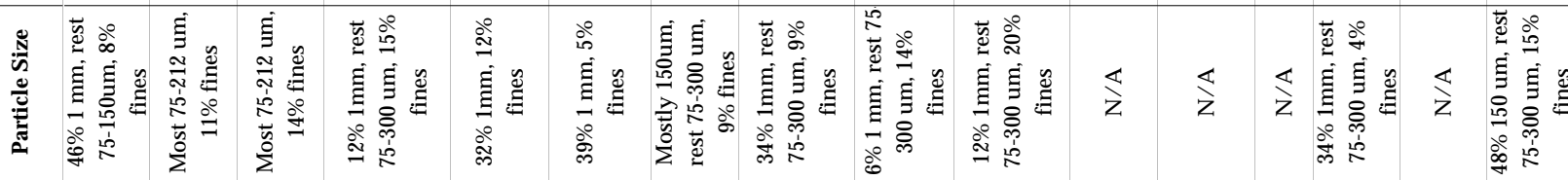

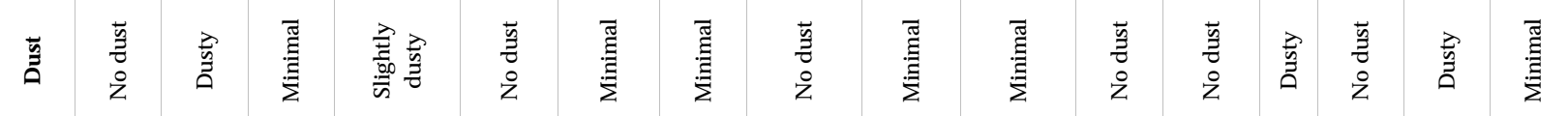

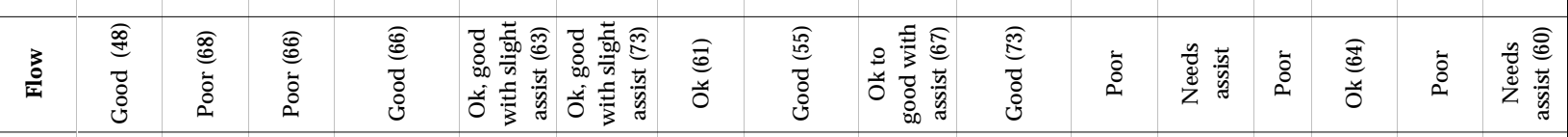

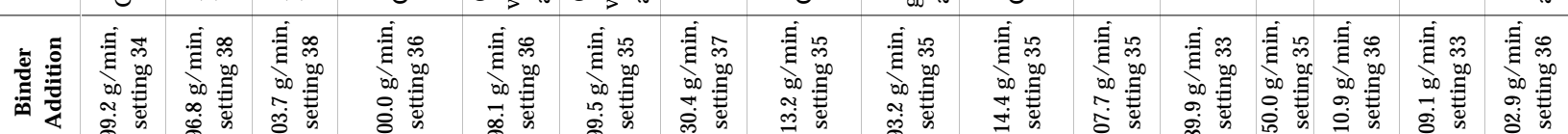

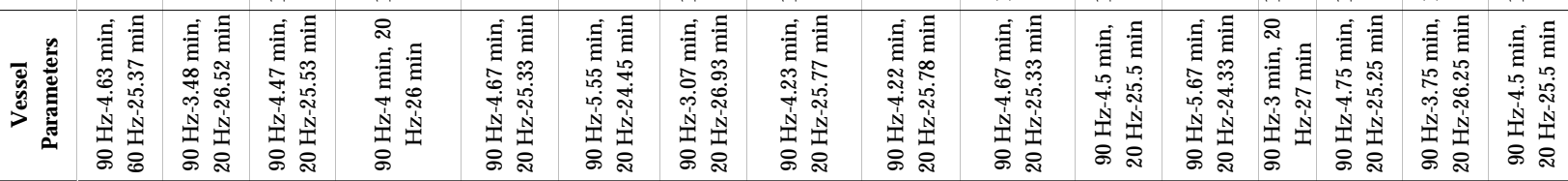

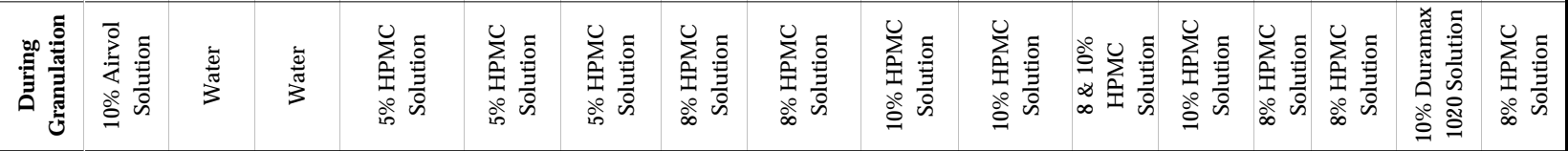

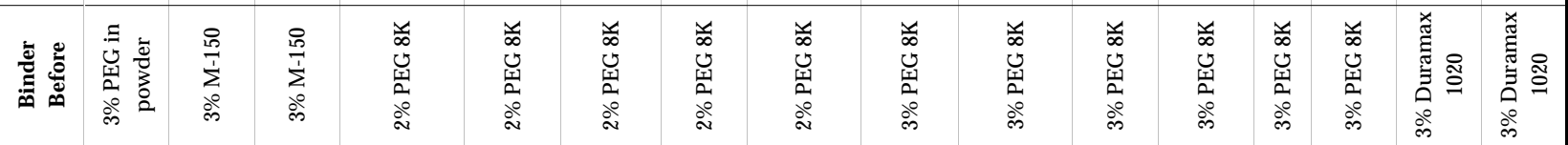

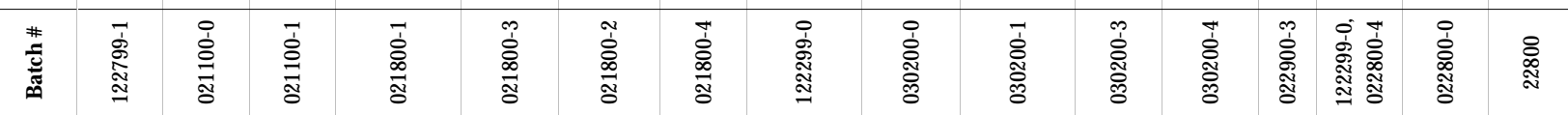

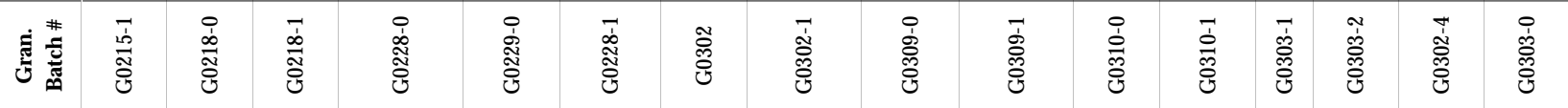

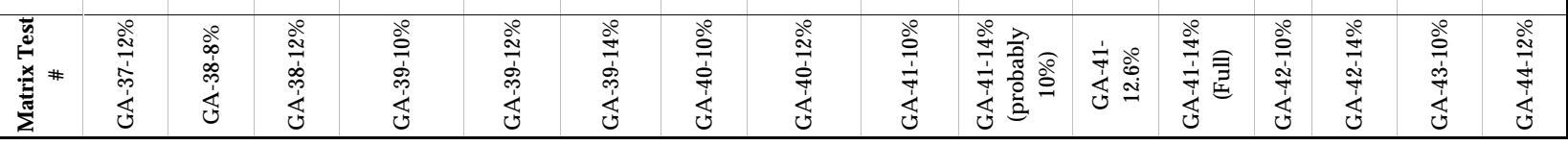




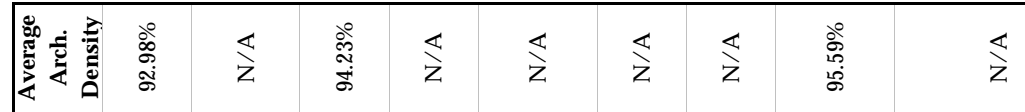

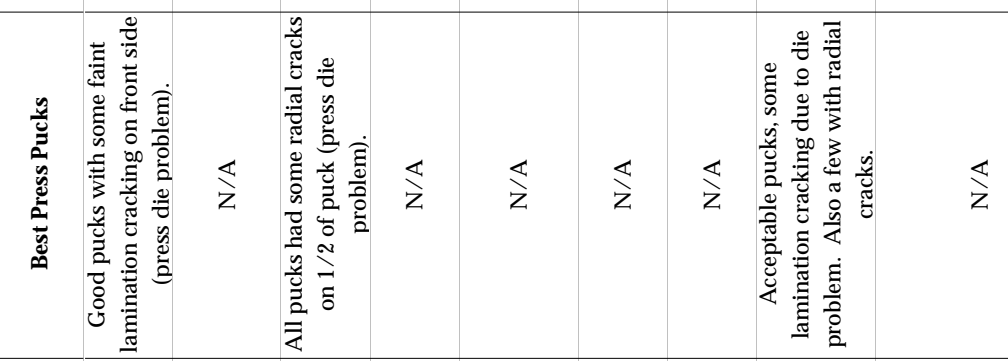

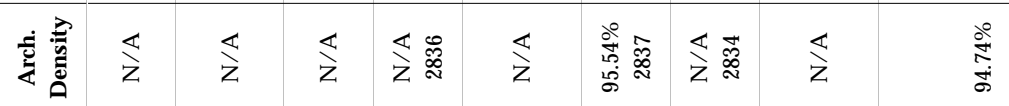

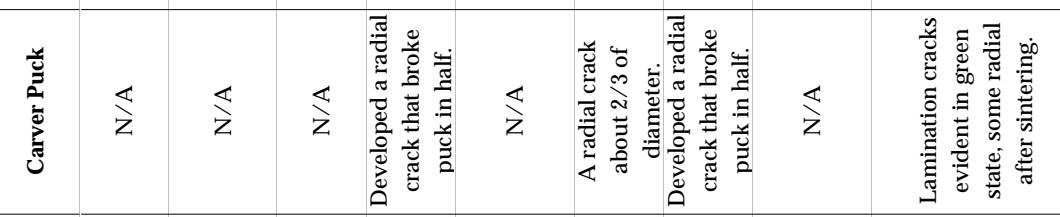

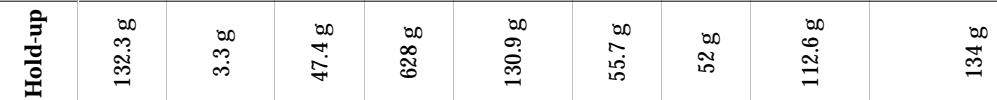

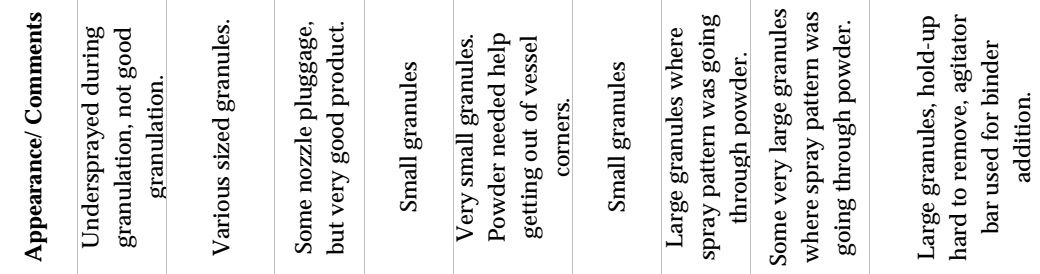

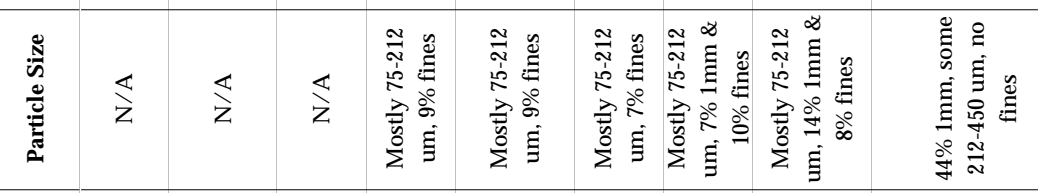

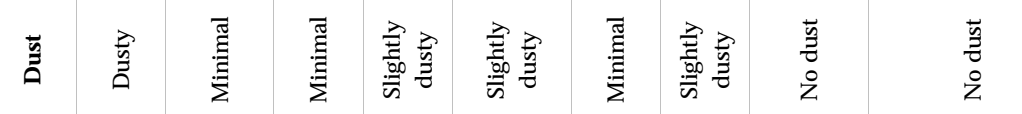

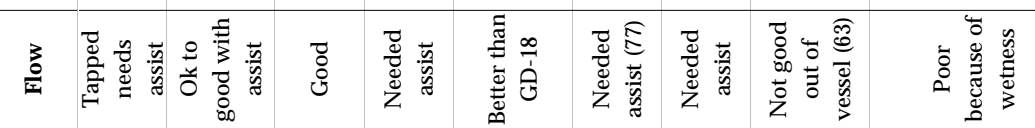

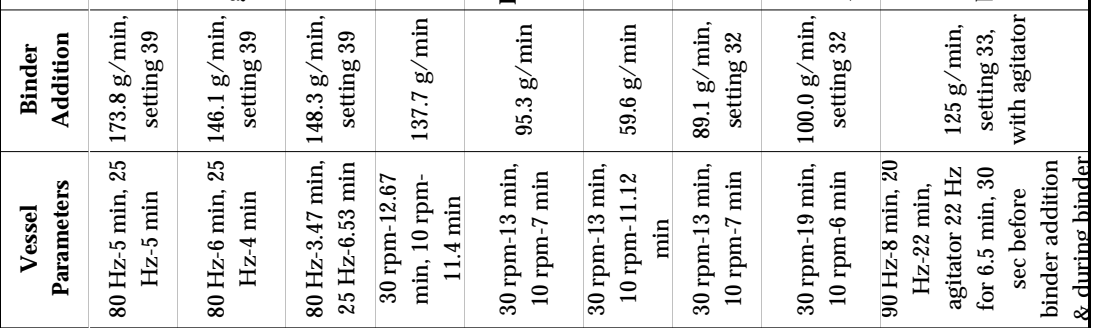

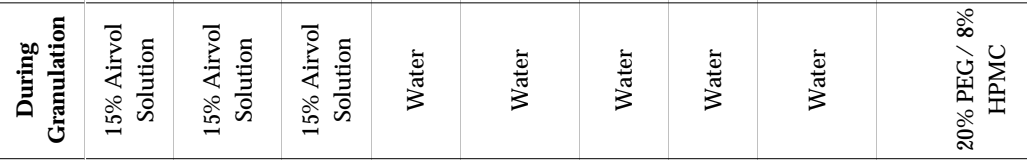

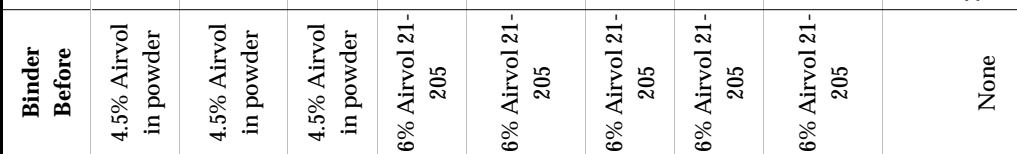

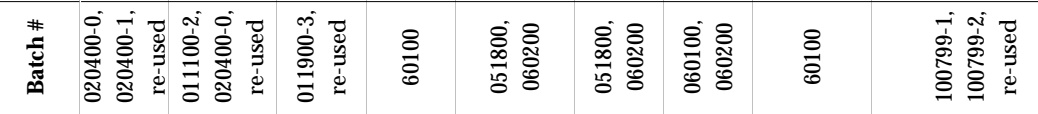

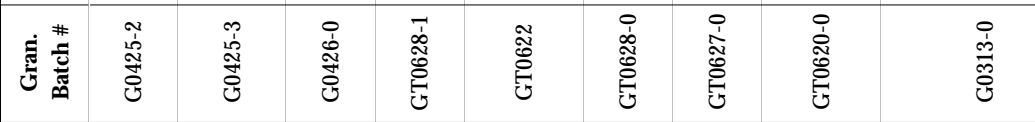

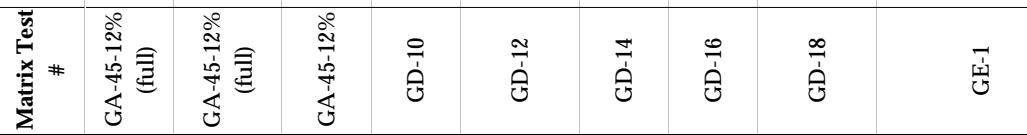


Appendix E - Summary of Uranium Granulation Test Parameters and Product Data

\begin{tabular}{|c|c|c|c|c|c|c|c|c|c|c|c|c|}
\hline Test \# & $\begin{array}{l}\text { Gran. } \\
\text { Batch \# }\end{array}$ & Batch \# & $\begin{array}{l}\text { Binder } \\
\text { Before }\end{array}$ & $\begin{array}{c}\text { During } \\
\text { Granulation }\end{array}$ & $\begin{array}{l}\text { Granulation } \\
\text { Parameters }\end{array}$ & $\begin{array}{c}\text { Binder } \\
\text { Addition }\end{array}$ & Flow & Dust & Appearance/ Comments & Hold-up & Carver Puck & $\begin{array}{c}\text { Arch. } \\
\text { Density }\end{array}$ \\
\hline GB-1-12\% & G0218-2 & 021100-3 & N/A & $\begin{array}{l}8 \% \text { HPMC / } \\
20 \% \text { PEG Sol'n }\end{array}$ & $\begin{array}{l}90 \mathrm{~Hz}-3.82 \mathrm{~min}, \\
20 \mathrm{~Hz}-25.18 \mathrm{~min}\end{array}$ & $112.1 \mathrm{~g} / \mathrm{min}$ & Ok & $\begin{array}{c}\text { Not too } \\
\text { dusty }\end{array}$ & $\begin{array}{c}\text { Small granules, but a lot of } \\
\text { hold-up. }\end{array}$ & $350.0 \mathrm{~g}$ & Some radial cracking. & 92.17 \\
\hline GB-1-14\% & GU0308-0 & 030300-2 & $\mathrm{N} / \mathrm{A}$ & $\begin{array}{l}\text { 8\% HPMC/ } \\
20 \% \text { PEG Sol'n }\end{array}$ & $\begin{array}{l}90 \mathrm{~Hz}-4.94 \mathrm{~min}, \\
20 \mathrm{~Hz}-25.06 \mathrm{~min}\end{array}$ & $104.1 \mathrm{~g} / \mathrm{min}$ & Good & $\begin{array}{l}\text { Some } \\
\text { dust }\end{array}$ & Very small granules & $489 \mathrm{~g}$ & $\begin{array}{l}\text { A couple of radial cracks, } \\
\text { some cracking on bottom. }\end{array}$ & 92.26 \\
\hline GB-2-12\% & G0228-2 & $021100-5$ & N/A & $\begin{array}{l}20 \% \text { PEG } \\
\text { Solution }\end{array}$ & $\begin{array}{c}90 \mathrm{~Hz}-5.7 \mathrm{~min}, 20 \\
\mathrm{~Hz}-24.3 \mathrm{~min}\end{array}$ & $92.7 \mathrm{~g} / \mathrm{min}$ & Ok for $U$ & Minimal & $\begin{array}{l}\text { Very small granules, all of } \\
\text { binder didn't go in. }\end{array}$ & $375 \mathrm{~g}$ & $\begin{array}{l}\text { A couple of radial cracks, } \\
\text { chattering when pressed. }\end{array}$ & 92.45 \\
\hline GB-3-10\% & G0229-3 & 021400-3 & $\begin{array}{l}8 \% \text { Airvol to } \\
\text { Precursors }\end{array}$ & Water & $\begin{array}{c}90 \mathrm{~Hz}-3.9 \mathrm{~min}, 60 \\
\mathrm{~Hz}-26.1 \mathrm{~min}\end{array}$ & $126.9 \mathrm{~g} / \mathrm{min}$ & Ok to good & None & $\begin{array}{l}\text { Very small granules (powder } \\
\text { like) with some } 3 / 4 " \\
\text { agglomerates, some problems } \\
\text { with binder pumping system. }\end{array}$ & $1060.4 \mathrm{~g}$ & $\begin{array}{l}\text { Good pucks except } 1 \text { radial } \\
\text { crack on each. }\end{array}$ & 93.36 \\
\hline GB-4-10\% & G0301-2 & $021400-5$ & $\begin{array}{l}8 \% \text { Airvol to } \\
\text { Precursors }\end{array}$ & $\begin{array}{l}10 \% \text { Airvol } \\
\text { Solution }\end{array}$ & $\begin{array}{l}90 \mathrm{~Hz}-1.43 \mathrm{~min}, \\
60 \mathrm{~Hz}-28.57 \mathrm{~min}\end{array}$ & $709.8 \mathrm{~g} / \mathrm{min}$ & Ok & $\begin{array}{l}\text { Not too } \\
\text { dusty }\end{array}$ & Some small granules. & $52 \mathrm{~g}$ & Good pucks & 92.74 \\
\hline GB-4-10\% & GU0705 & 031400-2 & $\begin{array}{l}8 \% \text { Airvol to } \\
\text { Precursors }\end{array}$ & $\begin{array}{l}10 \% \text { Airvol } \\
\text { Solution }\end{array}$ & $\begin{array}{l}90 \mathrm{~Hz}-5 \mathrm{~min}, 60 \\
\mathrm{~Hz}-10 \mathrm{~min}\end{array}$ & $76.5 \mathrm{~g} / \mathrm{min}$ & Good & Minimal & Well granulated & $42.4 \mathrm{~g}$ & $\begin{array}{l}\text { Faint crack along gradient, } \\
\text { good otherwise. }\end{array}$ & 93.14 \\
\hline GB-4-12\% & GU0412-0 & 031400-0 & $\begin{array}{l}8 \% \text { Airvol to } \\
\text { Precursors }\end{array}$ & $\begin{array}{l}10 \% \text { Airvol } \\
\text { Solution }\end{array}$ & $\begin{array}{l}90 \mathrm{~Hz}-5.85 \mathrm{~min} \\
60 \mathrm{~Hz}-24.15 \mathrm{~min}\end{array}$ & $67.4 \mathrm{~g} / \mathrm{min}$ & $\begin{array}{l}\text { Good with } \\
\text { assist }\end{array}$ & None & $\begin{array}{l}\text { Small to large granules, a lot of } \\
\text { hold-up. Powder seemed wet. }\end{array}$ & $198 \mathrm{~g}$ & $\begin{array}{l}\text { Lamination cracks upon } \\
\text { ejection, additional radial } \\
\text { and moisture cracking } \\
\text { after sintering. }\end{array}$ & 91.51 \\
\hline GB-5-12\% & G0307-0 & 030300-0 & N/A & $\begin{array}{l}10 \% \text { Duramax } \\
1020 \text { Solution }\end{array}$ & $\begin{array}{l}90 \mathrm{~Hz}-4.96 \mathrm{~min}, \\
20 \mathrm{~Hz}-25.04 \mathrm{~min}\end{array}$ & $86.7 \mathrm{~g} / \mathrm{min}$ & Good & Minimal & $\begin{array}{c}\text { Small granules, but a lot of } \\
\text { hold-up. }\end{array}$ & $367 \mathrm{~g}$ & $\begin{array}{l}\text { Faint circumferential } \\
\text { cracking with a radial } \\
\text { crack also. }\end{array}$ & 92.23 \\
\hline GB-6-10\% & GU0309 & $030300-3$ & $\begin{array}{l}4 \% \text { PEG to } \\
\text { Precursors }\end{array}$ & $\begin{array}{l}10 \% \text { Airvol } \\
\text { Solution }\end{array}$ & $\begin{array}{l}90 \mathrm{~Hz}-5.85 \mathrm{~min}, \\
60 \mathrm{~Hz}-24.15 \mathrm{~min}\end{array}$ & $64.1 \mathrm{~g} / \mathrm{min}$ & Very good & No dust & $\begin{array}{c}\text { Various sized granules up to } \\
\qquad 1 / 4^{\prime \prime}\end{array}$ & $656.7 \mathrm{~g}$ & $\begin{array}{l}1 \text { puck broke, others had } \\
\text { cracks along gradient with } \\
\text { few radial cracks. }\end{array}$ & 91.26 \\
\hline GB-7-12\% & GU0315-0 & $0300-5$ & $\begin{array}{l}4 \% \text { PEG to } \\
\text { Precursors }\end{array}$ & $\begin{array}{l}8 \% \text { HPMC } \\
\text { Solution }\end{array}$ & $\begin{array}{c}90 \mathrm{~Hz}-4.8 \mathrm{~min}, 20 \\
\mathrm{~Hz}-25.2 \mathrm{~min}\end{array}$ & $122.4 \mathrm{~g} / \mathrm{min}$ & $\begin{array}{l}\text { Good with } \\
\text { assist }\end{array}$ & No dust & Various sized granules & $336.7 \mathrm{~g}$ & $\begin{array}{l}\text { All cracked during } \\
\text { pressing }\end{array}$ & $\mathrm{N} / \mathrm{A}$ \\
\hline GB-8-0\% & $\mathrm{N} / \mathrm{A}$ & 030900-0 & $\begin{array}{l}6.8 \% \\
\text { Duramax } \\
1020 \text { to } \\
\text { Precursors }\end{array}$ & $\mathrm{N} / \mathrm{A}$ & N/A & N/A & Poor & Dusty & Powder & $\mathrm{N} / \mathrm{A}$ & $\begin{array}{l}\text { Lamination cracks upon } \\
\text { ejection, additional radial } \\
\text { cracking after sintering. }\end{array}$ & 94.01 \\
\hline GB-8-10\% & GU0315-1 & $\begin{array}{l}\text { 030900-0, } \\
030900-1\end{array}$ & $\begin{array}{l}6.8 \% \\
\text { Duramax } \\
1020 \text { to } \\
\text { Precursors }\end{array}$ & Water & $\begin{array}{c}90 \mathrm{~Hz}-4.7 \mathrm{~min}, 20 \\
\mathrm{~Hz}-25.3 \mathrm{fin}\end{array}$ & $83.8 \mathrm{~g} / \mathrm{min}$ & $\begin{array}{l}\text { Needs } \\
\text { assist, better } \\
\text { than Ce }\end{array}$ & Dusty & $\begin{array}{l}\text { Looks like powder with very } \\
\text { small granules. }\end{array}$ & $224.2 \mathrm{~g}$ & $\begin{array}{l}\text { A couple of faint radial and } \\
\text { circumferential cracks. }\end{array}$ & 93.41 \\
\hline GB-8-12\% & G0321-0 & 30900 & $\begin{array}{l}6.8 \% \\
\text { Duramax } \\
1020 \text { to } \\
\text { Precursors }\end{array}$ & Water & $\begin{array}{l}90 \mathrm{~Hz}-4.96 \mathrm{~min}, \\
20 \mathrm{~Hz}-25.04 \mathrm{~min}\end{array}$ & $93.1 \mathrm{~g} / \mathrm{min}$ & Good & No dust & $\begin{array}{l}\text { Small granules, but a lot of } \\
\text { hold-up. Powder is acceptble, } \\
\text { but process is not. }\end{array}$ & $1088.2 \mathrm{~g}$ & N/A & $\mathrm{N} / \mathrm{A}$ \\
\hline GB-9-12\% & GU0323-0 & 030900-3 & $\begin{array}{c}6.8 \% \\
\text { Duramax } \\
1020 \text { to } \\
\text { Precursors }\end{array}$ & $\begin{array}{l}10 \% \text { HPMC } \\
\text { Solution }\end{array}$ & $\begin{array}{c}80 \mathrm{~Hz}-4.5 \mathrm{~min}, 20 \\
\mathrm{~Hz}-25.5 \mathrm{~min}\end{array}$ & $102.9 \mathrm{~g} / \mathrm{min}$ & Ok & No dust & $\begin{array}{c}\text { Clumps of granules, hold-up is } \\
\text { granules. }\end{array}$ & $\sim 1 \mathrm{~kg}$ & $\begin{array}{l}\text { Some sticking to top } \\
\text { punch, which developed } \\
\text { radial cracks in sintered } \\
\text { pucks. Good otherwise. }\end{array}$ & 91.77 \\
\hline
\end{tabular}


Appendix F - Puck Data for Granulation Matrix Testing

\begin{tabular}{|c|c|c|c|c|c|c|c|c|c|c|c|c|c|c|c|c|c|c|}
\hline Batch ID & $\begin{array}{c}\text { Puck } \\
\text { ID }\end{array}$ & $\begin{array}{c}\text { Green } \\
\text { Puck } \\
\text { Diam. } \\
\text { (in.) }\end{array}$ & $\begin{array}{c}\text { Sintered } \\
\text { Puck } \\
\text { Diameter } \\
\text { (in.) }\end{array}$ & $\begin{array}{c}\text { Green } \\
\text { Weight } \\
\text { (g) }\end{array}$ & \begin{tabular}{|c|} 
Sintered \\
Puck \\
Weight \\
(g)
\end{tabular} & $\begin{array}{c}\text { Puck } \\
\text { Suspended } \\
\text { Weight (g) }\end{array}$ & $\begin{array}{c}\text { Wet } \\
\text { Weight } \\
\text { (g) }\end{array}$ & $\begin{array}{c}\text { Green } \\
\text { Puck } \\
\text { Thick. } \\
\text { (in.) }\end{array}$ & $\begin{array}{c}\text { Sintered } \\
\text { Puck } \\
\text { Thick. } \\
\text { (in.) }\end{array}$ & $\begin{array}{c}\text { Green } \\
\text { Density } \\
\text { (g/cc) }\end{array}$ & $\begin{array}{c}\text { Archi- } \\
\text { medes } \\
\text { Density } \\
\text { (g/cc) }\end{array}$ & $\begin{array}{c}\text { Archi- } \\
\text { medes } \\
\text { Density } \\
\text { (\%TD) }\end{array}$ & $\begin{array}{c}\text { Geom. } \\
\text { Density } \\
\text { (g/cc) }\end{array}$ & $\begin{array}{l}\text { Geom. } \\
\text { (\%TD) }\end{array}$ & $\begin{array}{c}\text { Mass \% } \\
\text { Shrink- } \\
\text { age }\end{array}$ & $\begin{array}{c}\text { Volume } \\
\% \\
\text { Shinkage }\end{array}$ & \begin{tabular}{|c|} 
Diam. \\
$\%$ \\
Shrink- \\
age
\end{tabular} & $\begin{array}{l}\text { Powder } \\
\% \text { Moist }\end{array}$ \\
\hline GA-1-0\% & 1520 & 3.256 & 2.600 & 463.7 & 442.8 & 352.515 & 442.8 & 1.390 & N/A & 2.44 & 4.90 & 94.32 & $\mathrm{~N} / \mathrm{A}$ & $\mathrm{N} / \mathrm{A}$ & 4.51 & N/A & 20.15 & 0.7 \\
\hline GA-1-0\% & 1775 & 3.256 & 2.575 & 499.7 & 472.5 & 375.762 & 469.8 & 1.424 & 1.140 & 2.57 & 5.02 & 96.63 & 4.86 & 93.37 & 5.44 & 49.92 & 20.92 & 0.4 \\
\hline GA-1-4\% & 1505 & 3.254 & 2.621 & 451.8 & 422.2 & 335.784 & 422.4 & 1.228 & 0.985 & 2.70 & 4.87 & 93.74 & 4.85 & 93.20 & 6.55 & 47.95 & 19.45 & 4.3 \\
\hline GA-1-8\% & 1500 & 3.254 & N/A & 454.6 & N/A & N/A & N/A & 1.203 & N/A & 2.77 & $\mathrm{~N} / \mathrm{A}$ & N/A & $\mathrm{N} / \mathrm{A}$ & N/A & N/A & N/A & N/A & 8.0 \\
\hline GA-1-12\%, CP & 1499 & 3.254 & N/A & 451.9 & N/A & N/A & N/A & 1.171 & $\mathrm{~N} / \mathrm{A}$ & 2.83 & $\mathrm{~N} / \mathrm{A}$ & N/A & $\mathrm{N} / \mathrm{A}$ & $\mathrm{N} / \mathrm{A}$ & $\mathrm{N} / \mathrm{A}$ & $\mathrm{N} / \mathrm{A}$ & $\mathrm{N} / \mathrm{A}$ & 10.6 \\
\hline $\begin{array}{l}\text { GA-1-12\% BP } \\
2 / 249.5 \text { ton }\end{array}$ & 1795 & 3.504 & 2.852 & 461.6 & 393.3 & 312.827 & 393.7 & 0.983 & 0.797 & 2.97 & 4.86 & 93.52 & 4.71 & 90.62 & 14.80 & 46.28 & 18.61 & 10.6 \\
\hline & 1796 & 3.503 & 2.841 & 475.4 & 407.6 & 324.083 & 407.6 & 1.004 & 0.808 & 3.00 & 4.88 & 93.85 & 4.85 & 93.36 & 14.26 & 47.06 & 18.90 & 10.6 \\
\hline & 1797 & 3.503 & 2.836 & 452.1 & 387.6 & 308.178 & 388.4 & 0.962 & 0.776 & 2.98 & 4.83 & 92.92 & 4.82 & 92.76 & 14.27 & 47.12 & 19.04 & 10.6 \\
\hline & 1798 & 3.503 & 2.843 & 504.6 & 432.6 & 343.446 & 432.8 & 1.065 & 0.862 & 3.00 & 4.84 & 93.10 & 4.82 & 92.75 & 14.27 & 46.68 & 18.84 & 10.6 \\
\hline & 1799 & 3.503 & 2.847 & 502.9 & 431.3 & 342.064 & 431.3 & 1.057 & 0.861 & 3.01 & 4.83 & 92.95 & 4.80 & 92.32 & 14.24 & 46.19 & 18.73 & 10.6 \\
\hline & 1800 & 3.503 & 2.847 & 436.8 & 374.5 & 297.336 & 374.8 & 0.923 & 0.745 & 3.00 & 4.83 & 92.97 & 4.82 & 92.64 & 14.26 & 46.68 & 18.73 & 10.6 \\
\hline GA-1-14\%, CP & 1507 & 3.253 & N/A & 466.5 & N/A & N/A & $\mathrm{N} / \mathrm{A}$ & 1.123 & $\mathrm{~N} / \mathrm{A}$ & 3.05 & N/A & N/A & $\mathrm{N} / \mathrm{A}$ & N/A & N/A & N/A & N/A & 12.4 \\
\hline $\begin{array}{l}\text { GA-1-14\% BP } \\
2 / 249.5 \text { ton }\end{array}$ & 1801 & 3.504 & 2.846 & 464.7 & 396.0 & 315.082 & 396.1 & 0.967 & 0.782 & 3.04 & 4.89 & 94.00 & 4.86 & 93.39 & 14.78 & 46.64 & 18.78 & 12.4 \\
\hline & 1802 & 3.503 & 2.847 & 488.8 & 414.5 & 329.930 & 415.2 & 1.010 & 0.838 & 3.06 & 4.86 & 93.48 & 4.74 & 91.15 & 15.20 & 45.19 & 18.73 & 12.4 \\
\hline & 1803 & 3.503 & 2.847 & 488.4 & 414.0 & 329.431 & 414.9 & 1.006 & 0.820 & 3.07 & 4.84 & 93.15 & 4.84 & 93.04 & 15.23 & 46.15 & 18.73 & 12.4 \\
\hline & 1804 & 3.503 & 2.854 & 489.6 & 415.2 & 329.724 & 415.6 & 1.018 & 0.837 & 3.04 & 4.83 & 92.98 & 4.73 & 90.97 & 15.20 & 45.41 & 18.53 & 12.4 \\
\hline & 1805 & 3.503 & 2.860 & 487.0 & 412.6 & 327.884 & 413.6 & 1.007 & 0.825 & 3.06 & 4.81 & 92.57 & 4.75 & 91.33 & 15.28 & 45.38 & 18.36 & 12.4 \\
\hline & 1806 & 3.503 & 2.850 & 457.3 & 387.4 & 307.915 & 388.9 & 0.946 & 0.780 & 3.06 & 4.78 & 91.99 & 4.75 & 91.34 & 15.29 & 45.41 & 18.64 & 12.4 \\
\hline GA-2-0\%, CP & 1519 & 3.255 & 2.601 & 497.8 & 473.5 & 377.297 & 473.5 & 1.400 & 1.113 & 2.61 & 4.92 & 94.65 & 4.88 & 93.93 & 4.88 & 49.23 & 20.09 & 0.5 \\
\hline GA-2-0\%, CP & 1776 & 3.256 & 2.547 & 475.7 & 448.9 & 359.522 & 449.6 & 1.375 & 1.077 & 2.54 & 4.98 & 95.84 & 4.99 & 95.97 & 5.63 & 52.06 & 21.78 & 0.4 \\
\hline \begin{tabular}{|l}
$\mathrm{GA}-2-0 \%$ BP \\
$4 / 20,13.5$ ton
\end{tabular} & 2266 & 3.507 & 2.767 & 475.4 & 449.6 & 360.321 & 449.9 & 1.153 & 0.916 & 2.60 & 5.02 & 96.52 & 4.98 & 95.76 & 5.43 & 50.54 & 21.10 & 0.4 \\
\hline 18.9 ton & 2267 & 3.507 & 2.785 & 470.2 & 444.7 & 356.448 & 445.0 & 1.117 & 0.899 & 2.66 & 5.02 & 96.58 & 4.95 & 95.26 & 5.42 & 49.23 & 20.59 & 0.4 \\
\hline GA-2-4\%, CP & 1506 & 3.257 & 2.592 & 450.9 & 421.0 & 335.436 & 421.2 & 1.268 & 0.997 & 2.60 & 4.91 & 94.40 & 4.88 & 93.88 & 6.63 & 50.19 & 20.42 & 3.4 \\
\hline $\begin{array}{l}\mathrm{GA}-2-4 \% \mathrm{BP} \\
2 / 249.5 \text { ton } \\
\end{array}$ & 1807 & 3.505 & 2.792 & 422.2 & 379.9 & 301.170 & 380.6 & 0.980 & 0.814 & 2.72 & 4.78 & 91.98 & 4.65 & 89.43 & 10.02 & 47.29 & 20.34 & 3.4 \\
\hline & 1808 & 3.505 & 2.765 & 436.4 & 401.7 & 321.095 & 402.4 & 1.055 & 0.830 & 2.62 & 4.94 & 95.01 & 4.92 & 94.56 & 7.95 & 51.03 & 21.11 & 3.4 \\
\hline & 1809 & 3.505 & 2.782 & 472.4 & 435.5 & 347.649 & 435.8 & 1.130 & 0.894 & 2.64 & 4.94 & 95.01 & 4.89 & 94.02 & 7.81 & 50.15 & 20.63 & 3.4 \\
\hline & 1810 & 3.505 & 2.775 & 495.5 & 457.0 & 365.330 & 457.2 & 1.185 & 0.936 & 2.64 & 4.97 & 95.66 & 4.92 & 94.71 & 7.77 & 50.48 & 20.83 & 3.4 \\
\hline & 1811 & 3.505 & 2.758 & 425.9 & 392.8 & 313.577 & 392.9 & 1.035 & 0.815 & 2.60 & 4.95 & 95.23 & 4.92 & 94.64 & 7.77 & 51.23 & 21.31 & 3.4 \\
\hline & 1812 & 3.505 & 2.783 & 353.3 & 320.9 & 259.844 & 326.4 & 0.844 & 0.677 & 2.65 & 4.82 & 92.72 & 4.75 & 91.42 & 9.17 & 49.42 & 20.60 & 3.4 \\
\hline GA-2-6\%, CP & 1503 & 3.256 & 2.616 & 457.2 & 415.3 & 330.196 & 415.4 & 1.215 & 0.977 & 2.76 & 4.87 & 93.73 & 4.82 & 92.78 & 9.16 & 48.08 & 19.66 & 6.3 \\
\hline \begin{tabular}{|l|} 
GA-2-6\% BP \\
$2 / 249.5$ ton \\
\end{tabular} & 1813 & 3.505 & 2.804 & 403.9 & 365.6 & 291.100 & 365.8 & 0.930 & 0.747 & 2.75 & 4.89 & 94.12 & 4.84 & 92.98 & 9.48 & 48.58 & 20.00 & 6.3 \\
\hline & 1814 & 3.505 & 2.793 & 459.3 & 413.0 & 329.328 & 413.0 & 1.050 & 0.843 & 2.77 & 4.94 & 94.92 & 4.88 & 93.81 & 10.08 & 49.01 & 20.31 & 6.3 \\
\hline & 1815 & 3.505 & 2.793 & 481.0 & 432.0 & 344.929 & 432.1 & 1.105 & 0.864 & 2.75 & 4.96 & 95.30 & 4.98 & 95.74 & 10.19 & 50.34 & 20.31 & 6.3 \\
\hline & 1816 & 3.505 & 2.773 & 375.1 & 335.7 & 267.695 & 336.5 & 0.880 & 0.725 & 2.70 & 4.88 & 93.83 & 4.68 & 89.95 & 10.50 & 48.42 & 20.88 & 6.3 \\
\hline & 1817 & 3.505 & 2.800 & 479.3 & 430.4 & 342.942 & 430.5 & 1.093 & 0.881 & 2.77 & 4.92 & 94.53 & 4.84 & 93.08 & 10.20 & 48.55 & 20.11 & 6.3 \\
\hline & 1818 & 3.505 & 2.796 & 451.9 & 405.7 & 323.451 & 405.9 & 1.035 & 0.824 & 2.76 & 4.92 & 94.63 & 4.89 & 94.08 & 10.22 & 49.33 & 20.23 & 6.3 \\
\hline GA-2-8\%, CP & 1504 & 3.256 & 2.598 & 455.6 & 410.7 & 327.024 & 410.7 & 1.222 & 0.970 & 2.73 & 4.91 & 94.39 & 4.87 & 93.70 & 9.86 & 49.45 & 20.21 & 7.3 \\
\hline
\end{tabular}

Page 19 of 49 
Appendix F - Puck Data for Granulation Matrix Testing

\begin{tabular}{|c|c|c|c|c|c|c|c|c|c|c|c|c|c|c|c|c|c|c|}
\hline Batch ID & $\begin{array}{c}\text { Puck } \\
\text { ID }\end{array}$ & $\begin{array}{c}\text { Green } \\
\text { Puck } \\
\text { Diam. } \\
\text { (in.) } \\
\end{array}$ & $\begin{array}{c}\text { Sintered } \\
\text { Puck } \\
\text { Diameter } \\
\text { (in.) } \\
\end{array}$ & $\begin{array}{c}\text { Green } \\
\text { Weight } \\
\text { (g) }\end{array}$ & 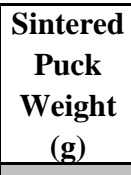 & $\begin{array}{c}\text { Puck } \\
\text { Suspended } \\
\text { Weight (g) }\end{array}$ & $\begin{array}{c}\text { Wet } \\
\text { Weight } \\
\text { (g) }\end{array}$ & $\begin{array}{c}\text { Green } \\
\text { Puck } \\
\text { Thick. } \\
\text { (in.) } \\
\end{array}$ & \begin{tabular}{|c|} 
Sintered \\
Puck \\
Thick. \\
(in.) \\
\end{tabular} & $\begin{array}{c}\text { Green } \\
\text { Density } \\
\text { (g/cc) }\end{array}$ & \begin{tabular}{|c} 
Archi- \\
medes \\
Density \\
$(\mathrm{g} / \mathrm{cc})$ \\
\end{tabular} & $\begin{array}{c}\text { Archi- } \\
\text { medes } \\
\text { Density } \\
(\% \mathrm{TD}) \\
\end{array}$ & $\begin{array}{c}\text { Geom. } \\
\text { Density } \\
\text { (g/cc) }\end{array}$ & $\begin{array}{l}\text { Geom. } \\
\text { (\%TD) }\end{array}$ & $\begin{array}{c}\text { Mass \% } \\
\text { Shrink- } \\
\text { age }\end{array}$ & $\begin{array}{c}\text { Volume } \\
\% \\
\text { Shinkage }\end{array}$ & \begin{tabular}{|c|} 
Diam. \\
$\%$ \\
Shrink- \\
age \\
\end{tabular} & $\begin{array}{l}\text { Powder } \\
\text { \% Mois }\end{array}$ \\
\hline $\begin{array}{l}\text { GA-2-8\% BP } \\
2 / 2414 \text { ton }\end{array}$ & 1819 & 3.505 & 2.830 & 452.3 & 403.0 & 321.973 & 403.0 & 0.975 & 0.791 & 2.93 & 4.97 & 95.65 & 4.94 & 95.02 & 10.90 & 47.10 & 19.26 & 7.3 \\
\hline & 1820 & 3.505 & 2.833 & 472.7 & 419.7 & 335.600 & 419.9 & 1.034 & 0.828 & 2.89 & 4.98 & 95.74 & 4.91 & 94.34 & 11.21 & 47.68 & 19.17 & 7.3 \\
\hline & \begin{tabular}{|l|}
1821 \\
\end{tabular} & 3.505 & 2.824 & 507.3 & 450.4 & 360.304 & 450.5 & 1.195 & 0.887 & 2.68 & 4.99 & 96.03 & 4.95 & 95.11 & 11.22 & 51.81 & 19.43 & 7.3 \\
\hline & 1822 & 3.505 & 2.826 & 455.1 & 404.0 & 323.084 & 404.0 & 0.984 & 0.784 & 2.92 & 4.99 & 96.02 & 5.01 & 96.38 & 11.23 & 48.20 & 19.37 & 7.3 \\
\hline & 1823 & 3.505 & 2.834 & 436.6 & 387.7 & 309.410 & 387.7 & 0.942 & 0.762 & 2.93 & 4.95 & 95.23 & 4.92 & 94.63 & 11.20 & 47.11 & 19.14 & 7.3 \\
\hline & 1824 & 3.505 & 2.824 & 399.6 & 355.0 & 283.151 & 355.0 & 0.865 & 0.705 & 2.92 & 4.94 & 95.02 & 4.90 & 94.32 & 11.16 & 47.08 & 19.43 & 7.3 \\
\hline GA-2- $12 \%$, CP & 1498 & 3.256 & 2.612 & 454.8 & 394.6 & 313.696 & 394.7 & 1.165 & 0.926 & 2.86 & 4.87 & 93.68 & 4.85 & 93.30 & 13.24 & 48.84 & 19.78 & 11.6 \\
\hline GA-2-12\% BP & 1850 & 3.502 & 2.805 & 364.9 & 311.4 & 248.224 & 311.5 & 0.784 & 0.639 & 2.95 & 4.92 & 94.64 & 4.81 & 92.52 & 14.66 & 47.70 & 19.90 & 11.6 \\
\hline & \begin{tabular}{|l|}
1851 \\
\end{tabular} & 3.501 & 2.812 & 407.3 & 347.7 & 276.756 & 347.7 & 0.875 & 0.698 & 2.95 & 4.90 & 94.25 & 4.89 & 94.10 & 14.63 & 48.53 & 19.68 & 11.6 \\
\hline & 1852 & 3.502 & 2.814 & 470.2 & 401.4 & 319.461 & 401.5 & 1.005 & 0.804 & 2.96 & 4.89 & 94.09 & 4.90 & 94.18 & 14.63 & 48.34 & 19.65 & 11.6 \\
\hline & 1853 & 3.502 & 2.808 & 394.2 & 336.4 & 268.017 & 336.7 & 0.843 & 0.690 & 2.96 & 4.90 & 94.19 & 4.80 & 92.36 & 14.66 & 47.37 & 19.82 & 11.6 \\
\hline & 1854 & 3.502 & 2.806 & 451.7 & 385.3 & 307.276 & 385.6 & 0.966 & 0.780 & 2.96 & 4.92 & 94.60 & 4.87 & 93.71 & 14.70 & 48.15 & 19.87 & 11.6 \\
\hline & 1855 & 3.502 & 2.804 & 388.2 & 331.2 & 263.643 & 331.4 & 0.843 & 0.664 & 2.92 & 4.89 & 94.00 & 4.93 & 94.76 & 14.68 & 49.49 & 19.93 & 11.6 \\
\hline GA-3-6\%, CP & 1515 & 3.254 & 2.606 & 498.7 & 461.3 & 366.210 & 462.1 & 1.364 & 1.099 & 2.68 & 4.81 & 92.51 & 4.80 & 92.32 & 7.50 & 48.31 & 19.91 & 4.8 \\
\hline \begin{tabular}{|l|} 
GA-3-6\% BP \\
$3 / 1010$ ton
\end{tabular} & 1857 & 3.504 & 2.803 & 443.5 & 403.8 & 321.215 & 403.9 & 1.030 & 0.828 & 2.72 & 4.88 & 93.92 & 4.82 & 92.72 & 8.95 & 48.55 & 20.01 & 4.8 \\
\hline & 1858 & 3.504 & 2.809 & 466.6 & 429.0 & 340.975 & 429.3 & 1.094 & 0.878 & 2.70 & 4.86 & 93.41 & 4.81 & 92.50 & 8.06 & 48.41 & 19.83 & 4.8 \\
\hline & 1859 & 3.505 & 2.804 & 469.5 & 431.9 & 343.700 & 432.1 & 1.097 & 0.885 & 2.71 & 4.89 & 93.96 & 4.82 & 92.72 & 8.01 & 48.36 & 20.00 & 4.8 \\
\hline & $\begin{array}{l}1860 \\
\end{array}$ & 3.504 & 2.799 & 486.3 & 447.4 & 355.995 & 447.6 & 1.136 & 0.906 & 2.71 & 4.88 & 93.92 & 4.90 & 94.15 & 8.00 & 49.10 & 20.12 & 4.8 \\
\hline $10-13$ ton & \begin{tabular}{|l|}
1861 \\
\end{tabular} & 3.505 & 2.837 & 456.1 & 419.6 & 334.336 & 420.2 & 1.019 & 0.839 & 2.83 & 4.89 & 93.98 & 4.83 & 92.82 & 8.00 & 46.05 & 19.06 & 4.8 \\
\hline & 1862 & 3.504 & 2.776 & 317.6 & 292.4 & 232.640 & 293.0 & 0.763 & 0.599 & 2.63 & 4.84 & 93.16 & 4.92 & 94.62 & 7.93 & 50.72 & 20.78 & 4.8 \\
\hline \begin{tabular}{|l|} 
GA-3-10\% \\
\end{tabular} & \begin{tabular}{|l|}
1510 \\
\end{tabular} & 3.255 & 2.617 & 519.5 & 462.7 & 367.212 & 462.7 & 1.362 & 1.093 & 2.80 & 4.85 & 93.19 & 4.80 & 92.33 & 10.93 & 48.12 & 19.60 & 7.5 \\
\hline GA-3-12\%, CP & 1514 & 3.254 & 2.645 & 458.8 & 400.0 & 317.624 & 400.5 & 1.153 & 0.930 & 2.92 & 4.83 & 92.82 & 4.78 & 91.83 & 12.82 & 46.70 & 18.72 & 10.0 \\
\hline $\begin{array}{l}\text { GA-3- } 12 \% \text { BP } \\
3 / 1010 \text { ton } \\
\end{array}$ & 1866 & 3.507 & 2.883 & 453.0 & 392.8 & 312.775 & 393.0 & 0.935 & 0.776 & 3.06 & 4.90 & 94.16 & 4.73 & 90.97 & 13.29 & 43.90 & 17.79 & 10.0 \\
\hline & \begin{tabular}{|l|}
1868 \\
\end{tabular} & 3.505 & 2.849 & 410.7 & 356.6 & 283.757 & 356.7 & 0.885 & 0.707 & 2.93 & 4.89 & 94.01 & 4.83 & 92.82 & 13.17 & 47.21 & 18.72 & 10.0 \\
\hline GA-3-14\%, CP & \begin{tabular}{|l|l|}
1516 \\
\end{tabular} & 3.253 & 2.620 & 499.4 & 429.6 & 341.865 & 430.0 & 1.245 & 0.996 & 2.94 & 4.87 & 93.74 & 4.88 & 93.86 & 13.98 & 48.10 & 19.46 & 10.5 \\
\hline $\begin{array}{l}\text { GA-3-14\% BP } \\
3 / 15 \text { 10 ton }\end{array}$ & 1869 & 3.505 & 2.811 & 494.6 & 439.0 & 349.093 & 439.1 & 1.105 & 0.900 & 2.83 & 4.88 & 93.80 & 4.79 & 92.21 & 11.24 & 47.60 & 19.80 & 7.5 \\
\hline & 1870 & 3.505 & 2.817 & 495.3 & 438.6 & 348.676 & 438.8 & 1.128 & 0.898 & 2.78 & 4.87 & 93.59 & 4.78 & 91.94 & 11.45 & 48.57 & 19.63 & 7.5 \\
\hline & \begin{tabular}{|l|}
1871 \\
\end{tabular} & 3.505 & 2.821 & 492.8 & 436.6 & 346.626 & 436.8 & 1.102 & 0.892 & 2.83 & 4.84 & 93.11 & 4.78 & 91.87 & 11.40 & 47.56 & 19.51 & 7.5 \\
\hline & 1872 & 3.505 & 2.819 & 489.0 & 433.7 & 344.085 & 434.1 & 1.108 & 0.881 & 2.79 & 4.82 & 92.66 & 4.81 & 92.53 & 11.31 & 48.56 & 19.57 & 7.5 \\
\hline & 1873 & 3.504 & 2.809 & 491.5 & 435.6 & 346.336 & 435.8 & 1.108 & 0.890 & 2.81 & 4.87 & 93.63 & 4.82 & 92.65 & 11.37 & 48.37 & 19.83 & 7.5 \\
\hline & 1874 & 3.505 & 2.788 & 460.1 & 415.3 & 33 & 415.4 & 1.071 & 0.854 & 2.72 & 4.88 & 93.82 & 4.86 & 93.45 & 9.74 & 49.54 & 20.46 & 7.5 \\
\hline GA-4-6\%, CP & 1518 & 3.254 & 2.585 & 495.1 & 454.9 & 361.410 & 455.2 & 1.366 & 1.090 & 2.66 & 4.85 & 93.27 & 4.85 & 93.29 & 8.12 & 49.63 & 20.56 & 4.9 \\
\hline GA-4-6\% BP & 1875 & 3.505 & 2.794 & 436.3 & 399.8 & 317.881 & 399.8 & 1.040 & 0.828 & 2.65 & 4.88 & 93.85 & 4.80 & 92.39 & 8.37 & 49.40 & 20.29 & 4.9 \\
\hline & \begin{tabular}{|l|}
1876 \\
\end{tabular} & 3.505 & 2.787 & 448.2 & 410.6 & 326.827 & 410.7 & 1.073 & 0.853 & 2.64 & 4.90 & 94.14 & 4.81 & 92.57 & 8.39 & 49.73 & 20.49 & 4.9 \\
\hline & \begin{tabular}{|l|l|}
1877 \\
\end{tabular} & 3.505 & 2.812 & 325.0 & 297.4 & 237.205 & 297.4 & 0.731 & 0.620 & 2.81 & 4.94 & 95.01 & 4.71 & 90.61 & 8.49 & 45.40 & 19.77 & 4.9 \\
\hline & 1878 & 3.505 & 2.792 & 459.5 & 420.3 & 334.286 & 420.3 & 1.089 & 0.875 & 2.67 & 4.89 & 93.97 & 4.79 & 92.04 & 8.53 & 49.01 & 20.34 & 4.9 \\
\hline & 1879 & 3.505 & 2.796 & 462.0 & 423.0 & 335.791 & 423.0 & 1.1 & 0.881 & 2. & 4. & 93.28 & 4.77 & 91.74 & 8.44 & 49.02 & 20.23 & 4.9 \\
\hline & \begin{tabular}{|l|}
1880 \\
\end{tabular} & 3.505 & 2.786 & 477.8 & 436.9 & 347.498 & 437.0 & 1.147 & 0.909 & 2.63 & 4.88 & 93.87 & 4.81 & 92.50 & 8.56 & 49.92 & 20.51 & 4.9 \\
\hline GA-4-8\%, CP & 1511 & 3.255 & N/A & 498.7 & 453.6 & N/A & N/A & 1.355 & N/A & 2.70 & $\mathrm{~N} / \mathrm{A}$ & N/A & N/A & N/A & 9.04 & N/A & N/A & 5.3 \\
\hline
\end{tabular}

Page 20 of 49 
Appendix F - Puck Data for Granulation Matrix Testing

\begin{tabular}{|c|c|c|c|c|c|c|c|c|c|c|c|c|c|c|c|c|c|c|}
\hline Batch ID & $\begin{array}{c}\text { Puck } \\
\text { ID }\end{array}$ & $\begin{array}{c}\text { Green } \\
\text { Puck } \\
\text { Diam. } \\
\text { (in.) }\end{array}$ & $\begin{array}{c}\text { Sintered } \\
\text { Puck } \\
\text { Diameter } \\
\text { (in.) }\end{array}$ & $\begin{array}{c}\text { Green } \\
\text { Weight } \\
\text { (g) }\end{array}$ & \begin{tabular}{|c|} 
Sintered \\
Puck \\
Weight \\
(g)
\end{tabular} & $\begin{array}{c}\text { Puck } \\
\text { Suspended } \\
\text { Weight (g) }\end{array}$ & $\begin{array}{c}\text { Wet } \\
\text { Weight } \\
\text { (g) }\end{array}$ & $\begin{array}{c}\text { Green } \\
\text { Puck } \\
\text { Thick. } \\
\text { (in.) }\end{array}$ & \begin{tabular}{|c|} 
Sintered \\
Puck \\
Thick. \\
(in.)
\end{tabular} & $\begin{array}{c}\text { Green } \\
\text { Density } \\
\text { (g/cc) }\end{array}$ & $\begin{array}{c}\text { Archi- } \\
\text { medes } \\
\text { Density } \\
\text { (g/cc) }\end{array}$ & $\begin{array}{c}\text { Archi- } \\
\text { medes } \\
\text { Density } \\
\text { (\%TD) }\end{array}$ & $\begin{array}{c}\text { Geom. } \\
\text { Density } \\
\text { (g/cc) }\end{array}$ & $\begin{array}{l}\text { Geom. } \\
\text { (\%TD) }\end{array}$ & $\begin{array}{c}\text { Mass \% } \\
\text { Shrink- } \\
\text { age }\end{array}$ & $\begin{array}{c}\text { Volume } \\
\% \\
\text { Shinkage }\end{array}$ & \begin{tabular}{|c|} 
Diam. \\
$\%$ \\
Shrink- \\
age
\end{tabular} & $\begin{array}{l}\text { Powder } \\
\% \text { Moist }\end{array}$ \\
\hline $\begin{array}{l}\text { GA-4-8\% BP } \\
3 / 1510 \text { ton }\end{array}$ & 1881 & 3.506 & 2.774 & 485.6 & 442.8 & 352.992 & 443.0 & 1.166 & 0.918 & 2.63 & 4.92 & 94.61 & 4.87 & 93.63 & 8.81 & 50.70 & 20.88 & 7.5 \\
\hline & 1882 & 3.505 & 2.775 & 490.1 & 446.8 & 355.964 & 447.0 & 1.166 & 0.920 & 2.66 & 4.91 & 94.38 & 4.90 & 94.20 & 8.83 & 50.53 & 20.83 & 7.5 \\
\hline & 1883 & 3.506 & 2.773 & 491.7 & 447.7 & 357.260 & 448.3 & 1.174 & 0.931 & 2.65 & 4.92 & 94.57 & 4.86 & 93.41 & 8.95 & 50.38 & 20.91 & 7.5 \\
\hline & 1884 & 3.506 & 2.780 & 488.5 & 445.7 & 355.206 & 446.3 & 1.164 & 0.924 & 2.65 & 4.89 & 94.09 & 4.85 & 93.23 & 8.76 & 50.08 & 20.71 & 7.5 \\
\hline GA-4-10\%, CP & 1512 & 3.254 & 2.600 & 487.1 & 433.5 & 343.394 & 433.8 & 1.291 & 1.039 & 2.77 & 4.80 & 92.21 & 4.79 & 92.19 & 11.00 & 48.61 & 20.10 & 7.4 \\
\hline GA-4-10\%, CP & 1533 & 3.258 & 2.568 & 498.8 & 448.3 & 356.543 & 448.7 & 1.387 & 1.100 & 2.63 & 4.86 & 93.55 & 4.80 & 92.31 & 10.12 & 50.72 & 21.18 & 6.2 \\
\hline $\begin{array}{l}\text { GA-4-10\% BP } \\
3 / 1510 \text { ton }\end{array}$ & 1885 & 3.505 & 2.792 & 472.5 & 425.1 & 338.856 & 425.3 & 1.100 & 0.881 & 2.72 & 4.92 & 94.57 & 4.81 & 92.46 & 10.03 & 49.17 & 20.34 & 6.9 \\
\hline & 1886 & 3.505 & 2.808 & 444.5 & 393.3 & 312.043 & 393.6 & 1.010 & 0.809 & 2.78 & 4.82 & 92.74 & 4.79 & 92.10 & 11.52 & 48.58 & 19.89 & 6.9 \\
\hline & 1887 & 3.504 & 2.811 & 466.3 & 411.5 & 326.482 & 411.6 & 1.055 & 0.848 & 2.80 & 4.83 & 92.97 & 4.77 & 91.73 & 11.75 & 48.26 & 19.78 & 6.9 \\
\hline & 1888 & 3.505 & 2.792 & 475.6 & 420.7 & 334.860 & 420.7 & 1.083 & 0.864 & 2.78 & 4.90 & 94.25 & 4.85 & 93.30 & 11.54 & 49.37 & 20.34 & 6.9 \\
\hline & 1889 & 3.506 & 2.806 & 481.1 & 426.1 & 338.349 & 426.3 & 1.095 & 0.878 & 2.78 & 4.84 & 93.17 & 4.79 & 92.07 & 11.43 & 48.63 & 19.97 & 6.9 \\
\hline & 1890 & 3.506 & 2.794 & 482.6 & 427.5 & 339.982 & 427.5 & 1.101 & 0.881 & 2.77 & 4.88 & 93.94 & 4.83 & 92.85 & 11.42 & 49.17 & 20.31 & 6.9 \\
\hline & 1891 & 3.505 & 2.767 & 487.3 & 434.6 & 346.002 & 434.6 & 1.143 & 0.915 & 2.70 & 4.91 & 94.33 & 4.82 & 92.67 & 10.81 & 50.10 & 21.06 & 6.9 \\
\hline & 1892 & 3.506 & 2.766 & 491.2 & 439.7 & 349.147 & 439.9 & 1.174 & 0.924 & 2.64 & 4.85 & 93.17 & 4.83 & 92.91 & 10.48 & 51.00 & 21.11 & 6.9 \\
\hline & 1893 & 3.506 & 2.750 & 487.9 & 437.6 & 348.395 & 437.7 & 1.179 & 0.932 & 2.62 & 4.90 & 94.23 & 4.82 & 92.74 & 10.31 & 51.36 & 21.56 & 6.9 \\
\hline & 1894 & 3.506 & 2.766 & 486.0 & 436.1 & 345.883 & 436.7 & 1.170 & 0.929 & 2.63 & 4.80 & 92.35 & 4.77 & 91.65 & 10.27 & 50.57 & 21.11 & 6.9 \\
\hline & 1895 & 3.506 & 2.755 & 485.5 & 435.4 & 346.620 & 435.7 & 1.169 & 0.917 & 2.62 & 4.89 & 94.00 & 4.86 & 93.44 & 10.32 & 51.55 & 21.42 & 6.9 \\
\hline & 1897 & 3.505 & 2.776 & 483.8 & 434.5 & 344.838 & 435.7 & 1.175 & 0.933 & 2.60 & 4.78 & 91.96 & 4.69 & 90.27 & 10.19 & 50.18 & 20.80 & 6.9 \\
\hline & 1898 & 3.506 & 2.774 & 485.9 & 436.3 & 346.215 & 437.0 & 1.180 & 0.938 & 2.60 & 4.81 & 92.42 & 4.70 & 90.29 & 10.21 & 50.23 & 20.88 & 6.9 \\
\hline & 1900 & 3.506 & 2.798 & 490.2 & 439.3 & 350.042 & 439.5 & 1.131 & 0.905 & 2.74 & 4.91 & 94.44 & 4.82 & 92.62 & 10.38 & 49.03 & 20.19 & 6.9 \\
\hline GA-4-12\%, CP & 1517 & 3.254 & N/A & 500.5 & 444.0 & N/A & N/A & 1.310 & N/A & 2.80 & $\mathrm{~N} / \mathrm{A}$ & N/A & $\mathrm{N} / \mathrm{A}$ & N/A & 11.29 & N/A & N/A & 7.1 \\
\hline $\begin{array}{l}\text { GA-4- } 12 \% \text { BP } \\
3 / 1510 \text { ton } \\
\end{array}$ & 1903 & 3.507 & 2.782 & 441.7 & 394.9 & 314.778 & 395.4 & 1.042 & 0.833 & 2.68 & 4.90 & 94.20 & 4.76 & 91.50 & 10.60 & 49.68 & 20.67 & 7.1 \\
\hline & 1904 & 3.506 & 2.804 & 438.3 & 389.5 & 310.184 & 391.7 & 1.020 & 0.832 & 2.72 & 4.78 & 91.89 & 4.62 & 88.94 & 11.13 & 47.82 & 20.02 & 7.1 \\
\hline & 1905 & 3.506 & 2.801 & 472.8 & 420.2 & 334.770 & 421.2 & 1.083 & 0.874 & 2.76 & 4.86 & 93.49 & 4.76 & 91.54 & 11.13 & 48.48 & 20.11 & 7.1 \\
\hline & 1906 & 3.506 & 2.784 & 502.2 & 445.8 & 355.579 & 446.2 & 1.158 & 0.920 & 2.74 & 4.92 & 94.60 & 4.86 & 93.39 & 11.23 & 49.90 & 20.59 & 7.1 \\
\hline & 1907 & 3.506 & 2.790 & 505.4 & 448.6 & 357.662 & 448.9 & 1.159 & 0.932 & 2.76 & 4.92 & 94.55 & 4.80 & 92.36 & 11.24 & 49.07 & 20.42 & 7.1 \\
\hline & 1908 & 3.507 & 2.802 & 518.8 & 461.3 & 367.720 & 462.2 & 1.192 & 0.948 & 2.75 & 4.88 & 93.89 & 4.81 & 92.58 & 11.08 & 49.22 & 20.10 & 7.1 \\
\hline $\begin{array}{l}\text { GA-4-12\%, CP } \\
\text { G3021-1 }\end{array}$ & 2089 & 3.254 & 2.546 & 498.5 & 447.5 & 356.694 & 448.0 & 1.392 & 1.102 & 2.63 & 4.90 & 94.25 & 4.87 & 93.58 & 10.23 & 51.53 & 21.76 & 5.4 \\
\hline GA-4-14\%, CP & 1513 & 3.255 & 2.612 & 499.4 & 436.8 & 347.775 & 437.5 & 1.278 & 1.034 & 2.87 & 4.87 & 93.62 & 4.81 & 92.49 & 12.54 & 47.89 & 19.75 & 9.3 \\
\hline $\begin{array}{l}\text { GA-4-14\% BP } \\
3 / 1510 \text { ton }\end{array}$ & 1909 & 3.504 & 2.805 & 494.3 & 433.6 & 345.374 & 434.4 & 1.112 & 0.890 & 2.81 & 4.87 & 93.66 & 4.81 & 92.49 & 12.28 & 48.70 & 19.95 & 9.3 \\
\hline & 1910 & 3.504 & 2.797 & 474.2 & 412.6 & 328.745 & 413.2 & 1.069 & 0.863 & 2.81 & 4.89 & 93.95 & 4.75 & 91.29 & 12.99 & 48.55 & 20.18 & 9.3 \\
\hline & 1911 & 3.504 & 2.799 & 479.4 & 416.8 & 332.120 & 418.7 & 1.065 & 0.856 & 2.85 & 4.81 & 92.58 & 4.83 & 92.84 & 13.06 & 48.70 & 20.12 & 9.3 \\
\hline & 1912 & 3.505 & 2.811 & 481.2 & 418.6 & 333.697 & 419.8 & 1.067 & 0.871 & 2.85 & 4.86 & 93.49 & 4.72 & 90.85 & 13.01 & 47.49 & 19.80 & 9.3 \\
\hline & 1913 & 3.504 & 2.812 & 501.4 & 437.2 & 348.541 & 438.9 & 1.117 & 0.898 & 2.84 & 4.84 & 93.05 & 4.78 & 91.97 & 12.80 & 48.21 & 19.75 & 9.3 \\
\hline GA-5-6\% & 1532 & 3.254 & 2.532 & 499.5 & 458.7 & 364.157 & 458.9 & 1.476 & 1.154 & 2.48 & 4.84 & 93.11 & 4.82 & 92.61 & 8.17 & 52.65 & 22.19 & 4.4 \\
\hline GA-5-10\% & 1536 & 3.253 & 2.559 & 495.2 & 447.3 & 354.342 & 447.8 & 1.421 & 1.129 & 2.56 & 4.79 & 92.04 & 4.70 & 90.37 & 9.67 & 50.82 & 21.33 & 5.5 \\
\hline
\end{tabular}

Page 21 of 49 
Appendix F - Puck Data for Granulation Matrix Testing

\begin{tabular}{|c|c|c|c|c|c|c|c|c|c|c|c|c|c|c|c|c|c|c|}
\hline Batch ID & $\begin{array}{c}\text { Puck } \\
\text { ID }\end{array}$ & $\begin{array}{c}\text { Green } \\
\text { Puck } \\
\text { Diam. } \\
\text { (in.) } \\
\end{array}$ & $\begin{array}{c}\text { Sintered } \\
\text { Puck } \\
\text { Diameter } \\
\text { (in.) } \\
\end{array}$ & $\begin{array}{c}\text { Green } \\
\text { Weight } \\
\text { (g) }\end{array}$ & \begin{tabular}{|c} 
Sintered \\
Puck \\
Weight \\
$(\mathrm{g})$ \\
\end{tabular} & $\begin{array}{c}\text { Puck } \\
\text { Suspended } \\
\text { Weight (g) }\end{array}$ & $\begin{array}{c}\text { Wet } \\
\text { Weight } \\
\text { (g) }\end{array}$ & $\begin{array}{c}\text { Green } \\
\text { Puck } \\
\text { Thick. } \\
\text { (in.) } \\
\end{array}$ & \begin{tabular}{|c|} 
Sintered \\
Puck \\
Thick. \\
(in.) \\
\end{tabular} & $\begin{array}{c}\text { Green } \\
\text { Density } \\
\text { (g/cc) }\end{array}$ & \begin{tabular}{|c} 
Archi- \\
medes \\
Density \\
$(\mathrm{g} / \mathrm{cc})$ \\
\end{tabular} & $\begin{array}{c}\text { Archi- } \\
\text { medes } \\
\text { Density } \\
(\% \mathrm{TD}) \\
\end{array}$ & $\begin{array}{c}\text { Geom. } \\
\text { Density } \\
\text { (g/cc) }\end{array}$ & $\begin{array}{l}\text { Geom. } \\
\text { (\%TD) }\end{array}$ & $\begin{array}{c}\text { Mass \% } \\
\text { Shrink- } \\
\text { age }\end{array}$ & $\begin{array}{c}\text { Volume } \\
\% \\
\text { Shinkage }\end{array}$ & \begin{tabular}{|c|} 
Diam. \\
$\%$ \\
Shrink- \\
age \\
\end{tabular} & $\begin{array}{l}\text { Powder } \\
\% \text { Mois }\end{array}$ \\
\hline $\begin{array}{l}\text { GA-5-12\%, } \\
\text { G0518-0 Full, } \\
\text { BP 7/10.4.9 tons }\end{array}$ & 2804 & 3.508 & 2.730 & 493.9 & 422.6 & 336.916 & 422.7 & 1.145 & 0.900 & 2.72 & 4.93 & 94.74 & 4.89 & 94.11 & 14.44 & 52.39 & 22.18 & 9.4 \\
\hline 5.8 tons & 2805 & 3.510 & 2.704 & 496.2 & 421.6 & 336.312 & 421.7 & 1.151 & 0.903 & 2.72 & 4.94 & 94.95 & 4.96 & 95.38 & 15.03 & 53.43 & 22.96 & 9.4 \\
\hline 4.4 tons & 2806 & 3.507 & 2.643 & 459.7 & 383.3 & 304.906 & 383.4 & 1.144 & 0.868 & 2.54 & 4.88 & 93.91 & 4.91 & 94.43 & 16.62 & 56.90 & 24.64 & 9.4 \\
\hline 6.4 tons & 2807 & 3.508 & 2.688 & 478.6 & 398.5 & 317.089 & 398.6 & 1.149 & 0.882 & 2.63 & 4.89 & 94.02 & 4.86 & 93.41 & 16.74 & 54.92 & 23.38 & 9.4 \\
\hline 7.4 tons & 2808 & 3.507 & 2.700 & 494.6 & 409.4 & 326.473 & 409.5 & 1.154 & 0.892 & 2.71 & 4.93 & 94.83 & 4.89 & 94.04 & 17.23 & 54.18 & 23.01 & 9.4 \\
\hline 7 tons & 2809 & 3.509 & 2.691 & 486.9 & 406.2 & 323.576 & 406.3 & 1.158 & 0.892 & 2.65 & 4.91 & 94.43 & 4.88 & 93.93 & 16.57 & 54.69 & 23.31 & 9.4 \\
\hline GA-7/4-10\% & 1528 & 3.254 & 2.545 & 500.1 & 435.2 & 343.330 & 440.8 & 1.500 & 1.200 & 2.45 & 4.46 & 85.86 & 4.35 & 83.64 & 12.98 & 51.05 & 21.79 & 7.9 \\
\hline GA-8/4-10\% & 1527 & 3.258 & 2.502 & 468.6 & 405.3 & 320.000 & 406.7 & 1.438 & 1.096 & 2.39 & 4.67 & 89.90 & 4.59 & 88.24 & 13.51 & 55.04 & 23.20 & 7.5 \\
\hline GA-8/4-12\% & 1624 & 3.253 & 2.577 & 499.8 & 420.1 & 333.074 & 420.9 & 1.296 & 1.032 & 2.83 & 4.78 & 91.99 & 4.76 & 91.56 & 15.95 & 50.02 & 20.78 & 9.3 \\
\hline GA-9-8\% & 1508 & 3.259 & 2.538 & 439.3 & 392.8 & 311.565 & 395.1 & 1.311 & 1.025 & 2.45 & 4.70 & 90.43 & 4.62 & 88.87 & 10.59 & 52.57 & 22.12 & 6.5 \\
\hline GA-11-6\% & 1502 & 3.255 & 2.598 & 502.1 & 437.4 & 349.658 & 437.7 & 1.300 & 1.034 & 2.83 & 4.97 & 95.54 & 4.87 & 93.62 & 12.89 & 49.32 & 20.18 & 6.2 \\
\hline \begin{tabular}{|l|} 
GA-11-8\% \\
\end{tabular} & 1501 & 3.255 & 2.600 & 512.6 & 437.7 & 349.324 & 437.8 & 1.291 & 1.042 & 2.91 & 4.95 & 95.14 & 4.83 & 92.82 & 14.61 & 48.49 & 20.12 & 8.1 \\
\hline GA-11-10\%, CP & 1531 & 3.254 & 2.620 & 500.1 & 419.7 & 334.073 & 420.5 & 1.227 & 0.989 & 2.99 & 4.86 & 93.39 & 4.80 & 92.34 & 16.08 & 47.74 & 19.48 & 9.7 \\
\hline $\begin{array}{l}\text { GA-11-10\% BP } \\
2 / 24.9 .5 \text { ton }\end{array}$ & 1789 & 3.503 & 2.815 & 409.9 & 343.6 & 273.904 & 344.9 & 0.860 & 0.708 & 3.02 & 4.84 & 93.07 & 4.76 & 91.48 & 16.17 & 46.83 & 19.64 & 9.7 \\
\hline & 1790 & 3.503 & 2.805 & 479.0 & 401.1 & 319.241 & 401.9 & 1.013 & 0.815 & 2.99 & 4.85 & 93.32 & 4.86 & 93.43 & 16.26 & 48.40 & 19.93 & 9.7 \\
\hline 8.5 ton & 1791 & 3.501 & 2.812 & 476.6 & 398.8 & 317.675 & 399.9 & 1.009 & 0.824 & 2.99 & 4.85 & 93.27 & 4.75 & 91.43 & 16.32 & 47.31 & 19.68 & 9.7 \\
\hline 9.5 ton & 1792 & 3.502 & 2.816 & 457.8 & 383.1 & 305.216 & 384.4 & 0.973 & 0.793 & 2.98 & 4.84 & 93.04 & 4.73 & 91.00 & 16.32 & 47.29 & 19.59 & 9.7 \\
\hline & 1793 & 3.502 & 2.807 & 451.1 & 377.7 & 301.222 & 377.9 & 0.953 & 0.770 & 3.00 & 4.93 & 94.73 & 4.84 & 92.99 & 16.27 & 48.08 & 19.85 & 9.7 \\
\hline & 1794 & 3.503 & 2.800 & 286.3 & 240.1 & 191.338 & 240.1 & 0.613 & 0.489 & 2.96 & 4.92 & 94.69 & 4.86 & 93.55 & 16.14 & 49.02 & 20.07 & 9.7 \\
\hline \begin{tabular}{|l|} 
GA-13-6\% \\
\end{tabular} & 1538 & 3.255 & 2.576 & 555.4 & 504.3 & 401.934 & 504.6 & 1.514 & 1.205 & 2.69 & 4.91 & 94.46 & 4.90 & 94.21 & 9.20 & 50.14 & 20.86 & 2.8 \\
\hline GA-13-10\% & 1539 & 3.254 & N/A & 527.8 & N/A & N/A & N/A & 1.329 & N/A & 2.91 & N/A & N/A & N/A & N/A & N/A & N/A & N/A & 8.8 \\
\hline \begin{tabular}{|l|} 
GA- $13-12 \%$, CP \\
\end{tabular} & 1623 & 3.252 & 2.621 & 499.4 & 418.6 & 333.725 & 420.1 & 1.223 & 1.000 & 3.00 & 4.85 & 93.20 & 4.73 & 91.02 & 16.18 & 46.88 & 19.40 & 10.8 \\
\hline $\begin{array}{l}\text { GA- } 13-12 \% \text { BP } \\
3 / 1610 \text { ton }\end{array}$ & 1916 & 3.507 & 2.829 & 456.5 & 382.8 & 305.380 & 384.0 & 0.961 & 0.778 & 3.00 & 4.87 & 93.63 & 4.78 & 91.83 & 16.14 & 47.31 & 19.33 & 10.9 \\
\hline & 1917 & 3.504 & 2.826 & 466.0 & 389.6 & 310.952 & 391.0 & 0.979 & 0.784 & 3.01 & 4.87 & 93.60 & 4.83 & 92.95 & 16.39 & 47.90 & 19.35 & 10.9 \\
\hline & 1918 & 3.496 & 2.819 & 460.4 & 384.9 & 306.698 & 386.0 & 0.972 & 0.785 & 3.01 & 4.85 & 93.34 & 4.79 & 92.16 & 16.40 & 47.48 & 19.36 & 10.9 \\
\hline & 1919 & 3.498 & 2.818 & 461.7 & 385.7 & 307.714 & 387.0 & 0.974 & 0.778 & & 4.86 & 93.55 & & 93.25 & 16.46 & & 19.44 & 10.9 \\
\hline & 1920 & 3.504 & 2.817 & 475.1 & 397.2 & 316.332 & 398.0 & 1.005 & 0.805 & 2.99 & 4.86 & 93.53 & 4.83 & 92.88 & 16.40 & 48.22 & 19.61 & 10.9 \\
\hline & 1921 & 3.503 & 2.836 & & 343.8 & 274.069 & 345.9 & 0.880 & 0.715 & 2.96 & 4.79 & 92.04 & 4.64 & 89.30 & 16.47 & 46.74 & 19.04 & 10.9 \\
\hline GA-13-14\% & 1540 & 3.254 & 2.624 & 498.3 & 416.8 & 331.498 & 418.1 & 1.207 & 0.990 & 3.03 & 4.81 & 92.55 & 4.75 & 91.33 & 16.36 & 46.65 & 19.36 & 11.3 \\
\hline $\begin{array}{l}\text { GA-14-10\% Full } \\
\text { Batch }\end{array}$ & 1530 & 3.254 & 2.585 & 499.3 & 448.6 & 355.194 & 448.6 & 1.387 & 1.100 & 2.64 & 4.80 & 92.36 & 4.74 & 91.16 & 10.15 & 49.94 & 20.56 & 6.7 \\
\hline GA-14-14\% & 1535 & 3.253 & 2.582 & 499.2 & 431.7 & 342.687 & 431.9 & 1.309 & 1.045 & 2.80 & 4.84 & 93.06 & 4.81 & 92.56 & 13.52 & 49.70 & 20.63 & 9.2 \\
\hline GA-15-10\%, CP & 1534 & 3.253 & 2.581 & 499.3 & 442.7 & 350.536 & 442.7 & 1.366 & 1.090 & 2.68 & 4.80 & 92.37 & 4.74 & 91.07 & 11.34 & 49.76 & 20.66 & 5.8 \\
\hline $\begin{array}{l}\mathrm{GA}-15-10 \% \mathrm{BP} \\
3 / 1610 \text { ton }\end{array}$ & 1922 & 3.507 & 2.821 & 524.1 & 464.0 & 369.226 & 464.3 & 1.171 & 0.945 & 2.83 & 4.88 & 93.85 & 4.79 & 92.16 & 11.47 & 47.77 & 19.56 & 5.8 \\
\hline & 1923 & 3.508 & 2.778 & 498.8 & 442.8 & 351.649 & 443.1 & 1.162 & 0.926 & 2.71 & 4.84 & 93.11 & 4.81 & 92.56 & 11.23 & 50.02 & 20.81 & 5.8 \\
\hline & 1924 & 3.507 & 2.795 & 501.3 & 445.1 & & 446.1 & 1.189 & 0.941 & 2.66 & 4.78 & 91.99 & 4. & 90.44 & 11.21 & 49.72 & 20.30 & 50 \\
\hline & 1925 & 3.506 & 2.777 & 499.9 & 443.9 & 352.863 & 444.7 & 1.164 & 0.927 & 2.71 & 4.83 & 92.95 & 4.82 & 92.75 & 11.20 & 50.03 & 20.79 & 5.8 \\
\hline & 1926 & 3.507 & 2.778 & 499.9 & 443.9 & 352.611 & 444.8 & 1.169 & 0.927 & 2.70 & 4.82 & 92.60 & 4.82 & 92.69 & 11.20 & 50.23 & 20.79 & 5.8 \\
\hline
\end{tabular}

Page 22 of 49 
Appendix F - Puck Data for Granulation Matrix Testing

\begin{tabular}{|c|c|c|c|c|c|c|c|c|c|c|c|c|c|c|c|c|c|c|}
\hline Batch ID & $\begin{array}{c}\text { Puck } \\
\text { ID }\end{array}$ & $\begin{array}{c}\text { Green } \\
\text { Puck } \\
\text { Diam. } \\
\text { (in.) } \\
\end{array}$ & $\begin{array}{l}\text { Sintered } \\
\text { Puck } \\
\text { Diameter } \\
\text { (in.) } \\
\end{array}$ & $\begin{array}{c}\text { Green } \\
\text { Weight } \\
\text { (g) }\end{array}$ & $\begin{array}{c}\text { Sintered } \\
\text { Puck } \\
\text { Weight } \\
(\mathrm{g}) \\
\end{array}$ & $\begin{array}{c}\text { Puck } \\
\text { Suspended } \\
\text { Weight (g) }\end{array}$ & $\begin{array}{c}\text { Wet } \\
\text { Weight } \\
\text { (g) }\end{array}$ & $\begin{array}{c}\text { Green } \\
\text { Puck } \\
\text { Thick. } \\
\text { (in.) } \\
\end{array}$ & $\begin{array}{c}\text { Sintered } \\
\text { Puck } \\
\text { Thick. } \\
\text { (in.) } \\
\end{array}$ & $\begin{array}{c}\text { Green } \\
\text { Density } \\
(\mathrm{g} / \mathrm{cc})\end{array}$ & \begin{tabular}{|c|} 
Archi- \\
medes \\
Density \\
(g/cc) \\
\end{tabular} & $\begin{array}{c}\text { Archi- } \\
\text { medes } \\
\text { Density } \\
(\% \text { TD) }\end{array}$ & $\begin{array}{c}\text { Geom. } \\
\text { Density } \\
(\mathrm{g} / \mathrm{cc})\end{array}$ & $\begin{array}{l}\text { Geom. } \\
\text { (\%TD) }\end{array}$ & $\begin{array}{c}\text { Mass \% } \\
\text { Shrink- } \\
\text { age }\end{array}$ & $\begin{array}{c}\text { Volume } \\
\% \\
\text { Shinkage }\end{array}$ & \begin{tabular}{|c|} 
Diam. \\
$\%$ \\
Shrink- \\
age \\
\end{tabular} & $\begin{array}{l}\text { Powder } \\
\text { \% Moist }\end{array}$ \\
\hline GA-15-14\%, CP & 1529 & 3.255 & 2.600 & 498.5 & 427.1 & 339.221 & 427.5 & 1.283 & 1.032 & 2.85 & \begin{tabular}{|l|}
4.84 \\
\end{tabular} & 93.04 & 4.76 & 91.45 & 14.32 & 48.67 & 20.12 & 9.5 \\
\hline $\begin{array}{l}\text { GA-15-14\% BP } \\
3 / 1610 \text { ton } \\
\end{array}$ & 1927 & 3.505 & 2.804 & 484.7 & 421.1 & 334.412 & 422.3 & 1.100 & 0.875 & 2.79 & 4.79 & 92.14 & 4.75 & 91.43 & 13.12 & 49.08 & 20.00 & 8.3 \\
\hline & 1928 & 3.509 & N/A & 477.7 & N/A & N/A & N/A & 1.044 & N/A & 2.89 & $\mathrm{~N} / \mathrm{A}$ & $\mathrm{N} / \mathrm{A}$ & N/A & $\mathrm{N} / \mathrm{A}$ & $\mathrm{N} / \mathrm{A}$ & N/A & N/A & 8.3 \\
\hline & 1929 & 3.510 & 2.798 & 503.7 & 428.1 & 340.400 & 429.3 & 1.100 & 0.927 & 2.89 & 4.82 & 92.61 & 4.58 & 88.11 & 15.01 & 46.44 & 20.28 & 8.3 \\
\hline & 1930 & 3.508 & 2.864 & 509.8 & 437.0 & 347.731 & 438.6 & 1.043 & 0.890 & 3.09 & 4.81 & 92.48 & 4.65 & 89.42 & 14.28 & 43.11 & 18.36 & 8.3 \\
\hline & 1931 & 3.508 & 2.849 & 516.9 & 443.5 & 353.406 & 444.6 & 1.068 & 0.873 & 3.06 & 4.86 & 93.52 & 4.86 & 93.49 & 14.20 & 46.08 & 18.79 & 8.3 \\
\hline & 1932 & 3.511 & N/A & 458.5 & N/A & N/A & N/A & 1.080 & N/A & 2.68 & N/A & N/A & N/A & N/A & N/A & N/A & N/A & 8.3 \\
\hline \begin{tabular}{|l} 
GA-15-15\% \\
\end{tabular} & 1537 & 3.254 & 2.594 & 499.6 & 420.5 & 333.222 & 421.0 & 1.277 & 1.019 & 2.87 & 4.79 & 92.12 & 4.76 & 91.61 & 15.83 & 49.28 & 20.28 & 10.2 \\
\hline GA-16-8\% & 1618 & 3.252 & 2.520 & 454.6 & 413.2 & 328.988 & 413.7 & 1.354 & 1.052 & 2.47 & 4.88 & 93.80 & 4.80 & 92.39 & 9.11 & 53.34 & 22.51 & 3.9 \\
\hline \begin{tabular}{|l} 
GA-16-14\% \\
\end{tabular} & 1615 & 3.255 & 2.521 & 498.0 & 437.3 & 349.067 & 437.5 & 1.408 & 1.092 & 2.59 & 4.94 & 95.10 & 4.89 & 94.12 & 12.19 & 53.47 & 22.55 & 5.8 \\
\hline GA-17-10\% & 1544 & 3.254 & 2.572 & 497.8 & 449.1 & 355.411 & 451.6 & 1.475 & 1.158 & 2.48 & 4.67 & 89.79 & 4.55 & 87.57 & 9.78 & 50.94 & 20.96 & 3.7 \\
\hline GA-18-10\% & 1619 & 3.254 & 2.590 & 612.8 & 547.1 & 434.089 & 547.8 & 1.700 & 1.351 & 2.64 & 4.81 & 92.53 & 4.69 & 90.17 & 10.72 & 49.64 & 20.41 & 6.9 \\
\hline \begin{tabular}{|l|} 
GA-18-12\% \\
\end{tabular} & 1620 & 3.254 & 2.577 & 500.7 & 440.5 & 349.500 & 441.3 & 1.376 & 1.095 & 2.67 & 4.80 & 92.28 & 4.71 & 90.48 & 12.02 & 50.08 & 20.81 & 8.1 \\
\hline \begin{tabular}{|l} 
GA-18-14\% \\
\end{tabular} & 1616 & 3.254 & 2.570 & 498.8 & 430.4 & 342.345 & 430.6 & 1.328 & 1.060 & 2.76 & 4.88 & 93.78 & 4.78 & 91.83 & 13.71 & 50.20 & 21.02 & 9.1 \\
\hline GA-19-4\% & 1543 & 3.254 & 2.589 & 498.9 & 460.8 & 366.733 & 460.9 & 1.392 & 1.099 & 2.63 & 4.89 & 94.10 & 4.86 & 93.44 & 7.64 & 50.01 & 20.44 & 3.8 \\
\hline \begin{tabular}{|l} 
GA-19-8\% \\
\end{tabular} & 1621 & 3.254 & 2.590 & 501.0 & 447.5 & 357.186 & 447.8 & 1.335 & 1.053 & 2.75 & 4.94 & 94.97 & 4.92 & 94.63 & 10.68 & 50.02 & 20.41 & 7.4 \\
\hline GA-19-10\%, CP & 1625 & 3.255 & N/A & 497.2 & N/A & N/A & N/A & 1.267 & N/A & 2.88 & N/A & N/A & N/A & N/A & N/A & N/A & N/A & 8.9 \\
\hline $\begin{array}{l}\text { GA- } 19-10 \% \text { BP } \\
3 / 21 \quad 10 \text { ton } \\
\end{array}$ & 1945 & 3.505 & 2.813 & 477.9 & 420.1 & 334.539 & 420.6 & 1.063 & 0.858 & 2.84 & 4.88 & 93.87 & 4.81 & 92.43 & 12.09 & 48.00 & 19.74 & 8.9 \\
\hline & 1946 & 3.504 & 2.815 & 480.3 & 420.9 & 335.032 & 421.3 & 1.056 & 0.852 & 2.88 & 4.88 & 93.83 & 4.84 & 93.12 & 12.37 & 47.92 & 19.66 & 8.9 \\
\hline & 1947 & 3.505 & 2.817 & 481.0 & 421.4 & 335.105 & 421.4 & 1.055 & 0.853 & 2.88 & 4.88 & 93.91 & 4.84 & 92.99 & 12.39 & 47.76 & 19.63 & 8.9 \\
\hline $\begin{array}{l}\text { GA-19-10\% } \\
\text { (Repeat) }\end{array}$ & 1662 & 3.255 & 2.609 & 497.8 & 438.1 & 348.870 & 438.1 & 1.288 & 1.026 & 2.83 & 4.91 & 94.42 & 4.87 & 93.70 & 11.99 & 48.81 & 19.85 & 9.4 \\
\hline GA-19-12\%, CP & 1541 & 3.254 & 2.595 & 499.7 & 429.5 & 341.595 & 429.9 & 1.284 & 1.026 & 2.86 & 4.86 & 93.54 & 4.83 & 92.86 & 14.05 & 49.17 & 20.25 & 10.7 \\
\hline $\begin{array}{l}\text { GA-19-12\% BP } \\
3 / 2110 \text { ton } \\
\end{array}$ & 1950 & 3.505 & 2.785 & 477.4 & 412.5 & 328.602 & 412.8 & 1.063 & 0.855 & 2.84 & 4.90 & 94.21 & 4.83 & 92.91 & 13.59 & 49.21 & 20.54 & 10.7 \\
\hline & 1951 & 3.504 & 2.782 & 483.8 & 415.2 & 330.598 & 415.4 & 1.086 & 0.865 & 2.82 & 4.90 & 94.16 & 4.82 & 92.64 & 14.18 & 49.78 & 20.61 & 10.7 \\
\hline & 1952 & 3.505 & 2.783 & 489.5 & 419.4 & 334.008 & 419.7 & 1.088 & 0.872 & 2.85 & 4.89 & 94.12 & 4.82 & 92.76 & 14.32 & 49.46 & 20.60 & 10.7 \\
\hline & 1955 & 3.503 & 2.787 & 486.1 & 414.1 & 330.017 & 414.4 & 1.073 & 0.860 & 2.87 & 4.91 & 94.37 & 4.82 & 92.60 & 14.81 & 49.26 & 20.44 & 10.7 \\
\hline GA-19-14\%, CP & 1622 & 3.253 & 2.596 & 501.1 & 422.6 & 337.329 & 423.1 & 1.259 & 1.009 & 2.92 & 4.93 & 94.75 & 4.83 & 92.83 & 15.67 & 48.95 & 20.20 & 11.8 \\
\hline \begin{tabular}{|l|}
$\mathrm{GA}-19-14 \% \mathrm{BP}$ \\
$3 / 2110$ ton
\end{tabular} & 1956 & 3.502 & 2.804 & 461.7 & 388.6 & 310.956 & 389.3 & 0.977 & 0.807 & 2.99 & 4.96 & 95.39 & 4.76 & 91.48 & 15.83 & 47.04 & 19.93 & 11.8 \\
\hline & 1957 & 3.501 & 2.810 & 479.0 & 403.1 & 322.142 & 404.0 & 1.003 & 0.815 & 3.03 & 4.92 & 94.70 & 4.87 & 93.57 & 15.85 & 47.64 & 19.74 & 11.8 \\
\hline & 1958 & 3.501 & 2.805 & 480.9 & 404.6 & & 405.6 & 1.014 & 0.825 & & 4.91 & 94.50 & 4. & 93.11 & 15.87 & 47.76 & 19.88 & 11.8 \\
\hline & 1959 & 3.502 & 2.790 & 488.5 & 411.1 & 327.899 & 412.0 & 1.058 & 0.854 & 2.92 & 4.89 & 94.00 & 4.80 & 92.37 & 15.84 & 48.76 & 20.33 & 11.8 \\
\hline & 1960 & 3.501 & 2.808 & 486.7 & 409.8 & 327.192 & 410.7 & 1.023 & 0.832 & 3.02 & 4.91 & 94.37 & 4.85 & 93.31 & 15.80 & 47.67 & 19.79 & 11.8 \\
\hline \begin{tabular}{|l|} 
GA-20 \\
\end{tabular} & 1642 & 3.256 & 2.556 & 449.0 & 418.6 & 336.651 & 418.6 & 1.320 & 1.028 & 2.49 & 5.11 & 98.23 & 4.84 & 93.10 & 6.77 & 52.00 & 21.50 & 0.5 \\
\hline $\begin{array}{l}\text { GA-20-0\% BP } \\
4 / 20.9 .3 \text { ton }\end{array}$ & 2256 & 3.505 & 2.736 & 439.6 & 407.2 & 326.180 & 407.3 & 1.070 & 0.851 & 2.60 & 5.02 & 96.53 & 4.97 & 95.48 & 7.37 & 51.53 & 21.94 & 0.4 \\
\hline 9.8 ton & 2257 & 3.505 & 2.734 & 461.0 & 427.4 & 342.543 & 427.8 & 1.142 & 0.902 & 2.55 & 5.01 & 96.41 & 4.92 & 94.69 & 7.29 & 51.93 & 22.00 & 0.4 \\
\hline 7 ton & 2258 & 3.504 & 2.719 & 452.5 & 420.8 & & 421.0 & 1.450 & 0.890 & 1.9 & 5.1 & 96.69 & 4.97 & 95.53 & 1.01 & 63.03 & 22.40 & 0.4 \\
\hline 6 ton & 2259 & 3.504 & 2.722 & 430.9 & 394.5 & 315.897 & 394.8 & 1.070 & 0.842 & 2.55 & 5.00 & 96.15 & 4.91 & 94.46 & 8.45 & 52.50 & 22.32 & 0.4 \\
\hline $\begin{array}{l}6 \text { ton, } 0.2 \text { pre- } \\
\text { press }\end{array}$ & 2260 & 3.506 & 2.710 & 380.5 & 350.4 & 280.378 & 350.5 & 0.971 & 0.755 & 2.48 & 5.00 & 96.10 & 4.91 & 94.40 & 7.91 & 53.54 & 22.70 & 0.4 \\
\hline
\end{tabular}

Page 23 of 49 
Appendix F - Puck Data for Granulation Matrix Testing

\begin{tabular}{|c|c|c|c|c|c|c|c|c|c|c|c|c|c|c|c|c|c|c|}
\hline Batch ID & $\begin{array}{l}\text { Puck } \\
\text { ID }\end{array}$ & $\begin{array}{c}\text { Green } \\
\text { Puck } \\
\text { Diam. } \\
\text { (in.) }\end{array}$ & $\begin{array}{c}\text { Sintered } \\
\text { Puck } \\
\text { Diameter } \\
\text { (in.) }\end{array}$ & $\begin{array}{c}\text { Green } \\
\text { Weight } \\
\text { (g) }\end{array}$ & $\begin{array}{c}\text { Sintered } \\
\text { Puck } \\
\text { Weight } \\
\text { (g) }\end{array}$ & $\begin{array}{c}\text { Puck } \\
\text { Suspended } \\
\text { Weight (g) }\end{array}$ & $\begin{array}{c}\text { Wet } \\
\text { Weight } \\
\text { (g) }\end{array}$ & $\begin{array}{c}\text { Green } \\
\text { Puck } \\
\text { Thick. } \\
\text { (in.) }\end{array}$ & $\begin{array}{c}\text { Sintered } \\
\text { Puck } \\
\text { Thick. } \\
\text { (in.) }\end{array}$ & $\begin{array}{c}\text { Green } \\
\text { Density } \\
\text { (g/cc) }\end{array}$ & \begin{tabular}{|c} 
Archi- \\
medes \\
Density \\
$(\mathrm{g} / \mathrm{cc})$
\end{tabular} & $\begin{array}{l}\text { Archi- } \\
\text { medes } \\
\text { Density } \\
(\% \mathrm{TD})\end{array}$ & $\begin{array}{c}\text { Geom. } \\
\text { Density } \\
\text { (g/cc) }\end{array}$ & $\begin{array}{l}\text { Geom. } \\
\text { (\% TD) }\end{array}$ & $\begin{array}{c}\text { Mass \% } \\
\text { Shrink- } \\
\text { age }\end{array}$ & $\begin{array}{c}\text { Volume } \\
\% \\
\text { Shinkage }\end{array}$ & \begin{tabular}{|c} 
Diam. \\
$\%$ \\
Shrink- \\
age
\end{tabular} & $\begin{array}{l}\text { Powder } \\
\text { \% Mois }\end{array}$ \\
\hline \begin{tabular}{|l|} 
GA-20-8\%, CP \\
\end{tabular} & \begin{tabular}{|l|}
1626 \\
\end{tabular} & 3.254 & 2.580 & 499.2 & 435.1 & 345.666 & 435.1 & 1.320 & 1.050 & 2.77 & 4.87 & 93.56 & 4.84 & 92.99 & 12.84 & 49.99 & 20.71 & 7.5 \\
\hline $\begin{array}{l}\text { GA- } 20-8 \% \text { BP } \\
3 / 2110 \text { ton }\end{array}$ & 1962 & 3.508 & 2.792 & 463.4 & 403.1 & 322.825 & 403.2 & 1.013 & 0.808 & 2.89 & 5.02 & 96.45 & 4.97 & 95.60 & 13.01 & 49.46 & 20.41 & 7.2 \\
\hline & 1964 & 3.506 & 2.795 & 479.4 & 416.3 & 333.395 & 416.5 & 1.049 & 0.836 & 2.89 & 5.01 & 96.33 & 4.95 & 95.22 & 13.16 & 49.34 & 20.28 & 7.2 \\
\hline & 1965 & 3.506 & 2.796 & 478.4 & 415.4 & 332.426 & 415.5 & 1.049 & 0.838 & 2.88 & 5.00 & 96.16 & 4.93 & 94.72 & 13.17 & 49.18 & 20.25 & 7.2 \\
\hline GA-20-10\%, CP & 1771 & 3.255 & 2.569 & 500.4 & 423.9 & 336.765 & 424.1 & 1.317 & 1.034 & 2.79 & 4.85 & 93.34 & 4.82 & 92.79 & 15.29 & 51.09 & 21.08 & 9.8 \\
\hline $\begin{array}{l}\text { GA-20-10\% BP } \\
3 / 2110 \text { ton }\end{array}$ & 1967 & 3.506 & 2.784 & 437.7 & 373.2 & 298.529 & 373.4 & 0.961 & 0.770 & 2.88 & 4.98 & 95.86 & 4.86 & 93.41 & 14.74 & 49.47 & 20.59 & 8.6 \\
\hline & \begin{tabular}{|l|}
1969 \\
\end{tabular} & 3.506 & 2.786 & 38.8 & 372.5 & 297.611 & 372.5 & 0.953 & 0.760 & 2.91 & 4.97 & 95.65 & 4.90 & 94.32 & 15.11 & 49.63 & 20.54 & 8.6 \\
\hline & 1970 & 3.507 & 2.784 & 438.8 & 372.5 & 297.373 & 372.5 & 0.950 & 0.765 & 2.92 & 4.96 & 95.35 & 4.88 & 93.84 & 15.11 & 49.24 & 20.62 & 8.6 \\
\hline & \begin{tabular}{|l|}
1971 \\
\end{tabular} & 3.504 & 2.744 & 428.7 & 363.8 & 288.715 & 363.8 & 0.996 & 0.783 & 2.72 & 4.85 & 93.18 & 4.79 & 92.17 & 15.14 & 51.78 & 21.69 & 8.6 \\
\hline & 1972 & 3.506 & 2.740 & 470.5 & 401.1 & 319.035 & 401.2 & 1.085 & 0.860 & 2.74 & 4.88 & 93.88 & 4.83 & 92.80 & 14.75 & 51.58 & 21.85 & 8.6 \\
\hline GA-20-12\% & 1627 & 3.252 & 2.575 & 499.2 & 422.3 & 336.023 & 422.5 & 1.285 & 1.016 & 2.85 & 4.88 & 93.91 & 4.87 & 93.64 & 15.40 & 50.42 & 20.82 & 10.6 \\
\hline GA-22-10\%, CP & 1628 & 3.254 & 2.530 & 500.2 & 428.1 & 339.627 & 429.3 & 1.428 & 1.093 & 2.57 & 4.77 & 91.81 & 4.75 & 91.40 & 14.41 & 53.72 & 22.25 & 8.5 \\
\hline $\begin{array}{l}\text { GA-22-10\% BP } \\
3 / 2110 \text { ton }\end{array}$ & 1973 & 3.507 & 2.724 & 478.1 & 407.5 & 325.704 & 407.5 & 1.127 & 0.868 & 2.68 & 4.98 & 95.81 & 4.91 & 94.51 & 14.77 & 53.53 & 22.33 & 7.8 \\
\hline & 1974 & 3.506 & 2.725 & 490.4 & 417.5 & 333.564 & 417.9 & 1.150 & 0.888 & 2.70 & 4.95 & 95.20 & 4.92 & 94.58 & 14.87 & 53.34 & 22.28 & 7.8 \\
\hline & 1975 & 3.507 & 2.725 & 490.5 & 417.8 & 333.740 & 418.0 & 1.156 & 0.900 & 2.68 & 4.96 & 95.36 & 4.86 & 93.38 & 14.82 & 52.99 & 22.30 & 7.8 \\
\hline & 1976 & 3.506 & 2.728 & 480.9 & 409.8 & 327.012 & 409.8 & 1.128 & 0.877 & 2.69 & 4.95 & 95.19 & 4.88 & 93.79 & 14.78 & 52.92 & 22.19 & 7.8 \\
\hline GA-22-12\%, CP & 1629 & 3.253 & N/A & 499.0 & N/A & N/A & N/A & 1.355 & N/A & 2.70 & N/A & N/A & N/A & N/A & N/A & N/A & N/A & 10.4 \\
\hline $\begin{array}{l}\text { GA- } 22-12 \% \text { BP } \\
3 / 21 \quad 10 \text { ton }\end{array}$ & 1984 & 3.506 & 2.741 & 467.4 & 389.1 & 310.465 & 389.1 & 1.065 & 0.821 & 2.77 & 4.95 & 95.16 & 4.90 & 94.23 & 16.75 & 52.87 & 21.82 & 10.4 \\
\hline & 1985 & 3.506 & 2.731 & 469.7 & 391.3 & 312.655 & 391.7 & 1.060 & 0.828 & 2.80 & 4.95 & 95.20 & 4.92 & 94.65 & 16.69 & 52.60 & 22.10 & 10.4 \\
\hline on side & 1986 & 3.506 & 2.740 & 493.6 & 411.3 & 328.294 & 411.5 & 1.120 & 0.868 & 2.79 & 4.94 & 93.44 & 4.90 & 94.28 & 16.67 & 52.66 & 21.85 & 10.4 \\
\hline 7 ton & 1987 & 3.504 & 2.672 & 488.9 & 408.0 & 324.129 & 408.1 & 1.213 & 0.931 & 2.55 & 4.86 & 94.13 & 4.77 & 91.69 & 16.55 & 55.36 & 23.74 & 10.4 \\
\hline 8 ton & 1990 & 3.508 & 2.723 & 470.0 & 396.8 & 311.670 & 392.0 & 1.093 & 0.857 & 2.71 & 4.94 & 94.99 & 4.8 & 93.28 & 15.57 & 52.75 & 22.38 & 10.4 \\
\hline 10 ton & 1993 & 3.508 & 2.715 & 461.2 & 383.0 & & 383.7 & 1.064 & 0.815 & 2.74 & 4.93 & 94.77 & 4.95 & 95.23 & 16.96 & 54.11 & 22.61 & 10.4 \\
\hline 7 ton & 1995 & 3.507 & 2.648 & 458.4 & 380.9 & 302.151 & 380.9 & 1.175 & 0.879 & 2.46 & 4.84 & 93.02 & 4.80 & 92.31 & 16.91 & 57.34 & 24.49 & 10.4 \\
\hline \begin{tabular}{|l} 
on side, 7.5 ton \\
\end{tabular} & 1997 & 3.509 & 2.708 & 451.8 & 375.2 & 164 & 375.2 & 1.053 & 0.810 & 2.71 & 4.93 & 94.89 & 4.91 & 94.35 & 16.95 & 54.18 & 22.83 & 10.4 \\
\hline 7.25 ton & 2000 & 3.507 & 2.657 & 456.2 & 379.1 & 300.211 & 379.2 & 1.164 & 0.880 & 2.48 & 4.80 & 92.30 & 4.74 & 91.15 & 16.90 & 56.60 & 24.24 & 10.4 \\
\hline \begin{tabular}{|l} 
GA-22-1 \\
\end{tabular} & 1834 & 3.256 & 2.499 & & 418.6 & & 418.6 & 1.425 & 1.087 & 2.57 & 4.84 & 92.98 & & & 16.20 & 55.06 & 23.25 & 10.4 \\
\hline \begin{tabular}{|l} 
GA-22-14\%, CP \\
\end{tabular} & 1630 & 3.253 & 2.526 & 499.6 & 414.4 & 329.067 & 414.5 & 1.360 & 1.049 & 2.70 & 4.85 & 93.28 & 4.81 & 92.48 & 17.05 & 53.48 & 22.35 & 10.4 \\
\hline $\begin{array}{l}\text { GA-22-14\% BP } \\
3 / 21 \quad 10 \text { ton }\end{array}$ & 1978 & 3.506 & 2.707 & 463.8 & 382.4 & 304.288 & 382.4 & 1.081 & 0.840 & 2.71 & 4.90 & 94.14 & 4.83 & 92.80 & 17.55 & 53.67 & 22.79 & 11.9 \\
\hline & 1979 & 3.505 & 2.711 & 484.6 & 398.5 & 316.861 & 398.5 & 1.126 & 0.875 & 2.72 & 4.88 & 93.87 & 4.81 & 92.56 & 17.77 & 53.50 & 22.65 & 11.9 \\
\hline & \begin{tabular}{|l|}
1980 \\
\end{tabular} & 3.505 & 2.715 & 497.3 & 407.8 & & 407.8 & 1.155 & 0.906 & 2.72 & 4.86 & 93.37 & 4.74 & 91.21 & 18.00 & 52.93 & 22.54 & 11.9 \\
\hline & 1981 & 3.506 & 2.718 & 501.9 & 412.5 & 327.328 & 412.5 & 1.175 & 0.907 & 2.70 & 4.84 & 93.14 & 4.78 & 91.96 & 17.81 & 53.60 & 22.48 & 11.9 \\
\hline & 1982 & 3.504 & 2.760 & & 401.3 & & 401.5 & 1.081 & 0.860 & 2.8 & 4. & 94.13 & 4. & 91.50 & 17.80 & 50.63 & 21.23 & 11.9 \\
\hline & 1983 & 3.504 & 2.727 & 390.3 & 320.4 & 255.716 & 321.4 & 0.868 & 0.692 & 2.85 & 4.88 & 93.81 & 4.84 & 93.00 & 17.91 & 51.70 & 22.17 & 11.9 \\
\hline GA-23-6\%, CP & 1832 & 3.255 & 2.590 & 569.8 & 523.8 & 416.727 & 528.3 & 1.640 & 1.304 & 2.55 & 4.69 & 90.28 & 4.65 & 89.45 & 8.07 & 49.65 & 20.43 & 3.6 \\
\hline $\begin{array}{l}\text { GA-23-6\% BP } \\
3 / 2210 \text { ton } \\
\end{array}$ & 2004 & 3.504 & 2.746 & 481.5 & 440.7 & 350.672 & 441.3 & 1.190 & 0.938 & 2.56 & 4.86 & 93.51 & 4.84 & 93.07 & 8.47 & 51.58 & 21.63 & 4.0 \\
\hline & 2005 & 3.506 & 2.752 & 486.7 & 446.0 & 354.980 & 447.0 & 1.206 & 0.948 & 2.55 & 4.85 & 93.21 & 4.83 & 92.79 & 8.36 & 51.56 & 21.51 & 4.0 \\
\hline oloin & 2006 & 3.506 & 2.762 & 485.3 & 445.2 & 354.153 & 447.8 & 1.208 & 0.959 & 2.54 & 4.75 & 91.42 & 4.73 & 90.90 & 8.26 & 50.72 & 21.22 & 4.0 \\
\hline
\end{tabular}

Page 24 of 49 
Appendix F - Puck Data for Granulation Matrix Testing

\begin{tabular}{|c|c|c|c|c|c|c|c|c|c|c|c|c|c|c|c|c|c|c|}
\hline Batch ID & $\begin{array}{c}\text { Puck } \\
\text { ID }\end{array}$ & $\begin{array}{c}\text { Green } \\
\text { Puck } \\
\text { Diam. } \\
\text { (in.) }\end{array}$ & $\begin{array}{l}\text { Sintered } \\
\text { Puck } \\
\text { Diameter } \\
\text { (in.) }\end{array}$ & $\begin{array}{c}\text { Green } \\
\text { Weight } \\
\text { (g) }\end{array}$ & $\begin{array}{c}\text { Sintered } \\
\text { Puck } \\
\text { Weight } \\
\text { (g) }\end{array}$ & $\begin{array}{c}\text { Puck } \\
\text { Suspended } \\
\text { Weight (g) }\end{array}$ & $\begin{array}{c}\text { Wet } \\
\text { Weight } \\
\text { (g) }\end{array}$ & $\begin{array}{c}\text { Green } \\
\text { Puck } \\
\text { Thick. } \\
\text { (in.) }\end{array}$ & $\begin{array}{c}\text { Sintered } \\
\text { Puck } \\
\text { Thick. } \\
\text { (in.) }\end{array}$ & $\begin{array}{c}\text { Green } \\
\text { Density } \\
(\mathrm{g} / \mathrm{cc})\end{array}$ & $\begin{array}{c}\text { Archi- } \\
\text { medes } \\
\text { Density } \\
(\mathrm{g} / \mathrm{cc})\end{array}$ & $\begin{array}{l}\text { Archi- } \\
\text { medes } \\
\text { Density } \\
(\% \mathrm{TD})\end{array}$ & $\begin{array}{c}\text { Geom. } \\
\text { Density } \\
(\mathrm{g} / \mathrm{cc})\end{array}$ & $\begin{array}{l}\text { Geom. } \\
\text { (\%TD) }\end{array}$ & $\begin{array}{c}\text { Mass \% } \\
\text { Shrink- } \\
\text { age }\end{array}$ & $\begin{array}{c}\text { Volume } \\
\% \\
\text { Shinkage }\end{array}$ & \begin{tabular}{|c|} 
Diam. \\
$\%$ \\
Shrink- \\
age
\end{tabular} & $\begin{array}{l}\text { Powder } \\
\% \text { Moist }\end{array}$ \\
\hline GA-23-8\%, CP & 1631 & 3.253 & N/A & 501.0 & N/A & $\mathrm{N} / \mathrm{A}$ & N/A & 1.372 & N/A & 2.68 & N/A & N/A & N/A & N/A & N/A & N/A & N/A & 8.0 \\
\hline GA-23-8\% BP & 2009 & 3.506 & 2.806 & 495.3 & 444.6 & 353.887 & 445.1 & 1.137 & 0.906 & 2.75 & 4.87 & 93.74 & 4.84 & 93.10 & 10.24 & 48.95 & 19.97 & 5.7 \\
\hline 12 ton & 2010 & 3.506 & 2.785 & 496.0 & 445.2 & 354.026 & 445.4 & 1.156 & 0.915 & 2.71 & 4.87 & 93.70 & 4.87 & 93.70 & 10.24 & 50.05 & 20.56 & 5.7 \\
\hline 10 ton & 2011 & 3.506 & 2.820 & 497.9 & 447.3 & 355.812 & 448.8 & 1.143 & 0.916 & 2.75 & 4.81 & 92.51 & 4.77 & 91.72 & 10.16 & 48.14 & 19.57 & 5.7 \\
\hline \begin{tabular}{|l|} 
GA-23-10\%, CP \\
\end{tabular} & 1632 & 3.252 & N/A & 499.3 & N/A & N/A & N/A & 1.362 & N/A & 2.69 & N/A & N/A & N/A & N/A & N/A & N/A & N/A & 8.6 \\
\hline \begin{tabular}{|l|}
$\mathrm{GA}-23-10 \% \mathrm{BP}$ \\
$3 / 227$ ton
\end{tabular} & 2014 & 3.507 & 2.682 & 445.1 & 377.1 & N/A & N/A & 1.146 & 0.868 & 2.45 & N/A & N/A & 4.69 & 90.22 & 15.28 & 55.69 & 23.52 & 8.6 \\
\hline & $\frac{2015}{2016}$ & $\frac{3.507}{3.507}$ & $\frac{2.690}{2.671}$ & $\begin{array}{l}448.3 \\
467.0\end{array}$ & $\begin{array}{l}382.7 \\
3987\end{array}$ & $\frac{302.148}{315.244}$ & $\begin{array}{l}383.5 \\
398.7\end{array}$ & $\begin{array}{l}1.161 \\
1.213\end{array}$ & $\begin{array}{l}0.879 \\
0.919\end{array}$ & $\frac{2.44}{2.43}$ & $\frac{4.70}{4.78}$ & $\begin{array}{l}90.47 \\
91.87\end{array}$ & $\begin{array}{l}4.67 \\
4.72\end{array}$ & $\begin{array}{l}89.87 \\
90.84\end{array}$ & \begin{tabular}{|l|}
14.63 \\
14.63
\end{tabular} & $\begin{array}{l}55.45 \\
56.04\end{array}$ & $\frac{23.30}{23.84}$ & $\begin{array}{l}8.6 \\
86\end{array}$ \\
\hline & 2017 & 3.507 & 2.700 & 467.6 & 399.6 & N/A & N/A & 1.211 & 0.925 & 2.44 & N/A & N/A & 4.60 & 88.52 & 14.54 & 54.72 & 23.01 & 8.6 \\
\hline \begin{tabular}{|l|} 
GA-23-12\%, CP \\
Full (G0229-2)
\end{tabular} & 1835 & 3.255 & 2.539 & 499.6 & 421.5 & 334.280 & 425.6 & 1.423 & 1.106 & 2.57 & 4.62 & 88.76 & 4.59 & 88.31 & 15.63 & 52.70 & 22.00 & 9.2 \\
\hline $\begin{array}{l}\text { GA-23-12\% } \\
\text { (G0229-2) BP } \\
\end{array}$ & 2041 & 3.507 & 2.709 & 496.7 & 415.7 & 328.030 & 419.2 & 1.265 & 0.965 & 2.48 & 4.56 & 87.68 & 4.56 & 87.68 & 16.31 & 54.47 & 22.75 & 9.2 \\
\hline & 2042 & 3.507 & 2.715 & 497.5 & 416.5 & 328.417 & 421.0 & 1.261 & 0.970 & 2.49 & 4.50 & 86.51 & 4.52 & 87.01 & 16.28 & 53.89 & 22.58 & 9.2 \\
\hline & 2043 & 3.507 & 2.687 & 498.4 & 416.5 & 328.511 & 418.0 & 1.263 & 0.961 & 2.49 & 4.65 & 89.50 & 4.66 & 89.67 & 16.43 & 55.33 & 23.38 & 9.2 \\
\hline & 2045 & 3.506 & 2.698 & 497.8 & 416.2 & 329.348 & 419.9 & 1.276 & 0.972 & 2.47 & 4.60 & 88.39 & 4.57 & 87.87 & 16.39 & 54.88 & 23.05 & 9.2 \\
\hline 7.4 ton & 2049 & 3.507 & 2.686 & 496.3 & 413.6 & 326.487 & 414.8 & 1.261 & 0.958 & 2.49 & 4.68 & 90.06 & 4.65 & 89.39 & 16.66 & 55.43 & 23.41 & 9.2 \\
\hline \begin{tabular}{|l|}
$\mathrm{GA}-23-12 \% \mathrm{CP}$ \\
$(\mathrm{G} 0306-0)$
\end{tabular} & 1934 & 3.255 & 2.519 & 499.4 & 422.9 & 334.072 & 422.9 & 1.429 & 1.098 & 2.56 & 4.76 & 91.56 & 4.71 & 90.67 & 15.32 & 53.97 & 22.61 & 10.4 \\
\hline $\begin{array}{l}\text { GA-23-12\% CP } \\
\text { (G0306-1) }\end{array}$ & 1935 & 3.254 & N/A & 484.4 & N/A & N/A & N/A & 1.395 & N/A & 2.55 & N/A & N/A & N/A & N/A & N/A & N/A & N/A & \\
\hline $\begin{array}{l}\text { GA-23-12\% CP } \\
\text { (G0306-2) }\end{array}$ & 1936 & 3.255 & N/A & 499.9 & N/A & N/A & N/A & 1.398 & N/A & 2.62 & N/A & N/A & N/A & N/A & N/A & N/A & N/A & 4.4 \\
\hline $\begin{array}{l}\text { GA-23-12\% } \\
\text { (G0306-2 \& } \\
\text { G0126-1) BP } \\
\text { 3/22 7.3 ton }\end{array}$ & 2055 & 3.506 & 2.698 & 501.0 & 423.4 & 336.264 & 425.1 & 1.253 & 0.960 & 2.53 & 4.77 & 91.66 & 4.71 & 90.50 & 15.49 & 54.62 & 23.05 & 10.5 \\
\hline \begin{tabular}{|l|l}
7.4 ton \\
\end{tabular} & 2056 & 3.505 & 2.715 & 497.3 & 419.6 & 332.918 & 422.1 & 1.238 & 0.945 & 2.54 & 4.70 & 90.48 & 4.68 & 89.98 & 15.62 & 54.19 & 22.54 & 10.5 \\
\hline 7 ton & 2058 & 3.506 & 2.691 & & 407.6 & 322.082 & 4 & 1.223 & 0.927 & 2.51 & 4.72 & 90.81 & 4.72 & 90.70 & 15.96 & 55.34 & 23.25 & 10.5 \\
\hline 7.1 ton & 2059 & 3.506 & 2.674 & 477.0 & 399.6 & 316.729 & 399.6 & 1.202 & 0.907 & 2.51 & 4.82 & 92.73 & 4.79 & 92.04 & 16.23 & 56.11 & 23.73 & 10.5 \\
\hline 7.2 ton & 2060 & 3.505 & 2.680 & 500.5 & 419.6 & 332.159 & 419.7 & 1.261 & 0.947 & 2.51 & 4.79 & 92.18 & 4.79 & 92.15 & 16.16 & 56.09 & 23.54 & 10.5 \\
\hline 7 ton & 2064 & 3.505 & 2.707 & 495.3 & 416.0 & 329.140 & 419.0 & 1.255 & 0.965 & 2.50 & 4.63 & 89.03 & 4.57 & 87.87 & 16.01 & 54.13 & 22.77 & 10.5 \\
\hline & 2065 & 3.505 & 2.690 & 497.0 & 417.1 & $\mathrm{~N} / \mathrm{A}$ & N/A & 1.253 & 0.957 & 2.51 & N/A & N/A & 4.68 & 89.97 & 16.08 & 55.00 & 23.25 & 10.5 \\
\hline & 2066 & 3.505 & 2.679 & 494.8 & 414.4 & 327.567 & 414.4 & 1.245 & 0.939 & 2.51 & 4.77 & 91.78 & 4.78 & 91.85 & 16.25 & 55.93 & 23.57 & 10.5 \\
\hline & 2067 & 3.504 & 2.680 & 497.8 & 417.1 & 329.115 & 417.2 & 1.251 & 0.958 & 2.52 & 4.74 & 91.06 & 4.71 & 90.55 & 16.21 & 55.19 & 23.52 & 10.5 \\
\hline & 2068 & 3.503 & 2.680 & 495.5 & 415.0 & 328.334 & 415.0 & 1.244 & 0.940 & 2.52 & 4.79 & 92.09 & 4.77 & 91.82 & 16.25 & 55.76 & 23.49 & 10.5 \\
\hline & 2069 & 3.504 & N/A & 490.3 & N/A & N/A & N/A & \begin{tabular}{|l|}
1.240 \\
\end{tabular} & 0.933 & 2.50 & N/A & N/A & N/A & N/A & N/A & N/A & N/A & 10.5 \\
\hline \begin{tabular}{|l|} 
GA-23-12\%, CP \\
$($ G0316-0)
\end{tabular} & 1941 & 3.256 & 2.540 & 457.8 & 386.3 & 305.537 & 386.3 & 1.253 & 0.969 & 2.68 & 4.78 & 91.98 & 4.80 & 92.30 & 15.62 & 52.93 & 21.99 & \\
\hline $\begin{array}{l}\begin{array}{l}\text { GA-23-12\% } \\
\text { (G0316-1) }\end{array} \\
\end{array}$ & 1943 & 3.255 & 2.501 & 437.4 & 368.7 & 292.091 & 369.3 & 1.250 & 0.954 & 2.57 & 4.78 & 91.83 & 4.80 & 92.29 & 15.71 & 54.93 & 23.16 & 9.6 \\
\hline GA-24-8\% CP & 1826 & 3.256 & 2.535 & 463.3 & 407.9 & 321.561 & \begin{tabular}{|l|}
407.9 \\
\end{tabular} & \begin{tabular}{|l|}
1.360 \\
\end{tabular} & 1.052 & 2.50 & 4.72 & 90.85 & 4.69 & 90.13 & 11.96 & 53.10 & 22.14 & 6.1 \\
\hline
\end{tabular}

Page 25 of 49 
Appendix F - Puck Data for Granulation Matrix Testing

\begin{tabular}{|c|c|c|c|c|c|c|c|c|c|c|c|c|c|c|c|c|c|c|}
\hline Batch ID & $\begin{array}{c}\text { Puck } \\
\text { ID }\end{array}$ & $\begin{array}{c}\text { Green } \\
\text { Puck } \\
\text { Diam. } \\
\text { (in.) }\end{array}$ & $\begin{array}{c}\text { Sintered } \\
\text { Puck } \\
\text { Diameter } \\
\text { (in.) }\end{array}$ & $\begin{array}{c}\text { Green } \\
\text { Weight } \\
\text { (g) }\end{array}$ & \begin{tabular}{|c|} 
Sintered \\
Puck \\
Weight \\
$(\mathrm{g})$ \\
\end{tabular} & $\begin{array}{c}\text { Puck } \\
\text { Suspended } \\
\text { Weight (g) }\end{array}$ & $\begin{array}{c}\text { Wet } \\
\text { Weight } \\
\text { (g) }\end{array}$ & $\begin{array}{c}\text { Green } \\
\text { Puck } \\
\text { Thick. } \\
\text { (in.) }\end{array}$ & $\begin{array}{c}\text { Sintered } \\
\text { Puck } \\
\text { Thick. } \\
\text { (in.) }\end{array}$ & $\begin{array}{c}\text { Green } \\
\text { Density } \\
\text { (g/cc) }\end{array}$ & $\begin{array}{c}\text { Archi- } \\
\text { medes } \\
\text { Density } \\
\text { (g/cc) }\end{array}$ & $\begin{array}{c}\text { Archi- } \\
\text { medes } \\
\text { Density } \\
(\% \mathrm{TD})\end{array}$ & $\begin{array}{l}\text { Geom. } \\
\text { Density } \\
\text { (g/cc) }\end{array}$ & $\begin{array}{l}\text { Geom. } \\
\text { (\%TD) }\end{array}$ & $\begin{array}{c}\text { Mass \% } \\
\text { Shrink- } \\
\text { age }\end{array}$ & $\begin{array}{c}\text { Volume } \\
\% \\
\text { Shinkage }\end{array}$ & \begin{tabular}{|c|} 
Diam. \\
$\%$ \\
Shrink- \\
age
\end{tabular} & $\begin{array}{l}\text { Powder } \\
\% \text { Moist }\end{array}$ \\
\hline $\begin{array}{l}\text { GA-24-8\% BP } \\
3 / 227 \text { ton }\end{array}$ & 2020 & 3.508 & 2.703 & 441.8 & 386.9 & 305.117 & 388.1 & 1.157 & 0.892 & 2.41 & 4.66 & 89.66 & 4.61 & 88.68 & 12.43 & 54.22 & 22.95 & 5.8 \\
\hline & 2021 & 3.507 & 2.698 & 465.0 & 408.2 & 323.001 & 412.3 & 1.255 & 0.959 & 2.34 & 4.57 & 87.91 & 4.54 & 87.35 & 12.22 & 54.77 & 23.07 & 5.8 \\
\hline & 2022 & 3.507 & 2.702 & 463.9 & 407.1 & 322.659 & 412.4 & 1.259 & 0.958 & 2.33 & 4.54 & 87.24 & 4.52 & 86.94 & 12.24 & 54.82 & 22.95 & 5.8 \\
\hline GA-24-10\% CP & 1836 & 3.255 & 2.541 & 498.3 & 428.0 & & & 1.444 & 1.119 & 2.53 & N/A & N/A & 4.60 & 88.49 & 14.11 & 52.77 & 21.94 & 8.3 \\
\hline $\begin{array}{l}\text { GA-24-10\% BP } \\
3 / 227 \text { ton }\end{array}$ & 2025 & 3.505 & 2.677 & 472.1 & 399.1 & N/A & N/A & 1.220 & 0.933 & 2.45 & N/A & N/A & 4.64 & 89.16 & 15.46 & 55.38 & 23.62 & 8.3 \\
\hline & 2026 & 3.506 & 2.668 & 491.2 & 415.0 & 328.209 & 415.7 & 1.269 & 0.953 & 2.45 & 4.74 & 91.22 & 4.75 & 91.38 & 15.51 & 56.50 & 23.90 & 8.3 \\
\hline & 2027 & 3.506 & 2.683 & 488.2 & 413.3 & 327.634 & 415.9 & 1.260 & 0.963 & 2.45 & 4.68 & 90.05 & 4.63 & 89.06 & 15.34 & 55.23 & 23.47 & 8.3 \\
\hline GA-24-12\% CP & 1827 & 3.256 & 2.523 & 500.0 & 417.5 & 330.763 & 418.9 & 1.410 & 1.083 & 2.60 & 4.74 & 91.10 & 4.70 & 90.46 & 16.50 & 53.87 & 22.51 & 9.7 \\
\hline $\begin{array}{l}\text { GA-24-12\% BP } \\
3 / 22,7 \text { ton, } \\
\text { G0224-1 }\end{array}$ & 2031 & 3.506 & 2.673 & 476.3 & 395.7 & N/A & N/A & 1.207 & 0.912 & 2.49 & N/A & N/A & 4.72 & 90.71 & 16.92 & 56.07 & 23.76 & 9.5 \\
\hline & 2032 & 3.506 & 2.667 & 498.6 & 413.6 & 327.431 & 414.4 & 1.268 & 0.962 & 2.49 & 4.76 & 91.46 & 4.69 & 90.29 & 17.05 & 56.09 & 23.93 & 9.5 \\
\hline & 2033 & 3.506 & 2.699 & 497.1 & 412.0 & 327.432 & 417.0 & 1.257 & 0.975 & 2.50 & 4.60 & 88.46 & 4.51 & 86.65 & 17.12 & 54.02 & 23.02 & 9.5 \\
\hline GA-24-14\% CP & 1828 & 3.256 & 2.552 & 499.2 & 414.3 & 327.900 & 417.5 & 1.409 & 1.089 & 2.60 & 4.62 & 88.92 & 4.54 & 87.26 & 17.01 & 52.51 & 21.62 & 11.1 \\
\hline $\begin{array}{l}\text { GA-24-14\%, } \\
\text { G0224-2, BP } \\
3 / 227 \text { ton }\end{array}$ & 2035 & 3.505 & 2.697 & 472.8 & 388.8 & 308.077 & 393.0 & 1.192 & 0.909 & 2.51 & 4.58 & 88.04 & 4.57 & 87.84 & 17.77 & 54.84 & 23.05 & 10.7 \\
\hline & 2036 & 3.506 & 2.690 & 488.0 & 400.9 & 317.521 & 403.9 & 1.228 & 0.927 & 2.51 & 4.64 & 89.25 & 4.64 & 89.27 & 17.85 & 55.55 & 23.27 & 10.7 \\
\hline & 2037 & 3.506 & 2.707 & 488.4 & 401.8 & 317.549 & 405.8 & 1.226 & 0.940 & 2.52 & 4.55 & 87.56 & 4.53 & 87.13 & 17.73 & 54.28 & 22.79 & 10.7 \\
\hline & 2039 & 3.508 & 2.683 & 483.0 & 399.1 & N/A & N/A & 1.220 & 0.924 & 2.50 & N/A & N/A & 4.66 & 89.63 & 17.37 & 55.69 & 23.52 & 10.7 \\
\hline GA-25-10\% & 1634 & 3.252 & 2.545 & 499.5 & 445.4 & 353.327 & 445.7 & 1.426 & 1.116 & 2.57 & 4.82 & 92.73 & 4.79 & 92.04 & 10.83 & 52.06 & 21.74 & 6.1 \\
\hline GA-25-14\% & 1635 & 3.253 & 2.593 & 499.2 & 435.1 & 345.317 & 436.1 & 1.315 & 1.060 & 2.79 & 4.79 & 92.17 & 4.74 & 91.19 & 12.84 & 48.77 & 20.29 & 8.4 \\
\hline GA-26-10\% & 1655 & 3.255 & 2.575 & 499.5 & 449.7 & 357.061 & 451.7 & 1.420 & 1.125 & 2.58 & 4.75 & 91.38 & 4.68 & 90.05 & 9.97 & 50.41 & 20.89 & 5.5 \\
\hline GA-26-14\% & 1653 & 3.253 & 2.609 & 499.9 & 414.6 & 328.320 & 414.8 & 1.256 & 1.000 & 2.92 & 4.79 & 92.20 & 4.73 & 90.98 & 17.06 & 48.78 & 19.80 & 11.4 \\
\hline $\begin{array}{l}\text { GA-26-14\% } \\
\text { (repeat) }\end{array}$ & 1654 & 3.254 & 2.615 & 499.2 & 438.7 & 348.247 & 440.1 & 1.310 & 1.054 & 2.80 & 4.78 & 91.85 & 4.73 & 90.92 & 12.12 & 48.03 & 19.64 & 8.4 \\
\hline GA-28-10\% CP & 1706 & 3.256 & 2.565 & 497.9 & 433.6 & 343.605 & 434.1 & 1.369 & 1.072 & 2.67 & 4.79 & 92.14 & 4.78 & 91.83 & 12.91 & 51.40 & 21.22 & 7.1 \\
\hline $\begin{array}{l}\text { GA-28-10\% BP } \\
4 / 77.5 \text { ton }\end{array}$ & 2090 & 3.502 & 2.768 & 508.9 & 443.2 & 350.762 & 443.5 & 1.199 & 0.942 & 2.69 & 4.78 & 91.90 & 4.77 & 91.73 & 12.91 & 50.91 & 20.96 & 6.6 \\
\hline & 2091 & 3.505 & 2.761 & 482.2 & 421.6 & 332.899 & 422.0 & 1.160 & 0.908 & 2.63 & 4.73 & 90.99 & 4.73 & 90.98 & 12.57 & 51.42 & 21.23 & 6.6 \\
\hline 8 ton & 2092 & 3.505 & 2.751 & 498.0 & 433.6 & 343.664 & 433.8 & 1.193 & 0.925 & 2.64 & 4.81 & 92.51 & 4.81 & 92.52 & 12.93 & 52.23 & 21.51 & 6.6 \\
\hline 10 ton & 2093 & 3.506 & 2.761 & 488.0 & 424.1 & 336.372 & 424.0 & 1.146 & 0.908 & 2.69 & 4.84 & 93.07 & 4.76 & 91.52 & 13.09 & 50.85 & 21.25 & 6.6 \\
\hline 13.5 ton & 2094 & 3.506 & 2.785 & 502.2 & 436.4 & 346.479 & 436.4 & 1.143 & 0.900 & 2.78 & 4.85 & 93.33 & 4.86 & 93.38 & 13.10 & 50.31 & 20.56 & 6.6 \\
\hline & 2095 & 3.506 & 2.780 & 499.9 & 434.1 & 345.066 & 434.4 & 1.131 & 0.896 & 2.79 & 4.86 & 93.45 & 4.87 & 93.64 & 13.16 & 50.18 & 20.71 & 6.6 \\
\hline $\begin{array}{l}\text { GA-28-12\% CP } \\
\text { G0210-2 }\end{array}$ & 1707 & 3.257 & 2.499 & 500.1 & 418.4 & 331.565 & 418.9 & 1.437 & 1.092 & 2.55 & 4.79 & 92.13 & 4.77 & 91.64 & 16.34 & 55.26 & 23.27 & 9.2 \\
\hline & 1772 & 3.255 & 2.546 & 500.6 & 432.7 & 342.839 & 434.2 & 1.410 & 1.113 & 2.60 & 4.74 & 91.08 & 4.66 & 89.59 & 13.56 & 51.70 & 21.78 & 8.0 \\
\hline $\begin{array}{l}\text { GA-28-12\% } \\
\text { G0210-2 BP } \\
\text { 4/12.7 ton } \\
\end{array}$ & 2098 & 3.507 & 2.727 & 455.5 & 396.1 & 313.971 & 396.3 & 1.110 & 0.868 & 2.59 & 4.81 & 92.52 & 4.77 & 91.66 & 13.04 & 52.71 & 22.24 & 9.2 \\
\hline 8 ton & 2099 & 3.507 & 2.668 & 437.8 & 369.3 & 293.950 & 370.0 & 1.093 & 0.831 & 2.53 & 4.86 & 93.38 & 4.85 & 93.26 & 15.65 & 55.99 & 23.92 & 9.2 \\
\hline 7.5 ton & 2100 & 3.507 & 2.671 & 475.5 & 397.1 & 315.747 & 397.3 & 1.175 & 0.897 & 2.56 & 4.87 & 93.64 & 4.82 & 92.69 & 16.49 & 55.71 & 23.84 & 9.2 \\
\hline 7 ton & 2101 & 3.507 & 2.680 & 495.6 & 414.3 & 328.980 & 414.6 & 1.232 & 0.941 & 2.54 & 4.84 & 93.05 & 4.76 & 91.56 & 16.40 & 55.39 & 23.58 & 9.2 \\
\hline
\end{tabular}

Page 26 of 49 
Appendix F - Puck Data for Granulation Matrix Testing

\begin{tabular}{|c|c|c|c|c|c|c|c|c|c|c|c|c|c|c|c|c|c|c|}
\hline Batch ID & $\begin{array}{c}\text { Puck } \\
\text { ID }\end{array}$ & $\begin{array}{c}\text { Green } \\
\text { Puck } \\
\text { Diam. } \\
\text { (in.) }\end{array}$ & $\begin{array}{l}\text { Sintered } \\
\text { Puck } \\
\text { Diameter } \\
\text { (in.) }\end{array}$ & $\begin{array}{c}\text { Green } \\
\text { Weight } \\
\text { (g) }\end{array}$ & $\begin{array}{c}\text { Sintered } \\
\text { Puck } \\
\text { Weight } \\
\text { (g) }\end{array}$ & $\begin{array}{c}\quad \text { Puck } \\
\text { Suspended } \\
\text { Weight }(\mathrm{g})\end{array}$ & $\begin{array}{c}\text { Wet } \\
\text { Weight } \\
\text { (g) }\end{array}$ & $\begin{array}{c}\text { Green } \\
\text { Puck } \\
\text { Thick. } \\
\text { (in.) }\end{array}$ & \begin{tabular}{|c|} 
Sintered \\
Puck \\
Thick. \\
(in.)
\end{tabular} & $\begin{array}{c}\text { Green } \\
\text { Density } \\
\text { (g/cc) }\end{array}$ & $\begin{array}{c}\text { Archi- } \\
\text { medes } \\
\text { Density } \\
(\mathrm{g} / \mathrm{cc})\end{array}$ & $\begin{array}{c}\text { Archi- } \\
\text { medes } \\
\text { Density } \\
(\% \text { TD) }\end{array}$ & $\begin{array}{c}\text { Geom. } \\
\text { Density } \\
\text { (g/cc) }\end{array}$ & $\begin{array}{l}\text { Geom. } \\
\text { (\% TD) }\end{array}$ & $\begin{array}{c}\text { Mass \% } \\
\text { Shrink- } \\
\text { age }\end{array}$ & $\begin{array}{c}\text { Volume } \\
\% \\
\text { Shinkage }\end{array}$ & \begin{tabular}{|c|} 
Diam. \\
$\%$ \\
Shrink- \\
age
\end{tabular} & $\begin{array}{l}\text { Powder } \\
\% \text { Moist }\end{array}$ \\
\hline $\begin{array}{l}\text { GA-28-12\% Full } \\
\text { G0214-0 CP }\end{array}$ & 1749 & 3.255 & 2.526 & 500.3 & 430.5 & 341.639 & 430.5 & 1.415 & 1.095 & 2.59 & 4.84 & 93.17 & 4.79 & 92.04 & 13.95 & 53.39 & 22.40 & 8.0 \\
\hline $\begin{array}{l}\text { GA-28-12\%, } \\
\text { G0214-0 Full, } 10 \\
\text { ton BP 4/12 }\end{array}$ & 2119 & 3.507 & 2.713 & 494.3 & 424.3 & 336.868 & 424.7 & 1.218 & 0.943 & 2.56 & 4.83 & 92.90 & 4.75 & 91.31 & 14.16 & 53.66 & 22.64 & 8.0 \\
\hline 7.5 ton & 2120 & 3.507 & 2.707 & 493.9 & 424.0 & 337.422 & 424.3 & 1.210 & 0.941 & 2.58 & 4.88 & 93.85 & 4.78 & 91.85 & 14.15 & 53.66 & 22.81 & 8.0 \\
\hline 8 ton & 2121 & 3.507 & 2.710 & 495.3 & 425.7 & 337.897 & 426.0 & 1.212 & 0.934 & 2.58 & 4.83 & 92.92 & 4.82 & 92.70 & 14.05 & 53.98 & 22.73 & 8.0 \\
\hline 10 ton & 2122 & 3.507 & 2.717 & 486.6 & 418.3 & 332.963 & 418.6 & 1.171 & 0.908 & 2.62 & 4.88 & 93.93 & 4.85 & 93.22 & 14.04 & 53.45 & 22.53 & 8.0 \\
\hline & 2123 & 3.507 & 2.732 & 492.2 & 423.1 & 335.755 & 423.7 & 1.188 & 0.922 & 2.62 & 4.81 & 92.52 & 4.78 & 91.84 & 14.04 & 52.89 & 22.10 & 8.0 \\
\hline 8 ton & 2126 & 3.505 & 2.729 & 484.6 & 417.2 & 329.906 & 417.7 & 1.190 & 0.931 & 2.58 & 4.75 & 91.39 & 4.67 & 89.88 & 13.91 & 52.56 & 22.14 & 8.0 \\
\hline \begin{tabular}{|l|} 
GA-28-12\%, BP \\
4/12, G0316-0 \\
Full, 9 ton
\end{tabular} & 2105 & 3.507 & 2.734 & 584.8 & 490.5 & 387.394 & 491.1 & 1.397 & 1.084 & 2.64 & 4.73 & 90.96 & 4.70 & 90.42 & 16.13 & 52.83 & 22.04 & 9.7 \\
\hline 10 ton & 2106 & 3.507 & 2.730 & 527.1 & 441.6 & 350.280 & 442.0 & 1.243 & 0.974 & 2.68 & 4.81 & 92.59 & 4.73 & 90.87 & 16.22 & 52.51 & 22.16 & 9.7 \\
\hline 15 ton & 2107 & 3.508 & 2.758 & 507.6 & 425.4 & 338.350 & 425.7 & 1.143 & 0.909 & 2.80 & 4.87 & 93.66 & 4.78 & 91.90 & 16.19 & 50.83 & 21.38 & 9.7 \\
\hline 20 ton & 2108 & 3.509 & 2.775 & 510.2 & 427.7 & 340.390 & 428.1 & 1.126 & 0.895 & 2.86 & 4.88 & 93.77 & 4.82 & 92.70 & 16.17 & 50.28 & 20.92 & 9.7 \\
\hline 10 ton & 2110 & 3.508 & 2.741 & 505.1 & 423.2 & 334.279 & 423.6 & 1.190 & 0.940 & 2.68 & 4.74 & 91.11 & 4.65 & 89.51 & 16.21 & 51.77 & 21.86 & 9.7 \\
\hline & 2111 & 3.508 & 2.742 & 506.0 & 423.8 & 334.820 & 424.2 & 1.196 & 0.936 & 2.67 & 4.74 & 91.18 & 4.68 & 89.95 & 16.25 & 52.18 & 21.84 & 9.7 \\
\hline & 2113 & 3.508 & 2.748 & 507.9 & 425.5 & 335.625 & 426.0 & 1.203 & 0.944 & 2.67 & 4.71 & 90.54 & 4.64 & 89.16 & 16.22 & 51.84 & 21.66 & 9.7 \\
\hline \begin{tabular}{|l|} 
GA-28-14\%, CP \\
G0210-1
\end{tabular} & 1705 & 3.255 & 2.544 & 499.0 & 416.0 & 331.050 & 416.6 & 1.330 & 1.038 & 2.75 & 4.86 & 93.51 & 4.81 & 92.50 & 16.63 & 52.32 & 21.84 & 9.8 \\
\hline \begin{tabular}{|l|}
$\mathrm{GA}-28-14 \%, \mathrm{BP}$ \\
$4 / 12, \mathrm{G} 0210-1$, \\
10 ton
\end{tabular} & 2128 & 3.505 & 2.845 & 505.3 & 430.2 & N/A & N/A & 1.194 & 0.933 & 2.68 & N/A & N/A & 4.42 & 85.09 & 14.86 & 48.51 & 18.83 & 9.8 \\
\hline & 2129 & 3.505 & 2.729 & 551.8 & 460.9 & 367.327 & 461.4 & 1.262 & 0.983 & 2.77 & 4.90 & 94.22 & 4.89 & 94.04 & 16.47 & 52.77 & 22.14 & 9.8 \\
\hline & 2130 & 3.505 & 2.736 & 527.1 & 438.8 & 349.340 & 439.4 & 1.196 & 0.947 & 2.79 & 4.87 & 93.70 & 4.81 & 92.46 & 16.75 & 51.74 & 21.94 & 9.8 \\
\hline & 2131 & 3.505 & 2.731 & 503.2 & 418.0 & 333.570 & 415.6 & 1.143 & 0.890 & 2.78 & 5.10 & 97.99 & 4.89 & 94.06 & 16.93 & 52.72 & 22.08 & 9.8 \\
\hline & 2133 & 3.507 & 2.729 & 472.0 & 391.4 & 312.708 & 392.8 & 1.073 & 0.849 & 2.78 & 4.89 & 93.98 & 4.81 & 92.47 & 17.08 & 52.08 & 22.18 & 9.8 \\
\hline $\begin{array}{l}\text { GA-28-14\% CP } \\
\text { Full. G0214-1 }\end{array}$ & 1750 & 3.255 & 2.534 & 499.3 & 411.9 & 326.292 & 411.9 & 1.360 & 1.050 & 2.69 & 4.81 & 92.53 & 4.75 & 91.26 & 17.50 & 53.20 & 22.15 & 11.1 \\
\hline $\begin{array}{l}\text { GA-28-14\%, } \\
\text { G0214-1 Full BP } \\
4 / 12.10 \text { ton }\end{array}$ & 2134 & 3.507 & 2.720 & 435.9 & 359.5 & 286.818 & 360.6 & 1.009 & 0.793 & 2.73 & 4.87 & 93.70 & 4.76 & 91.53 & 17.53 & 52.71 & 22.44 & 11.1 \\
\hline & 2135 & 3.507 & 2.718 & 476.1 & 391.9 & 311.928 & 392.0 & 1.097 & 0.855 & 2.74 & 4.89 & 94.12 & 4.82 & 92.68 & 17.69 & 53.18 & 22.50 & 11.1 \\
\hline & 2137 & 3.507 & 2.725 & 497.4 & 409.3 & 325.046 & 409.3 & 1.143 & 0.889 & 2.75 & 4.86 & 93.42 & 4.82 & 92.61 & 17.71 & 53.03 & 22.30 & 11.1 \\
\hline & 2138 & 3.507 & 2.756 & 493.8 & 406.5 & 321.940 & 406.5 & 1.136 & 0.889 & 2.75 & 4.81 & 92.45 & 4.68 & 89.92 & 17.68 & 51.66 & 21.41 & 11.1 \\
\hline & 2141 & 3.507 & 2.730 & 485.0 & 397.1 & 317.632 & 398.5 & 1.092 & 0.855 & 2.81 & 4.91 & 94.43 & 4.84 & 93.09 & 18.12 & 52.55 & 22.16 & 11.1 \\
\hline & 2142 & 3.507 & 2.720 & 505.6 & 414.2 & 330.486 & 415.5 & 1.159 & 0.897 & 2.76 & 4.87 & 93.69 & 4.85 & 93.23 & 18.08 & 53.44 & 22.44 & 11.1 \\
\hline & 2143 & 3.507 & 2.717 & 505.3 & 415.0 & 330.454 & 415.0 & 1.158 & 0.896 & 2.76 & 4.91 & 94.40 & 4.87 & 93.72 & 17.87 & 53.55 & 22.53 & 11.1 \\
\hline \begin{tabular}{|l|} 
GA-28-14\% Full \\
CP $(\mathrm{G} 0315-1)$
\end{tabular} & 1942 & 3.256 & 2.543 & 499.4 & 415.6 & 329.758 & 415.6 & 1.326 & 1.032 & 2.76 & 4.84 & 93.10 & 4.84 & 93.02 & 16.78 & 52.52 & 21.90 & 10.3 \\
\hline
\end{tabular}

Page 27 of 49 
Appendix F - Puck Data for Granulation Matrix Testing

\begin{tabular}{|c|c|c|c|c|c|c|c|c|c|c|c|c|c|c|c|c|c|c|}
\hline Batch ID & $\begin{array}{c}\text { Puck } \\
\text { ID }\end{array}$ & $\begin{array}{c}\text { Green } \\
\text { Puck } \\
\text { Diam. } \\
\text { (in.) }\end{array}$ & $\begin{array}{c}\text { Sintered } \\
\text { Puck } \\
\text { Diameter } \\
\text { (in.) }\end{array}$ & $\begin{array}{l}\text { Green } \\
\text { Weight } \\
\text { (g) }\end{array}$ & $\begin{array}{l}\text { Sintered } \\
\text { Puck } \\
\text { Weight } \\
\text { (g) }\end{array}$ & $\begin{array}{c}\text { Puck } \\
\text { Suspended } \\
\text { Weight }(g)\end{array}$ & $\begin{array}{c}\text { Wet } \\
\text { Weight } \\
\text { (g) }\end{array}$ & $\begin{array}{c}\text { Green } \\
\text { Puck } \\
\text { Thick. } \\
\text { (in.) }\end{array}$ & \begin{tabular}{|c|} 
Sintered \\
Puck \\
Thick. \\
(in.)
\end{tabular} & $\begin{array}{c}\text { Green } \\
\text { Density } \\
\text { (g/cc) }\end{array}$ & $\begin{array}{c}\text { Archi- } \\
\text { medes } \\
\text { Density } \\
\text { (g/cc) }\end{array}$ & $\begin{array}{c}\text { Archi- } \\
\text { medes } \\
\text { Density } \\
(\% \mathrm{TD})\end{array}$ & $\begin{array}{c}\text { Geom. } \\
\text { Density } \\
\text { (g/cc) }\end{array}$ & $\begin{array}{l}\text { Geom. } \\
\text { (\% TD) }\end{array}$ & $\begin{array}{c}\text { Mass \% } \\
\text { Shrink- } \\
\text { age }\end{array}$ & $\begin{array}{c}\text { Volume } \\
\% \\
\text { Shinkage }\end{array}$ & \begin{tabular}{|c|} 
Diam. \\
$\%$ \\
Shrink- \\
age
\end{tabular} & $\begin{array}{l}\text { Powder } \\
\% \text { Moist }\end{array}$ \\
\hline $\begin{array}{l}\text { GA-28-14\% } \\
\text { Full, BP 4/12, 10 } \\
\text { ton. G0315-1 }\end{array}$ & 2148 & 3.507 & 2.737 & 520.4 & 430.6 & 343.328 & 431.0 & 1.186 & 0.920 & 2.77 & 4.91 & 94.45 & 4.85 & 93.33 & 17.26 & 52.74 & 21.96 & 10.3 \\
\hline 7 ton & $\begin{array}{l}2154 \\
2155\end{array}$ & $\begin{array}{l}3.506 \\
3.506\end{array}$ & $\frac{2.713}{2.705}$ & $\begin{array}{l}498.6 \\
489.2\end{array}$ & $\begin{array}{l}412.8 \\
404.9\end{array}$ & $\begin{array}{l}327.951 \\
322.550\end{array}$ & $\begin{array}{l}413.0 \\
405.1\end{array}$ & \begin{tabular}{|l|}
1.170 \\
1.158 \\
\end{tabular} & $\begin{array}{l}0.915 \\
0.894\end{array}$ & $\begin{array}{l}2.69 \\
2.67\end{array}$ & $\begin{array}{l}4.85 \\
4.90\end{array}$ & $\begin{array}{l}93.34 \\
94.33\end{array}$ & $\begin{array}{l}4.76 \\
4.81\end{array}$ & $\begin{array}{l}91.56 \\
92.46\end{array}$ & $\begin{array}{l}17.21 \\
17.23\end{array}$ & $\begin{array}{l}53.16 \\
54.04\end{array}$ & $\begin{array}{l}22.62 \\
22.85\end{array}$ & $\begin{array}{l}10.3 \\
10.3\end{array}$ \\
\hline $\begin{array}{l}\text { GA-28-14\% CP } \\
\text { Full (G0315-2) } \\
\end{array}$ & 1940 & 3.253 & 2.591 & 499.6 & 412.1 & 328.386 & 412.1 & 1.237 & 0.983 & 2.97 & 4.92 & 94.67 & 4.85 & 93.28 & 17.51 & 49.58 & 20.35 & 11.2 \\
\hline $\begin{array}{l}\text { GA-28-14\% } \\
\text { Full, BP 4/12, } 7 \\
\text { ton. G0315-2 }\end{array}$ & 2159 & 3.512 & 2.783 & 508.2 & 416.6 & 333.309 & 418.1 & 1.110 & 0.879 & 2.88 & 4.91 & 94.49 & 4.75 & 91.41 & 18.02 & 50.26 & 20.76 & 11.2 \\
\hline 5 ton & 2162 & 3.506 & 2.770 & 523.4 & 429.0 & 342.537 & 430.4 & 1.146 & 0.909 & 2.89 & 4.88 & 93.90 & 4.78 & 91.88 & 18.04 & 50.48 & 20.99 & 11.2 \\
\hline 4 ton & 2163 & 3.504 & 2.778 & 523.1 & 429.9 & 341.649 & 431.9 & 1.161 & 0.914 & 2.85 & 4.76 & 91.60 & 4.73 & 91.04 & 17.82 & 50.51 & 20.72 & 11.2 \\
\hline & 2164 & 3.503 & 2.759 & 484.8 & 396.6 & 316.049 & 398.4 & 1.089 & 0.853 & 2.82 & 4.82 & 92.61 & 4.74 & 91.24 & 18.19 & 51.40 & 21.24 & 11.2 \\
\hline & 2166 & 3.503 & 2.761 & 480.0 & 393.3 & 312.606 & 394.4 & 1.075 & 0.849 & 2.83 & 4.81 & 92.47 & 4.72 & 90.77 & 18.06 & 50.93 & 21.18 & 11.2 \\
\hline & 2167 & 3.504 & 2.774 & 492.0 & 403.3 & 320.502 & 404.9 & 1.093 & 0.868 & 2.85 & 4.78 & 91.90 & 4.69 & 90.19 & 18.03 & 50.22 & 20.83 & 11.2 \\
\hline $\begin{array}{l}\text { GA-28-14\%, } \\
\text { G0331-0, BP } \\
7 / 7.10 .6 \text { tons } \\
\end{array}$ & 2776 & 3.507 & 2.780 & 519.6 & 450.5 & 359.924 & 450.8 & 1.162 & 0.923 & 2.82 & 4.96 & 95.33 & 4.91 & 94.34 & 13.30 & 50.08 & 20.73 & 8.7 \\
\hline 5.75 tons & 2777 & 3.505 & 2.740 & 492.2 & 425.8 & 339.958 & 425.9 & 1.140 & 0.899 & 2.73 & 4.95 & 95.28 & 4.90 & 94.24 & 13.49 & 51.80 & 21.83 & 8.7 \\
\hline 5.8 tons & 2778 & 3.505 & 2.755 & 493.3 & 426.0 & 339.972 & 426.2 & 1.155 & 0.910 & 2.70 & 4.94 & 95.01 & 4.79 & 92.13 & 13.64 & 51.31 & 21.40 & 8.7 \\
\hline & 2779 & 3.506 & 2.739 & 492.0 & 425.0 & 338.808 & 425.4 & 1.143 & 0.908 & 2.72 & 4.91 & 94.39 & 4.85 & 93.19 & 13.62 & 51.51 & 21.88 & 8.7 \\
\hline & 2780 & 3.506 & 2.741 & 495.3 & 427.5 & 341.770 & 427.6 & 1.147 & 0.900 & 2.73 & 4.98 & 95.78 & 4.91 & 94.44 & 13.69 & 52.03 & 21.82 & 8.7 \\
\hline 6 tons & 2781 & 3.505 & 2.747 & 500.0 & 431.8 & 345.003 & 432.0 & 1.150 & 0.912 & 2.75 & 4.96 & 95.45 & 4.87 & 93.72 & 13.64 & 51.28 & 21.63 & 8.7 \\
\hline $\begin{array}{l}\text { GA-28-14\%, } \\
\text { G0331-1, BP } \\
7 / 7.6 .2 \text { tons }\end{array}$ & 2782 & 3.505 & 2.748 & 501.0 & 431.5 & 344.948 & 431.7 & 1.146 & 0.904 & 2.76 & 4.97 & 95.65 & 4.91 & 94.42 & 13.87 & 51.50 & 21.60 & 9.9 \\
\hline 3.8 tons & 2783 & 3.505 & 2.711 & 478.7 & 408.8 & 325.960 & 408.9 & 1.139 & 0.893 & 2.66 & 4.93 & 94.79 & 4.84 & 93.04 & 14.60 & 53.09 & 22.65 & 9.9 \\
\hline 6.4 tons & 2784 & 3.505 & 2.750 & 506.2 & 433.8 & 346.663 & 434.0 & 1.155 & 0.916 & 2.77 & 4.97 & 95.52 & 4.86 & 93.54 & 14.30 & 51.17 & 21.54 & 9.9 \\
\hline & 2785 & 3.505 & 2.748 & 506.4 & 433.2 & 346.553 & 433.4 & 1.153 & 0.912 & 2.78 & 4.99 & 95.92 & 4.89 & 93.96 & 14.45 & 51.37 & 21.60 & 9.9 \\
\hline 6.5 tons & 2786 & 3.506 & 2.749 & 508.3 & 435.7 & 348.342 & 436.0 & 1.150 & 0.915 & 2.79 & 4.97 & 95.59 & 4.89 & 94.12 & 14.28 & 51.08 & 21.59 & 9.9 \\
\hline ? Tons & 2787 & 3.505 & 2.751 & 509.2 & 434.0 & 347.153 & 434.3 & 1.150 & 0.914 & 2.80 & 4.98 & 95.77 & 4.87 & 93.72 & 14.77 & 51.03 & 21.51 & 9.9 \\
\hline 1.8 tons & 2788 & 3.505 & 2.660 & 438.4 & 375.7 & 298.338 & 376.4 & 1.135 & 0.871 & 2.44 & 4.81 & 92.55 & 4.74 & 91.06 & 14.30 & 55.79 & 24.11 & 9.9 \\
\hline GA-29-8\% & 1636 & 3.255 & 2.599 & 498.6 & 440.1 & 350.645 & 440.4 & 1.304 & 1.030 & 2.80 & 4.90 & 94.30 & 4.91 & 94.49 & 11.73 & 49.63 & 20.15 & 7.7 \\
\hline GA-29-10\% & 1637 & 3.252 & 2.622 & 499.2 & 431.5 & 343.564 & 431.6 & 1.253 & 1.007 & 2.93 & 4.90 & 94.26 & 4.84 & 93.10 & 13.56 & 47.75 & 19.37 & 9.0 \\
\hline $\begin{array}{l}\text { GA-29-10\% } \\
\text { Repeat }\end{array}$ & 1647 & 3.253 & 2.644 & 499.4 & 432.1 & 342.338 & 432.4 & 1.242 & 1.006 & 2.95 & 4.80 & 92.27 & 4.77 & 91.78 & 13.48 & 46.48 & 18.72 & 9.2 \\
\hline $\begin{array}{l}\text { GA-29-10\%, BP } \\
4 / 12,10 \text { ton }\end{array}$ & 2170 & 3.513 & 2.798 & 540.5 & 447.9 & 358.037 & 448.4 & 1.152 & 0.911 & 2.95 & 4.96 & 95.32 & 4.88 & 93.81 & 17.13 & 49.83 & 20.35 & 9.2 \\
\hline & 2172 & 3.503 & 2.813 & 533.5 & 459.7 & 365.955 & 460.0 & 1.161 & 0.925 & 2.91 & 4.89 & 94.00 & 4.88 & 93.81 & 13.83 & 48.61 & 19.70 & 9.2 \\
\hline 7 ton & 2173 & 3.504 & 2.789 & 509.8 & 439.1 & 348.583 & 439.4 & 1.143 & 0.905 & 2.82 & 4.83 & 92.98 & 4.84 & 93.17 & 13.87 & 49.83 & 20.41 & 9.2 \\
\hline & 2174 & 3.503 & 2.789 & 507.6 & 436.7 & 346.820 & 436.8 & 1.130 & 0.908 & 2.84 & 4.85 & 93.33 & 4.80 & 92.36 & 13.97 & 49.05 & 20.38 & 9.2 \\
\hline & 2177 & 3.506 & 2.811 & 496.9 & 430.2 & 339.107 & 430.2 & 1.115 & 0.891 & 2.82 & 4.72 & 90.82 & 4.75 & 91.27 & 13.42 & 48.62 & 19.82 & 9.2 \\
\hline & 2178 & 3.503 & 2.811 & 493.1 & 426.0 & 336.423 & 426.0 & 1.100 & 0.883 & 2.84 & 4.76 & 91.46 & 4.74 & 91.20 & 13.61 & 48.30 & 19.75 & 9.2 \\
\hline & 2179 & 3.504 & 2.811 & 492.2 & 424.3 & 335.550 & 424.3 & 1.097 & 0.880 & 2.84 & 4.78 & 91.94 & 4.74 & 91.15 & 13.80 & 48.36 & 19.78 & 9.2 \\
\hline GA-30-8\% CP & 1646 & 3.255 & 2.592 & 499.5 & 441.8 & 351.409 & 442.3 & 1.312 & 1.055 & 2.79 & 4.86 & 93.48 & 4.84 & 93.11 & 11.55 & 49.00 & 20.37 & 6.9 \\
\hline
\end{tabular}

Page 28 of 49 
Appendix F - Puck Data for Granulation Matrix Testing

\begin{tabular}{|c|c|c|c|c|c|c|c|c|c|c|c|c|c|c|c|c|c|c|}
\hline Batch ID & $\begin{array}{c}\text { Puck } \\
\text { ID }\end{array}$ & $\begin{array}{c}\text { Green } \\
\text { Puck } \\
\text { Diam. } \\
\text { (in.) }\end{array}$ & $\begin{array}{c}\text { Sintered } \\
\text { Puck } \\
\text { Diameter } \\
\text { (in.) }\end{array}$ & $\begin{array}{c}\text { Green } \\
\text { Weight } \\
\text { (g) }\end{array}$ & \begin{tabular}{|c} 
Sintered \\
Puck \\
Weight \\
(g)
\end{tabular} & $\begin{array}{c}\text { Puck } \\
\text { Suspended } \\
\text { Weight (g) }\end{array}$ & $\begin{array}{c}\text { Wet } \\
\text { Weight } \\
\text { (g) }\end{array}$ & $\begin{array}{c}\text { Green } \\
\text { Puck } \\
\text { Thick. } \\
\text { (in.) }\end{array}$ & \begin{tabular}{|c|} 
Sintered \\
Puck \\
Thick. \\
(in.)
\end{tabular} & $\begin{array}{c}\text { Green } \\
\text { Density } \\
\text { (g/cc) }\end{array}$ & $\begin{array}{c}\text { Archi- } \\
\text { medes } \\
\text { Density } \\
\text { (g/cc) }\end{array}$ & $\begin{array}{c}\text { Archi- } \\
\text { medes } \\
\text { Density } \\
(\% \mathrm{TD})\end{array}$ & $\begin{array}{c}\text { Geom. } \\
\text { Density } \\
\text { (g/cc) }\end{array}$ & $\begin{array}{l}\text { Geom. } \\
\text { (\% TD) }\end{array}$ & $\begin{array}{c}\text { Mass \% } \\
\text { Shrink- } \\
\text { age }\end{array}$ & $\begin{array}{c}\text { Volume } \\
\% \\
\text { Shinkage }\end{array}$ & \begin{tabular}{|c|} 
Diam. \\
$\%$ \\
Shrink- \\
age
\end{tabular} & $\begin{array}{l}\text { Powder } \\
\text { \% Moist }\end{array}$ \\
\hline $\begin{array}{l}\text { GA-30-8\%,BP } \\
6 / 19,23 \text { tons }\end{array}$ & 2611 & 3.511 & 2.851 & 578.2 & 512.0 & 410.390 & 512.8 & 1.200 & 0.991 & 3.04 & 5.00 & 96.14 & 4.94 & 94.95 & 11.45 & 45.54 & 18.80 & 6.9 \\
\hline 13.1 tons & 2612 & 3.507 & 2.801 & 529.2 & 466.1 & 372.585 & 466.0 & 1.163 & 0.929 & 2.87 & 4.99 & 95.95 & 4.97 & 95.52 & 11.92 & 49.04 & 20.13 & 6.9 \\
\hline 6.3 tons & 2613 & 3.505 & 2.749 & 479.0 & 421.6 & 334.649 & 421.6 & 1.145 & 0.903 & 2.65 & 4.85 & 93.24 & 4.80 & 92.29 & 11.98 & 51.48 & 21.57 & 6.9 \\
\hline 8.1 tons & 2614 & 3.506 & 2.782 & 508.9 & 444.4 & 354.058 & 444.5 & 1.153 & 0.915 & 2.79 & 4.91 & 94.49 & 4.87 & 93.74 & 12.67 & 50.02 & 20.65 & 6.9 \\
\hline 6.4 tons & 2615 & 3.505 & 2.768 & 511.9 & 443.7 & 352.315 & 443.7 & 1.174 & 0.930 & 2.76 & 4.86 & 93.37 & 4.84 & 93.01 & 13.32 & 50.59 & 21.03 & 6.9 \\
\hline GA-30-10\% CP & 1643 & 3.253 & 2.614 & 499.2 & 432.8 & 343.475 & 432.8 & 1.262 & 1.021 & 2.90 & 4.85 & 93.18 & 4.82 & 92.67 & 13.30 & 47.75 & 19.64 & 8.3 \\
\hline & 2070 & 3.253 & 2.567 & 499.5 & 437.0 & 348.668 & 437.1 & 1.313 & 1.047 & 2.79 & 4.94 & 95.03 & 4.92 & 94.61 & 12.51 & 50.34 & 21.09 & 8.0 \\
\hline $\begin{array}{l}\text { GA-30-10\% BP } \\
4 / 3,7 \text { ton, } \\
\text { G0202-2 \& } \\
\text { G0223-2 }\end{array}$ & 2071 & 3.498 & & 475.0 & & & & 1.189 & & & & & & & & & & \\
\hline & 2072 & 3.502 & 2.762 & 499.4 & 435.5 & 344.268 & 435.5 & 1.195 & 0.942 & 2.65 & 4.77 & 91.80 & 4.71 & 90.52 & 12.80 & 50.96 & 21.13 & 8.0 \\
\hline & 2073 & 3.501 & 2.757 & 507.9 & 441.1 & 349.894 & 441.2 & 1.200 & 0.950 & 2.68 & 4.83 & 92.90 & 4.74 & 91.25 & 13.15 & 50.90 & 21.25 & 8.0 \\
\hline & 2074 & 3.503 & 2.746 & 497.3 & 431.4 & 343.103 & 431.4 & 1.162 & 0.912 & 2.71 & 4.89 & 93.96 & 4.87 & 93.70 & 13.25 & 51.76 & 21.61 & 8.0 \\
\hline & 2075 & 3.502 & 2.772 & 502.8 & 436.9 & 345.408 & 437.1 & 1.182 & 0.943 & 2.69 & 4.76 & 91.63 & 4.68 & 90.06 & 13.11 & 50.00 & 20.85 & 8.0 \\
\hline & 2076 & 3.501 & 2.752 & 501.7 & 435.3 & 345.888 & 435.3 & 1.181 & 0.912 & 2.69 & 4.87 & 93.62 & 4.90 & 94.14 & 13.24 & 52.28 & 21.39 & 8.0 \\
\hline & 2077 & 3.502 & 2.756 & 500.9 & 434.4 & 344.584 & 434.5 & 1.178 & 0.929 & 2.69 & 4.83 & 92.91 & 4.78 & 91.96 & 13.28 & 51.15 & 21.30 & 8.0 \\
\hline & 2078 & 3.503 & 2.768 & 498.6 & 433.1 & 342.578 & 433.2 & 1.162 & 0.929 & 2.72 & 4.78 & 91.91 & 4.73 & 90.89 & 13.14 & 50.07 & 20.98 & 8.0 \\
\hline & 2079 & 3.500 & 2.733 & 448.6 & 388.8 & 309.848 & 389.0 & 1.060 & 0.830 & 2.68 & 4.91 & 94.46 & 4.87 & 93.68 & 13.33 & 52.25 & 21.91 & 8.0 \\
\hline $\begin{array}{l}\text { GA-30-10\% Full } \\
\text { G0223-2 }\end{array}$ & 1788 & 3.257 & 2.590 & 500.5 & 438.2 & 347.802 & 438.3 & 1.319 & 1.052 & 2.78 & 4.84 & 93.12 & 4.82 & 92.75 & 12.45 & 49.56 & 20.48 & 8.0 \\
\hline GA-30-12\% CP & 1644 & 3.253 & 2.621 & 501.3 & 430.7 & 342.086 & 431.6 & 1.249 & 1.018 & 2.95 & 4.81 & 92.53 & 4.78 & 92.00 & 14.08 & 47.08 & 19.43 & 9.5 \\
\hline $\begin{array}{l}\text { GA-30-12\%, BP } \\
6 / 1967 \text { tons }\end{array}$ & 2616 & 3.506 & 2.773 & 512.2 & 442.0 & 350.873 & 442.2 & 1.169 & 0.929 & 2.77 & 4.84 & 93.07 & 4.81 & 92.42 & 13.71 & 50.28 & 20.91 & 9.5 \\
\hline 6.3 tons & 2617 & 3.507 & 2.750 & 514.1 & 441.8 & 352.418 & 442.1 & 1.168 & 0.921 & 2.78 & 4.93 & 94.74 & 4.93 & 94.75 & 14.06 & 51.51 & 21.59 & 9.5 \\
\hline 6.1 tons & 2618 & 3.505 & 2.754 & 517.4 & 443.7 & 353.402 & 443.1 & 1.169 & 0.924 & 2.80 & 4.95 & 95.13 & 4.92 & 94.57 & 14.24 & 51.19 & 21.43 & 9.5 \\
\hline & 2619 & 3.505 & 2.755 & 518.1 & 442.8 & 352.788 & 443.0 & 1.168 & 0.925 & 2.81 & 4.91 & 94.39 & 4.90 & 94.21 & 14.53 & 51.06 & 21.40 & 9.5 \\
\hline 6.4 tons & 2620 & 3.505 & 2.759 & 519.9 & 444.6 & 353.949 & 444.6 & 1.171 & 0.928 & 2.81 & 4.90 & 94.32 & 4.89 & 94.01 & 14.48 & 50.89 & 21.28 & 9.5 \\
\hline GA-31-8\% CP & 1648 & 3.253 & 2.596 & 499.8 & 443.2 & 352.467 & 443.3 & 1.320 & 1.053 & 2.78 & 4.88 & 93.83 & 4.85 & 93.29 & 11.32 & 49.19 & 20.20 & 6.8 \\
\hline $\begin{array}{l}\text { GA-31-8\%, BP } \\
6 / 19,4.5 \text { tons }\end{array}$ & 2621 & 3.504 & 2.713 & 477.2 & 417.4 & 331.462 & 417.4 & 1.162 & 0.909 & 2.60 & 4.86 & 93.40 & 4.85 & 93.19 & 12.53 & 53.10 & 22.57 & 6.8 \\
\hline 5.9 tons & 2622 & 3.505 & 2.714 & 487.9 & 429.9 & 343.407 & 429.9 & 1.167 & 0.911 & 2.64 & 4.97 & 95.58 & 4.98 & 95.70 & 11.89 & 53.19 & 22.57 & 6.8 \\
\hline 7.2 tons & 2623 & 3.506 & 2.743 & 508.5 & 448.0 & 358.295 & 448.1 & 1.178 & 0.928 & 2.73 & 4.99 & 95.93 & 4.98 & 95.84 & 11.90 & 51.77 & 21.76 & 6.8 \\
\hline 6.2 tons & 2624 & 3.508 & 2.744 & 507.5 & 447.2 & 357.171 & 447.2 & 1.174 & 0.928 & 2.73 & 4.97 & 95.52 & 4.97 & 95.60 & 11.88 & 51.63 & 21.78 & 6.8 \\
\hline 6 tons & 2625 & 3.505 & 2.724 & 501.4 & 441.9 & 352.663 & 441.7 & 1.172 & 0.923 & 2.71 & 4.96 & 95.44 & 5.01 & 96.38 & 11.87 & 52.42 & 22.28 & 6.8 \\
\hline GA-31-10\% CP & 1767 & 3.253 & 2.578 & 499.9 & 433.1 & 345.784 & 433.2 & 1.303 & 1.036 & 2.82 & 4.95 & 95.28 & 4.89 & 93.96 & 13.36 & 50.05 & 20.75 & 9.2 \\
\hline \begin{tabular}{|l|} 
GA- $31-10 \%$, BP \\
$6 / 19,5.9$ tons
\end{tabular} & 2626 & 3.505 & 2.724 & 502.3 & 437.1 & 348.909 & 437.1 & 1.170 & 0.922 & 2.71 & 4.96 & 95.31 & 4.96 & 95.43 & 12.98 & 52.39 & 22.28 & 9.2 \\
\hline & 2627 & 3.505 & 2.722 & 505.0 & 436.0 & 348.134 & 435.5 & 1.172 & 0.913 & 2.72 & 4.99 & 95.97 & 5.01 & 96.27 & 13.66 & 53.01 & 22.34 & 9.2 \\
\hline 5.2 tons & 2628 & 3.505 & 2.715 & 499.7 & 430.8 & 343.974 & 430.6 & 1.169 & 0.914 & 2.70 & 4.97 & 95.64 & 4.97 & 95.51 & 13.79 & 53.08 & 22.54 & 9.2 \\
\hline 5 tons & 2629 & 3.505 & 2.710 & 497.3 & 428.4 & 342.380 & 428.5 & 1.168 & 0.911 & 2.69 & 4.97 & 95.66 & 4.97 & 95.65 & 13.85 & 53.36 & 22.68 & 9.2 \\
\hline 4.8 tons & 2630 & 3.505 & 2.707 & 495.6 & 426.8 & 341.131 & 427.1 & 1.173 & 0.912 & 2.67 & 4.96 & 95.47 & 4.96 & 95.39 & 13.88 & 53.62 & 22.77 & 9.2 \\
\hline GA-31-12\% CP & 1645 & 3.252 & 2.621 & 501.0 & 425.7 & 339.074 & 426.0 & 1.234 & 0.991 & 2.98 & 4.90 & 94.18 & 4.86 & 93.40 & 15.03 & 47.82 & 19.40 & 10.2 \\
\hline
\end{tabular}

Page 29 of 49 
Appendix F - Puck Data for Granulation Matrix Testing

\begin{tabular}{|c|c|c|c|c|c|c|c|c|c|c|c|c|c|c|c|c|c|c|}
\hline Batch ID & $\begin{array}{c}\text { Puck } \\
\text { ID }\end{array}$ & $\begin{array}{c}\text { Green } \\
\text { Puck } \\
\text { Diam. } \\
\text { (in.) } \\
\end{array}$ & $\begin{array}{l}\text { Sintered } \\
\text { Puck } \\
\text { Diameter } \\
\text { (in.) } \\
\end{array}$ & $\begin{array}{c}\text { Green } \\
\text { Weight } \\
(\mathrm{g})\end{array}$ & $\begin{array}{c}\text { Sintered } \\
\text { Puck } \\
\text { Weight } \\
(\mathrm{g}) \\
\end{array}$ & $\begin{array}{c}\text { Puck } \\
\text { Suspended } \\
\text { Weight (g) }\end{array}$ & $\begin{array}{c}\text { Wet } \\
\text { Weight } \\
\text { (g) }\end{array}$ & $\begin{array}{c}\text { Green } \\
\text { Puck } \\
\text { Thick. } \\
\text { (in.) } \\
\end{array}$ & \begin{tabular}{|c|} 
Sintered \\
Puck \\
Thick. \\
(in.) \\
\end{tabular} & $\begin{array}{c}\text { Green } \\
\text { Density } \\
\text { (g/cc) }\end{array}$ & $\begin{array}{c}\text { Archi- } \\
\text { medes } \\
\text { Density } \\
(\mathrm{g} / \mathrm{cc}) \\
\end{array}$ & $\begin{array}{l}\text { Archi- } \\
\text { medes } \\
\text { Density } \\
(\% \mathrm{TD}) \\
\end{array}$ & $\begin{array}{c}\text { Geom. } \\
\text { Density } \\
\text { (g/cc) }\end{array}$ & $\begin{array}{l}\text { Geom. } \\
\text { (\% TD) }\end{array}$ & $\begin{array}{c}\text { Mass \% } \\
\text { Shrink- } \\
\text { age }\end{array}$ & $\begin{array}{c}\text { Volume } \\
\% \\
\text { Shinkage }\end{array}$ & \begin{tabular}{|c|} 
Diam. \\
$\%$ \\
Shrink- \\
age \\
\end{tabular} & $\begin{array}{l}\text { Powder } \\
\% \text { Moist }\end{array}$ \\
\hline \begin{tabular}{|l|} 
GA-31-12\%, BP \\
$6 / 19.6 .1$ tons
\end{tabular} & 2631 & 3.504 & 2.756 & 515.3 & 443.1 & 353.780 & 443.2 & 1.174 & 0.922 & 2.78 & 4.96 & 95.29 & 4.91 & 94.51 & 14.01 & 51.41 & 21.35 & 10.2 \\
\hline 6.5 tons & 2632 & 3.505 & 2.779 & 541.9 & 461.9 & 368.177 & 461.8 & 1.182 & 0.942 & 2.90 & 4.93 & 94.88 & 4.93 & 94.84 & 14.76 & 49.89 & 20.71 & 10.2 \\
\hline 6.3 tons & 2633 & 3.506 & 2.776 & 541.9 & 460.3 & 367.214 & 460.5 & 1.175 & 0.938 & 2.91 & 4.93 & 94.89 & 4.95 & 95.12 & 15.06 & 49.94 & 20.82 & 10.2 \\
\hline 4.2 tons & 2634 & 3.503 & 2.792 & 510.4 & 434.5 & 344.227 & 436.9 & 1.173 & 0.931 & 2.75 & 4.69 & 90.16 & 4.65 & 89.43 & 14.87 & 49.57 & 20.30 & 10.2 \\
\hline 4 tons & 2635 & 3.504 & 2.755 & 510.8 & 433.5 & 343.617 & 433.8 & 1.168 & 0.925 & 2.77 & 4.81 & 92.44 & 4.80 & 92.23 & 15.13 & 51.03 & 21.38 & 10.2 \\
\hline 1 tons & 2636 & 3.505 & 2.694 & 367.5 & 312.9 & 247.295 & 321.5 & 1.060 & 0.811 & 2.19 & 4.22 & 81.09 & 4.13 & 79.41 & 14.86 & 54.79 & 23.14 & 10.2 \\
\hline GA-31-14\% & 1704 & 3.257 & 2.594 & 500.1 & 420.3 & N/A & N/A & 1.233 & N/A & 2.97 & N/A & N/A & N/A & N/A & 15.96 & N/A & 20.36 & 11.5 \\
\hline GA-32-4\% CP & 1656 & 3.255 & 2.575 & 499.6 & 460.5 & 366.170 & 461.0 & 1.399 & 1.110 & 2.62 & 4.86 & 93.39 & 4.86 & 93.46 & 7.83 & 50.34 & 20.89 & 3.2 \\
\hline $\begin{array}{l}\text { GA-32-4\%, BP } \\
6 / 14, \text { G0207-2, } \\
4.9 \text { ton }\end{array}$ & 2586 & 3.508 & 2.726 & 473.7 & 422.0 & 336.816 & 422.1 & 1.145 & 0.900 & 2.61 & 4.95 & 95.16 & 4.90 & 94.25 & 10.91 & 52.53 & 22.29 & 3.2 \\
\hline 5.4 ton & 2587 & 3.505 & 2.728 & 459.9 & 422.9 & 337.397 & 423.0 & 1.148 & 0.900 & 2.53 & 4.94 & 95.00 & 4.90 & 94.31 & 8.05 & 52.50 & 22.17 & 3.2 \\
\hline 5.2 ton & 2588 & 3.505 & 2.722 & 457.9 & 421.2 & 336.258 & 421.2 & 1.148 & 0.893 & 2.52 & 4.96 & 95.36 & 4.94 & 95.09 & 8.01 & 53.08 & 22.34 & 3.2 \\
\hline 10 ton & 2589 & 3.510 & 2.770 & 494.7 & 455.2 & 363.870 & 455.3 & 1.174 & 0.931 & 2.66 & 4.98 & 95.74 & 4.95 & 95.18 & 7.98 & 50.60 & 21.08 & 3.2 \\
\hline 10.4 ton & 2590 & 3.510 & 2.766 & 494.7 & 455.3 & 364.147 & 455.3 & 1.174 & 0.932 & 2.66 & 4.99 & 96.06 & 4.96 & 95.38 & 7.96 & 50.69 & 21.20 & 3.2 \\
\hline GA-32-8\% CP & 1657 & 3.254 & 2.625 & 500.0 & 439.4 & 348.421 & 440.0 & 1.310 & 1.052 & 2.80 & 4.80 & 92.27 & 4.71 & 90.54 & 12.12 & 47.73 & 19.33 & 7.1 \\
\hline $\begin{array}{l}\text { GA-32-8\%, BP } \\
7 / 10,10.3 \text { tons }\end{array}$ & 2789 & 3.507 & 2.783 & 516.2 & 455.0 & 363.484 & 455.1 & 1.165 & 0.921 & 2.80 & 4.97 & 95.51 & 4.95 & 95.28 & 11.86 & 50.21 & 20.64 & 7.1 \\
\hline 17.5 tons & 2790 & 3.510 & 2.834 & 554.0 & 486.4 & 389.204 & 486.7 & 1.180 & 0.955 & 2.96 & 4.99 & 95.94 & 4.93 & 94.72 & 12.20 & 47.23 & 19.26 & 7.1 \\
\hline 9.5 tons & 2791 & 3.506 & 2.785 & 513.4 & 451.3 & 916 & 451.6 & 1.152 & 0.921 & 2.82 & 4.92 & 94.66 & 4 & 94.37 & 12.10 & 49.54 & 20.56 & 7.1 \\
\hline 5.1 tons & 2792 & 3.506 & 2.740 & 482.9 & 424.2 & 338.122 & 424.4 & 1.140 & 0.898 & 2.68 & 4.92 & 94.55 & & 93.99 & 12.16 & 51.88 & 21.85 & 7.1 \\
\hline \begin{tabular}{|l|}
4.1 tons \\
\end{tabular} & 2793 & 3.506 & 2.726 & 46 & 409.3 & 32 & 409.4 & 1.138 & 0.886 & 2.59 & 4.85 & 93.31 & 4.83 & 92.86 & 12.13 & 52.92 & 22.25 & 7.1 \\
\hline $\begin{array}{l}\text { GA-32-10\% Full } \\
\text { CP G0223-3 }\end{array}$ & 1825 & 3.254 & 2.587 & 501.8 & 431.8 & 344.436 & 431.9 & 1.273 & 1.013 & 2.89 & 4.94 & 94.94 & 4.95 & 95.14 & 13.95 & 49.69 & 20.50 & 8.9 \\
\hline $\begin{array}{l}\text { GA-32-10\%, } \\
\text { G0223-3, BP } \\
7 / 10,6.63 \text { tons }\end{array}$ & 2794 & 3.508 & 2.762 & 512.8 & 440.6 & 351.333 & 440.7 & 1.148 & 0.914 & 2.82 & 4.93 & 94.81 & 4.91 & 94.39 & 14.08 & 50.64 & 21.27 & 8.9 \\
\hline 6.9 tons & 2795 & 3.507 & 2.764 & 523.5 & 447.7 & 351.825 & 447.7 & 1.157 & 0.920 & 2.86 & 4.67 & 89.80 & 4.95 & 95.15 & 14.48 & 50.60 & 21.19 & 8.9 \\
\hline 6.1 tons & 2796 & 3.504 & 2.750 & 513.1 & 438.7 & 350.473 & 438.7 & 1.145 & 0.909 & 2.84 & 4.97 & 95.62 & 4.96 & 95.33 & 14.50 & 51.09 & 21.52 & 8.9 \\
\hline & 2797 & 3.506 & 2.746 & 507.8 & 434.6 & 347.008 & 434.6 & 1.151 & 0.906 & 2.79 & 4.96 & 95.42 & 4.94 & 95.02 & 14.42 & 51.70 & 21.68 & 8.9 \\
\hline 6.3 tons & 2798 & 3.507 & 2.752 & 513.3 & 439.0 & 350.466 & 439.1 & 1.149 & 0.911 & 2.82 & 4.95 & 95.25 & 4.94 & 95.04 & 14.47 & 51.17 & 21.53 & 8.9 \\
\hline 6.5 tons & 2799 & 3.505 & 2.758 & 517.4 & 442.6 & 353.436 & 442.7 & 1.156 & 0.915 & 2.83 & 4.96 & 95.35 & 4.94 & 94.99 & 14.46 & 50.98 & 21.31 & 8.9 \\
\hline ? Tons & 2800 & 3.504 & 2.734 & 501.6 & 428.7 & 342.300 & 428.9 & 1.142 & 0.901 & 2.78 & 4.95 & 95.20 & 4.94 & 95.08 & 14.53 & 51.96 & 21.97 & 8.9 \\
\hline 4.9 tons & 2801 & 3.506 & 2.727 & 497.0 & 425.3 & 339.489 & 425.4 & 1.146 & 0.899 & 2.74 & 4.95 & 95.20 & 4.94 & 95.02 & 14.43 & 52.53 & 22.22 & 8.9 \\
\hline \begin{tabular}{|l}
4 tons \\
\end{tabular} & 2802 & 3.503 & 2.718 & 484.8 & 414.2 & & 414.4 & 1.138 & 0.897 & 2.70 & 4.91 & 94.46 & 4.86 & 93.37 & 14.56 & 52.54 & 22.41 & 8.9 \\
\hline ? Tons & 2803 & 3.504 & 2.633 & 401.0 & 342.3 & 270.622 & 344.8 & 1.139 & 0.849 & 2.23 & 4.61 & 88.74 & 4.52 & 86.87 & 14.64 & 57.90 & 24.86 & 8.9 \\
\hline GA-32-12\% CP & 1658 & 3.254 & 2.630 & 499.9 & 430.0 & 341.299 & 431.4 & 1.264 & 1.025 & 2.90 & 4.77 & 91.78 & 4.71 & 90.60 & 13.98 & 47.02 & 19.18 & 8.7 \\
\hline
\end{tabular}

Page 30 of 49 
Appendix F - Puck Data for Granulation Matrix Testing

\begin{tabular}{|c|c|c|c|c|c|c|c|c|c|c|c|c|c|c|c|c|c|c|}
\hline Batch ID & $\begin{array}{c}\text { Puck } \\
\text { ID }\end{array}$ & $\begin{array}{c}\text { Green } \\
\text { Puck } \\
\text { Diam. } \\
\text { (in.) } \\
\end{array}$ & $\begin{array}{c}\text { Sintered } \\
\text { Puck } \\
\text { Diameter } \\
\text { (in.) } \\
\end{array}$ & $\begin{array}{c}\text { Green } \\
\text { Weight } \\
\text { (g) }\end{array}$ & $\begin{array}{c}\text { Sintered } \\
\text { Puck } \\
\text { Weight } \\
(\mathrm{g}) \\
\end{array}$ & $\begin{array}{c}\text { Puck } \\
\text { Suspended } \\
\text { Weight (g) }\end{array}$ & $\begin{array}{c}\text { Wet } \\
\text { Weight } \\
\text { (g) }\end{array}$ & $\begin{array}{c}\text { Green } \\
\text { Puck } \\
\text { Thick. } \\
\text { (in.) } \\
\end{array}$ & \begin{tabular}{|c|} 
Sintered \\
Puck \\
Thick. \\
(in.) \\
\end{tabular} & $\begin{array}{c}\text { Green } \\
\text { Density } \\
\text { (g/cc) }\end{array}$ & \begin{tabular}{|c|} 
Archi- \\
medes \\
Density \\
$(\mathrm{g} / \mathrm{cc})$ \\
\end{tabular} & $\begin{array}{c}\text { Archi- } \\
\text { medes } \\
\text { Density } \\
(\% \mathrm{TD}) \\
\end{array}$ & $\begin{array}{c}\text { Geom. } \\
\text { Density } \\
\text { (g/cc) }\end{array}$ & $\begin{array}{l}\text { Geom. } \\
\text { (\%TD) }\end{array}$ & $\begin{array}{c}\text { Mass \% } \\
\text { Shrink- } \\
\text { age }\end{array}$ & $\begin{array}{c}\text { Volume } \\
\% \\
\text { Shinkage }\end{array}$ & \begin{tabular}{|c|} 
Diam. \\
$\%$ \\
Shrink- \\
age \\
\end{tabular} & $\begin{array}{l}\text { Powder } \\
\text { \% Mois }\end{array}$ \\
\hline $\begin{array}{l}\mathrm{GA}-32-12 \%, \mathrm{BP} \\
6 \text { ton } \\
\end{array}$ & 2580 & 3.507 & 2.800 & 551.7 & 461.9 & 368.080 & 462.3 & 1.165 & 0.936 & 2.99 & 4.90 & 94.28 & 4.89 & 94.02 & 16.28 & 48.78 & 20.16 & 8.9 \\
\hline 5.3 ton & 2581 & 3.505 & 2.760 & 517.2 & 441.0 & 351.326 & 441.3 & 1.160 & 0.923 & 2.82 & 4.90 & 94.26 & 4.87 & 93.69 & 14.73 & 50.65 & 21.26 & 8.9 \\
\hline 4.3 ton & 2582 & 3.504 & 2.737 & 491.0 & 422.2 & 334.908 & 422.3 & 1.160 & 0.912 & 2.68 & 4.83 & 92.91 & 4.80 & 92.31 & 14.01 & 52.02 & 21.89 & 8.9 \\
\hline 3.8 ton & 2583 & 3.505 & 2.730 & 493.8 & 423.8 & 337.541 & 424.1 & 1.155 & 0.911 & 2.70 & 4.90 & 94.16 & 4.85 & 93.24 & 14.18 & 52.14 & 22.11 & 8.9 \\
\hline 4.5 ton & 2584 & 3.507 & 2.743 & 495.8 & 425.0 & 337.993 & 425.3 & 1.148 & 0.906 & 2.73 & 4.87 & 93.61 & 4.84 & 93.13 & 14.28 & 51.71 & 21.79 & 8.9 \\
\hline 4 ton & 2585 & 3.505 & 2.737 & 492.6 & 422.4 & 336.143 & 422.5 & 1.147 & 0.905 & 2.72 & 4.89 & 94.06 & 4.84 & 93.07 & 14.25 & 51.88 & 21.91 & 8.9 \\
\hline $\begin{array}{l}\text { GA-32-14\% CP } \\
\text { G0207-1 }\end{array}$ & 1661 & 3.254 & 2.636 & 500.0 & 418.5 & 332.878 & 418.5 & 1.192 & 0.965 & 3.08 & 4.89 & 94.00 & 4.85 & 93.23 & 16.30 & 46.86 & 18.99 & 11.5 \\
\hline $\begin{array}{l}\text { GA-32-14\%, BP } \\
6 / 14 . \text { G0407.2 }\end{array}$ & 2571 & N/A & & & & & & & & N/A & N/A & N/A & N/A & N/A & N/A & N/A & N/A & 10.3 \\
\hline 2.7 ton & 2572 & 3.506 & 2.711 & 478.0 & 401.3 & 318.465 & 402.3 & 1.155 & 0.905 & 2.62 & 4.79 & 92.05 & 4.69 & 90.12 & 16.05 & 53.14 & 22.68 & 10.3 \\
\hline 3.1 ton & 2573 & 3.506 & 2.712 & 491.1 & 410.7 & 326.251 & 411.7 & 1.150 & 0.903 & 1.05 & 4.81 & 92.43 & 4.80 & 92.37 & 16.37 & 53.01 & 22.65 & 10.3 \\
\hline 6 ton & 2574 & 3.509 & 2.799 & 503.2 & 421.1 & 336.132 & 421.3 & 1.073 & 0.856 & 2.96 & 4.94 & 95.08 & 4.88 & 93.79 & 16.32 & 49.23 & 20.23 & 10.3 \\
\hline 22.6 ton & 2575 & 3.511 & 2.837 & 550.7 & 466.7 & 373.613 & 467.2 & 1.128 & 0.917 & 3.08 & 4.99 & 95.90 & 4.91 & 94.45 & 15.25 & 46.91 & 19.20 & 10.3 \\
\hline \begin{tabular}{|l|} 
GA-32-16\% \\
\end{tabular} & 2088 & 3.253 & 2.608 & 501.2 & 414.2 & 329.945 & 415.3 & 1.229 & 0.991 & 2.99 & 4.85 & 93.32 & 4.77 & 91.79 & 17.36 & 48.16 & 19.83 & 12.0 \\
\hline GA-33-8\% & 1659 & 3.255 & 2.605 & 499.8 & 431.6 & 344.154 & 432.0 & 1.265 & 1.014 & 2.90 & 4.91 & 94.48 & 4.87 & 93.69 & 13.65 & 48.65 & 19.97 & 8.7 \\
\hline $\begin{array}{l}\text { GA-33-8\% } \\
\text { Repeat }\end{array}$ & 1708 & 3.254 & 2.616 & 499.0 & 432.1 & 344.430 & 432.7 & 1.252 & 1.007 & 2.92 & 4.90 & 94.14 & 4.87 & 93.66 & 13.41 & 48.01 & 19.61 & 7.5 \\
\hline GA-33-10\% & 1709 & 3.254 & 2.625 & 499.1 & 425.7 & 339.566 & 426.4 & 1.221 & 0.981 & 3.00 & 4.90 & 94.28 & 4.89 & 94.07 & 14.71 & 47.71 & 19.33 & 9.5 \\
\hline GA-33-12\% & 1660 & 3.251 & 2.607 & 500.0 & 415.6 & 330.565 & 416.9 & 1.249 & 1.003 & 2.94 & 4.81 & 92.57 & 4.74 & 91.07 & 16.88 & 48.35 & 19.81 & 12.2 \\
\hline \begin{tabular}{|l|} 
GA-34-8\% \\
\end{tabular} & 1751 & 3.254 & 2.592 & 498.5 & 440.7 & 350.203 & 440.7 & 1.325 & 1.050 & 2.76 & 4.87 & 93.65 & 4.85 & 93.32 & 11.59 & 49.71 & 20.34 & 6.9 \\
\hline \begin{tabular}{|l|} 
GA-34-8\% \\
\end{tabular} & 1781 & 3.254 & 2.586 & 500.1 & 442.2 & 352.580 & 442.7 & 1.331 & 1.056 & 2.74 & 4.91 & 94.36 & 4.86 & 93.53 & 11.58 & 49.88 & 20.53 & 6.9 \\
\hline GA-34-12\% & 1752 & 3.254 & 2.596 & 498.9 & 427.4 & 340.428 & 427.4 & 1.271 & 1.010 & 2.88 & 4.91 & 94.50 & 4.88 & 93.79 & 14.33 & 49.41 & 20.22 & 8.7 \\
\hline GA-34-12\% & 1780 & 3.254 & 2.591 & 497.5 & 427.9 & 341.064 & 428.1 & 1.274 & 1.018 & 2.87 & 4.92 & 94.55 & 4.86 & 93.53 & 13.99 & 49.33 & 20.37 & 8.7 \\
\hline GA-35-8\% & 1753 & 3.254 & 2.621 & 499.9 & 436.3 & 346.604 & 436.3 & 1.268 & 1.021 & 2.8 & 4.86 & 93.54 & 4.83 & 92.92 & 12.72 & 47.75 & 19.45 & 6.9 \\
\hline \begin{tabular}{|l|} 
GA-35-8\% \\
\end{tabular} & 1778 & 3.255 & 2.616 & 500.3 & 436.6 & 347.211 & 437.2 & 1.271 & 1.031 & 2.89 & 4.85 & 93.30 & 4.81 & 92.43 & 12.73 & 47.60 & 19.63 & 6.9 \\
\hline \begin{tabular}{|l} 
GA-35-12\% \\
\end{tabular} & 1754 & 3.253 & 2.635 & 499.3 & 425.6 & 337.950 & 425.6 & 1.228 & 0.990 & 2.99 & 4.86 & 93.38 & 4.81 & 92.49 & 14.76 & 47.09 & 19.00 & 8.1 \\
\hline \begin{tabular}{|l} 
GA-35-12\% \\
\end{tabular} & 1777 & 3.254 & 2.620 & 501.4 & 428.3 & 340.608 & 428.4 & 1.244 & 1.000 & 2.96 & 4.88 & 93.82 & 4.85 & 93.20 & 14.58 & 47.88 & 19.48 & 8.1 \\
\hline GA-36-8\% CP & 1774 & 3.255 & 2.604 & 500.1 & 439.2 & 349.847 & 439.6 & 1.298 & 1.041 & 2.83 & 4.89 & 94.10 & 4.83 & 92.94 & 12.18 & 48.66 & 20.00 & 6.6 \\
\hline $\begin{array}{l}\text { GA-36-8\%, BP } \\
6 / 28.4 \text { tons }\end{array}$ & 2637 & 3.506 & 2.720 & 463.4 & 407.8 & 322.801 & 408.9 & 1.160 & 0.909 & 2.52 & 4.74 & 91.08 & 4.71 & 90.58 & 12.00 & 52.83 & 22.42 & 8.0 \\
\hline \begin{tabular}{|l|l|l}
6.1 tons \\
\end{tabular} & 2638 & 3.505 & 2.745 & 490.0 & 430.5 & 341.763 & 431.4 & 1.169 & 0.929 & 2.6 & 4.80 & 92.36 & 4.78 & 91.86 & 12.14 & 51.25 & 21.68 & 8.0 \\
\hline 5.8 tons & 2639 & 3.505 & 2.765 & 498.6 & 437.5 & 347.482 & 438.5 & 1.174 & 0.925 & 2.69 & 4.81 & 92.44 & 4.81 & 92.41 & 12.25 & 50.96 & 21.11 & 8.0 \\
\hline 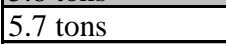 & 2640 & 3.506 & 2.755 & 497.7 & 436.6 & 347.760 & 437.6 & 1.175 & 0.928 & 2.68 & 4.86 & 93.46 & 4.81 & 92.59 & 12.28 & 51.22 & 21.42 & 8.0 \\
\hline 5.5 tons & 2641 & 3.506 & 2.750 & 493.7 & 433.5 & 344.501 & 434.3 & 1.169 & 0.912 & 2.67 & 4.83 & 92.84 & 4.88 & 93.89 & 12.19 & 51.99 & 21.56 & 8.0 \\
\hline \begin{tabular}{|l|} 
GA-36-12\% CP \\
\end{tabular} & 1779 & 3.255 & 2.610 & 499.9 & 425.8 & 339.112 & 425.7 & 1.235 & 0.990 & 2.97 & 4.92 & 94.57 & 4.90 & 94.31 & 14.82 & 48.45 & 19.82 & 9.3 \\
\hline $\begin{array}{l}\mathrm{GA}-36-12 \%, \mathrm{BP} \\
6 / 29.4 .5 \text { tons }\end{array}$ & 2657 & 3.506 & 2.762 & 527.1 & 440.5 & 351.030 & 440.7 & 1.163 & 0.920 & 2.86 & 4.91 & 94.47 & 4.88 & 93.75 & 16.43 & 50.90 & 21.22 & 9.3 \\
\hline $\begin{array}{l}10.1 \text { tons } \\
\end{array}$ & 2658 & 3.508 & 2.826 & 569 & 1.1 & 384.0 & 4 & 1.185 & 0.955 & 3 & 4.94 & 95.07 & 4.90 & 94.22 & 15.48 & 47.69 & 19.44 & 9.3 \\
\hline 6.2 tons & 2659 & 3.508 & 2.811 & 558.2 & 473.3 & 376.986 & 473.5 & 1.180 & 0.955 & 2.99 & 4.90 & 94.31 & 4.87 & 93.69 & 15.21 & 48.02 & 19.87 & 9.3 \\
\hline 6.25 tons & 2660 & 3.506 & 2.795 & 539.2 & 457.5 & 363.790 & 457.6 & 1.172 & 0.940 & 2.91 & 4.88 & 93.79 & 4.84 & 93.06 & 15.15 & 49.02 & 20.28 & 9.3 \\
\hline 5 tons & 2661 & 3.505 & 2.780 & 515.8 & 438.0 & 346.869 & 439.1 & 1.166 & 0.933 & 2.80 & 4.75 & 91.33 & 4.72 & 90.73 & 15.08 & 49.65 & 20.68 & 9.3 \\
\hline GA-37-6\% & 1830 & 3.256 & 2.627 & 498.6 & 446.0 & 353.851 & 450.1 & 1.332 & 1.091 & 2.74 & 4.63 & 89.11 & 4.60 & 88.48 & 10.55 & 46.67 & 19.32 & 4.8 \\
\hline GA-37-8\% & 1768 & 3.252 & 2.601 & 499.8 & 426.0 & 337.454 & 426.5 & 1.280 & 1.030 & 2.87 & 4.78 & 92.00 & 4.75 & 91.32 & 14.77 & 48.51 & 20.02 & 7.6 \\
\hline
\end{tabular}

Page 31 of 49 
Appendix F - Puck Data for Granulation Matrix Testing

\begin{tabular}{|c|c|c|c|c|c|c|c|c|c|c|c|c|c|c|c|c|c|c|}
\hline Batch ID & $\begin{array}{c}\text { Puck } \\
\text { ID }\end{array}$ & $\begin{array}{c}\text { Green } \\
\text { Puck } \\
\text { Diam. } \\
\text { (in.) }\end{array}$ & $\begin{array}{l}\text { Sintered } \\
\text { Puck } \\
\text { Diameter } \\
\text { (in.) }\end{array}$ & $\begin{array}{c}\text { Green } \\
\text { Weight } \\
\text { (g) }\end{array}$ & \begin{tabular}{c|} 
Sintered \\
Puck \\
Weight \\
$($ g)
\end{tabular} & $\begin{array}{c}\text { Puck } \\
\text { Suspended } \\
\text { Weight (g) }\end{array}$ & $\begin{array}{c}\text { Wet } \\
\text { Weight } \\
\text { (g) }\end{array}$ & $\begin{array}{c}\text { Green } \\
\text { Puck } \\
\text { Thick. } \\
\text { (in.) }\end{array}$ & $\begin{array}{c}\text { Sintered } \\
\text { Puck } \\
\text { Thick. } \\
\text { (in.) }\end{array}$ & $\begin{array}{c}\text { Green } \\
\text { Density } \\
\text { (g/cc) }\end{array}$ & $\begin{array}{c}\text { Archi- } \\
\text { medes } \\
\text { Density } \\
(\mathrm{g} / \mathrm{cc})\end{array}$ & $\begin{array}{c}\text { Archi- } \\
\text { medes } \\
\text { Density } \\
(\% \mathrm{TD})\end{array}$ & $\begin{array}{c}\text { Geom. } \\
\text { Density } \\
(\mathrm{g} / \mathrm{cc})\end{array}$ & $\begin{array}{l}\text { Geom. } \\
\text { (\% TD) }\end{array}$ & $\begin{array}{c}\text { Mass \% } \\
\text { Shrink- } \\
\text { age }\end{array}$ & $\begin{array}{c}\text { Volume } \\
\% \\
\text { Shinkage }\end{array}$ & $\begin{array}{c}\text { Diam. } \\
\% \\
\text { Shrink- } \\
\text { age }\end{array}$ & $\begin{array}{l}\text { Powder } \\
\% \text { Moist }\end{array}$ \\
\hline GA-37-10\% & 1831 & 3.254 & 2.662 & $\begin{array}{ll}498.8 \\
\end{array}$ & 424.5 & 336.781 & 428.7 & 1.225 & 1.010 & 2.99 & 4.62 & 88.81 & 4.61 & 88.60 & 14.90 & 44.81 & 18.19 & 9.2 \\
\hline GA-37-12\% & 1769 & 3.253 & 2.628 & $\begin{array}{ll}498.9 \\
\end{array}$ & 416.1 & 329.906 & 418.3 & 1.243 & 1.008 & 2.95 & 4.71 & 90.53 & 4.64 & 89.28 & 16.60 & 47.06 & 19.21 & 9.6 \\
\hline GA-38-8\% & 1770 & 3.255 & 2.539 & 500.9 & 434.9 & 346.649 & 434.9 & 1.372 & 1.074 & 2.68 & 4.93 & 94.77 & 4.88 & 93.83 & 13.18 & 52.36 & 22.00 & 7.7 \\
\hline GA-38-12\% & 1773 & 3.254 & 2.555 & 500.2 & 419.1 & 334.422 & 419.4 & 1.305 & 1.024 & 2.81 & 4.93 & 94.84 & 4.87 & 93.65 & 16.21 & 51.61 & 21.48 & 10.9 \\
\hline \begin{tabular}{|l|} 
GA-39-10\% CP \\
\end{tabular} & 1833 & 3.254 & 2.586 & 498.3 & 429.5 & 342.495 & 429.6 & 1.278 & 1.018 & 2.86 & 4.93 & 94.82 & 4.90 & 94.24 & 13.81 & 49.68 & 20.53 & 8.3 \\
\hline $\begin{array}{l}\text { GA- } 39-10 \%, \text { BP } \\
6 / 28.3 .5 \text { tons }\end{array}$ & 2642 & 3.506 & 2.737 & 475.6 & 413.4 & 327.697 & 414.6 & 1.160 & 0.919 & 2.59 & 4.76 & 91.48 & 4.66 & 89.70 & 13.08 & 51.71 & 21.93 & 10.0 \\
\hline 2.8 tons & 2643 & 3.506 & 2.691 & 471.8 & 404.0 & 322.161 & 404.9 & 1.168 & 0.898 & 2.55 & 4.88 & 93.90 & 4.83 & 92.80 & 14.37 & 54.70 & 23.25 & 10.0 \\
\hline 4.7 tons & 2644 & 3.505 & 2.734 & 504.1 & 431.0 & 344.915 & 432.0 & 1.168 & 0.917 & 2.73 & 4.95 & 95.18 & 4.88 & 93.93 & 14.50 & 52.22 & 22.00 & 10.0 \\
\hline 4.6 tons & 2645 & 3.505 & 2.727 & 501.4 & 429.6 & 343.310 & 430.3 & 1.169 & 0.912 & 2.71 & 4.94 & 94.97 & 4.92 & 94.62 & 14.32 & 52.77 & 22.20 & 10.0 \\
\hline 4.4 tons & 2646 & 3.506 & 2.720 & 497.4 & 424.2 & 338.688 & 424.9 & 1.165 & 0.914 & 2.70 & 4.92 & 94.62 & 4.87 & 93.70 & 14.72 & 52.77 & 22.42 & 10.0 \\
\hline \begin{tabular}{|l|} 
GA-39-12\% CP \\
\end{tabular} & 1838 & 3.254 & 2.612 & 499.3 & 419.1 & 334.465 & 419.8 & 1.232 & 0.985 & 2.97 & 4.91 & 94.45 & 4.84 & 93.16 & 16.06 & 48.48 & 19.73 & 10.4 \\
\hline $\begin{array}{l}\mathrm{GA}-39-12 \%, \mathrm{BP} \\
6 / 28,6.3 \text { tons } \\
\end{array}$ & 2652 & 3.506 & 2.743 & 512.6 & 444.1 & 355.695 & 445.2 & 1.181 & 0.933 & 2.74 & 4.96 & 95.42 & 4.91 & 94.50 & 13.36 & 51.63 & 21.76 & 12.0 \\
\hline 3.8 tons & 2653 & 3.506 & 2.731 & 507.7 & 427.1 & 340.979 & 428.1 & 1.166 & 0.916 & 2.75 & 4.90 & 94.28 & 4.86 & 93.38 & 15.88 & 52.32 & 22.10 & 12.0 \\
\hline 3 tons & 2654 & 3.505 & 2.729 & 507.9 & 424.4 & 338.588 & 425.4 & 1.175 & 0.915 & 2.73 & 4.89 & 94.01 & 4.84 & 93.03 & 16.44 & 52.78 & 22.14 & 12.0 \\
\hline 3 tons & 2655 & 3.505 & 2.734 & 507.4 & 423.7 & 336.415 & 423.8 & 1.172 & 0.916 & 2.74 & 4.85 & 93.24 & 4.81 & 92.44 & 16.50 & 52.44 & 22.00 & 12.0 \\
\hline 2.7 tons & 2656 & 3.505 & 2.725 & 512.6 & 427.6 & 340.078 & 428.6 & 1.188 & 0.931 & 2.73 & 4.83 & 92.89 & 4.80 & 92.39 & 16.58 & 52.62 & 22.25 & 12.0 \\
\hline \begin{tabular}{|l|} 
GA-39-14\% CP \\
\end{tabular} & 1839 & 3.253 & 2.604 & 498.4 & 415.1 & 331.097 & 415.9 & 1.228 & 0.977 & 2.98 & 4.89 & 94.13 & 4.87 & 93.59 & 16.71 & 49.01 & 19.95 & 11.4 \\
\hline $\begin{array}{l}\mathrm{GA}-39-14 \%, \mathrm{BP} \\
6 / 29.2 .3 \text { tons }\end{array}$ & 2667 & 3.505 & 2.760 & 506.0 & 421.6 & 334.056 & 422.8 & 1.153 & 0.910 & 2.78 & 4.75 & 91.36 & 4.72 & 90.85 & 16.68 & 51.05 & 21.26 & 11.4 \\
\hline 2.2 tons & 2668 & 3.504 & 2.736 & 4997.1 & 411.5 & 326.753 & \begin{tabular}{ll|}
411.8 \\
\end{tabular} & 1.137 & 0.889 & 2.77 & 4.84 & 93.05 & 4.80 & 92.36 & 17.22 & 52.32 & 21.92 & 11.4 \\
\hline 2.3 tons & 2669 & 3.504 & 2.740 & 499.2 & 412.8 & 327.812 & 412.9 & 1.135 & 0.888 & 2.78 & 4.85 & 93.30 & 4.81 & 92.49 & 17.31 & 52.15 & 21.80 & 11.4 \\
\hline 5 tons & 2670 & 3.508 & 2.779 & 536.0 & 443.6 & 354.588 & 443.9 & 1.147 & 0.907 & 2.95 & 4.97 & 95.52 & 4.92 & 94.60 & 17.24 & 50.37 & 20.78 & 11.4 \\
\hline 3.6 ton & 2671 & 3.505 & 2.756 & 521.6 & 430.1 & 343.257 & 430.1 & 1.149 & 0.898 & 2.87 & 4.95 & 95.24 & 4.90 & 94.19 & 17.54 & 51.67 & 21.37 & 11.4 \\
\hline 2.5 ton & 2672 & 3.505 & 2.742 & 505.3 & 417.1 & 331.848 & 417.3 & 1.134 & 0.889 & 2.82 & 4.88 & 93.87 & 4.85 & 93.21 & 17.45 & 52.01 & 21.77 & 11.4 \\
\hline \begin{tabular}{|l|} 
GA- $40-10 \%$ CP \\
\end{tabular} & 1840 & 3.255 & 2.575 & 500.1 & 437.5 & 349.517 & \begin{tabular}{ll|}
437.8 \\
\end{tabular} & 1.319 & 1.045 & 2.78 & 4.96 & 95.30 & 4.90 & 94.31 & 12.52 & 50.41 & 20.89 & 7.4 \\
\hline $\begin{array}{l}\text { GA- } 40-10 \%, \mathrm{BP} \\
6 / 28.4 .5 \text { tons }\end{array}$ & 2647 & 3.507 & 2.724 & 496.4 & 426.8 & 341.145 & 427.9 & 1.167 & 0.904 & 2.69 & 4.92 & 94.61 & 4.94 & 95.04 & 14.02 & 53.26 & 22.33 & 10.0 \\
\hline 6 tons & 2648 & 3.505 & 2.727 & 503.6 & 437.1 & 350.212 & 438.2 & 1.177 & 0.920 & 2.71 & 4.97 & 95.53 & 4.96 & 95.43 & 13.20 & 52.68 & 22.20 & 10.0 \\
\hline & 2649 & 3.505 & 2.725 & 501.5 & 436.4 & 349.723 & 437.5 & 1.173 & 0.923 & 2.70 & 4.97 & 95.61 & 4.95 & 95.11 & 12.98 & 52.43 & 22.25 & 10.0 \\
\hline & 2650 & 3.506 & 2.726 & 500.2 & 435.9 & 349.163 & 436.3 & 1.171 & 0.925 & 2.70 & 5.00 & 96.20 & 4.93 & 94.73 & 12.85 & 52.24 & 22.25 & 10.0 \\
\hline 5.6 tons & 2651 & 3.506 & 2.725 & 496.8 & 432.6 & 346.368 & 434.0 & 1.181 & 0.908 & 2.66 & 4.94 & 94.93 & 4.98 & 95.84 & 12.92 & 53.55 & 22.28 & 10.0 \\
\hline \begin{tabular}{|l|} 
GA- $40-12 \%$ CP \\
\end{tabular} & 1841 & 3.253 & 2.628 & 500.0 & 419.3 & 333.911 & 420.1 & 1.210 & 0.979 & 3.03 & 4.86 & 93.56 & 4.82 & 92.63 & 16.14 & 47.18 & 19.21 & 10.0 \\
\hline $\begin{array}{l}\mathrm{GA}-40-12 \%, \mathrm{BP} \\
6 / 29.3 .7 \text { tons }\end{array}$ & 2662 & 3.506 & 2.775 & 501.3 & 424.3 & 335.840 & 427.2 & 1.163 & 0.930 & 2.72 & 4.64 & 89.31 & 4.60 & 88.50 & 15.36 & 49.89 & 20.85 & 10.0 \\
\hline 1.3 tons & 2663 & 3.502 & 2.729 & 440.5 & 368.7 & 291.418 & 374.9 & 1.125 & 0.886 & 2.48 & 4.42 & 84.93 & 4.34 & 83.46 & 16.30 & 52.17 & 22.07 & 10.0 \\
\hline 1.6 tons & 2664 & 3.504 & 2.742 & 458.7 & 383.2 & & 388.9 & 1.140 & 0.900 & 2.55 & 4.45 & 85.66 & 4.40 & 99 & 16.46 & 51.65 & 21.75 & 10.0 \\
\hline 2.3 tons & 2665 & 3.504 & 2.765 & 508.1 & 423.7 & 335.460 & 424.7 & 1.159 & 0.917 & 2.77 & 4.75 & 91.31 & 4.69 & 90.28 & 16.61 & 50.72 & 21.09 & 10.0 \\
\hline 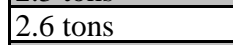 & 2666 & 3.505 & 2.763 & 510.6 & 425.4 & 337.297 & 425.8 & 1.146 & 0.910 & 2.82 & 4.81 & 92.43 & 4.76 & 91.47 & 16.69 & 50.65 & 21.17 & 10.0 \\
\hline GA-41-10\% & 1937 & 3.254 & & 500.2 & N/A & N/A & N/A & 1.285 & N/A & 2.86 & N/A & N/A & N/A & N/A & N/A & N/A & N/A & 7.9 \\
\hline \begin{tabular}{|l|} 
GA-41-14\% \\
G0309-1
\end{tabular} & 1938 & 3.255 & 2.558 & 500.3 & 431.9 & 344.058 & 431.9 & 1.328 & 1.046 & 2.76 & 4.92 & 94.55 & 4.90 & 94.26 & 13.67 & 51.35 & 21.41 & 8.6 \\
\hline $\begin{array}{l}\text { GA-41-14\%, } \\
\text { G0310-0 }\end{array}$ & 2774 & 3.255 & 2.570 & 499.6 & 421.2 & 336.529 & 422.1 & 1.277 & 1.023 & 2.87 & 4.92 & 94.66 & 4.84 & 93.11 & 15.69 & 50.05 & 21.04 & 8.6 \\
\hline
\end{tabular}

Page 32 of 49 
Appendix F - Puck Data for Granulation Matrix Testing

\begin{tabular}{|c|c|c|c|c|c|c|c|c|c|c|c|c|c|c|c|c|c|c|}
\hline Batch ID & $\begin{array}{c}\text { Puck } \\
\text { ID }\end{array}$ & $\begin{array}{c}\text { Green } \\
\text { Puck } \\
\text { Diam. } \\
\text { (in.) }\end{array}$ & $\begin{array}{c}\text { Sintered } \\
\text { Puck } \\
\text { Diameter } \\
\text { (in.) }\end{array}$ & $\begin{array}{c}\text { Green } \\
\text { Weight } \\
\text { (g) }\end{array}$ & \begin{tabular}{|c} 
Sintered \\
Puck \\
Weight \\
(g)
\end{tabular} & $\begin{array}{c}\text { Puck } \\
\text { Suspended } \\
\text { Weight (g) }\end{array}$ & $\begin{array}{c}\text { Wet } \\
\text { Weight } \\
\text { (g) }\end{array}$ & $\begin{array}{c}\text { Green } \\
\text { Puck } \\
\text { Thick. } \\
\text { (in.) }\end{array}$ & \begin{tabular}{|c|} 
Sintered \\
Puck \\
Thick. \\
(in.)
\end{tabular} & $\begin{array}{c}\text { Green } \\
\text { Density } \\
\text { (g/cc) }\end{array}$ & $\begin{array}{c}\text { Archi- } \\
\text { medes } \\
\text { Density } \\
\text { (g/cc) }\end{array}$ & $\begin{array}{c}\text { Archi- } \\
\text { medes } \\
\text { Density } \\
(\% \mathrm{TD})\end{array}$ & $\begin{array}{c}\text { Geom. } \\
\text { Density } \\
\text { (g/cc) }\end{array}$ & $\begin{array}{l}\text { Geom. } \\
\text { (\%TD) }\end{array}$ & $\begin{array}{c}\text { Mass \% } \\
\text { Shrink- } \\
\text { age }\end{array}$ & $\begin{array}{c}\text { Volume } \\
\% \\
\text { Shinkage }\end{array}$ & \begin{tabular}{|c|} 
Diam. \\
$\%$ \\
Shrink- \\
age
\end{tabular} & $\begin{array}{l}\text { Powder } \\
\% \text { Moist }\end{array}$ \\
\hline $\begin{array}{l}\text { GA-41-14\%, } \\
\text { Full G0310-1 }\end{array}$ & 2775 & 3.246 & 2.530 & 500.5 & 405.6 & 322.485 & 407.6 & 1.347 & 1.146 & 2.74 & 4.77 & 91.64 & 4.29 & 82.59 & 18.96 & 48.31 & 22.06 & 11.3 \\
\hline GA-42-14\% & 1842 & 3.253 & 2.599 & 499.6 & 415.3 & 331.329 & 416.3 & 1.237 & 0.987 & 2.97 & 4.89 & 93.99 & 4.84 & 93.05 & 16.87 & 49.06 & 20.10 & 11.3 \\
\hline GA-44-12\% & 1837 & 3.254 & 2.589 & 499.6 & 427.7 & 341.382 & 427.7 & 1.269 & 1.008 & 2.89 & 4.95 & 95.29 & 4.92 & 94.56 & 14.39 & 49.71 & 20.44 & 10.1 \\
\hline $\begin{array}{l}\text { GA-45-12\%, } \\
\text { G0425-2, BP } \\
7 / 13,4.4 \text { tons }\end{array}$ & 2811 & 3.507 & 2.670 & 486.3 & 405.7 & 320.840 & 405.9 & 1.231 & 0.929 & 2.50 & 4.77 & 91.72 & 4.76 & 91.50 & 16.57 & 56.25 & 23.87 & 8.2 \\
\hline \multirow[t]{2}{*}{5 tons } & 2812 & 3.506 & 2.671 & 499.2 & 417.1 & N/A & N/A & 1.262 & 0.960 & 2.50 & N/A & N/A & 4.73 & 90.97 & 16.45 & 55.84 & 23.82 & 8.2 \\
\hline & 2813 & 3.508 & 2.700 & 505.6 & 425.8 & 336.063 & 425.8 & 1.258 & 0.958 & 2.54 & 4.74 & 91.25 & 4.74 & 91.07 & 15.78 & 54.88 & 23.03 & 8.2 \\
\hline 5.2 tons & 2814 & 3.507 & 2.690 & 499.6 & 420.7 & 333.163 & 420.9 & 1.240 & 0.947 & 2.54 & 4.80 & 92.21 & 4.77 & 91.70 & 15.79 & 55.06 & 23.30 & 8.2 \\
\hline 5.4 tons & 2815 & 3.507 & 2.696 & 508.7 & 428.5 & 338.868 & 428.9 & 1.261 & 0.967 & 2.55 & 4.76 & 91.53 & 4.74 & 91.07 & 15.77 & 54.67 & 23.13 & 8.2 \\
\hline 5.3 tons & 2816 & 3.507 & 2.705 & 505.9 & 426.6 & 336.650 & 426.8 & 1.254 & 0.960 & 2.55 & 4.73 & 91.00 & 4.72 & 90.72 & 15.68 & 54.45 & 22.87 & 8.2 \\
\hline 6 tons & 2817 & 3.507 & 2.693 & 496.0 & 418.2 & 332.469 & 418.5 & 1.208 & 0.926 & 2.59 & 4.86 & 93.48 & 4.84 & 93.02 & 15.69 & 54.79 & 23.21 & 8.2 \\
\hline 5.9 tons & 2818 & 3.508 & 2.700 & 500.2 & 421.6 & 334.605 & 421.9 & 1.220 & 0.937 & 2.59 & 4.83 & 92.88 & 4.79 & 92.19 & 15.71 & 54.49 & 23.03 & 8.2 \\
\hline 5.2 tons & 2819 & 3.512 & 2.716 & 500.6 & 421.7 & 335.212 & 421.9 & 1.198 & 0.922 & 2.63 & 4.86 & 93.55 & 4.82 & 92.62 & 15.76 & 53.96 & 22.67 & 8.2 \\
\hline \multirow[t]{2}{*}{6.2 tons } & 2820 & 3.507 & 2.690 & 501.3 & 422.2 & 335.575 & 422.1 & 1.219 & 0.933 & 2.60 & 4.88 & 93.84 & 4.86 & 93.41 & 15.78 & 54.96 & 23.30 & 9.4 \\
\hline & 2821 & 3.508 & 2.700 & 502.7 & 423.1 & N/A & N/A & 1.221 & 0.940 & 2.60 & N/A & N/A & 4.80 & 92.23 & 15.83 & 54.39 & 23.03 & 9.4 \\
\hline 6 tons & 2822 & 3.508 & 2.687 & 496.3 & 417.6 & 332.157 & 417.4 & 1.206 & 0.921 & 2.60 & 4.90 & 94.21 & 4.88 & 93.81 & 15.86 & 55.19 & 23.40 & 9.4 \\
\hline 6.1 tons & 2823 & 3.507 & 2.690 & 495.5 & 417.2 & 331.320 & 416.8 & 1.205 & 0.922 & 2.60 & 4.88 & 93.86 & 4.86 & 93.41 & 15.80 & 54.97 & 23.30 & 9.4 \\
\hline 5.4 tons & 2824 & 3.507 & 2.714 & 448.4 & 377.6 & 301.211 & 377.9 & 1.045 & 0.815 & 2.71 & 4.92 & 94.69 & 4.89 & 93.96 & 15.79 & 53.28 & 22.61 & 9.4 \\
\hline 10.6 tons & 2825 & 3.507 & 2.740 & 427.0 & 352.1 & 281.052 & 352.7 & 0.980 & 0.754 & 2.75 & 4.91 & 94.51 & 4.83 & 92.91 & 17.54 & 53.03 & 21.87 & 9.4 \\
\hline $\begin{array}{l}\text { GA-45-12\%, } \\
\text { G0426, BP } 7 / 19, \\
12.8 \text { tons }\end{array}$ & 2826 & 3.508 & 2.754 & 511.8 & 431.9 & 344.251 & 432.4 & 1.164 & 0.913 & 2.78 & 4.90 & 94.22 & 4.84 & 93.17 & 15.61 & 51.65 & 21.49 & 9.4 \\
\hline 8.2 tons & 2827 & 3.503 & 2.798 & 525.3 & 446.0 & 355.192 & 446.5 & 1.152 & 0.915 & 2.89 & 4.88 & 93.93 & 4.84 & 93.00 & 15.10 & 49.32 & 20.13 & 9.4 \\
\hline 12 tons & 2828 & 3.505 & 2.828 & 557.6 & 475.0 & 379.288 & 475.4 & 1.165 & 0.942 & 3.03 & 4.94 & 95.04 & 4.90 & 94.18 & 14.81 & 47.35 & 19.32 & 9.4 \\
\hline 10 tons & 2829 & 3.505 & 2.812 & 544.0 & 463.1 & 369.625 & 463.4 & 1.155 & 0.930 & 2.98 & 4.94 & 94.97 & 4.89 & 94.07 & 14.87 & 48.16 & 19.77 & 9.4 \\
\hline \multirow[t]{2}{*}{\begin{tabular}{|l|}
5.3 tons \\
\end{tabular}} & 2830 & 3.503 & 2.795 & 516.5 & 439.8 & N/A & N/A & 1.145 & 0.917 & 2.86 & N/A & N/A & 4.77 & 91.71 & 14.85 & 49.01 & 20.21 & 9.4 \\
\hline & 2831 & 3.504 & 2.795 & 521.3 & 443.6 & 352.653 & 444.4 & 1.147 & 0.917 & 2.88 & 4.84 & 92.98 & 4.81 & 92.50 & 14.91 & 49.12 & 20.23 & 9.4 \\
\hline \multirow[t]{3}{*}{$\begin{array}{l}\text { GB-1-12\% } \\
\mathrm{Hf} / \mathrm{Ce} / \mathrm{U} \\
\end{array}$} & U300 & 3.500 & 2.748 & 509.7 & 457.3 & 370.880 & 458.2 & 1.141 & 0.895 & 2.83 & 5.24 & 91.71 & 5.26 & 92.04 & 10.28 & 51.64 & 21.49 & 5.4 \\
\hline & U301 & 3.500 & 2.739 & 499.9 & 449.5 & 364.940 & 450.6 & 1.120 & 0.878 & 2.83 & 5.25 & 91.89 & 5.30 & 92.82 & 10.09 & 51.98 & 21.74 & 5.4 \\
\hline & $\mathrm{U} 302$ & 3.500 & 2.742 & 499.8 & 447.2 & 363.290 & 447.3 & 1.112 & 0.880 & 2.85 & 5.32 & 93.23 & 5.25 & 91.94 & 10.52 & 51.42 & 21.66 & 5.4 \\
\hline \multirow[t]{2}{*}{ Sched 3} & U264 & 3.500 & 2.741 & 572.5 & 514.8 & 417.300 & 515.0 & 1.285 & 1.019 & 2.83 & 5.27 & 92.24 & 5.22 & 91.47 & 10.08 & 51.36 & 21.69 & 5.3 \\
\hline & $\mathrm{U} 265$ & 3.500 & 2.747 & 574.9 & 517.3 & 419.100 & 517.5 & 1.291 & 1.021 & 2.82 & 5.25 & 92.03 & 5.22 & 91.34 & 10.02 & 51.27 & 21.51 & 5.3 \\
\hline $15000 \mathrm{lbs}$ & $\mathrm{U} 266$ & 3.500 & 2.722 & 553.5 & 498.1 & 403.450 & 498.4 & 1.279 & 1.001 & 2.74 & 5.25 & 91.92 & 5.22 & 91.36 & 10.01 & 52.65 & 22.23 & 5.3 \\
\hline $15000 \mathrm{lbs}$ & U267 & 3.500 & & 396.8 & & & & 0.918 & 0.717 & 2.74 & N/A & N/A & N/A & N/A & N/A & N/A & N/A & 5.3 \\
\hline \multirow[t]{5}{*}{$\mathrm{Hf} / \mathrm{Ce} / \mathrm{U}$} & U237 & 3.500 & 2.745 & 500.1 & 433.3 & 351.520 & 433.4 & 1.076 & 0.845 & 2.95 & 5.29 & 92.66 & 5.29 & 92.57 & 13.36 & 51.69 & 21.57 & 8.4 \\
\hline & $\mathrm{U} 238$ & 3.500 & 2.733 & 498.6 & 432.2 & 350.530 & 432.3 & 1.085 & 0.848 & 2.91 & 5.29 & 92.56 & 5.30 & 92.83 & 13.31 & 52.34 & 21.91 & 8.4 \\
\hline & U239 & 3.500 & 2.725 & 500.1 & 433.3 & 351.380 & 433.3 & 1.092 & 0.852 & 2.90 & 5.29 & 92.65 & 5.32 & 93.16 & 13.36 & 52.70 & 22.14 & 8.4 \\
\hline & $\mathrm{U} 262$ & 3.500 & 2.757 & 565.5 & 492.7 & 397.830 & 493.1 & 1.234 & 0.978 & 2.91 & 5.17 & 90.56 & 5.15 & 90.16 & 12.87 & 50.81 & 21.23 & 7.9 \\
\hline & U263 & 3.500 & 2.737 & 583.1 & 509.1 & 413.090 & 509.1 & 1.279 & 0.997 & 2.89 & 5.30 & 92.86 & 5.29 & 92.73 & 12.69 & 52.32 & 21.80 & 7.9 \\
\hline
\end{tabular}

Page 33 of 49 
Appendix F - Puck Data for Granulation Matrix Testing

\begin{tabular}{|c|c|c|c|c|c|c|c|c|c|c|c|c|c|c|c|c|c|c|}
\hline Batch ID & $\begin{array}{c}\text { Puck } \\
\text { ID }\end{array}$ & $\begin{array}{c}\begin{array}{c}\text { Green } \\
\text { Puck } \\
\text { Diam. } \\
\text { (in.) }\end{array} \\
\end{array}$ & $\begin{array}{c}\text { Sintered } \\
\text { Puck } \\
\text { Diameter } \\
\text { (in.) } \\
\end{array}$ & $\begin{array}{c}\text { Green } \\
\text { Weight } \\
\text { (g) }\end{array}$ & $\begin{array}{c}\text { Sintered } \\
\text { Puck } \\
\text { Weight } \\
(\mathrm{g}) \\
\end{array}$ & $\begin{array}{c}\text { Puck } \\
\text { Suspended } \\
\text { Weight (g) }\end{array}$ & $\begin{array}{c}\text { Wet } \\
\text { Weight } \\
\text { (g) }\end{array}$ & $\begin{array}{c}\text { Green } \\
\text { Puck } \\
\text { Thick. } \\
\text { (in.) } \\
\end{array}$ & \begin{tabular}{|c} 
Sintered \\
Puck \\
Thick. \\
(in.) \\
\end{tabular} & $\begin{array}{c}\text { Green } \\
\text { Density } \\
\text { (g/cc) }\end{array}$ & \begin{tabular}{|c|} 
Archi- \\
medes \\
Density \\
$(\mathrm{g} / \mathrm{cc})$ \\
\end{tabular} & $\begin{array}{c}\text { Archi- } \\
\text { medes } \\
\text { Density } \\
(\% \mathrm{TD})\end{array}$ & $\begin{array}{c}\text { Geom. } \\
\text { Density } \\
\text { (g/cc) }\end{array}$ & $\begin{array}{l}\text { Geom. } \\
\text { (\%TD) }\end{array}$ & $\begin{array}{c}\text { Mass \% } \\
\text { Shrink- } \\
\text { age }\end{array}$ & $\begin{array}{c}\text { Volume } \\
\% \\
\text { Shinkage }\end{array}$ & \begin{tabular}{|c|} 
Diam. \\
$\%$ \\
Shrink- \\
age \\
\end{tabular} & $\begin{array}{l}\text { Powder } \\
\text { \% Mois }\end{array}$ \\
\hline \multirow{3}{*}{$\begin{array}{l}\mathrm{GB}-2-12 \% \\
\mathrm{Hf} / \mathrm{Ce} / \mathrm{U} \\
\end{array}$} & U222 & 3.501 & 2.744 & 499.7 & 445.1 & 361.150 & 445.1 & 1.111 & 0.880 & 2.85 & 5.30 & 92.83 & 5.22 & 91.38 & 10.92 & 51.33 & 21.62 & 6.0 \\
\hline & \begin{tabular}{|l|}
$\mathrm{U} 223$ \\
\end{tabular} & 3.501 & 2.748 & 500.6 & 446.5 & 362.060 & \begin{tabular}{|l|}
446.5 \\
\end{tabular} & 1.120 & 0.890 & 2.83 & 5.29 & 92.63 & 5.16 & 90.36 & 10.82 & 51.03 & 21.51 & 6.0 \\
\hline & $\mathrm{U} 224$ & 3.501 & 2.745 & 498.8 & 445.3 & 360.800 & \begin{tabular}{|l|}
445.3 \\
\end{tabular} & 1.122 & 0.893 & 2.82 & 5.27 & 92.26 & 5.14 & 90.01 & 10.74 & 51.06 & 21.59 & 6.0 \\
\hline \multirow[t]{3}{*}{ Sched 3} & $\mathrm{U} 231$ & 3.500 & 2.738 & 559.6 & 499.3 & 405.010 & 499.6 & 1.246 & 0.989 & 2.85 & 5.28 & 92.44 & 5.23 & 91.61 & 10.78 & 51.42 & 21.77 & 6.0 \\
\hline & $\mathrm{U} 232$ & 3.500 & 2.738 & 558.4 & 499.2 & 404.720 & \begin{tabular}{|l|}
499.5 \\
\end{tabular} & 1.250 & 0.984 & 2.83 & 5.27 & 92.22 & 5.26 & 92.06 & 10.60 & 51.82 & 21.77 & 6.0 \\
\hline & $\mathrm{U} 233$ & 3.500 & 2.740 & 557.5 & 498.3 & 404.000 & 498.6 & 1.246 & 0.971 & 2.84 & 5.27 & 92.29 & 5.31 & 92.98 & 10.62 & 52.23 & 21.71 & 6.0 \\
\hline \multirow[t]{6}{*}{$\begin{array}{l}\mathrm{GB}-3-10 \% \\
\mathrm{Hf} / \mathrm{Ce} / \mathrm{U} \\
\end{array}$} & U225 & 3.500 & 2.800 & 499.7 & 430.5 & 349.750 & 430.5 & 1.003 & 0.798 & 3.16 & 5.33 & 93.41 & 5.34 & 93.59 & 13.86 & 49.07 & 20.00 & 10.2 \\
\hline & U226 & 3.500 & 2.800 & 501.5 & 431.7 & 350.810 & \begin{tabular}{|l|}
431.7 \\
\end{tabular} & 1.008 & 0.814 & 3.16 & 5.34 & 93.47 & 5.25 & 92.02 & 13.92 & 48.31 & 20.00 & 10.2 \\
\hline & $\begin{array}{l}\mathrm{U} 227 \\
\end{array}$ & 3.500 & 2.789 & 500.7 & 432.3 & 351.280 & \begin{tabular}{|l|}
432.3 \\
\end{tabular} & 1.015 & 0.810 & 3.13 & 5.33 & 93.43 & 5.33 & 93.34 & 13.66 & 49.32 & 20.31 & 10.2 \\
\hline & U248 & 3.501 & 2.800 & 581.0 & 502.4 & 408.120 & 502.7 & 1.182 & 0.952 & 3.12 & 5.31 & 93.07 & 5.23 & 91.57 & 13.53 & 48.47 & 20.02 & 10.2 \\
\hline & U249 & 3.502 & 2.795 & 583.0 & 503.5 & 409.170 & 503.5 & 1.183 & 0.948 & 3.12 & 5.34 & 93.48 & 5.28 & 92.48 & 13.64 & 48.95 & 20.19 & 10.2 \\
\hline & $\mathrm{U} 250$ & 3.501 & 2.792 & 669.5 & 579.1 & 470.600 & 579.3 & 1.366 & 1.089 & 3.11 & 5.33 & 93.28 & 5.30 & 92.80 & 13.50 & 49.29 & 20.25 & 10.2 \\
\hline \multirow{3}{*}{$\begin{array}{l}\mathrm{GB}-4-10 \% \\
\mathrm{Hf} / \mathrm{Ce} / \mathrm{U}\end{array}$} & U228 & 3.500 & 2.779 & 499.3 & 439.6 & 356.870 & 439.6 & 1.051 & 0.835 & 3.01 & 5.32 & 93.09 & 5.29 & 92.73 & 11.96 & 49.90 & 20.60 & 7.5 \\
\hline & U229 & 3.500 & 2.780 & 498.8 & 439.6 & 356.850 & 439.7 & 1.051 & 0.825 & 3.01 & 5.31 & 92.98 & 5.36 & 93.79 & 11.87 & 50.47 & 20.57 & 7.5 \\
\hline & $\mathrm{U} 230$ & 3.500 & 2.783 & 499.5 & 440.2 & 357.340 & \begin{tabular}{|l|}
440.2 \\
\end{tabular} & 1.052 & 0.838 & 3.01 & 5.31 & 93.06 & 5.27 & 92.26 & 11.88 & 49.63 & 20.49 & 7.5 \\
\hline \multirow{4}{*}{\begin{tabular}{|l|} 
Sched 3, 1.5 ksi \\
GB-4-10\% \\
Repeat. GU0705 \\
\end{tabular}} & U268 & 3.500 & 2.747 & 573.5 & 505.5 & 409.270 & 505.7 & 1.267 & 0.994 & 2.87 & 5.24 & 91.81 & 5.23 & 91.68 & 11.86 & 51.66 & 21.51 & 7.5 \\
\hline & U290 & 3.500 & 2.782 & 501.3 & 431.3 & 350.320 & 431.4 & 1.028 & 0.808 & 3.09 & 5.32 & 93.16 & 5.36 & 93.82 & 13.96 & 50.33 & 20.51 & 9.1 \\
\hline & \begin{tabular}{|l}
$\mathrm{U} 291$ \\
\end{tabular} & 3.500 & 2.783 & 502.4 & 431.7 & 350.690 & \begin{tabular}{|l|}
432.0 \\
\end{tabular} & 1.025 & 0.811 & 3.11 & 5.31 & 92.98 & 5.34 & 93.49 & 14.07 & 49.97 & 20.49 & 9.1 \\
\hline & U292 & 3.500 & 2.795 & 508.5 & 436.8 & 354.900 & \begin{tabular}{|l|}
436.9 \\
\end{tabular} & 1.030 & 0.820 & 3.13 & 5.33 & 93.29 & 5.30 & 92.76 & 14.10 & 49.22 & 20.14 & 9.1 \\
\hline \multirow{3}{*}{\begin{tabular}{|l|}
$\mathrm{GB}-4-12 \%$ \\
$\mathrm{Hf} / \mathrm{Ce} / \mathrm{U}$ \\
\end{tabular}} & U269 & 3.494 & 2.782 & 503.4 & 421.9 & 342.120 & 422.9 & 1.012 & 0.809 & 3.17 & 5.23 & 91.53 & 5.23 & 91.67 & 16.18 & 49.31 & 20.38 & 11.1 \\
\hline & $\mathrm{U} 270$ & 3.495 & 2.785 & 498.1 & 417.7 & 338.820 & 418.5 & 1.002 & 0.800 & 3.16 & 5.25 & 91.87 & 5.23 & 91.57 & 16.14 & 49.29 & 20.31 & 11.1 \\
\hline & $\mathrm{U} 271$ & 3.495 & 2.786 & 500.0 & 419.1 & 339.710 & 420.4 & 1.005 & 0.809 & 3. & 5.19 & 90.94 & 5.18 & 90.80 & 16.18 & 48.84 & 20.29 & 11.1 \\
\hline \multirow{6}{*}{\begin{tabular}{|l|}
$3.5 \mathrm{ksi}$ \\
$\mathrm{GB}-5-12 \%$ \\
$\mathrm{Hf} / \mathrm{Ce} / \mathrm{U}$ \\
\end{tabular}} & U272 & 3.495 & 2.799 & 495.0 & 415.1 & 336.670 & \begin{tabular}{|l|l}
415.9 \\
\end{tabular} & 0.993 & 0.798 & 3.17 & 5.24 & 91.70 & 5.16 & 90.31 & 16.15 & 48.45 & 19.91 & 11.1 \\
\hline & U234 & 3.500 & 2.705 & 499.7 & 448.0 & 366.770 & 452.1 & 1.167 & 0.900 & 2.72 & 5.25 & 92.00 & 5.28 & 92.54 & 10.35 & 53.93 & 22.71 & 5.7 \\
\hline & U235 & 3.500 & 2.710 & 499.2 & 447.8 & 362.910 & 447.8 & 1.161 & 0.894 & 2.73 & 5.27 & 92.35 & 5.30 & 92.78 & 10.30 & 53.83 & 22.57 & 5.7 \\
\hline & U236 & 3.500 & 2.710 & 500.4 & 449.0 & 363.760 & 449.0 & 1.163 & 0.902 & 2.73 & 5.27 & 92.22 & 5.26 & 92.20 & 10.27 & 53.49 & 22.57 & 5.7 \\
\hline & $\mathrm{U} 246$ & 3.501 & 2.712 & 549.5 & 494.0 & 400.440 & 494.1 & 1.284 & 1.005 & 2.71 & 5.27 & 92.33 & 5.19 & 90.91 & 10.10 & 53.02 & 22.54 & 5.7 \\
\hline & U247 & 3.500 & 2.719 & 551.4 & 495.6 & 401.730 & 495.8 & 1.281 & 1.007 & 2.73 & 5.27 & 92.27 & 5.17 & 90.56 & 10.12 & 52.55 & 22.31 & 5.7 \\
\hline \multirow[t]{3}{*}{$\begin{array}{l}\mathrm{GB}-6-10 \% \\
\mathrm{Hf} / \mathrm{Ce} / \mathrm{U} \\
\end{array}$} & U240 & 3.500 & N/A & 500.3 & N/A & N/A & N/A & 1.015 & N/A & 3.13 & N/A & N/A & N/A & N/A & N/A & N/A & N/A & 9.2 \\
\hline & U241 & 3.500 & 2.780 & 499.8 & 419.2 & 339.530 & 420.0 & 1.030 & 0.815 & 3.08 & 5.21 & 91.24 & 5.17 & 90.54 & 16.12 & 50.07 & 20.57 & 9.2 \\
\hline & U242 & 3.501 & 2.783 & 499.5 & 418.9 & 339.230 & 419.6 & 1.024 & 0.818 & 3.09 & 5.21 & 91.28 & 5.14 & 89.94 & 16.14 & 49.51 & 20.51 & 9.2 \\
\hline \multirow[t]{2}{*}{$\begin{array}{l}\mathrm{GB}-8-0 \% \\
\mathrm{Hf} / \mathrm{Ce} / \mathrm{U} \\
\end{array}$} & U252 & 3.503 & 2.710 & 506.3 & 475.8 & 387.240 & 475.9 & 1.222 & 0.950 & 2.62 & 5.37 & 93.96 & 5.30 & 92.77 & 6.02 & 53.46 & 22.64 & 0.3 \\
\hline & $\mathrm{U} 253$ & 3.504 & 2.712 & 514.1 & 482.9 & 393.100 & \begin{tabular}{|l}
483.0 \\
\end{tabular} & 1.236 & 0.959 & 2.63 & 5.37 & 94.06 & 5.32 & 93.14 & 6.06 & 53.51 & 22.60 & 0.3 \\
\hline \multirow{3}{*}{$\begin{array}{l}\mathrm{GB}-8-10 \% \\
\mathrm{Hf} / \mathrm{Ce} / \mathrm{U} \\
\end{array}$} & U254 & 3.501 & 2.739 & 499.5 & 434.2 & 353.060 & 434.4 & 1.083 & 0.850 & 2.92 & 5.34 & 93.52 & 5.29 & 92.62 & 13.07 & 51.95 & 21.77 & 8.0 \\
\hline & $\mathrm{U} 255$ & 3.501 & 2.742 & 499.5 & 433.7 & 352.260 & 433.2 & 1.082 & 0.848 & 2.93 & 5.36 & 93.79 & 5.28 & 92.53 & 13.17 & 51.92 & 21.68 & 8.0 \\
\hline & $\mathrm{U} 256$ & 3.502 & 2.738 & 587.4 & 509.1 & 414.070 & 509.2 & 1.271 & 0.995 & 2.93 & 5.35 & 93.71 & 5.30 & 92.85 & 13.33 & 52.14 & 21.82 & 8.0 \\
\hline
\end{tabular}

Page 34 of 49 
Appendix F - Puck Data for Granulation Matrix Testing

\begin{tabular}{|c|c|c|c|c|c|c|c|c|c|c|c|c|c|c|c|c|c|c|}
\hline Batch ID & $\begin{array}{c}\text { Puck } \\
\text { ID }\end{array}$ & $\begin{array}{c}\text { Green } \\
\text { Puck } \\
\text { Diam. } \\
\text { (in.) } \\
\end{array}$ & $\begin{array}{l}\text { Sintered } \\
\text { Puck } \\
\text { Diameter } \\
\text { (in.) } \\
\end{array}$ & $\begin{array}{c}\text { Green } \\
\text { Weight } \\
\text { (g) }\end{array}$ & $\begin{array}{c}\text { Sintered } \\
\text { Puck } \\
\text { Weight } \\
\text { (g) } \\
\end{array}$ & $\begin{array}{c}\text { Puck } \\
\text { Suspended } \\
\text { Weight (g) }\end{array}$ & $\begin{array}{c}\text { Wet } \\
\text { Weight } \\
(\mathrm{g})\end{array}$ & $\begin{array}{c}\text { Green } \\
\text { Puck } \\
\text { Thick. } \\
\text { (in.) } \\
\end{array}$ & \begin{tabular}{|c|} 
Sintered \\
Puck \\
Thick. \\
(in.) \\
\end{tabular} & $\begin{array}{c}\text { Green } \\
\text { Density } \\
\text { (g/cc) }\end{array}$ & $\begin{array}{c}\text { Archi- } \\
\text { medes } \\
\text { Density } \\
\text { (g/cc) } \\
\end{array}$ & $\begin{array}{c}\text { Archi- } \\
\text { medes } \\
\text { Density } \\
\text { (\% TD) }\end{array}$ & $\begin{array}{c}\text { Geom. } \\
\text { Density } \\
\text { (g/cc) }\end{array}$ & $\begin{array}{l}\text { Geom. } \\
\text { (\%TD) }\end{array}$ & $\begin{array}{c}\text { Mass \% } \\
\text { Shrink- } \\
\text { age }\end{array}$ & $\begin{array}{c}\text { Volume } \\
\% \\
\text { Shinkage }\end{array}$ & \begin{tabular}{|c|} 
Diam. \\
$\%$ \\
Shrink- \\
age \\
\end{tabular} & $\begin{array}{l}\text { Powder } \\
\text { \% Moist }\end{array}$ \\
\hline Sched. 3 & U260 & 3.501 & 2.732 & 558.0 & 485.4 & 394.580 & 485.7 & 1.221 & 0.949 & 2.90 & 5.33 & 93.26 & 5.32 & 93.22 & 13.01 & 52.66 & 21.97 & 7.4 \\
\hline & U261 & 3.500 & 2.751 & 549.7 & 477.8 & 387.870 & 478.1 & 1.204 & 0.942 & 2.90 & 5.30 & 92.79 & 5.21 & 91.17 & 13.08 & 51.66 & 21.40 & 7.4 \\
\hline $\begin{array}{l}\text { GB-9-12\% } \\
\mathrm{Hf} / \mathrm{Ce} / \mathrm{U}\end{array}$ & U274 & 3.499 & 2.752 & 500.8 & 422.3 & 342.630 & 423.0 & 1.053 & 0.824 & 3.02 & 5.25 & 91.97 & 5.26 & 92.05 & 15.68 & 51.58 & 21.35 & 9.4 \\
\hline & $\mathrm{U} 275$ & 3.500 & 2.755 & 500.4 & 421.8 & 342.230 & 423.0 & 1.063 & 0.832 & 2.99 & 5.23 & 91.51 & 5.19 & 90.87 & 15.70 & 51.50 & 21.29 & 9.4 \\
\hline & U276 & 3.499 & 2.756 & 499.2 & 421.4 & 341.700 & 422.2 & 1.055 & 0.829 & 3.00 & 5.23 & 91.67 & 5.20 & 91.03 & 15.59 & 51.24 & 21.23 & 9.4 \\
\hline $1.5 \mathrm{ksi}$ & U277 & 3.500 & 2.733 & 498.3 & 420.8 & 341.160 & 421.3 & 1.067 & 0.832 & 2.96 & 5.25 & 91.94 & 5.26 & 92.11 & 15.55 & 52.45 & 21.91 & 9.4 \\
\hline GE-1 & 1939 & 3.253 & 2.634 & 500.5 & 419.7 & 334.503 & 419.7 & 1.202 & 0.967 & 3.06 & 4.93 & 94.74 & 4.86 & 93.44 & 16.14 & 47.24 & 19.03 & 9.6 \\
\hline GD-10 & 2836 & 3.255 & 2.558 & 447.1 & 386.1 & N/A & N/A & 1.180 & 0.927 & 2.78 & N/A & N/A & 4.94 & 95.08 & 13.64 & 51.47 & 21.41 & 7.8 \\
\hline GD-14 & 2837 & 3.254 & 2.553 & 485.8 & 417.9 & 334.754 & 418.0 & 1.298 & 1.017 & 2.75 & 5.02 & 96.54 & 4.90 & 94.17 & 13.98 & 51.76 & 21.54 & 8.6 \\
\hline GD-16 & 2834 & 3.255 & 2.577 & 444.7 & 383.2 & N/A & N/A & 1.164 & 0.920 & 2.80 & $\mathrm{~N} / \mathrm{A}$ & N/A & 4.87 & 93.69 & 13.83 & 50.45 & 20.83 & 7.6 \\
\hline $\begin{array}{l}\text { GD-18 Batch } \\
6 / 12 / 008.5 \text { ton } \\
\end{array}$ & 2532 & 3.506 & 2.729 & 500.7 & 428.2 & 341.949 & 428.2 & 1.160 & 0.907 & 2.73 & 4.96 & 95.47 & 4.92 & 94.69 & 14.48 & 52.62 & 22.16 & 8.5 \\
\hline 6.5 ton & 2533 & 3.506 & 2.725 & 499.5 & 425.3 & 339.419 & 425.4 & 1.164 & 0.906 & 2.71 & 4.95 & 95.12 & 4.91 & 94.43 & 14.85 & 52.97 & 22.28 & 8.5 \\
\hline 7 ton & 2534 & 3.506 & 2.735 & 516.5 & 434.7 & 347.313 & 434.9 & 1.168 & 0.911 & 2.79 & 4.96 & 95.44 & 4.95 & 95.29 & 15.84 & 52.53 & 21.99 & 8.5 \\
\hline 7 ton & 2535 & 3.505 & 2.724 & 517.1 & 432.5 & 345.942 & 432.7 & 1.162 & 0.905 & 2.81 & 4.99 & 95.87 & 5.00 & 96.20 & 16.36 & 52.95 & 22.28 & 8.5 \\
\hline 6.5 ton & 2536 & 3.505 & 2.715 & 510.1 & 426.2 & 340.673 & 426.3 & 1.158 & 0.903 & 2.79 & 4.98 & 95.72 & 4.97 & 95.64 & 16.45 & 53.20 & 22.54 & 8.5 \\
\hline & 2537 & 3.505 & 2.730 & 511.1 & 432.5 & 345.664 & 432.7 & 1.160 & 0.912 & 2.79 & 4.97 & 95.56 & 4.94 & 95.05 & 15.38 & 52.29 & 22.11 & 8.5 \\
\hline 7 ton & 2538 & 3.508 & 2.726 & 518.7 & 434.1 & 347.268 & 434.3 & 1.166 & 0.909 & 2.81 & 4.99 & 95.92 & 4.99 & 95.99 & 16.31 & 52.92 & 22.29 & 8.5 \\
\hline
\end{tabular}

Page 35 of 49 
Figure 1a of Appendix F - Carver Press Archimedes Density Data

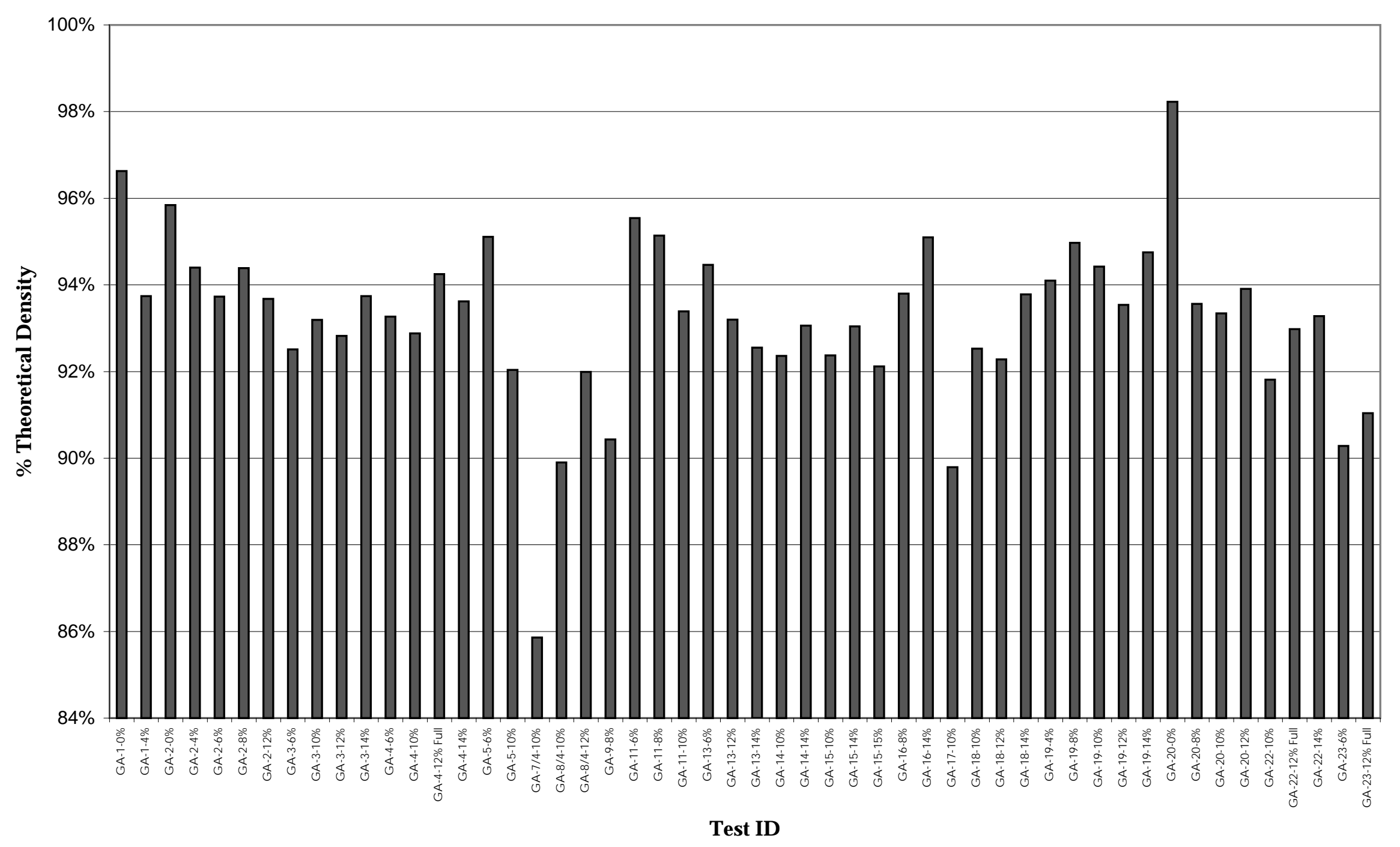


Figure 1b of Appendix F - Carver Press Archimedes Density Data

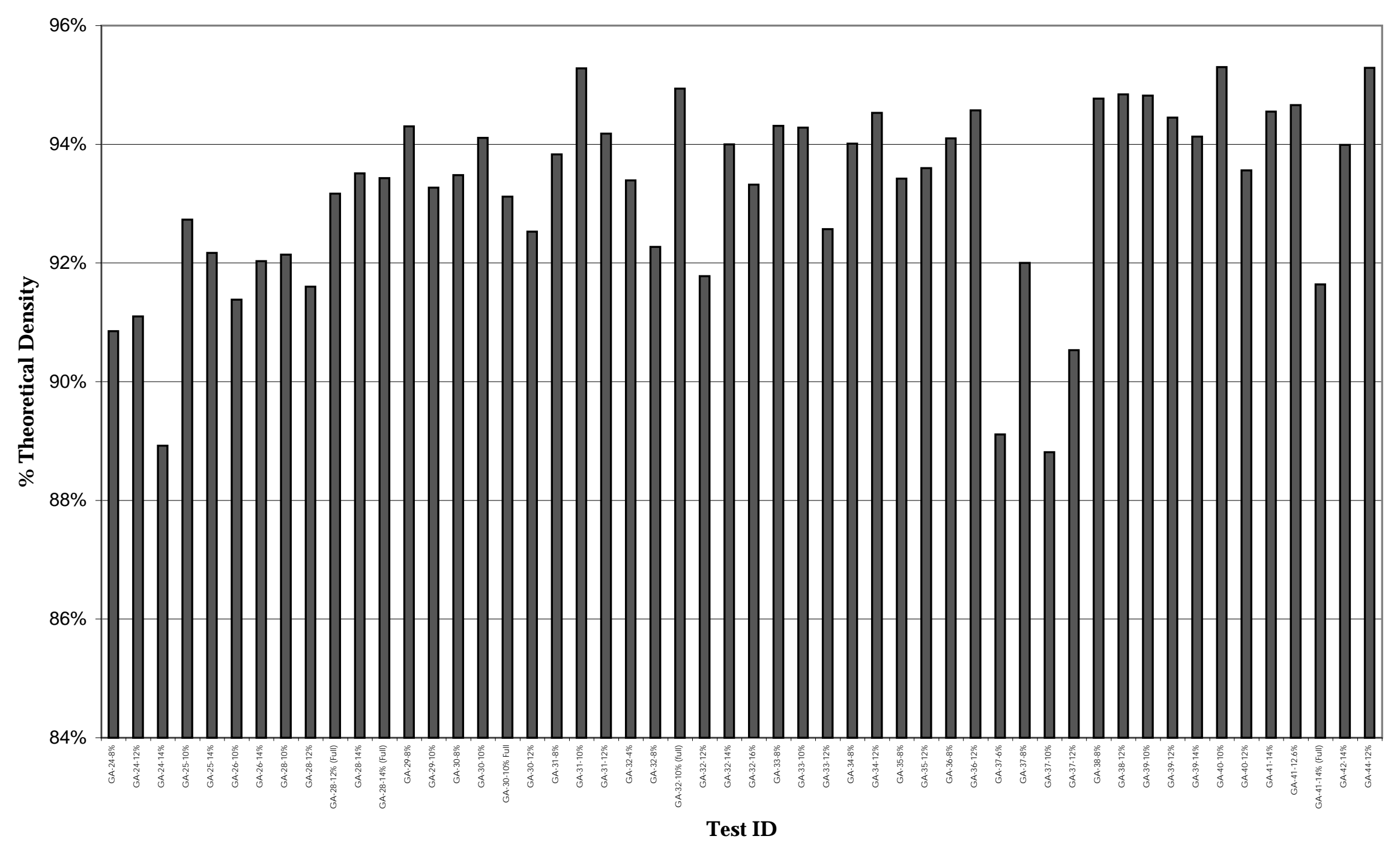


Figure 2 of Appendix F - Best Press Archimedes Density Data

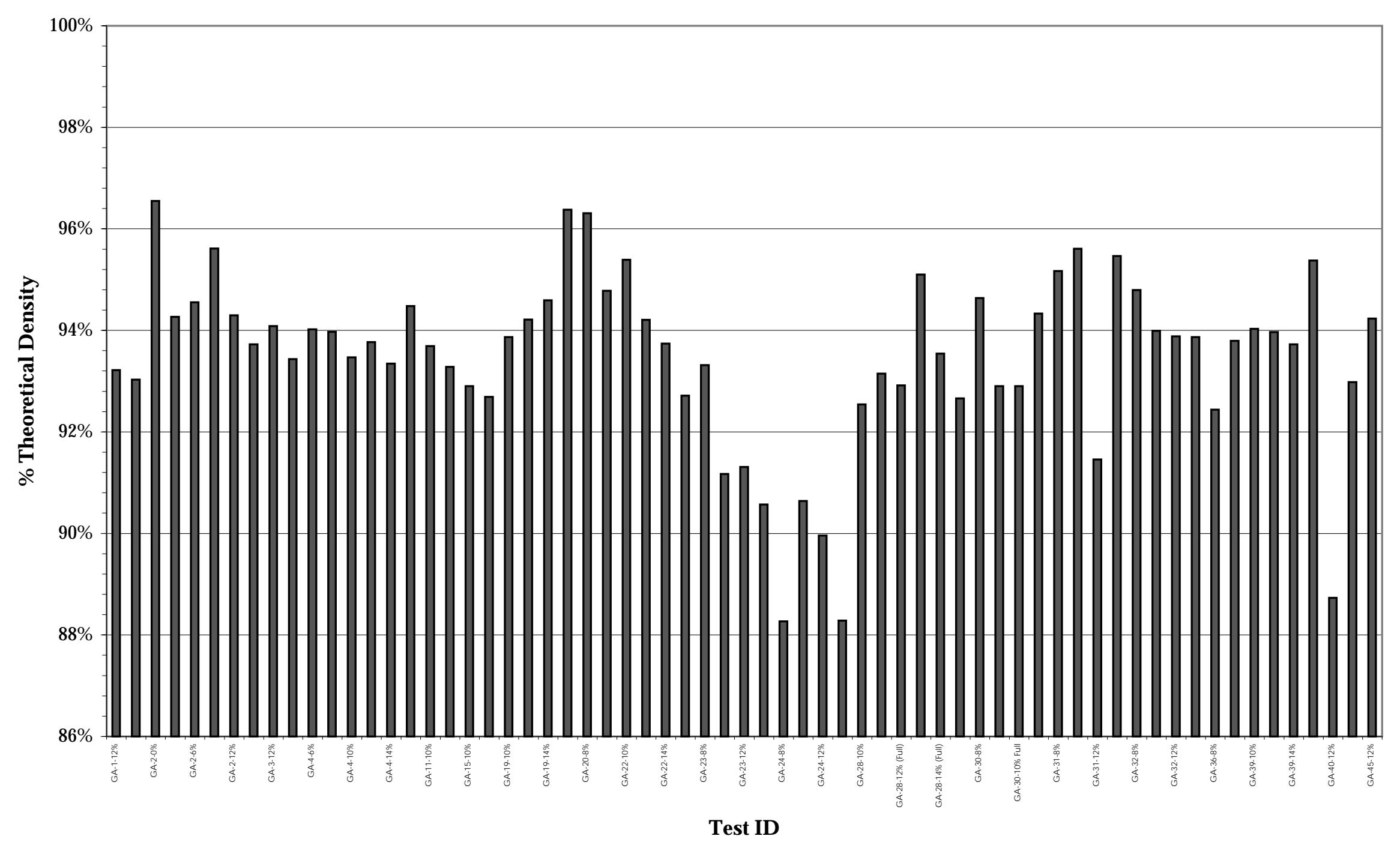


Appendix G - Powder Test Center Data for Granulation Matrix Testing

\begin{tabular}{|c|c|c|c|c|c|c|c|c|c|c|}
\hline Test ID & $\begin{array}{c}\text { Powder } \\
\text { Bulk } \\
\text { Density } \\
(\mathrm{g} / \mathrm{cc}) \\
\end{array}$ & $\begin{array}{c}\text { Powder } \\
\text { Tap } \\
\text { Density } \\
(\mathrm{g} / \mathrm{cc}) \\
\end{array}$ & $\begin{array}{c}\text { Hausner } \\
\text { Ratio }\end{array}$ & $\begin{array}{c}\text { Measured } \\
\text { Angle of } \\
\text { Repose }\end{array}$ & $\begin{array}{c}\text { Compact } \\
\text { Pressure } \\
\text { (psi) }\end{array}$ & $\begin{array}{c}\text { Compact } \\
\text { Slide } \\
\text { Coefficient }\end{array}$ & $\begin{array}{c}\text { Compactability } \\
\text { Coefficient }\end{array}$ & $\begin{array}{l}\text { Ejection } \\
\text { Pressure } \\
\text { (psi) }\end{array}$ & $\begin{array}{c}\text { Axial } \\
\text { Strength } \\
\text { (psi) }\end{array}$ & Cohesiveness \\
\hline \multirow{2}{*}{ GA-1-4\% } & \multirow{2}{*}{1.456} & \multirow{2}{*}{1.627} & \multirow{2}{*}{1.117} & \multirow{2}{*}{74} & 2000 & 0.357 & 0.1544 & 101 & 283 & 0.54 \\
\hline & & & & & 5000 & 0.435 & 0.1565 & 253 & 1430 & 1.30 \\
\hline \multirow{2}{*}{ GA-1-8\% } & \multirow{2}{*}{1.475} & \multirow{2}{*}{1.654} & \multirow{2}{*}{1.121} & \multirow{2}{*}{74} & 2000 & 0.331 & 0.1638 & 77 & 510 & 0.92 \\
\hline & & & & & 5000 & 0.420 & 0.1661 & 203 & 1506 & 1.32 \\
\hline \multirow{2}{*}{ GA-1-12\% } & \multirow{2}{*}{1.555} & \multirow{2}{*}{1.702} & \multirow{2}{*}{1.095} & \multirow{2}{*}{70} & 2000 & 0.324 & 0.1646 & 68 & 180 & 0.31 \\
\hline & & & & & 5000 & 0.400 & 0.1727 & 199 & 1827 & 1.48 \\
\hline \multirow{2}{*}{ GA-2-4\% } & \multirow{2}{*}{1.275} & \multirow{2}{*}{1.482} & & & 2000 & 0.361 & 0.1628 & 103 & 241 & 0.47 \\
\hline & & & 1.162 & 71 & 5000 & 0.444 & 0.1630 & 259 & 2528 & 2.47 \\
\hline CA-2-6\% & 1359 & 1555 & 1144 & 86 & 2000 & 0.332 & 0.1688 & 81 & 412 & 0.79 \\
\hline GA- $-6 \%$ & 1.359 & 1.353 & 1.144 & 80 & 5000 & 0.424 & 0.1709 & 209 & 2935 & 2.73 \\
\hline & & & & & 2000 & 0.338 & 0.1713 & 80 & 430 & 0.79 \\
\hline GA-2-8\% & 1.341 & 1.526 & 1.138 & 75 & 5000 & 0.414 & 0.1744 & 187 & 1314 & 1.18 \\
\hline & & 1577 & & 73 & 2000 & 0.330 & 0.1776 & 96 & 1585 & 3.00 \\
\hline GA-2-12\% & 1.449 & $1.5 \% 7$ & 1.088 & 73 & 5000 & 0.439 & 0.1842 & 280 & 3420 & 3.16 \\
\hline & & & 1108 & 58 & 2000 & 0.519 & 0.1421 & 41 & 620 & 1.93 \\
\hline GA-3-6\% & $1.4 / 6$ & 1.636 & 1.108 & 58 & 5000 & 0.568 & 0.1443 & 117 & 1162 & 1.58 \\
\hline$C A-3-10 \%$ & 1653 & 1769 & 1070 & 59 & 2000 & 0.295 & 0.1417 & 161 & 635 & 1.02 \\
\hline GA-3-10\% & 1.653 & 1.769 & $1.0 / 0$ & 59 & 5000 & 0.367 & 0.1486 & 321 & 1875 & 1.50 \\
\hline & & & & & 2000 & 0.440 & 0.0858 & 115 & 42 & 0.10 \\
\hline GA-3-12\% & 1.650 & 1.769 & $1.0 \% 2$ & 42 & 5000 & 0.376 & 0.1561 & 295 & 2187 & 1.68 \\
\hline & & & & & 2000 & 0.302 & 0.1617 & 94 & 622 & 1.02 \\
\hline GA-3-14\% & 1.656 & 1.779 & 1.074 & 40 & 5000 & 0.400 & 0.1319 & 279 & 1292 & 1.13 \\
\hline GA-4-6\% & 1404 & 1514 & 1078 & 69 & 2000 & 0.364 & 0.1511 & 128 & 687 & 1.35 \\
\hline GA-4-6\% & 1.404 & 1.514 & $1.0 / 8$ & 69 & 5000 & 0.448 & 0.1541 & 251 & 1284 & 1.27 \\
\hline GA-4-8\% & 1517 & 1656 & 1092 & 52 & 2000 & 0.413 & 0.1402 & 28 & 406 & 0.93 \\
\hline GA-4-8\% & 1.517 & 1.656 & 1.092 & 52 & 5000 & 0.468 & 0.1481 & 209 & 1495 & 1.50 \\
\hline$C A-4-10^{\circ}$ & 1401 & 1503 & 1073 & 49 & 2000 & 0.296 & 0.1737 & 123 & 1254 & 2.04 \\
\hline GA-4-10\% & 1.401 & 1.500 & 1.073 & 49 & 5000 & 0.386 & 0.1775 & 243 & 2322 & 1.93 \\
\hline GA-4-10\% & & & & & 2000 & 0.368 & 0.1355 & 100 & 1011 & 2.03 \\
\hline (Full) & 1.498 & 1.616 & 1.079 & 56 & 5000 & 0.434 & 0.1419 & 270 & 1504 & 1.43 \\
\hline$C A-4-12 \%$ & 1535 & 1666 & 1085 & 46 & 2000 & 0.347 & 0.1549 & 86 & 584 & 1.12 \\
\hline GA-4-12\% & 1.535 & 1.666 & 1.085 & 46 & 5000 & 0.424 & 0.1606 & 223 & 1864 & 1.63 \\
\hline CA-4-14\% & 1544 & 1692 & 1096 & 44 & 2000 & 0.325 & 0.1643 & 114 & 1193 & 2.08 \\
\hline GA-4-14\% & 1.544 & 1.692 & 1.096 & 44 & 5000 & 0.412 & 0.1715 & 202 & 1911 & 1.58 \\
\hline$C A-5-6 \%$ & 1317 & 1444 & 1096 & 64 & 2000 & 0.452 & 0.1362 & 75 & 407 & 1.03 \\
\hline GA-5-6\% & 1.317 & 1.444 & 1.096 & 64 & 5000 & 0.540 & 0.1396 & 171 & 1024 & 1.32 \\
\hline GA-5-10\% & 1405 & 1516 & 1079 & 61 & 2000 & 0.383 & 0.1381 & 93 & 368 & 0.75 \\
\hline GA-5-10\% & $1.40 b$ & 1.516 & 1.079 & 61 & 5000 & 0.474 & 0.1442 & 209 & 1429 & 1.53 \\
\hline CA $-7 / 4-10^{\circ}$ & 1129 & 1256 & 1112 & 64 & 2000 & 0.379 & 0.1559 & 83 & 276 & 0.56 \\
\hline GA-7/4-10\% & 1.129 & 1.256 & 1.112 & 64 & 5000 & 0.452 & 0.1605 & 180 & 1287 & 1.30 \\
\hline CA $-8 / 4-10 \%$ & 1055 & 1177 & 1116 & 76 & 2000 & 0.403 & 0.1633 & 71 & 187 & 0.41 \\
\hline GA-8/4-10\% & $1.05 b$ & 1.177 & 1.116 & 76 & 5000 & 0.470 & 0.1657 & 154 & 1013 & 1.07 \\
\hline$C A-8 / 4-12 \%$ & 1430 & 1552 & 1085 & 65 & 2000 & 0.273 & 0.1698 & 164 & 1164 & 1.78 \\
\hline GA-8/4-12\% & 1.430 & 1.552 & 1.085 & 65 & 5000 & 0.374 & 0.1793 & 247 & 3521 & 2.82 \\
\hline GA-11-6\% & 1600 & 1721 & 1076 & 74 & 2000 & 0.440 & 0.1535 & 73 & 172 & 0.43 \\
\hline & 1.600 & 1.721 & 1.076 & 74 & 5000 & 0.512 & 0.1633 & 170 & 2889 & 3.21 \\
\hline GA-11-6\% & 1.600 & 1.721 & 1.076 & 74 & 5000 & 0.529 & 0.1588 & 194 & 855 & 1.07 \\
\hline CA-11-10\% & 1498 & 1594 & 1064 & 70 & 2000 & 0.329 & 0.1980 & 64 & 972 & 1.77 \\
\hline GA-11-10\% & 1.498 & 1.594 & 1.064 & 70 & 5000 & 0.518 & 0.1913 & 149 & 1705 & 1.72 \\
\hline GA-13-6\% & 1210 & & & & 2000 & 0.495 & 0.1812 & 1 & 122 & 0.33 \\
\hline GA-13-6\% & 1.210 & 1.427 & 1.179 & 72 & 5000 & 0.556 & 0.1773 & 127 & 1597 & 2.05 \\
\hline
\end{tabular}


Appendix G - Powder Test Center Data for Granulation Matrix Testing

\begin{tabular}{|c|c|c|c|c|c|c|c|c|c|c|}
\hline Test ID & $\begin{array}{c}\text { Powder } \\
\text { Bulk } \\
\text { Density } \\
(\mathrm{g} / \mathrm{cc}) \\
\end{array}$ & $\begin{array}{c}\text { Powder } \\
\text { Tap } \\
\text { Density } \\
(\mathrm{g} / \mathrm{cc}) \\
\end{array}$ & $\begin{array}{c}\text { Hausner } \\
\text { Ratio }\end{array}$ & $\begin{array}{c}\text { Measured } \\
\text { Angle of } \\
\text { Repose }\end{array}$ & $\begin{array}{c}\text { Compact } \\
\text { Pressure } \\
\text { (psi) }\end{array}$ & $\begin{array}{c}\text { Compact } \\
\text { Slide } \\
\text { Coefficient }\end{array}$ & $\begin{array}{l}\text { Compactability } \\
\text { Coefficient }\end{array}$ & $\begin{array}{l}\text { Ejection } \\
\text { Pressure } \\
\text { (psi) }\end{array}$ & \begin{tabular}{|c|} 
Axial \\
Strength \\
(psi)
\end{tabular} & Cohesiveness \\
\hline \multirow{2}{*}{ GA-13-12\% } & \multirow{2}{*}{1.367} & \multirow{2}{*}{1.464} & \multirow{2}{*}{1.071} & \multirow{2}{*}{63} & 2000 & 0.294 & 0.2232 & 103 & 894 & 1.45 \\
\hline & & & & & 5000 & 0.495 & 0.2050 & 174 & 1850 & 1.79 \\
\hline \multirow{2}{*}{ GA-13-14\% } & \multirow{2}{*}{1.565} & \multirow{2}{*}{1.668} & \multirow{2}{*}{1.066} & \multirow{2}{*}{56} & 2000 & 0.302 & 0.1897 & 124 & 1411 & 2.29 \\
\hline & & & & & 5000 & 0.493 & 0.1864 & 206 & 2636 & 2.62 \\
\hline \multirow{2}{*}{ GA-15-10\% } & \multirow{2}{*}{1.567} & \multirow{2}{*}{1.668} & \multirow{2}{*}{1.064} & \multirow{2}{*}{67} & 2000 & 0.336 & 0.1376 & 169 & 814 & 1.56 \\
\hline & & & & & 5000 & 0.361 & 0.1430 & 358 & 1228 & 0.96 \\
\hline \multirow{2}{*}{ GA-15-14\% } & \multirow{2}{*}{1.478} & \multirow{2}{*}{1.594} & & & 2000 & 0.299 & 0.1768 & 28 & 1213 & 2.02 \\
\hline & & & 1.078 & 58 & 5000 & 0.358 & 0.1795 & 334 & 1891 & 1.35 \\
\hline$C A-15-15 \%$ & 1525 & 1663 & 1090 & 50 & 2000 & 0.279 & 0.1723 & 153 & 696 & 1.07 \\
\hline GA-15-15\% & $1.5 \angle 5$ & 1.663 & 1.090 & 50 & 5000 & 0.370 & 0.1735 & 309 & 3269 & 2.41 \\
\hline & & & & & 2000 & 0.489 & 0.1312 & 25 & 242 & 0.67 \\
\hline GA-19-4\% & 1.389 & $1.55 \%$ & 1.121 & 65 & 5000 & 0.543 & 0.1329 & 129 & 1007 & 1.32 \\
\hline GA-19-8\% & 1505 & 1674 & 1112 & 65 & 2000 & 0.315 & 0.1471 & 102 & 346 & 0.60 \\
\hline GA-19-8\% & 1.505 & $1.6 / 4$ & 1.112 & 65 & 5000 & 0.381 & 0.1502 & 265 & 1245 & 1.02 \\
\hline & & & & & 2000 & 0.308 & 0.1409 & 63 & 1140 & 1.96 \\
\hline GA-19-10\% & 1.724 & $1.8 / 2$ & 1.086 & 64 & 5000 & 0.362 & 0.1499 & 287 & 1633 & 1.26 \\
\hline GA-19-10\% & 1487 & 1629 & 1095 & 60 & 2000 & 0.390 & 0.1717 & 119 & 3622 & 2.86 \\
\hline Repeat & $1.48 /$ & 1.629 & 1.095 & 60 & 5000 & 0.290 & 0.1687 & 154 & 1227 & 1.91 \\
\hline GA-19-12\% & 1.587 & 1.705 & 1.074 & 41 & 2000 & 0.274 & 0.1534 & 167 & 601 & 0.93 \\
\hline GA-19-14\% & & & 1.072 & 40 & 2000 & 0.271 & 0.1772 & 208 & 1249 & 1.90 \\
\hline GA-19-14\% & 1.560 & $1.6 / 3$ & 1.072 & 40 & 5000 & 0.366 & 0.1767 & 323 & 2729 & 1.94 \\
\hline GA-20-8\% & 1412 & 1556 & 1102 & 58 & 2000 & 0.293 & 0.1604 & 163 & 784 & 1.26 \\
\hline & 1.412 & 1.356 & & 30 & 5000 & 0.348 & 0.1656 & 330 & 1985 & 1.47 \\
\hline & & & & & 2000 & 0.280 & 0.1893 & 166 & 1251 & 1.95 \\
\hline GA-2U-10\% & 1.204 & 1.260 & 1.047 & 62 & 5000 & 0.339 & 0.1940 & 322 & 3688 & 2.73 \\
\hline & & & & & 2000 & 0.299 & 0.1708 & 156 & 589 & 0.96 \\
\hline GA-2U-12\% & 1.421 & 1.493 & 1.051 & 69 & 5000 & 0.340 & 0.1775 & 351 & 3494 & 2.51 \\
\hline & & & & & 2000 & 0.315 & 0.1501 & 155 & 610 & 1.05 \\
\hline GA-22-10\% & 1.313 & 1.451 & $1.10 b$ & 54 & 5000 & 0.387 & 0.1466 & 278 & 2265 & 1.90 \\
\hline CA-22-12\% & 1407 & 1539 & 1094 & 52 & 2000 & 0.287 & 0.1453 & 119 & 705 & 1.09 \\
\hline$G A-\angle 2-1 \angle \%$ & 1.407 & 1.539 & 1.094 & 52 & 5000 & 0.361 & 0.1441 & 258 & 1235 & 0.92 \\
\hline & & & & & 2000 & 0.314 & 0.1550 & 107 & 1060 & 1.85 \\
\hline GA-22-14\% & 1.475 & 1.565 & 1.061 & 50 & 5000 & 0.384 & 0.1594 & 359 & 1675 & 1.37 \\
\hline & & & & & 2000 & 0.461 & 0.1441 & 154 & 493 & 1.28 \\
\hline GA-23-6\% & 1.324 & 1.514 & 1.144 & 71 & 5000 & 0.581 & 0.1495 & 265 & 1297 & 1.92 \\
\hline & & & 1107 & & 2000 & 0.461 & 0.1441 & 117 & 706 & 1.82 \\
\hline GA-23-8\% & 1.410 & 1.561 & 1.107 & 65 & 5000 & 0.557 & 0.1512 & 218 & 1515 & 2.07 \\
\hline CA-23-10\% & 1243 & 1373 & 1105 & 73 & 2000 & 0.332 & 0.1540 & 148 & 239 & 0.00 \\
\hline GA- $23-10 \%$ & 1.243 & $1.3 / 3$ & 1.100 & 73 & 5000 & 0.409 & 0.1519 & 375 & 2275 & 1.96 \\
\hline CA-23-12\% & 1453 & 1534 & 1056 & 53 & 2000 & 0.315 & 0.1444 & 128 & 874 & 1.59 \\
\hline GA-23-12\% & 1.453 & 1.534 & 1.056 & 53 & 5000 & 0.409 & 0.1478 & 355 & 2984 & 2.54 \\
\hline GA-24-8\% & 1082 & 1236 & 1142 & 74 & 2000 & 0.325 & 0.1707 & 217 & 471 & 0.83 \\
\hline GA-24-8\% & 1.082 & 1.236 & 1.142 & 74 & 5000 & 0.410 & 0.1682 & 363 & 2035 & 1.79 \\
\hline GA-24-12\% & 1315 & 1440 & 1095 & 52 & 2000 & 0.297 & 0.1531 & 96 & 348 & 0.70 \\
\hline$G A-24-12 \%$ & 1.315 & 1.440 & 1.095 & 52 & 5000 & 0.386 & 0.1435 & 407 & 1977 & 1.66 \\
\hline CA-24-14\% & 1391 & 1474 & 1060 & 57 & 2000 & 0.333 & 0.1479 & 108 & 752 & 1.35 \\
\hline GA- $\angle 4-14 \%$ & 1.391 & $1.4 / 4$ & 1.060 & $5 /$ & 5000 & 0.396 & 0.1577 & 317 & 2637 & 2.25 \\
\hline GA-26-14\% & 1629 & 1733 & 1064 & 55 & 2000 & 0.336 & 0.1718 & 79 & 458 & 0.83 \\
\hline GA-2b-14\% & 1.629 & 1.733 & 1.064 & 55 & 5000 & 0.472 & 0.1751 & 208 & 1356 & 1.36 \\
\hline GA-26-14\% & 1518 & & 1095 & & 2000 & 0.317 & 0.1580 & 148 & 419 & 0.74 \\
\hline Repeat & 1.518 & 1.662 & 1.095 & 51 & 5000 & 0.425 & 0.1667 & 312 & 1474 & 1.32 \\
\hline
\end{tabular}


Appendix G - Powder Test Center Data for Granulation Matrix Testing

\begin{tabular}{|c|c|c|c|c|c|c|c|c|c|c|}
\hline Test ID & $\begin{array}{l}\text { Powder } \\
\text { Bulk } \\
\text { Density } \\
\text { (g/cc) } \\
\end{array}$ & $\begin{array}{l}\text { Powder } \\
\text { Tap } \\
\text { Density } \\
\text { (g/cc) } \\
\end{array}$ & $\begin{array}{c}\text { Hausner } \\
\text { Ratio }\end{array}$ & $\begin{array}{c}\text { Measured } \\
\text { Angle of } \\
\text { Repose }\end{array}$ & $\begin{array}{c}\text { Compact } \\
\text { Pressure } \\
\text { (psi) }\end{array}$ & $\begin{array}{l}\text { Compact } \\
\text { Slide } \\
\text { Coefficient }\end{array}$ & $\begin{array}{c}\text { Compactability } \\
\text { Coefficient }\end{array}$ & $\begin{array}{l}\text { Ejection } \\
\text { Pressure } \\
\text { (psi) }\end{array}$ & $\begin{array}{c}\text { Axial } \\
\text { Strength } \\
\text { (psi) }\end{array}$ & Cohesiveness \\
\hline \multirow{2}{*}{ GA-28-10\% } & \multirow{2}{*}{1.227} & \multirow{2}{*}{1.389} & \multirow{2}{*}{1.132} & \multirow{2}{*}{76} & 2000 & 0.337 & 0.1825 & 110 & 677 & 1.28 \\
\hline & & & & & 5000 & 0.426 & 0.1824 & 269 & 2474 & 2.22 \\
\hline \multirow{2}{*}{ GA-28-12\% } & \multirow{2}{*}{1.190} & \multirow{2}{*}{1.320} & \multirow{2}{*}{1.109} & \multirow{2}{*}{70} & 2000 & 0.384 & 0.1725 & 133 & 571 & 1.32 \\
\hline & & & & & 5000 & 0.480 & 0.1739 & 273 & 1001 & 1.09 \\
\hline \multirow{2}{*}{$\begin{array}{c}\text { GA-28-12\% } \\
\text { Full }\end{array}$} & \multirow{2}{*}{1.239} & \multirow{2}{*}{1.363} & \multirow{2}{*}{1.100} & \multirow{2}{*}{71} & 2000 & 0.341 & 0.1683 & 118 & 698 & 1.36 \\
\hline & & & & & 5000 & 0.410 & 0.1693 & 373 & 1044 & 0.94 \\
\hline \multirow{2}{*}{ GA-28-14\% } & \multirow{2}{*}{1.472} & \multirow{2}{*}{1.577} & \multirow{2}{*}{1.071} & \multirow{2}{*}{59} & 2000 & 0.326 & 0.1648 & 100 & 853 & 1.52 \\
\hline & & & & & 5000 & 0.424 & 0.1673 & 259 & 1135 & 1.00 \\
\hline \multirow{2}{*}{$\begin{array}{c}\text { GA-28-14\% } \\
\text { Full } \\
\end{array}$} & \multirow{2}{*}{1.381} & \multirow{2}{*}{1.440} & \multirow{2}{*}{1.043} & \multirow{2}{*}{70} & 2000 & 0.305 & 0.1717 & 161 & 327 & 0.56 \\
\hline & & & & & 5000 & 0.400 & 0.1750 & 348 & 1175 & 1.02 \\
\hline
\end{tabular}


Figure 1 of Appendix G - Measured Powder Densities

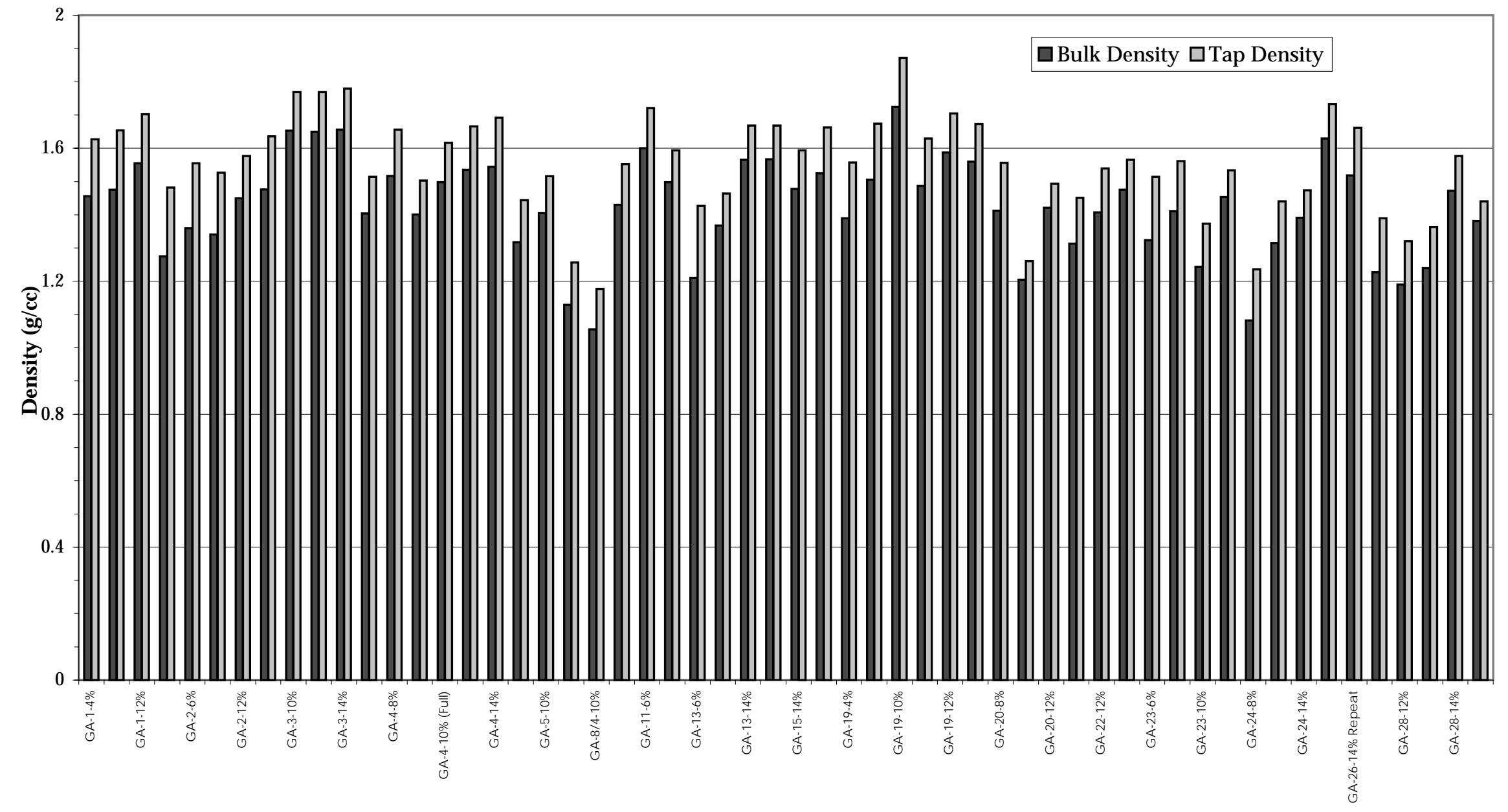


Figure 2 of Appendix G - Hausner Ratio

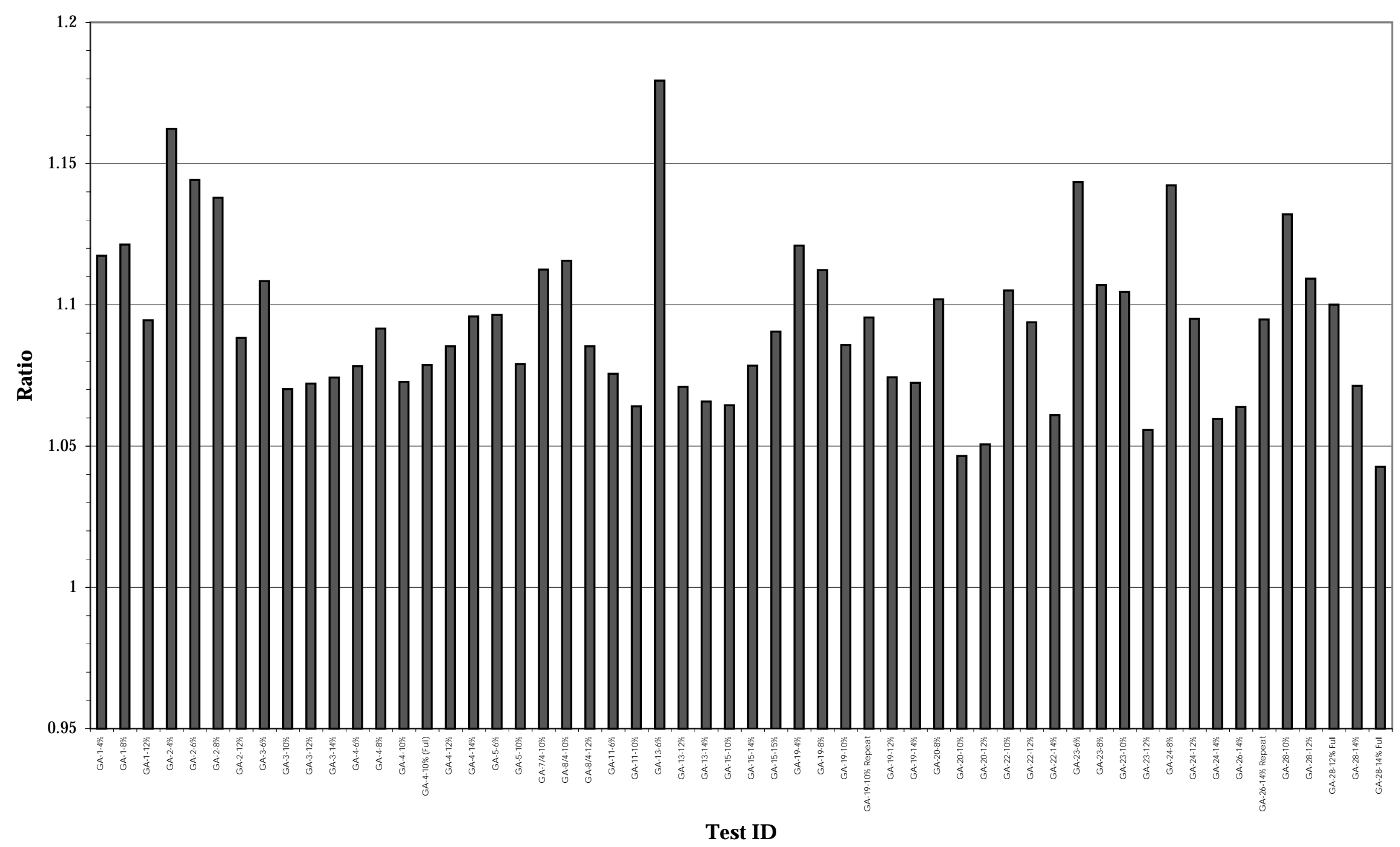


Figure 3 of Appendix G - Angle of Repose as Measured by the PTC

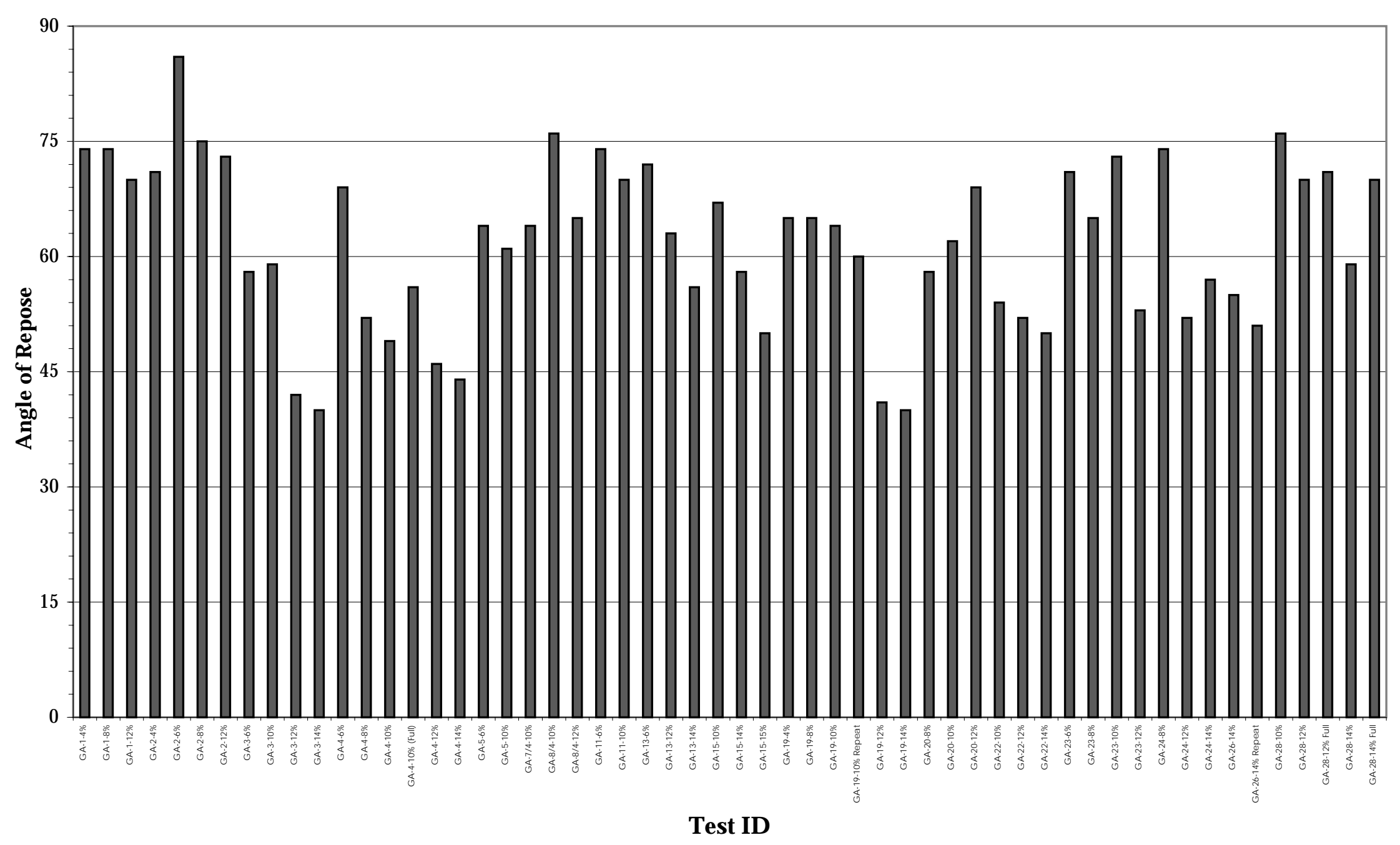


Figure 4 of Appendix G - Slide Coefficients as Measured by the PTC

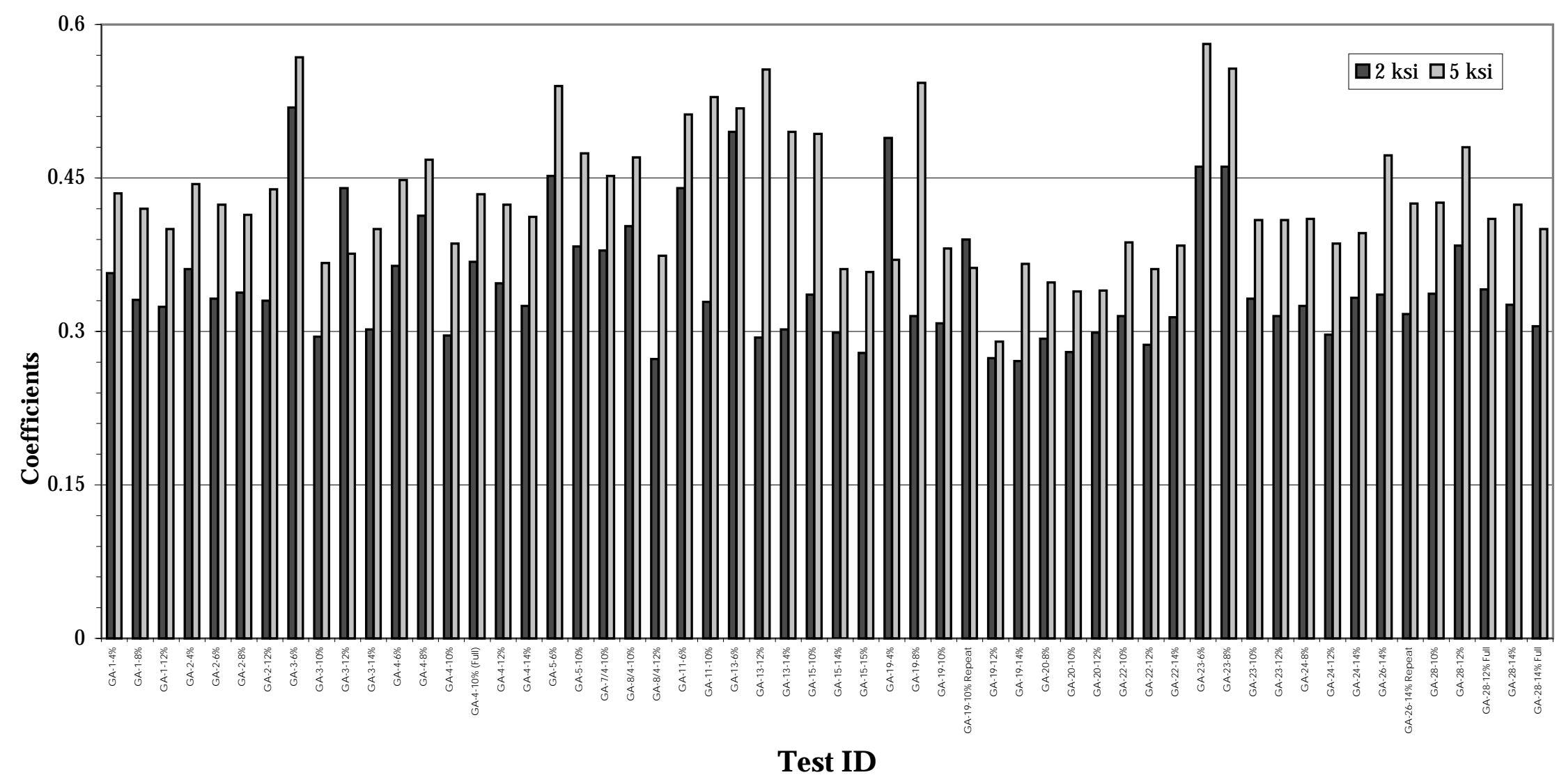


Figure 5 of Appendix G - Compactability Coefficients as Measured by the PTC

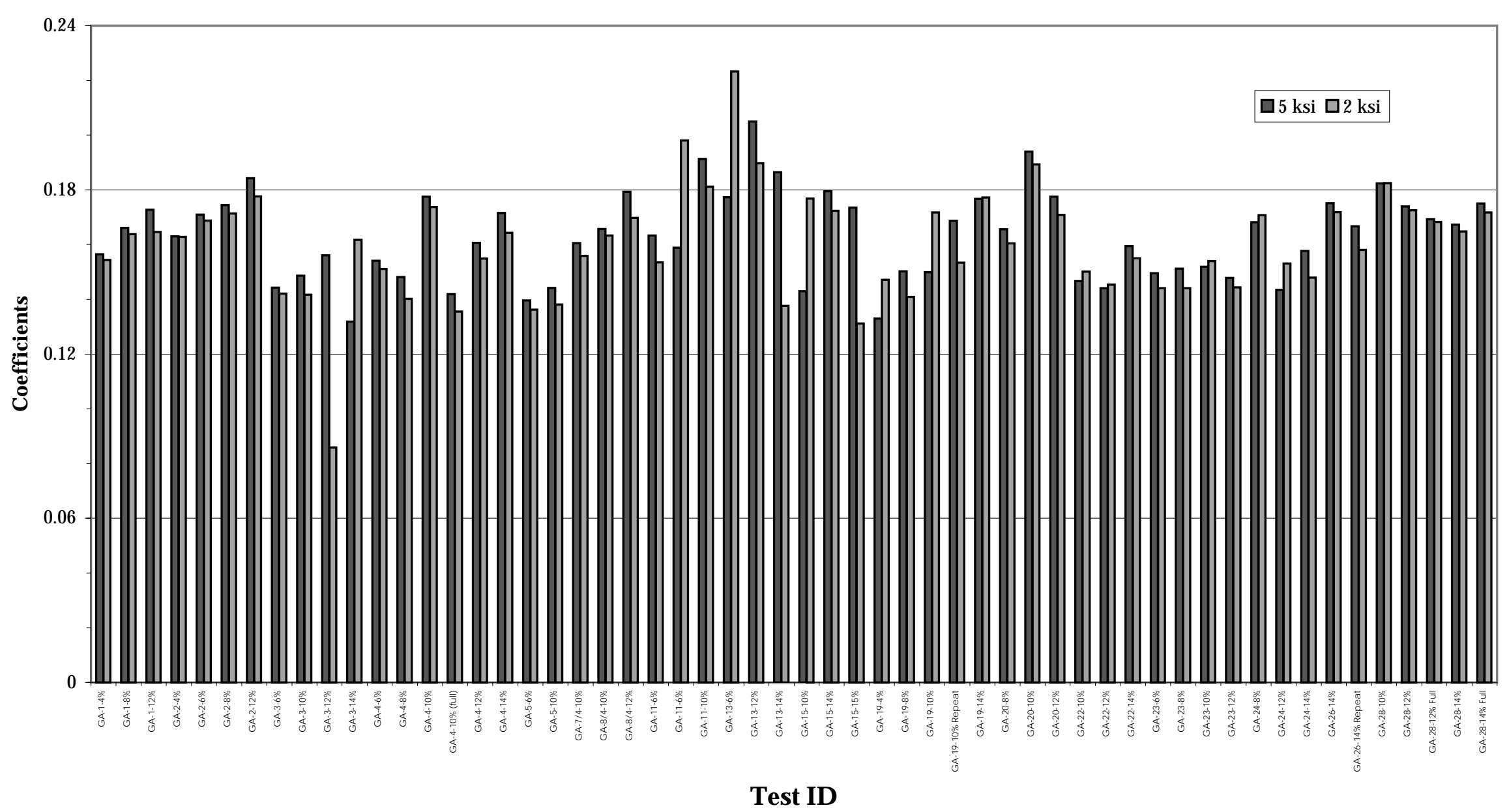


Figure 6 of Appendix G - Ejection Pressure as Measured by the PTC

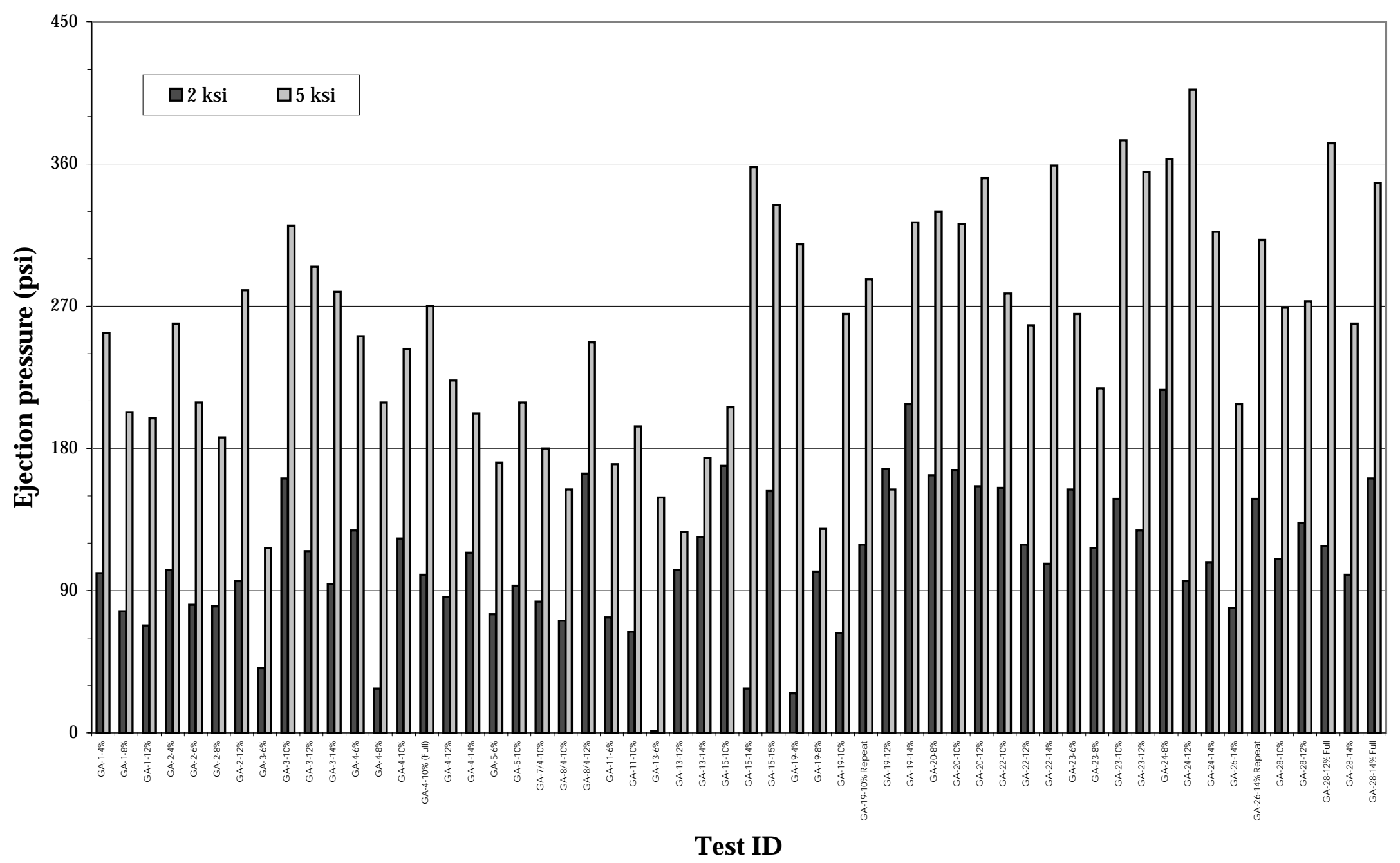


Figure 7 of Appendix G - Axial Strength as Measured by the PTC

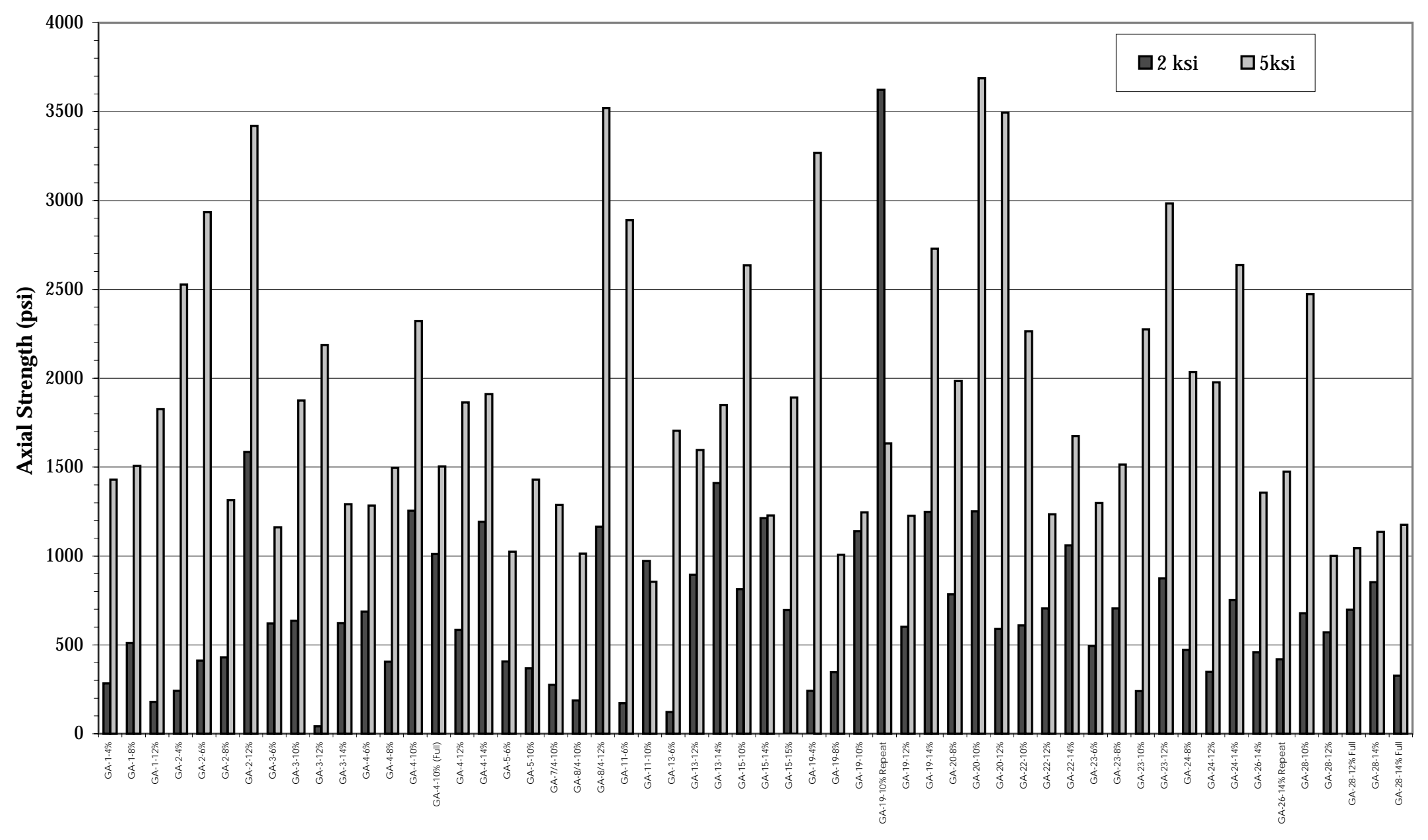

Test ID 
Figure 8 of Appendix G - Cohesiveness as Measured by the PTC

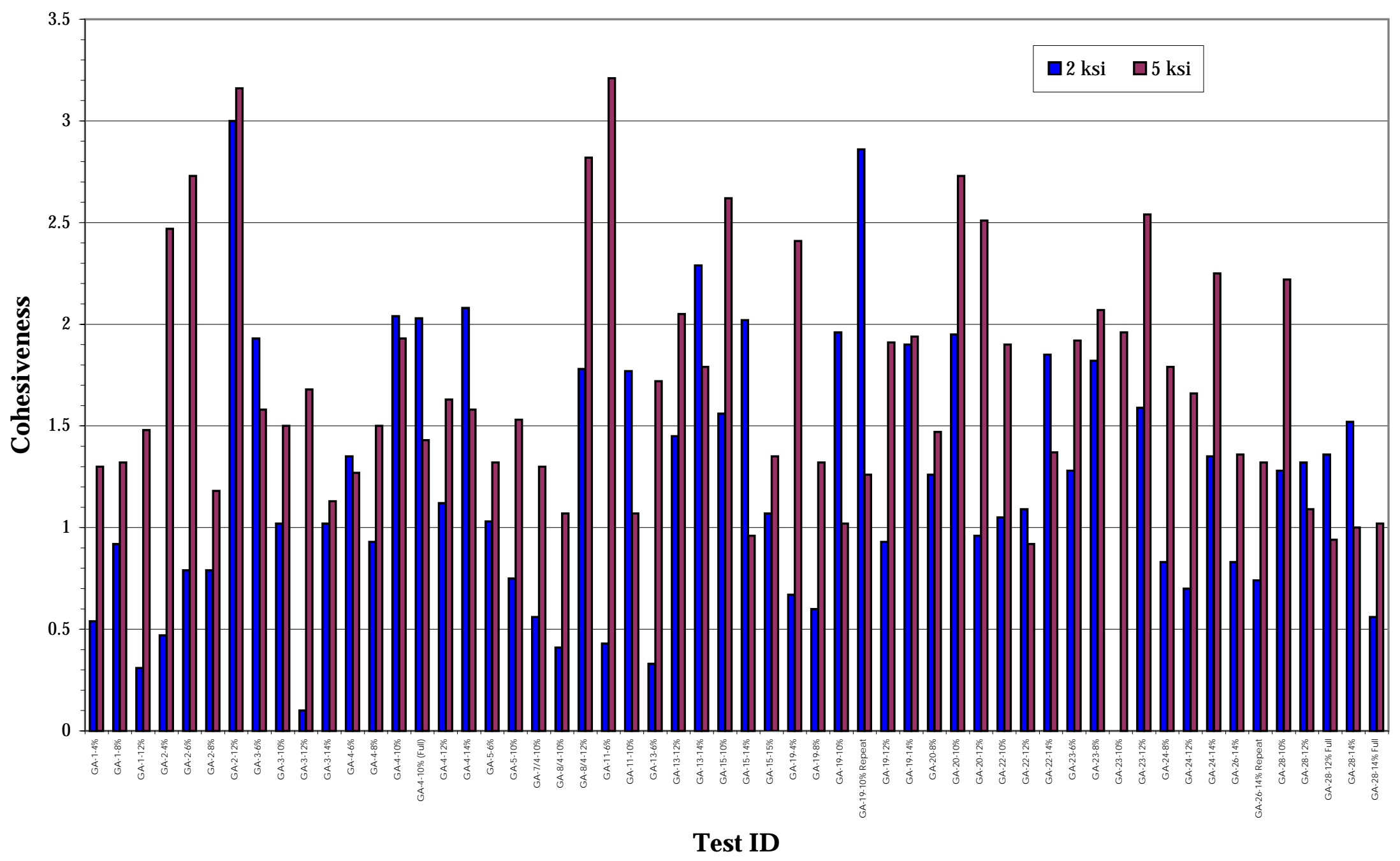

